\title{
NUMERICAL STUDY OF PLASMA-ASSISTED AERODYNAMIC CONTROL FOR HYPERSONIC VEHICLES
}

\author{
by \\ Nicholas J. Bisek \\ A dissertation submitted in partial fulfillment \\ of the requirements for the degree of \\ Doctor of Philosophy \\ (Aerospace Engineering) \\ in The University of Michigan \\ 2010
}

Doctoral Committee:

Professor Iain D. Boyd, Chair

Professor Mark J. Kushner

Professor Kenneth G. Powell

Jonathan Poggie, U.S. Air Force Research Laboratory 
The only a question we have in life, is what we do with the time we have. Gandelf, The Lord of the Rings: The Fellowship of the Ring 
C) Nicholas J. Bisek

2010

All Rights Reserved 
To my mother. 


\section{ACKNOWLEDGEMENTS}

I want to start by thanking my dissertation committee for their patience, support and guidance. Their willingness to help and insightful suggestions have made this journey easier. I especially want to thank Professor Iain Boyd for agreeing to be

my advisor. He has been a great mentor who has not only given me the freedom to explore nonessential aspects of my research, but the confidence to do so. Without that freedom, I doubt I would have had the opportunity to work so closely with Dr. Jonathan Poggie, whose expertise and reputation in the field of computational hypersonics has enabled him to provide me with additional guidance and insight over the last few years. To a large extent, Dr. Poggie has become my second advisor, and while some may think that having two advisers would be burden (i.e., two bosses, differing opinions, etc.), the experience and opportunities it has provided have been invaluable. My relationship with both Professor Boyd and Dr. Poggie has given me the chance to peer into both academic and professional career paths and provided insight that will greatly influence my own future career.

Although my research with Professor Mark Kushner started a little outside the scope of my thesis and its original goals, I am grateful that we decided to risk the detour. His eagerness and active participation created a unique opportunity for my research to cross disciplines which has provided generality and increased applicability of the methods developed. I would also like to thank Professor Ken Powell, who has always been available to discuss research or any other situations occurring in my life. 
Family has, and always will be, a big part of my life. They provide unquestionable comfort and support, while inspiring me to reach my full potential. They have also been eager to hear my progress and even 'attempt' to read my publications, and for that I thank them.

I want to thank all my friends, both those I have met while at the University of Michigan, and those who came into my life prior to graduate studies. They have always been supportive and great listeners, especially during the hardships. I would like to especially thank Erin Farbar, Jinhui Zhao, Andy Klesh, Eric Gustafson, Amor Menezes, Pat Trizilia, Tim Deshchenes, Alexandre Martin, Jon Rabe, and Sam Jens. As is customary with acknowledgments, the most important person comes last. I want to thank my fiancé Jamie. She has been closer to me this last year and a half than anyone. I think the road would have been much more difficult without her. Her patience, understanding personality, and love have been instrumental to my success and I hope they will continue to be my inspiration as we strive toward our dreams. 


\section{TABLE OF CONTENTS}

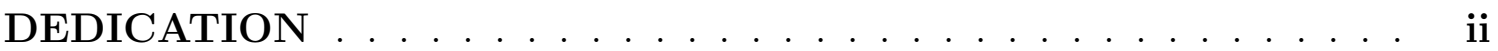

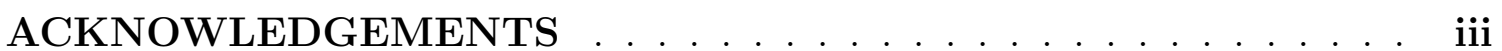

LIST OF FIGURES . . . . . . . . . . . . . . . . . viii

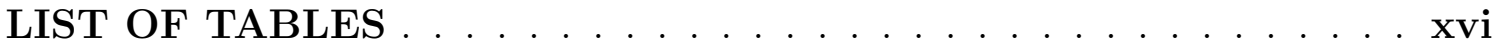

LIST OF APPENDICES . . . . . . . . . . . . . . . xix

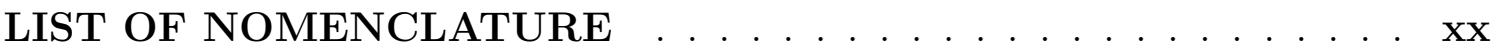

\section{CHAPTER}

I. Introduction $\ldots \ldots \ldots \ldots \ldots \ldots$

1.1 Review of Related Work . . . . . . . . . . . . . . . . . . . 4

1.2 Thesis Overview . . . . . . . . . . . . . . . . . 10

II. Navier-Stokes Solver _ . . . . . . . . . . . . . . . . . . . . . . . . 14

2.1 LeMANS: An Overview . . . . . . . . . . . . . . . . . . . 15

2.2 LeMANS: Validation . . . . . . . . . . . . . . . . . . . . . 18

2.2.1 Sharp-Nose Elliptic Cone . . . . . . . . . . . . 19

2.2 .2 Blunt-Nose Elliptic Cone . . . . . . . . . . . 25

2.3 LeMANS: New Features . . . . . . . . . . . . . . . . . . . . 33

2.3.1 Pre-LeMANS . . . . . . . . . . . . . . . . 33

2.3 .2 Post-LeMANS . . . . . . . . . . . . . . . 34

2.3.3 Phenomenological Model of Volumetric Heating . . 35

2.3 .4 LeMANS-MHD . . . . . . . . . . . . . . . . . 35

2.3.5 Electrical Conductivity Models . . . . . . . . . 36

2.4 Summary . . . . . . . . . . . . . . . . . . 37

III. Phenomenological Model of Volumetric Heating . . . . . . . 38

3.1 Introduction . . . . . . . . . . . . . . . . 38

3.2 Phenomenological Heating Model . . . . . . . . . . . . . . 38 
3.3 Validation . . . . . . . . . . . . . . . . . . 40

3.4 Energy Deposition Results . . . . . . . . . . . . . . . . 43

3.4.1 Reference Geometry . . . . . . . . . . . . . . . . 44

3.4 .2 Reference Pitching Moment . . . . . . . . . . . . 46

3.4.3 Study Parameters . . . . . . . . . . . . . . . . . 49

3.4.4 Parametric Study Results . . . . . . . . . . . . . 52

3.4.5 Hot Wall Effect . . . . . . . . . . . . . . . 55

3.5 Additional Vehicle Configurations . . . . . . . . . . . 57

3.6 Conclusions . . . . . . . . . . . . . . . . . . . . 64

IV. Magnetohydrodynamics . . . . . . . . . . . 66

4.1 Introduction . . . . . . . . . . . . . . 66

4.2 Magnetohydrodynamic Model . . . . . . . . . . . . . . 66

4.2.1 Low Magnetic Reynolds Number Approximation . . 69

4.2 .2 Implementation . . . . . . . . . . . . . . . . 71

4.2.3 MHD Boundary Conditions . . . . . . . . . . . . 74

4.3 Validation of the MHD Solver . . . . . . . . . . . . . 75

4.3.1 Analytical case with Dirichlet Boundary Conditions 76

4.3.2 Flow Between Two Electrodes . . . . . . . . . . . . 79

4.4 Parallel MHD Solver . . . . . . . . . . . . . . . . . . . . . . 81

4.4.1 Flow Between Parallel Electrodes . . . . . . . . . 83

4.4 .2 Parallel Efficiency . . . . . . . . . . . . . . . . . 84

4.5 Hall Effect . . . . . . . . . . . . . . . . . . . . . . 88

4.5.1 Segmented Electrodes . . . . . . . . . . . . 89

4.6 Conclusions . . . . . . . . . . . . . . . . . . 95

V. Electrical Conductivity Modeling . . . . . . . . . . 96

5.1 Boltzmann Solver . . . . . . . . . . . . . . . . . . . . 97

5.2 Electrical Conductivity of Air . . . . . . . . . . . . . . 101

5.2 .1 Semi-Analytic Models . . . . . . . . . . . . . . 102

5.2 .2 Comparisons . . . . . . . . . . . . . . . . . 105

5.3 Surrogate Modeling . . . . . . . . . . . . . . . . 110

5.3 .1 Surrogates . . . . . . . . . . . . . . . . . 110

5.3 .2 Dimensions . . . . . . . . . . . . . . . . . . . . . . . 113

5.3.3 Reduced Dimensional Modeling . . . . . . . . . 115

5.3 .4 Conservativeness. . . . . . . . . . . . . . . . . . 124

5.3.5 Global Sensitivity Analysis . . . . . . . . . . 126

5.3.6 Computational Cost Analysis . . . . . . . . . . . . . 128

5.3.7 Summary of Argon Models . . . . . . . . . . . . 130

5.4 Surrogate Models of Air . . . . . . . . . . . . . . . . 130

5.4 .1 Constricted Design Space . . . . . . . . . . . . . 133

5.4 .2 Summary of Air Models . . . . . . . . . . . . . 137 
5.5 Surrogate Models - Applied . . . . . . . . . . . . . . . 138

5.6 Conclusions . . . . . . . . . . . . . . . . . . . . . . . 142

VI. Computational Analysis of MHD Applications . . . . . . . 144

6.1 Arc Discharge . . . . . . . . . . . . . . . . . . . 144

6.2 MHD-Heat Shield . . . . . . . . . . . . . . . . . . . . . . 149

6.3 Conclusions . . . . . . . . . . . . . . . . . . . 163

VII. Conclusions . . . . . . . . . . . . . . . . . . 165

7.1 Summary . . . . . . . . . . . . . . . . . 165

7.2 Contributions . . . . . . . . . . . . . . 170

7.2 .1 Major Contributions . . . . . . . . . . . 170

7.2 .2 Improvements to LeMANS . . . . . . . . . . . 172

7.3 Future Work . . . . . . . . . . . . . . . . . . 174

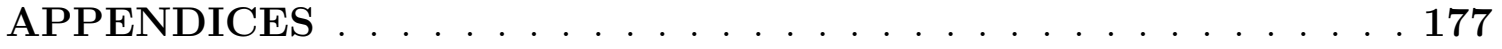

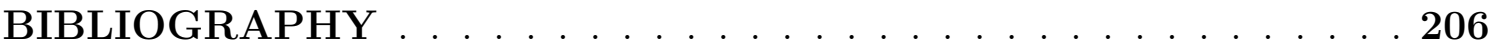




\section{LIST OF FIGURES}

\section{Figure}

1.1 The 'near-space' region of the atmosphere. Images from left to right: US Air Force photos (http://www.af.mil, image: 080204-F-1001W030), NASA Dryden photo collection (http://www.dfrc.nasa.gov, image: ED99-45243-01), NASA's image of the day (http://www.nasa.gov,

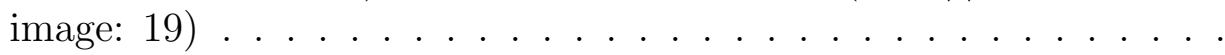

1.2 Mach 5.5 wave rider being prepared for a full scale wind tunnel test. (Great Images in NASA library, http://grin.hq.nasa.gov, image: GPN-2000-001927) . . . . . . . . . . . . . . .

2.1 Surface of the sharp-nose elliptic cone grid with both Cartesian and cylindrical coordinate systems. $(330 \times 40 \times 30)$. . . . . . . . . .

2.2 Density contours for Mach 7.93 air flow around a sharp-nose elliptic

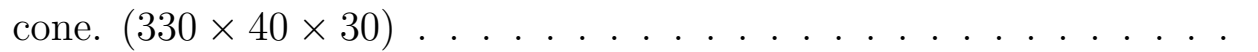

2.3 Grid independence study for Mach 7.93 air flow around a sharp-nose

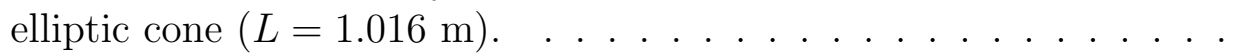

2.4 Nondimensional pressure distributions for Mach 7.93 air flow around a sharp-nose elliptic cone ( \pm 5 percent experimental uncertainty). PNS calculations and experimental data from [71]. . . . . . . . .

2.5 Stanton number distributions for Mach 7.93 air flow around a sharpnose elliptic cone (symbol size reflects \pm 10 percent experimental uncertainty). PNS calculations and experimental data from [71]. . . . 26

2.6 Blunt-nose elliptic cone geometry. Adapted from [74]. . . . . . . . . 26

2.7 Grid independence study for Mach 14.2 air flow around a blunt-nose elliptic cone $(L=0.21 \mathrm{~m})$. . . . . . . . . . . . . . . . . . . 28

2.8 Pressure contours for Mach 14.2 air flow around a blunt-nose elliptic

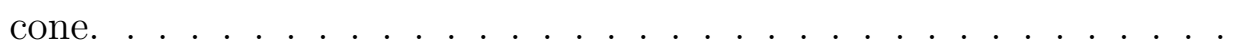


2.9 Pressure coefficient distributions for Mach 14.2 air flow around a blunt-nose elliptic cone $( \pm 7$ percent experimental uncertainty). Experimental data from Ref. [74]. . . . . . . . . . . . . . . . . .

2.10 Stanton number distributions for Mach 14.2 air flow around a bluntnose elliptic cone (symbol size reflects \pm 4.5 percent experimental uncertainty). Experimental data from Ref. [74]. . . . . . . . . . . . 32

2.11 Temperature contours for Mach 7.93 air flow around a sharp-nose elliptic cone with the 'interior walls.' (before Post-LeMANS) . . . . 34

2.12 Temperature contours for Mach 7.93 air flow around a sharp-nose elliptic cone without the 'interior walls.' (after Post-LeMANS) . . .

3.1 Geometry and grid for validation of volumetric energy deposition in a free domain. . . . . . . . . . . . . . . . . . . .

3.2 Temperature contours for Mach 3.1 nitrogen flow along the centerline of a free domain $(y=0)$, with a spherical energy deposition $Q=100$ W. $(40 \times 40 \times 40) \ldots \ldots \ldots \ldots \ldots$

3.3 Steady flight (vehicle trim), is achieved by extending the flaps a small angle for the F-22 Raptor, a supersonic fighter aircraft [81]. .

3.4 Grid independence study for Mach 12.6 air flow around a blunt-nose

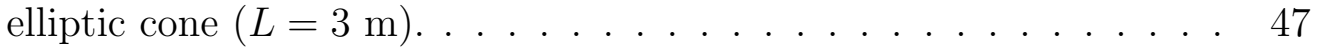

3.5 Blunt-nose elliptic cone geometry with an extended $2^{\circ}$ flap. . . . . . 48

3.6 Pressure contours for Mach 12.6 air flow around a blunt-nose elliptic geometry with $2^{\circ}$ flare $2.8 \mathrm{~m}$ from the leading edge. . . . . . . . . .

3.7 Three characteristic length scales $(3 \lambda)$, are used to determine $z_{c}$ and $\phi$ for the phenomenological energy deposition study. . . . . . . .

3.8 Contours of constant $S$ for the various ellipsoid deposition shapes employed for Mach 12.6 air flow around a blunt-nose elliptic cone geometry $(L=3 \mathrm{~m}) . \ldots \ldots \ldots \ldots$

3.9 Pressure coefficient and Stanton number distributions along the top centerline $\left(\theta=0^{\circ}\right)$, for Mach 12.6 air flow around a blunt-nose elliptic cone $(L=3 \mathrm{~m})$, for various energy deposition patterns $\left(\mathrm{T}_{\mathrm{w}}=300\right.$ $\mathrm{K}) \ldots \ldots \ldots \ldots \ldots \ldots \ldots$ 
3.10 Normalized change in pitching moment for Mach 12.6 air flow around a blunt-nose elliptic cone $(L=3 \mathrm{~m})$, for various energy deposition patterns $\left(\mathrm{T}_{\mathrm{w}}=300 \mathrm{~K}\right) \ldots \ldots \ldots \ldots \ldots \ldots . \ldots \ldots$

3.11 Radiative equilibrium surface temperature contours for Mach 12.6 air flow around a blunt-nose elliptic cone $(L=3 \mathrm{~m}) \ldots \ldots \ldots \ldots$

3.12 Normalized change in pitching moment for Mach 12.6 air flow around a blunt-nose elliptic cone $(L=3 \mathrm{~m})$, with an oblate spheroid energy deposition for two isothermal walls and a radiative equilibrium wall.

3.13 Pressure coefficient and Stanton number distributions along the top center line $\left(\theta=0^{\circ}\right)$, for Mach 12.6 air flow around a blunt-nose elliptic cone $(L=0.62 \mathrm{~m})$, with different amounts of energy deposition $\left(\mathrm{T}_{\mathrm{w}}=300 \mathrm{~K}\right) \ldots \ldots \ldots \ldots \ldots \ldots$

3.14 Temperature contours for Mach 14.2 air flow around a blunt-nose elliptic cone $(L=0.21 \mathrm{~m})$, with an oblate spheroid energy deposition $Q=1 \mathrm{~kW} \ldots \ldots \ldots \ldots \ldots \ldots \ldots \ldots$

3.15 Pressure coefficient and Stanton number distributions along the top center line $\left(\theta=0^{\circ}\right)$, of Mach 14.2 air flow around a blunt-nose elliptic cone $(L=0.21 \mathrm{~m})$, with various levels of energy deposition $\left(\mathrm{T}_{\mathrm{w}}=294 \mathrm{~K}\right) \ldots \ldots \ldots \ldots \ldots \ldots \ldots$

3.16 Moment coefficient versus the nondimensional total power deposition $(\tilde{Q})$, for various vehicle configurations $\left(\mathrm{T}_{\mathrm{w}} \simeq 300 \mathrm{~K}\right.$, thermal equilibrium, 5 sp., oblate spheroid deposition). . . . . . . . . .

4.1 An illustration of the nonuniform finite difference methodology used to find the flux through face $i$ from $i-1$ to $i+1$. ( $\phi$ is known at $i+1$ [blue] and unknown at $i-1$ [red]) . . . . . . . . .

4.2 Rectilinear grids used in the analytical MHD validation exercise. $(20 \times 20 \times 20) \ldots \ldots \ldots \ldots \ldots \ldots \ldots$

4.3 Contours of the electric potential $(\phi)$, for the analytical MHD validation case. The top half of the domain is populated by computed values while the bottom is theoretical. $(20 \times 20 \times 20) \ldots \ldots .78$

4.4 Nonuniform grid for current flow between parallel electrode plates.

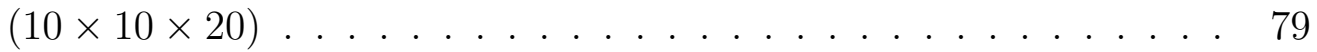


4.5 Various electrical conductivity models applied to flow between parallel electrode plates. . . . . . . . . . . . . . . . 80

4.6 Contours of the electric potential $(\phi)$, for parallel electrodes with no flow $\left(\sigma=1 \Omega^{-1} \mathrm{~m}^{-1}\right)$. The left side of the domain is composed of computed values while the right side is theoretical. $(10 \times 10 \times 20)$.

4.7 Electric potential distributions between parallel electrode plates with no flow for two electrical conductivity models. The distribution is extracted along the $x=0.5 \mathrm{~m}$ and $y=0.5 \mathrm{~m}$ ray. . . . . . . .

4.8 Electric potential contours between parallel electrode plates $(\sigma=$ $1 \Omega^{-1} \mathrm{~m}^{-1}$ and $\mathbf{u}=0$ ). The left side of the domain is composed of computed values using four processors, while the right side is the theoretical result. $(20 \times 20 \times 40) \ldots \ldots \ldots \ldots \ldots$

4.9 Geometry for a power-law shaped blunt-leading edge $z=x^{0.5}$. $(60 \times$ $30 \times 20) \ldots \ldots \ldots \ldots \ldots \ldots \ldots \ldots$

4.10 Coefficient of pressure and nondimensional heat flux along the center stagnation line $(y=0.025 \mathrm{~m})$, for Mach 12.6 air flow around a powerlaw blunt leading-edge for various grids. . . . . . . . . . . . .

4.11 Temperature contours for Mach 12.6 air flow around a power-law blunt leading-edge from coarse $(30 \times 15 \times 10)$, and fine $(120 \times 60 \times 40)$, grids. . . . . . . . . . . . . . . .

4.12 Speed up versus number of processors for flow solver with and without the MHD routine. . . . . . . . . . . . . .

4.13 Schematic of the channel flow with finitely segmented electrodes. Units are in meters. . . . . . . . . . . . . . . . . . . 9 90

4.14 Geometry and boundary conditions for the 3D channel flow with finitely segmented electrodes. $(50 \times 20 \times 4) \ldots \ldots \ldots 1$

4.15 Cartoon of a five point stencil used for period boundary conditions. 91

4.16 Electric potential $(\phi)$, between two segmented electrodes at two different locations $(x=0 \mathrm{~m}$ and $0.25 \mathrm{~m})$, for various grids. $(z=0$

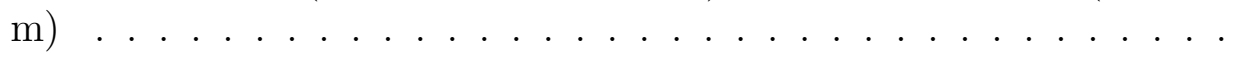


4.17 Electric potential contours for the segmented electrode channel without a magnetic field and constant electrical conductivity. $\quad(\mathbf{B}=$ $\left.0, \sigma=1 \Omega^{-1} \mathrm{~m}^{-1}\right) \ldots \ldots \ldots \ldots \ldots \ldots$

4.18 Current density streamlines $\mathbf{j}$ between two segmented electrodes with a magnetic field $\left(\mathrm{B}_{z}=1 \mathrm{~T}\right)$, constant electrical conductivity $(\sigma=$ $\left.1 \Omega^{-1} \mathrm{~m}^{-1}\right)$, and a streamwise velocity $(\mathbf{u}=f(y)) .(100 \times 40 \times 8)$.

5.1 Electron temperature versus the normalized electric field for various degrees of ionization in 7 species air $\left(\mathrm{N}_{2}, \mathrm{O}_{2}, \mathrm{NO}, \mathrm{N}, \mathrm{O}, \mathrm{NO}^{+}\right.$, and e).100

5.2 Electrical conductivity of equilibrium air for various temperatures and pressures, reproduced from Sutton and Sherman [93] . . . . . . 102

5.3 Mole fractions for 11 species equilibrium air versus temperature for two pressures. . . . . . . . . . . . . . 106

5.4 Electrical conductivity versus temperature from various models for two pressures. . . . . . . . . . . . . . . 107

5.5 Electrical conductivity and temperature of equilibrium air $(p=0.001$ atm), for various shock Mach numbers. Reproduced from Lamb and Lin [116]. ( \pm 20 percent experimental uncertainty $)$. . . . . . . . 108

5.6 Electrical conductivity versus temperature for equilibrium air $(p=$ $0.001 \mathrm{~atm})$. Experimental measurements from [116]. (symbol size reflects \pm 20 percent experimental uncertainty) . . . . . . . . . . 109

5.7 Electrical conductivity of argon $(p=0.013 \mathrm{~atm})$, reproduced from Lin et al. [126]. . . . . . . . . . . . . . . . . . . . . . . . . 114

5.8 Design of Experiment 'learning' and 'testing' points for the threedimensional surrogate model of weakly-ionized argon. . . . . . . . 116

$5.9 \quad$ A spherical coordinate transformation representing a three species gas composition. The radius of the eighth of the sphere equals one. 118

5.10 Electrical conductivity contours for weakly-ionized argon. . . . . . . 119

5.11 Electron collision frequency for weakly-ionized argon at $p=1$ atm. . 120

5.12 Various PRS model surfaces for weakly-ionized argon. . . . . . . . . 121

5.13 Error metrics for several PRS models of weakly-ionized argon. . . . 125 
5.14 Global sensitivity analysis of several surrogate models of weaklyionized argon using a Monte Carlo technique with fifty thousand iterations. . . . . . . . . . . . . . . . . . 127

5.15 Average CPU-cost for executing various electrical conductivity models.129

5.16 Design of Experiment 'learning' and 'testing' points for the elevendimensional surrogate model of weakly-ionized air. . . . . . . . . . 132

5.17 Error metrics for several PRS models of weakly-ionized air. . . . . . 134

5.18 Global sensitivity analysis of several surrogate models of weaklyionized air using a Monte Carlo technique with fifty thousand iterations. . . . . . . . . . . . . . . . . . . . . . 135

5.19 Design of Experiment 'learning' and 'testing' points for the elevendimensional surrogate model of weakly-ionized air in a reduced design space. . . . . . . . . . . . . . . . . . 136

5.20 Temperature contours for Mach 12.6 air flow around a blunt-nose elliptic cone geometry $(L=3 \mathrm{~m}) . \ldots \ldots \ldots$

5.21 Magnetic field contours and streamlines for a blunt-nose elliptic cone geometry with a dipole magnet. . . . . . . . . . . . . . . 139

5.22 Electrical conductivity contours for Mach 12.6 air flow around the stagnation region of a blunt-nose elliptic cone geometry $(L=3 \mathrm{~m})$, for various conductivity models. $(\mathbf{E}=0) \ldots 140$

5.23 Magnetic force contours for Mach 12.6 air flow around the stagnation region of a blunt-nose elliptic cone geometry $(L=3 \mathrm{~m})$, for various conductivity models with a $0.2 \mathrm{~T}$ peak strength dipole magnetic field.141

6.1 Glow discharge on a flat plate in Mach 5.2 air flow with a potential difference of $2800 \mathrm{~V}$ (50 mA). Image from Kimmel et al. [28]. . . . . 145

6.2 Geometry and placement of flush-mounted electrodes for a powerlaw shaped $\left(z=x^{0.5}\right)$, blunt leading-edge geometry. . . . . . 146

6.3 Thermal nonequilibruim temperature contours for Mach 12.6 air flow around a blunt leading-edge geometry. . . . . . . . . . . . 147 
6.4 Electric potential contours and current lines for flow between flushmounted electrodes with a $10 \mathrm{~V}$ potential difference for Mach 12.6 air flow around in a blunt leading-edge geometry. (Raizer's conductivity

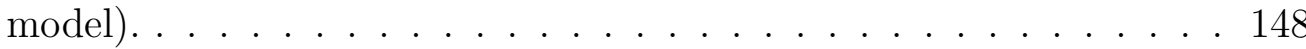

6.5 Thermal energy deposition due to an applied voltage between a cathode and anode flush-mounted in a blunt leading-edge geometry. (Raizer's conductivity model) . . . . . . . . . . . . . . . . . . 149

6.6 Infrared photographs of hemisphere capped geometry with and without an applied-magnetic field. Image from Gülhan et al. [30]. . . . . 150

$6.7 \quad$ Hemisphere capped geometry. Adapted from [16]. . . . . . . . . . 151

6.8 Nondimensional pressure and heat flux along the surface of Mach 4.75 argon flow around a hemisphere capped geometry for various grids. . . . . . . . . . . . . . . . . . . . 153

6.9 Temperature contours for Mach 4.75 argon flow around a hemisphere capped geometry. $(\alpha=0.00623) \ldots \ldots \ldots \ldots \ldots$

6.10 Nondimensional dipole magnetic field contours from a magnet located in the hemisphere capped geometry. . . . . . . . . . . 156

6.11 L2 residual error from a simulation of Mach 4.75 argon flow around a hemisphere capped geometry with a $0.13 \mathrm{~T}$ magnet. (Chapman and Cowling conductivity model $) \ldots \ldots \ldots$

6.12 Temperature contours and current lines for Mach 4.75 argon flow around a hemisphere capped geometry with a $0.28 \mathrm{~T}$ magnetic field. Chapman and Cowling conductivity model) . . . . . . . . . . 158

6.13 L2 residual error from the MHD module of a simulation of Mach 4.75 argon flow around a hemisphere capped geometry with a 0.28 T magnet. (Chapman and Cowling conductivity model) . . . . . . 159

6.14 Density ratio contours for Mach 4.75 argon flow around a hemisphere capped geometry for various electrical conductivity models. $\left(\mathrm{B}_{\max }=\right.$ $0.13 \mathrm{~T}) \ldots \ldots \ldots \ldots \ldots \ldots \ldots \ldots \ldots \ldots$

6.15 Percent change in shock standoff distance versus magnetic field strength for Mach 4.75 argon flow around a hemisphere capped geometry with various electrical conductivity models. Measurements from [16]. (experimental uncertainty $\pm 10 \%) \ldots \ldots \ldots \ldots$ 
6.16 Heat flux contours for Mach 4.75 argon flow around a hemisphere capped geometry with a $0.13 \mathrm{~T}$ magnetic field and various electrical conductivity models. . . . . . . . . . . . . . . . . 162

A.1 Diagram of the methodology for partitioning the mesh within LeMANS. . . . . . . . . . . . . . . . . 179

A.2 Cartoon of the methodology for partitioning the mesh in the PreLeMANS code. . . . . . . . . . . . . . . . . 180

B.1 Methodology of a systematic search in the Post-LeMANS code. . . . 183

B.2 Illustration of cube storage of the output files. . . . . . . . . . 185

B.3 Cartoon explaining the cubic systematic search routine in the PostLeMANS code. . . . . . . . . . . . . . . . 186

B.4 Distribution of 8 output files to 3 processors for the Post-LeMANS code. . . . . . . . . . . . . . . . . . 187

B.5 Time and processor load for 8 output files and 3 processors for the Post-LeMANS code. . . . . . . . . . . . . . . . . . 188

B.6 Speed-up versus number of processors for the Post-LeMANS code with 8 output files. . . . . . . . . . . . . . . . . . 189 


\section{LIST OF TABLES}

\section{Table}

2.1 Flow conditions for the sharp-nose elliptic cone experiment conducted by Kimmel et al. $[70,71]$. . . . . . . . . . . . . . . . . . 20

2.2 Flow conditions from Run 15 of the blunt-nose elliptic cone experiment conducted by Nowlan et al. [74]. . . . . . . . . . . . . . . 27

3.1 Flow-field conditions for the volumetric heating validation case in a free domain. . . . . . . . . . . . . . . . . . . . . . 41

3.2 Power loss through the free domain boundaries for $Q=100 \mathrm{~W}$. . . 43

3.3 Flow conditions for Mach 12.6 air flow at an altitude of $40 \mathrm{~km}$. . . 45

3.4 Ellipsoid deposition parameters for Mach 12.6 air flow around a blunt-nose elliptic cone $(L=3 \mathrm{~m})$. . . . . . . . . . . . . . . . . 49

3.5 Ellipsoid deposition parameters for Mach 12.6 air flow around bluntnose elliptic cone $(L=0.62 \mathrm{~m})$. . . . . . . . . . . . . . . . 58

3.6 Properties of the U.S. Standard Atmosphere [85]. . . . . . . . . . 60

3.7 Ellipsoid deposition parameters for Mach 14.2 air flow around bluntnose elliptic cone $(L=0.21 \mathrm{~m})$. . . . . . . . . . . . . . 61

3.8 Reference area for various the blunt-nose elliptic cone geometries. . 63

4.1 Boundary conditions for the MHD solver . . . . . . . . . . 75

5.1 Experimental measurements of electrical conductivity in air at $p=$ 1 atm from [113]. ( \pm 20 percent experimental uncertainty $)$. . . . . 104

5.2 Computational cost (CPU-seconds) to determine the electrical conductivity for ten thousand finite-volume cells . . . . . . . . . 109 
5.3 Total number of constant coefficients for an $n^{\text {th }}$ order polynomial in $k$ dimensions (Eqn. 5.12). . . . . . . . . . . . . . . . . 112

$5.4 \quad$ Definitions of error metrics $(\hat{y}=$ predicted, $y=$ actual, $n$ points $) \ldots 123$

5.5 Example scenarios to demonstrate the error definitions. . . . . . . . 123

5.6 Error metrics for weakly-ionized argon. . . . . . . . . . . . . . . 124

5.7 Model accuracy for weakly-ionized argon with reduced dimensions. $\left(\mathrm{E} / N, \chi_{\mathrm{Ar}}\right.$, and $\left.\chi_{\mathrm{Ar}^{+}}\right) \ldots \ldots \ldots \ldots \ldots \ldots \ldots$

5.8 Model accuracy for weakly-ionized air with and without a reduced design space. . . . . . . . . . . . . . . . . . . 137

5.9 Total magnetic force produced by a $0.2 \mathrm{~T}$ dipole magnet field centered in a blunt-nose elliptic cone geometry $(L=3 \mathrm{~m})$, in March 12.6 air. . . . . . . . . . . . . . . . . . . . 142

6.1 Flow conditions for the MHD-Heat Shield experiment as reported by Kranc et al. [16] . . . . . . . . . . . . . . . . . . . . . . . . . 151

6.2 Modifications to the freestream conditions for the MHD-Heat Shield experiment of Kranc et al. [16]. . . . . . . . . . . . . . . . . . . . 154

6.3 Electrical conductivity estimates for the MHD-Heat Shield experiment without an applied magnetic field. $(\mathrm{E} / N=0) \ldots \ldots 156$

6.4 Percent change in heat flux to the surface for Mach 4.75 argon flow around a hemisphere capped geometry with an MHD-Heat Shield. . 163

C.1 The pitching moment for the 'small' blunt elliptic cone simulations with freestream conditions listed in Table 2.2 and deposition shape parameters listed in Table 3.7. $\left(L=0.21 \mathrm{~m}, \mathrm{~T}_{w}=294 \mathrm{~K}\right) \ldots \ldots$.

C.2 The pitching moment for the 'large' blunt elliptic cone simulations with freestream conditions listed in Table 3.3 and deposition shape parameters listed in Table 3.4. $\left(L=3 \mathrm{~m}, \mathrm{~T}_{w}=300 \mathrm{~K}\right) \ldots \ldots$. .

C.3 The pitching moment for the 'large' blunt elliptic cone simulations with freestream conditions listed in Table 3.3 and deposition shape parameters listed in Table 3.4. $\left(L=3 \mathrm{~m}, \mathrm{~T}_{w}=1000 \mathrm{~K}\right) \ldots \ldots . .193$ 
C.4 The pitching moment for the 'large' blunt elliptic cone simulations with freestream conditions listed in Table 3.3 and deposition shape parameters listed in Table 3.4. ( $L=3 \mathrm{~m}, \mathrm{~T}_{w}=$ fully radiative $) .$.

C.5 The pitching moment for the 'medium' blunt elliptic cone simulations with freestream conditions listed in Table 3.3 and deposition shape parameters listed in Table 3.5. $\left(L=0.62 \mathrm{~m}, \mathrm{~T}_{w}=300 \mathrm{~K}\right) \ldots \ldots$

D.1 Temperature, total number density, and mole fractions for equilibrium air $\left(p=10^{-3} \mathrm{~atm}\right)$, computed using the computational code in [115]

D.2 Temperature, total number density, and mole fractions for equilibrium air ( $p=1 \mathrm{~atm})$, computed using the computational code in [115]

F.1 Examples of scaling the normalized electric field. . . . . . . . . 200

F.2 Constant coefficients for three species argon ( $3^{\text {rd }}$ Order PRS).

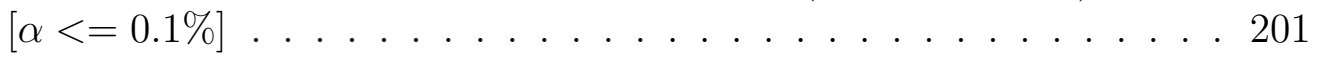

F.3 Constant coefficients for eleven species air ( $2^{\text {nd }}$ Order PRS $)$.

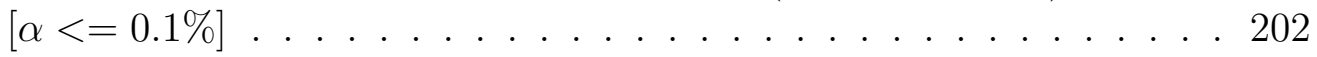

F.4 Constant coefficients for eleven species air ( $3^{\text {rd }}$ Order PRS).

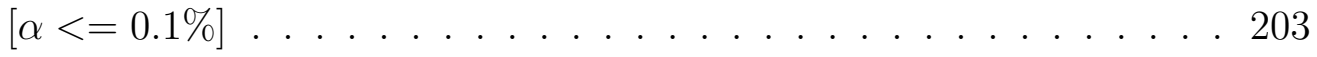




\section{LIST OF APPENDICES}

\section{Appendix}

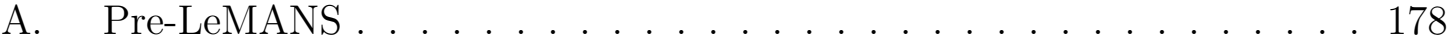

A.1 Methodology . . . . . . . . . . . . . . . . 179

A.2 Modified LeMANS . . . . . . . . . . . . . . . . . . . 180

B. Post-LeMANS . . . . . . . . . . . . . . . . . 182

B.1 Search Algorithms . . . . . . . . . . . . . . . . . . 183

B.2 Parallelization . . . . . . . . . . . . . 186

B.3 Conclusions . . . . . . . . . . . . . . . . . . . 189

C. Phenomenological Results . . . . . . . . . . . . . . . 191

D. Air - Mole Fractions . . . . . . . . . . . . . . . . . . . . . 194

E. Air - Mole Fraction Angles . . . . . . . . . . . . . . . . . . . . . 198

F. Polynomial Response Model . . . . . . . . . . . . . . . . . 199

F.1 Variables . . . . . . . . . . . . . . . . . . . . . . 199

F.2 Constant Coefficients . . . . . . . . . . . . . . . . 200 


\section{LIST OF NOMENCLATURE}

\section{Physical Constants}

$\begin{array}{ll}e & \text { Elemental charge, } 1.6022 \times 10^{-19} \mathrm{C} \\ \epsilon_{0} & \text { Permittivity of free space, } 8.85 \times 10^{-12} \mathrm{~F} / \mathrm{m} \\ k & \text { Boltzmann constant, } 1.3807 \times 10^{-23} \mathrm{~J} / \mathrm{K} \\ m_{\mathrm{e}} & \text { Electron mass, } 9.11 \times 10^{-31} \mathrm{~kg} \\ \mu_{0} & \text { Permeability of free space, } 4 \pi \times 10^{-7} \mathrm{~N} / \mathrm{A}^{2} \\ R & \text { Universal gas constant, } 8314 \mathrm{~J} / \mathrm{kmol} \mathrm{K} \\ \sigma^{*} & \text { Stefan-Boltzmann constant, } 5.6704 \times 10^{-8 \mathrm{~W} / \mathrm{m}^{-2} \mathrm{~K}^{-4}}\end{array}$

\section{Acronyms}

$\begin{array}{ll}\text { CFD } & \text { Computational Fluid Dynamics } \\ \text { CG } & \text { Center of Gravity } \\ \text { DOE } & \text { Design of Experiments } \\ \text { ePE } & \text { Exponential Percent Error } \\ \text { FVS } & \text { Flux Vector Splitting } \\ \text { GB } & \text { Gigabyte } \\ \text { LeMANS } & \text { The Michigan Aerothermal Navier-Stokes Solver } \\ \text { LEO } & \text { Low Earth Orbit } \\ \text { MAE } & \text { Mean Absolute Error } \\ \text { MHD } & \text { MagnetoHydroDynamics } \\ \text { MPI } & \text { Message Passing Interface } \\ \text { NASA } & \text { National Aeronautics and Space Administration } \\ \text { ODE } & \text { Ordinary Differential Equation } \\ \text { PE } & \text { Percent Error } \\ \text { PNS } & \text { Parabolized Navier-Stokes } \\ \text { PRS } & \text { Polynomial Response Surface } \\ \text { RAM } & \text { Random Access Memory } \\ \text { RMSE } & \text { Root Mean Square Error } \\ \text { TBS } & \text { Thermal Protection System } \\ \text { VHS } & \text { Variable Hard Sphere model }\end{array}$




\section{CHAPTER I}

\section{Introduction}

The main objective of this dissertation is to explore the feasibility of flight control and thermal shielding for hypersonic vehicles using methods based on electromagnetic flow control. Since experimental testing of hypersonic configurations is extremely expensive, potentially dangerous, and very limited due to existing ground facilities constraints, this dissertation will rely on computational methods to investigate these technologies for representative hypersonic configurations. As a result, this dissertation improves upon the numerical simulation tools previously available to model hypersonic, non-equilibrium gas flows with electromagnetic effects.

This research is motivated, in part, by the recent resurgence of interest in using plasma-based flow control devices to enhance or even replace existing systems in hypersonic configurations (e.g., aerodynamic control, propulsion, drag reduction, communications, etc.). This interest is due, in part, to the increasing demand for rapid access to space and the desire for sustained hypersonic flight, both in commercial and national defense applications. In addition, significant advances in materials, manufacturing techniques, and mechanical systems over the past half-century motivate further exploration into the usefulness of these technologies in hypersonic applications. 
Near-Space is a region of the atmosphere between the limit of controlled commercial airspace and low earth orbit (LEO) [1]. This region of the atmosphere is considered by many to provide the most suitable conditions for viable plasma-based technologies because the freestream density is significantly lower than at sea-level, which reduces drag on the vehicle (allowing it to more easily obtain hypersonic speeds).

This dissertation focuses on hypersonic configurations because high velocity is necessary to achieve rapid access to space and rapid global reach. The large freestream kinetic energy dissociates and ionizes the freestream air as it passes through the vehicle's bow shock. Plasma technologies will generally be more effective in flows with high levels of natural ionization and thus electrical conductivity. Thus, these technologies appear more promising when applied to hypersonic systems because the gas already has an appreciable level of electrical conductivity and may not need to rely on 'seeding' the freestream (although that concept may provide additional benefits).

Unfortunately, the high speeds required to maintain sufficient flight velocity for high levels of natural electrical conductivity are currently only available by rocket propulsion, except for the successful test flights of the X-43, which only operated for 10 seconds at Mach 7 and Mach 10 [2], and rail gun technology, which has been successful at launching small artillery shells ( $\sim 3 \mathrm{~kg}$ aluminum slugs), at about half of orbital speeds [3]. However, the rail gun technology does not involve sustained propulsion since the projectile's energy and momentum decay as soon as it leaves the launcher.

The main challenge with maintaining sufficient velocity is reducing the high drag experienced on the vehicle. Air-breathing propulsion requires large air-inlets to draw sufficient density inside the combustion chamber. These inlets create substantial 
skin-friction due to the large surface area. Configurations which rely on rocket or rail gun technology to provide propulsion also incur significant losses in performance because leading edges cannot be made sharp enough to minimize drag because of erosion by hot hypersonic flow.

Figure 1.1 illustrates the 'near-space' region of the atmosphere. As previously mentioned, this dissertation considers 'near-space' to be the region in the atmosphere most suitable for plasma-based technologies on hypersonic configurations, so Mach numbers are hypersonic (Mach $\gtrsim 5)$, but do not have sufficient velocity or altitude to obtain LEO (Mach $\gtrsim 25$ and altitude $\gtrsim 150 \mathrm{~km}$ ).

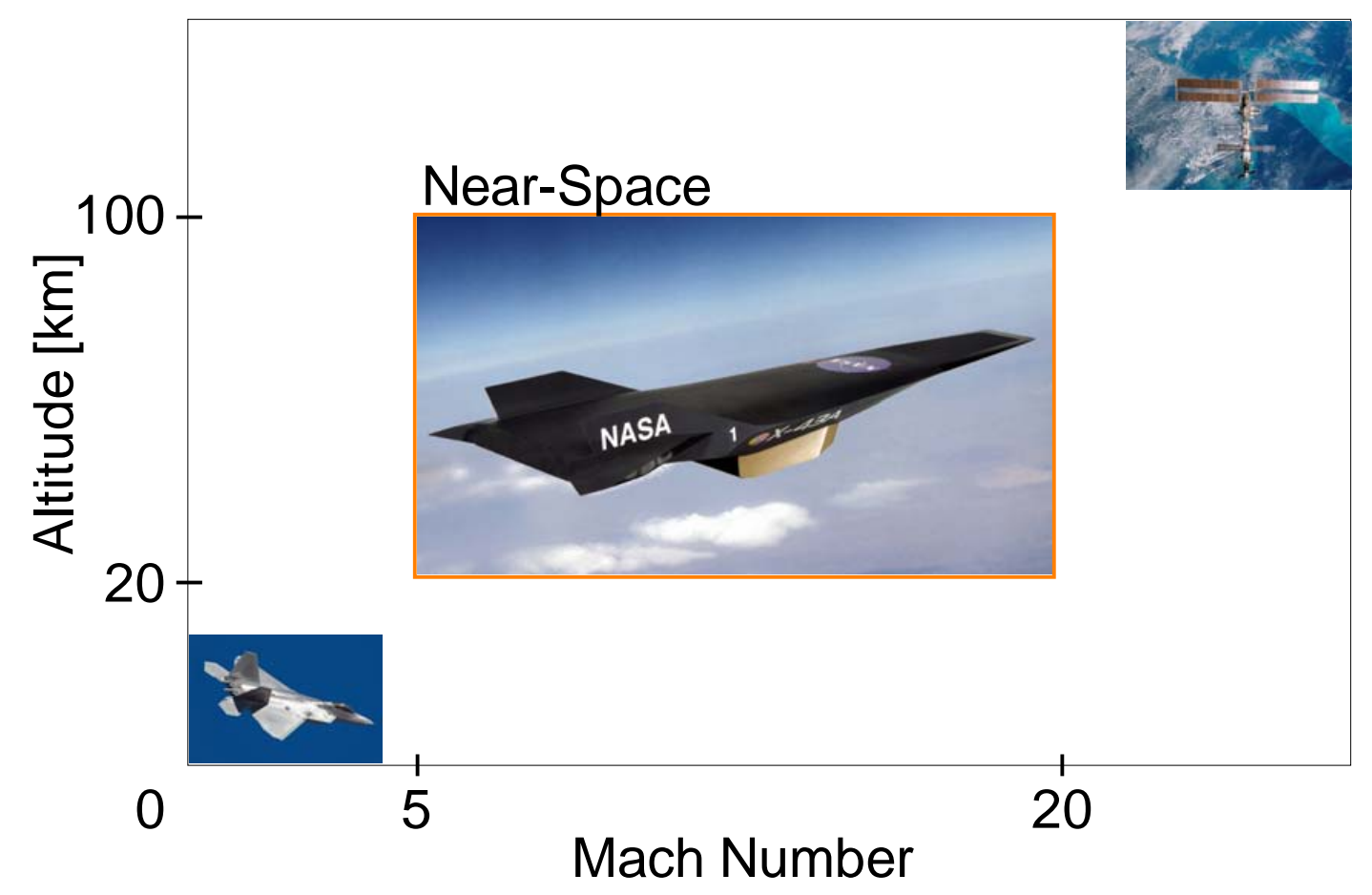

Figure 1.1: The 'near-space' region of the atmosphere. Images from left to right: US Air Force photos (http://www.af.mil, image: 080204-F-1001W030), NASA Dryden photo collection (http://www.dfrc.nasa.gov, image: ED99-45243-01), NASA's image of the day (http://www.nasa.gov, image: 19$)$ 


\subsection{Review of Related Work}

While the Navier-Stokes equations were presented in the early to mid-1800's (Navier - 1827, Stokes - 1845) [4], and the governing equations for electric and magnetic fields were first presented by Maxwell in 1861 [5], it was not until 1942 that Alfvén first combined the two fields (i.e., fluid mechanics and electromagnetism), to form the field of magnetohydrodynamics (MHD) [6]. Hypersonic flight took another decade, but it is not surprising that the first research applying electromagnetic technologies to hypersonic fluids followed shortly thereafter. One of the first plasma technologies to be coupled with hypersonic flow control was presented in the late 1950's when Kantrowitz [7] and Resler and Sears [8, 9] explored the idea of using an applied magnetic field to reduce the heat transfer to a hypersonic vehicle. They conducted some of the first calculations demonstrating the potential benefits an applied magnetic field may have on an incoming weakly-ionized flow, a condition typically observed during re-entry. The magnetic field, if properly aligned, creates a magnetic force which opposes the incoming flow, effectively increasing the shock standoff distance. The thickening of the shock layer reduces the gradients near the stagnation point, and thus lowers the peak heat transfer rate.

In the midst of the space race, this novel idea attracted a lot of attention as many groups looked to further explore and refine the semi-analytical calculations by making various approximations to the conservation equations. Of these efforts, the work by Bush $[10,11]$ is considered to be one of the most complete approximate ana-

lytic solutions [12]. Bush's approach used a local solution at the stagnation point of the hypersonic flow over an axisymmetric blunt body, and predicted significant flow deceleration with the presence of a magnetic field. The first modern computational 
fluid dynamic (CFD) simulations of the MHD blunt body problem were completed about a decade later by Coakley and Porter [13]. Because of the lack of computational resources at the time, the simulations still required significant simplifications, including assuming the gas was ideal, non-reacting, and inviscid.

The first experimental work to complement this computational activity was completed by Ziemer [14] and focused on measuring the shock standoff distance. Bush's approximate results were in reasonable agreement with these first experiments. The first heat transfer measurements were collected in the experimental work by Wilkinson [15] for Mach 3 ionized argon at the stagnation point of a blunt cone.

Another experimental effort was conducted by Kranc et al. [16] in the late 1960's. This work provided additional experimental validation sets for the continuing computational efforts, as it explored shock standoff distance and heat transfer mitigation for hypersonic flow over two different axisymmetric geometries. These experiments were run in a flow regime where both the viscosity and Hall effect are important, and confirmed the increase in the shock standoff distance and an increase in total drag on the geometry in the presence of a dipole magnetic field. The experiments also exhibited an increase in total heating, which has been attributed to the Hall effect $[17,18]$. This was unexpected because the thickening of the shock layer reduces gradients within the stagnation region, which should reduce the heat flux to the body. Previous semi-analytic work had predicted that the Hall effect would only reduce the effectiveness of the magnetic force on increasing the shock standoff distance and total drag [19]. Regardless of this unexpected outcome, it was determined that the large magnetic field strength needed to make the technology practical required a magnet that was too heavy to be placed on re-entry vehicles and the research area faded [20].

While hypersonic research continued to experience strong support through the 
rest of the twentieth century due, in part, to various National Aeronautics and Space Administration (NASA) sponsored programs like Apollo and Shuttle [21], it was not until the mid-1990's that interest in plasma-assisted hypersonic flow control started to reappear $[22,23,24]$. This resurgence, as previously mentioned, has been credited to many factors including the increasing demand for sustained hypersonic flight, rapid access to space, and numerous mechanical and material advances in the area of flight-weight MHD technologies. One of the first to reevaluate the technology using modern CFD was Palmer [25], who performed first-order spatially accurate simulations of the time-dependent Maxwell's equations coupled to the Navier-Stokes equations to analyze a Mars return vehicle.

Despite the large financial costs, limited facilities, and technical challenges, some recent experimental studies have been performed by Lineberry et al. [26], Takizawa et al.[27], Kimmel et al. [28], Matsuda et al. [29], and Gülhan et al. [30] to explore electromagnetic effects on hypersonic flows. While these efforts have provided new insight into electromagnetic phenomena in hypersonic flows, more precise measurements, and additional validation exercises for testing the accuracy of fluid-MHD codes, the rising costs (increased maintenance for aging facilities and additional safety protocol), associated with conducting hypersonic experiments greatly limits the number of experiments being conducted. In addition, available computing systems continue to experience exponential performance increases with substantial decreases in cost, which has led to a continued increase in computational research. In fact, Padilla estimates that if current trends continue, over $70 \%$ of hypersonic research will involve computational analysis by 2020 [21].

While the shift toward computational analysis for design and optimization of hypersonic vehicles allows designers to test a larger range of design variables with 
increasing geometric complexity, aerodynamic control and drag reduction are still major challenges. Therefore, a large amount of the recent plasma-based research has focused on hypersonic plasma interactions and plasma flow control technologies in order to explore ways of confronting these challenges [22, 23].

Minimizing drag for hypersonic vehicles leads to long thin bodies with sharp leading edges, which can limit the materials available for the vehicles thermal protection system (TPS). In addition, small defects in the production of the sharp leading edges can result in serious or even catastrophic problems for the TPS [31]. Blunting the leading edge reduces the manufacturing risk and increases the list of suitable materials available for the TPS, but results in a much larger wave drag [32]. Recent experimental and computational research by Shang et al. [33] has investigated ways of reducing drag on blunt nose bodies by means of plasma injection, while research by Kremeyer et al. [34] and Yan [35] focused on drag reduction and flow control using laser energy deposition (filamentation) ahead of conic and spherical hypersonic geometries.

Air-breathing propulsion requires air-inlets to reflect shocks into the isolator at a precise angle in order to properly condition the air (oxidizer) before it enters the combustion chamber. This requirement presents significant challenges for traditional mechanical flaps to control the inlet flow because the freestream conditions are not constant as the vehicle travels through the atmosphere, so the system must quickly adjust to accommodate the changing freestream conditions. In addition, the extreme conditions at the shock-inlet impingement point presents material challenges. Therefore, the concept of using energy deposition as a 'virtual cowl' for off-design scramjet engines has been studied using electron beam ionization by Macheret et al. [36] and with DC discharges by Shang et al. [37]. 
Aerodynamic control is traditionally accomplished using control surfaces (flaps) which are positioned away from the center of gravity to extend the maneuverability of the vehicle. The flap location is limited because the bow shock surrounding the vehicle will impinge on surfaces that extend beyond the shock envelope resulting in extreme pressure and heat transfer rates to the shock impingement point. As such, vehicle configurations tend to be streamlined with minimal protrusions from the fuselage, as seen in Fig. 1.2.

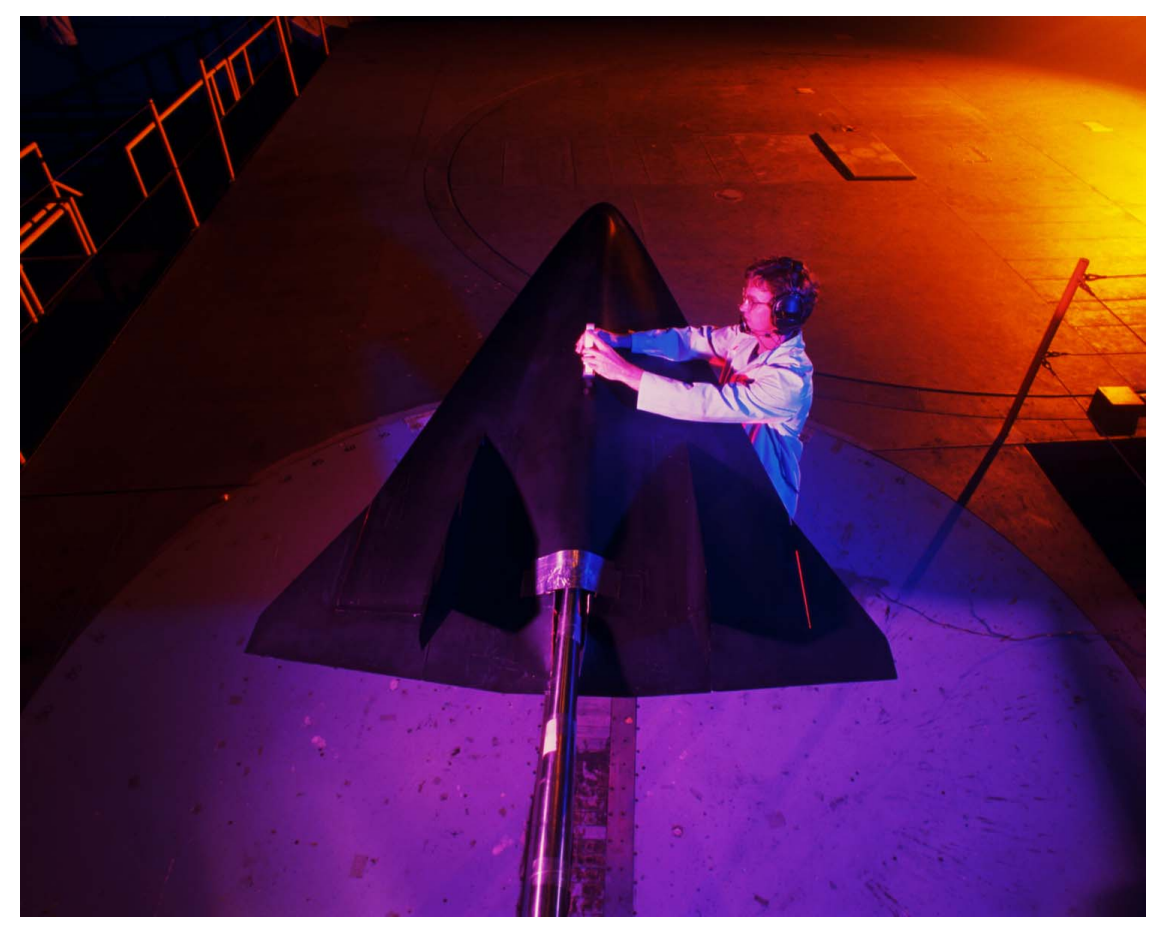

Figure 1.2: Mach 5.5 wave rider being prepared for a full scale wind tunnel test. (Great Images in NASA library, http://grin.hq.nasa.gov, image: GPN2000-001927)

Mechanically driven flaps require clearance below the surface of the flap to provide space for the flap control arm and a strong attachment point to push the flap from. In addition, there is a small gap in the TPS to allow for flap movement. This gap is difficult to protect and can cause heat related damage to the vehicle. Sustained cruise and other long duration hypersonic missions also suffer from nonuniform ablation of 
the flap, which results in a nonuniform control authority on the vehicle.

Plasma actuators provide several advantages because they do not have moving parts, they can be located either in or beneath the TPS, and are not constrained by the bow shock. This extends the range of possible locations for the actuator. In addition, they can potentially be turned on and off very rapidly, and should have a minimal aerothermal penalty when turned off. This list of benefits has motivated several computational studies to explore the applicability of plasma actuators in many hypersonic systems to provide steering moments [38, 39], changes in vehicle lift [40], control of flow separation [41, 42], and local heat load mitigation [43, 44, 45].

A majority of the recent computational research publicly presented focuses on the potential effects of energy deposition, though some have explored the use of magnetic fields to prevent communications blackout [46] and the possibility of MHD power extraction (i.e., on-board electrical power generation) $[47,48,49]$. While these areas also show promise, there are still limitations as to their applicability, because the magnets and power conditioning equipment required is still heavy [50], but not completely impractical.

While there are several groups heavily involved in this field of research, there are still many plasma technologies that are not fully understood nor adequately modeled. Many of the previously mentioned citations used two-dimensional or axisymmetric solvers to determine the resulting flow-field. While reducing spatial dimensions may be valid for the cases explored, most realistic hypersonic geometries are three-dimensional. In fact, Barmin et al. demonstrated the necessity of a threedimensional solution of the full MHD equations in order to prevent the introduction of unstable disturbances into the solution [51].

Another distinguishing feature between the recent computational studies is how 
they account for the electromagnetic effects. It is possible to solve Maxwell's equations directly coupled to the flow equations as shown by MacCormack [52] and D'Ambrosio [53]. However, the computational expense of obtaining a solution using this approach is extremely high since Maxwell's equations are significantly stiffer than the accompanying fluid equations (i.e., the time step required to solve Maxwell's equations limits the time step of the overall solution). As such, these methods have only been applied to simple two-dimensional and axisymmetric domains, and, currently, are limited to serial computing. Most of the computational work in the field accounts for electromagnetic effects by using the current continuity equation in the low magnetic Reynolds number approximation in a framework loosely coupled with the flow solver. This improves the time step limits imposed by Maxwell's equations, but also limits the types of problems that can be investigated.

Ultimately, the practical use of computational analysis for the design and development of hypersonic vehicles can only occur with computational tools that provide accurate solutions of the flow-field in a reasonable amount of time (i.e., hours or days). This requires the use of parallel computing systems. In addition, these tools must be able to accurately account for three-dimensional flows over complex geometries with thermodynamic nonequilibrium, finite-rate chemical reactions, accurate modeling of the gas transport properties, and appropriate modeling of the electromagnetic effects. This dissertation provides research toward this goal.

\subsection{Thesis Overview}

This chapter has presented the scope of this dissertation, and provided the motivation for the exploration of plasma-based technologies for hypersonic vehicles. With the main objectives established, the focus was restricted to explore configurations 
primarily existing in the 'near-space' region of the velocity-altitude map. Related research was summarized, with a particular emphasis on recent research being conducted by others in the field. This was done to demonstrate how this dissertation is related to these studies, extends the field's capabilities, and the understanding of electromagnetic effects in hypersonic flows.

Chapter II outlines the CFD code, LeMANS, which is used throughout this thesis. A significant portion of the chapter focuses on validation exercises to provide confidence in the solutions obtained from the flow solver over the flight regime of interest. In particular, simulations are performed for hypersonic laminar flow over three-dimensional sharp-and blunt-nose elliptic cone geometries. The elliptical geometries present additional complexity because the asymmetry around the circumference of the geometry produces a circumferential velocity component. Finally, the chapter summarizes the additional features and tools added to LeMANS as part of this thesis.

Chapter III summarizes a phenomenological heating model incorporated into the solver to investigate whether a practical level of pitching moment control can be achieved from volumetric energy deposition for a realistic hypersonic vehicle. Threedimensional simulations are performed for a blunt-nose elliptic cone geometry with volumetric energy deposition along the top-half of the geometry. The results are compared to results from a nominal geometry with a $2^{\circ}$ mechanical flap extension, suitable for generating trim pitching moments. A parametric study is performed to investigate how the shape, location, and amount of energy deposited affect the flow. In addition, various vehicle wall conditions are explored and conclusions made about the viability of volumetric energy deposition as a means for flow control in a hypersonic configuration. 
Since the heating model incorporated in Chapter III is phenomenological, its results near the location of the volumetric energy deposition may be unrealistic. In order to improve the physical modeling in that region and allow for broader exploration of electromagnetic effects on the flow, Chapter IV summarizes the development and coupling of a magnetohydrodynamics module to the fluid code. This module accurately estimates the electric and current density fields that exist in the flow by providing a solution to Ohm's law. Details of the model, its boundary conditions, and parallel implementation are discussed. In addition, several validation exercises are performed to assess the module's accuracy and parallel efficiency. Finally, the Hall effect is accounted for in the MHD routine through the introduction of an electrical conductivity tensor, and is validated by computing flow between segmented electrodes.

Traditionally, MHD solvers rely on relatively simple, semi-empirical models to predict the electrical conductivity, an important transport property needed to solve Ohm's law. While these models provide reasonable estimates within their range of validity, they are not general, and can be problematic for off-design simulations. They are particularly problematic when used for evaluation of plasma-based devices which may experience a large range of temperatures, pressures, and gas compositions.

Chapter V explores several existing electrical conductivity models and compares them to solutions of Boltzmann's equation, which provides a physically accurate estimate of the electric conductivity. The computational cost of directly linking Boltzmann's equation to the fluid code is prohibitive, so the majority of the chapter is spent outlining a general procedure for developing a surrogate model to solutions of Boltzmann's equation. Surrogate models of the electrical conductivity of weaklyionized argon and air are developed, presented, and discussed. Finally, the various 
electrical conductivity models, including the newly developed surrogate model, are applied to a representative geometry employing an MHD-Heat Shield concept to further illustrate the importance of using an appropriate electrical conductivity model when accounting for electromagnetic effects.

Chapter VI explores the applicability of two different types of plasma-based technologies by using the MHD module developed in Chapter IV and the electrical conductivity models outlined in Chapter V. The first is an arc discharge across two electrodes. The discharge causes Joule heating which can be used as a means of flow control through energy deposition, analogous to the problem studied in Chapter III. The second device explores the usefulness of an MHD-Heat Shield. A magnet located inside the forebody of the geometry provides a force to increase the bow shock standoff distance and decelerate the hot ionized particles as they approach the stagnation point.

Chapter VII summarizes the conclusions drawn in the previous chapters, and highlights the main contributions made to the field as a direct result of this work. The chapter concludes with an outline of recommended areas of future research, and lists additional capabilities that could be useful to the LeMANS code. 


\section{CHAPTER II}

\section{Navier-Stokes Solver}

The last half-century has seen a continuous growth in computational research, both in the development of new methods and the applications of these methods to various research areas, including fluid dynamics [54]. Computational fluid simulations provide an alternative to experiments for determining the effects of flows over bodies. They are motivated by the rising costs associated with conducting actual experiments (both ground-based and flight-tests), risk reduction by conducting the experiments within the confines of a computing system, and limited access to facilities which may not even be capable of conducting the experiment. This is particularly true in hypersonic research, where even the world's foremost ground facilities have severe geometry restrictions and short test duration capabilities.

Moore's Law predicts computer hardware performance will double every two years [55], and has been valid for the last 40 years. These breakthroughs in computing hardware translate to increased availability of relatively cheap computing resources which allows computational research to be conducted on a scale where hundreds, or even thousands, of design variations are quickly and accurately simulated, in order to optimize overall performance while maintaining the highest level of safety. The desire to have these design tools and capabilities has also driven the continued development 
of computational fluid methods.

This chapter presents an overview of the fluid simulation method used in this research. In addition, several validation exercises verify that the method is capable of accurately simulating laminar, three-dimensional, chemically reacting hypersonic flows over a sharp-nose elliptic cone and a blunt-nose elliptic cone. Finally, the chapter summarizes the new features and capabilities added to the flow solver as a result of this thesis.

\subsection{LeMANS: An Overview}

Flow-field results are obtained using Computational Fluid Dynamics (CFD) to solve the Navier-Stokes equations. The CFD computations are executed using the Michigan Aerothermodynamic Navier-Stokes (LeMANS) code which was developed at the University of Michigan [56, 57, 58].

LeMANS is a general 2D/axisymmetric/3D, parallel, unstructured finite-volume CFD code. The numerical fluxes between cells are discretized using a modified Steger-Warming Flux Vector Splitting (FVS) scheme, except near shock waves. In these regions, the original Steger-Warming FVS scheme is used because it provides sufficient dissipation to accommodate the discontinuity [59].

LeMANS may be employed with any of three thermodynamic models: perfect gas, equilibrium thermochemistry, and nonequilibrium. LeMANS employs a twotemperature or three-temperature model to account for thermal nonequilibrium and a standard finite-rate chemistry model to account for nonequilibrium chemistry. The two temperature model assumes that a single temperature, T, accounts for the translational and rotational energy modes of all species while the vibrational and electronic energy modes are accounted for by a separate temperature, $\mathrm{T}_{v e}$. In the three- 
temperature model, the rotational energy mode is separated from the translational energy mode [60, 61]. This feature is useful when investigating flows where thermal nonequilibrium may exist between the translational and rotational energy modes [62], but is not employed in this thesis because energy transfer between the translational and rotational modes is sufficiently fast in the flows of interest in this work.

The simulations are performed using second-order accurate spatial discretization and carrying double precision arithmetic throughout. LeMANS produces steadystate flow-field solutions using a first-order accurate time advancement scheme, thus small time steps and an appropriate CFL number ensure accuracy [63]. Time advancement is performed using either explicit, point implicit, or a line implicit advancement. The line implicit advancement improves the layout of the sparse linear system of equations by arranging the equations into a near-tridiagonal form which greatly reduces the computational cost associated with solving the system of equations [64].

For a single temperature (local thermodynamic equilibrium) model with finite rate chemistry, the conservation equations are:

$$
\begin{gathered}
\frac{\partial \rho_{s}}{\partial t}+\nabla \cdot\left(\rho_{s} \mathbf{u}+\mathbf{J}_{s}\right)=\dot{\omega}_{s} \\
\frac{\partial \rho \mathbf{u}}{\partial t}+\nabla \cdot(\rho \mathbf{u} \mathbf{u}+p \mathbf{I}-\tau)=0 \\
\frac{\partial E}{\partial t}+\nabla \cdot\left((E+p) \mathbf{u}-\tau \cdot \mathbf{u}+\mathbf{q}+\Sigma\left(\mathbf{J}_{s} h_{s}\right)\right)=0
\end{gathered}
$$

where $\rho_{s}$ is the density of species, $s$, and $\mathbf{u}$ is the mass averaged bulk velocity vector. The species diffusion flux, $\mathbf{J}_{s}$, species enthalpy, $h_{s}$, and species mass production rate, 
$\dot{\omega}_{s}$, represent transport and chemical reactions for each species, s. The conservation of momentum, Eqn. 2.2, contains the total density, $\rho$, the pressure, $p$, the identity matrix, I, and a $3 \times 3$ tensor containing all the products of the components of the velocity vector, as seen in Eqn. 2.4.

$$
\mathbf{u} \mathbf{u}=\left[\begin{array}{ccc}
u^{2} & u v & u w \\
v u & v^{2} & v w \\
w u & w y & w^{2}
\end{array}\right]
$$

LeMANS assumes the fluid is continuous and Newtonian. It also assumes Stokes' hypothesis [65], to determine the viscous stresses, $\tau$ :

$$
\tau_{i j}=\mu\left(\frac{\partial u_{j}}{\partial x_{i}}+\frac{\partial u_{i}}{\partial x_{j}}\right)-\frac{2}{3} \mu \nabla \cdot \mathbf{u} \delta_{i j}
$$

where $\mu$ is the mixture coefficient of viscosity, which is determined using Wilkes semi-empirical mixing rule [66]. The delta operator, $\delta_{i j}$, equals one when $i=j$ and zero elsewise.

The conservation of energy equation, Eqn. 2.3, specifies the total energy per unit volume of the gas mixture, $E$, and $\mathbf{q}$ is the total heat flux vector. For the two temperature cases (thermodynamic nonequilibrium), the vibrational-electronelectronic energy equation is also solved:

$$
\frac{\partial E_{v e}}{\partial t}+\nabla \cdot\left(\left(E_{v e}\right) \mathbf{u}+\mathbf{q}_{v e}+\Sigma\left(\mathbf{J}_{s} e_{v e, s}\right)\right)=\dot{\omega}_{v e}
$$

where $E_{v e}$ is the vibrational-electron-electronic energy per unit volume of the gas mixture, $\mathbf{q}_{v e}$ is the vibrational-electron-electronic heat flux vector, and $e_{v e, s}$ is the species vibrational-electron-electronic energy per unit mass. The vibrational energy 
source term, $\dot{\omega}_{v e}$, is an approximation to the rate of vibrational-electronic work done by the production and destruction of species due to chemical reactions, energy transfer between nonequilibrium modes, and work done on electrons by the electric field induced by the electron pressure gradient [56].

The heat fluxes are modeled using Fourier's law which uses a mixture thermal conductivity for each energy mode. The species mass diffusion flux, $\mathbf{J}_{s}$, is determined using a modified form of Fick's law that enforces the restriction that the sum of the diffusion fluxes is zero and that the plasma maintains charge neutrality when the ionized species are present in the flow. A harmonic oscillator is used to model the species vibrational energy per unit mass, $e_{v e, s}$. Full details of the thermodynamic properties, transport coefficients, and finite rate chemistry models are available in [67].

Parallelization of the solver is implemented using Message Passing Interface (MPI) subroutine calls. The domain is divided among any number of processors using METIS [68], which partitions the domain to minimize boundary lengths, while balancing the numbers of volumetric cells per partition. Thermal equilibrium and a five species finite rate air chemistry model $\left(\mathrm{N}_{2}, \mathrm{O}_{2}, \mathrm{NO}, \mathrm{N}\right.$, and $\left.\mathrm{O}\right)$, based on Park's 1990 chemistry formulation [69] are used in the simulations presented throughout this thesis, unless otherwise stated. In addition, line implicit time advancement is used to minimize the computational cost needed to achieve a converged solution for all simulations preformed.

\subsection{LeMANS: Validation}

Although the solver, LeMANS, is an established two-dimensional, axisymmetric, and three-dimensional code [67], additional validation exercises are warranted 
to demonstrate its accuracy for scenarios of interest to this thesis, namely hypersonic, laminar, chemically reacting and non-reacting flows over bodies with sharp or blunt three-dimensional profiles. The following subsections describe the experimental setup and computational validation results for hypersonic flows over a sharp-nose elliptic cone and a blunt-nose elliptic cone. The blunt-nose elliptic cone geometry is of particular importance because it is used in subsequent chapters as a representative hypersonic geometry to quantify changes made to the flow-field due to the introduction of plasma-based flow control devices.

\subsubsection{Sharp-Nose Elliptic Cone}

Three-dimensional calculations are carried out for a Mach 7.93 sharp-nose elliptic cone originally studied experimentally by Kimmel et al. [70, 71]. Mounted parallel to the freestream, the elliptic cone geometry consists of a 2:1 aspect ratio with a half angle along the major axis of $14^{\circ}$ and a total length $L=1.016 \mathrm{~m}$. It is machined from stainless steel with a $40 \mu \mathrm{m}$ nose radius and surface roughness less than 0.81 $\mu \mathrm{m}$. The flow conditions are listed in Table 2.1.

A structured grid is employed because it is known to produce better results than unstructured meshes in regions near the surface of the body and through a shock [72]. One quarter of the geometry is simulated because planes of symmetry exist along the major and minor axes. The $40 \mu \mathrm{m}$ nose radius is accounted for along the tip's minor axis, resulting in an $80 \mu \mathrm{m}$ radius along the major axis because of the elliptical geometry. The spherical nose tip region is blended with the elliptical geometry by requiring the second derivative of the surface shape to be zero.

The model is aligned with the $x$-axis in the axial direction, the $y$-axis in the horizontal direction, and the $z$-axis in the vertical direction. A cylindrical coordinate 
Table 2.1: Flow conditions for the sharp-nose elliptic cone experiment conducted by Kimmel et al. [70, 71].

\begin{tabular}{crl}
\hline \hline Parameter & Value \\
\hline $\mathrm{M}$ & 7.93 & \\
$u_{\infty}$ & $1180.0 \mathrm{~m} / \mathrm{s}$ \\
$\mathrm{T}_{\infty}$ & 54.6 & $\mathrm{~K}$ \\
$\mathrm{~T}_{w}$ & $303.0 \mathrm{~K}$ \\
$\mathrm{~T}_{0}$ & $728.0 \mathrm{~K}$ \\
$p_{\infty}$ & 165.0 & $\mathrm{~Pa}$ \\
$\rho_{\infty}$ & 0.011 & $\mathrm{~kg} / \mathrm{m}^{3}$ \\
$\mu_{\infty}$ & $3.77 \times 10^{-6}$ & $\mathrm{~kg} / \mathrm{m} \cdot \mathrm{s}$ \\
$\operatorname{Re}_{x}$ & $3.44 \times 10^{6}$ & $\mathrm{~m}$ \\
$\operatorname{Re}_{L}$ & $3.50 \times 10^{6}$ & \\
\hline \hline
\end{tabular}

system is also employed with $\theta=0^{\circ}$ at the top centerline of the model ( $z$-axis) and $\theta=90^{\circ}$ at the leading edge (y-axis) as seen in Fig. 2.1.

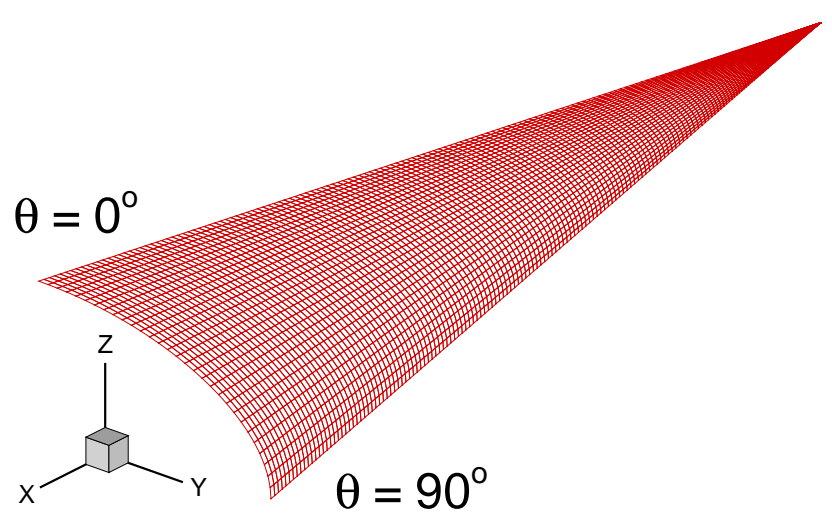

Figure 2.1: Surface of the sharp-nose elliptic cone grid with both Cartesian and cylindrical coordinate systems. $(330 \times 40 \times 30)$

A gradual increase in grid spacing is used along the conic body with the smallest spacing near the tip. Radial points are algebraically spaced to increase the number of points close to the body. Grid points are equally spaced along the circumference. 
As a result, cell clustering occurs near the surface and the tip of the body. A grid independence study is conducted with $i \times j \times k$ grid dimensions changing from $330 \times 40 \times 30($ coarse $)$, to $440 \times 50 \times 40($ medium $)$, to $550 \times 60 \times 50$ (fine) .

Figure 2.2 shows the density contours for the flow-field along the symmetry planes and various axial locations, which closely match computational results by Gosse and Kimmel [73].

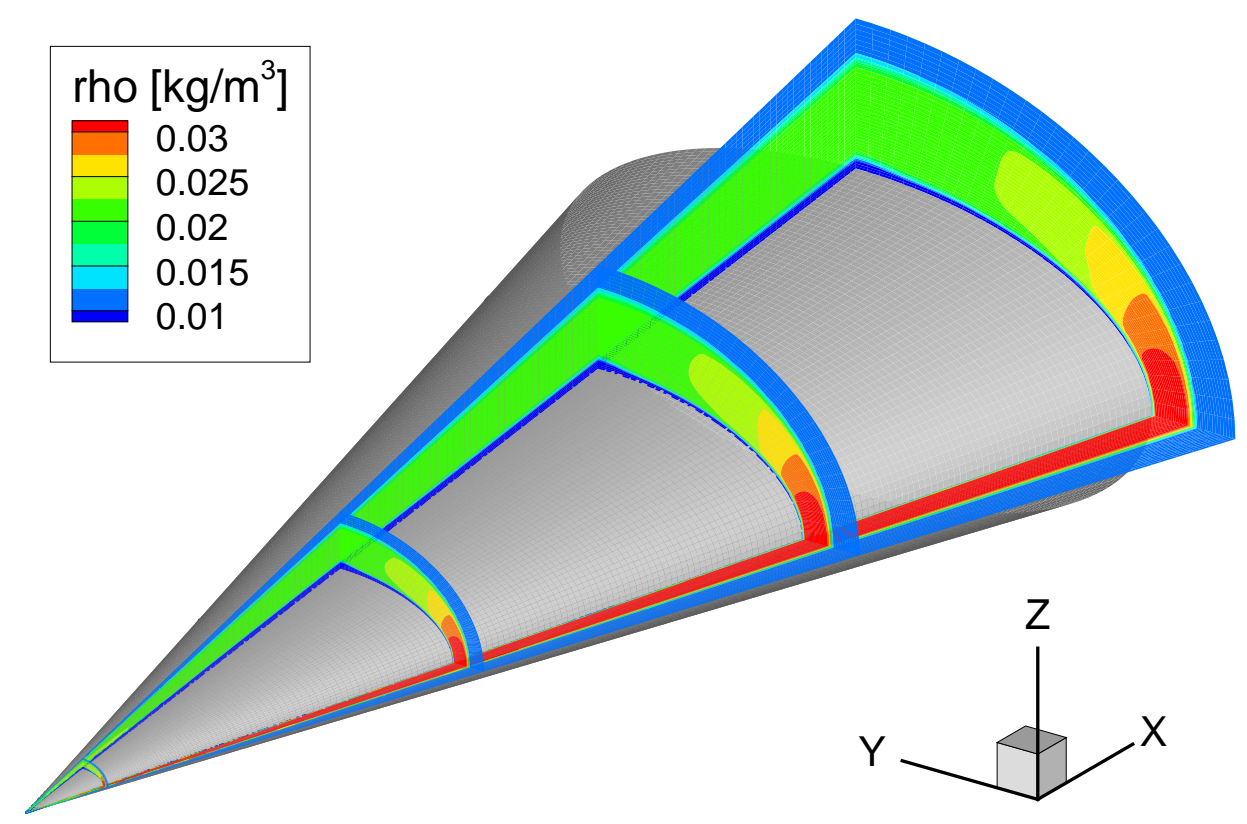

Figure 2.2: Density contours for Mach 7.93 air flow around a sharp-nose elliptic cone. $(330 \times 40 \times 30)$

The 2:1 aspect ratio of the elliptic geometry results in a circumferential pressure gradient which generates a circumferential velocity component. This spanwise velocity component results in a highly three-dimensional flow-field as the flow rolls up along the top centerline, thus increasing the boundary layer thickness in that region. The three-dimensionality of the flow-field warrants the investigation of the pressure coefficient, $\mathrm{Cp}$, and the Stanton number, St, at several axial slices and along various rays along the body to ensure a grid-independent solution is achieved: 


$$
\begin{gathered}
\mathrm{Cp}=\frac{p_{\mathrm{w}}-p_{\infty}}{1 / 2 \rho_{\infty} u_{\infty}{ }^{2}} \\
\mathrm{St}=\frac{\mathrm{q}_{\mathrm{w}}}{\rho_{\infty} u_{\infty}\left(h_{0}-h_{\mathrm{w}}\right)}
\end{gathered}
$$

where $p_{\infty}, \rho_{\infty}$ and $u_{\infty}$ are the freestream pressure, density and velocity, $p_{\mathrm{w}}$ is the surface (wall) pressure, $\mathrm{q}_{\mathrm{w}}$ is the heat flux to the wall, and $h_{0}$ and $h_{\mathrm{w}}$ are the stagnation and wall enthalpies, respectively. Figure 2.3 shows very little change in Cp or St between the 'medium' and 'fine' grids. Therefore, grid independence is considered achieved with the 'medium' grid, and is used in the rest of the analysis.

Cross-sectional slices are extracted to match the locations of the pressure and heat transfer measurements made by Kimmel et al. [71]. Figure 2.4(a) shows the nondimensional pressure along the circumference of the body at $x / L=0.625$. The pressure is relatively constant from the top centerline $\left(\theta=0^{\circ}\right)$, to the shoulder $\left(\theta=45^{\circ}\right)$, but then exhibits a noticeable increase between the shoulder and the leading edge $\left(\theta=90^{\circ}\right)$. Kimmel et al. also provide computational results obtained from a Parabolized Navier-Stokes (PNS) code [70, 71], which are included in the figures as an additional reference.

Although the cone is sharp, the formation of the boundary layer at its tip results in a noticeable rise in pressure and temperature near the stagnation point, which can be seen in Fig. 2.4(b) for two different rays. The pressure quickly relaxes as the flow proceeds along the rest of the cone due to the viscous interaction. The PNS solution does not capture this behavior within the stagnation region because of the physical simplifications inherent to the PNS formulation.

Even though the surface geometry roughness is less than $0.81 \mu \mathrm{m}$, the large unit Reynolds number $(\operatorname{Re} / x)$, noisy wind tunnel conditions, and total length of the 


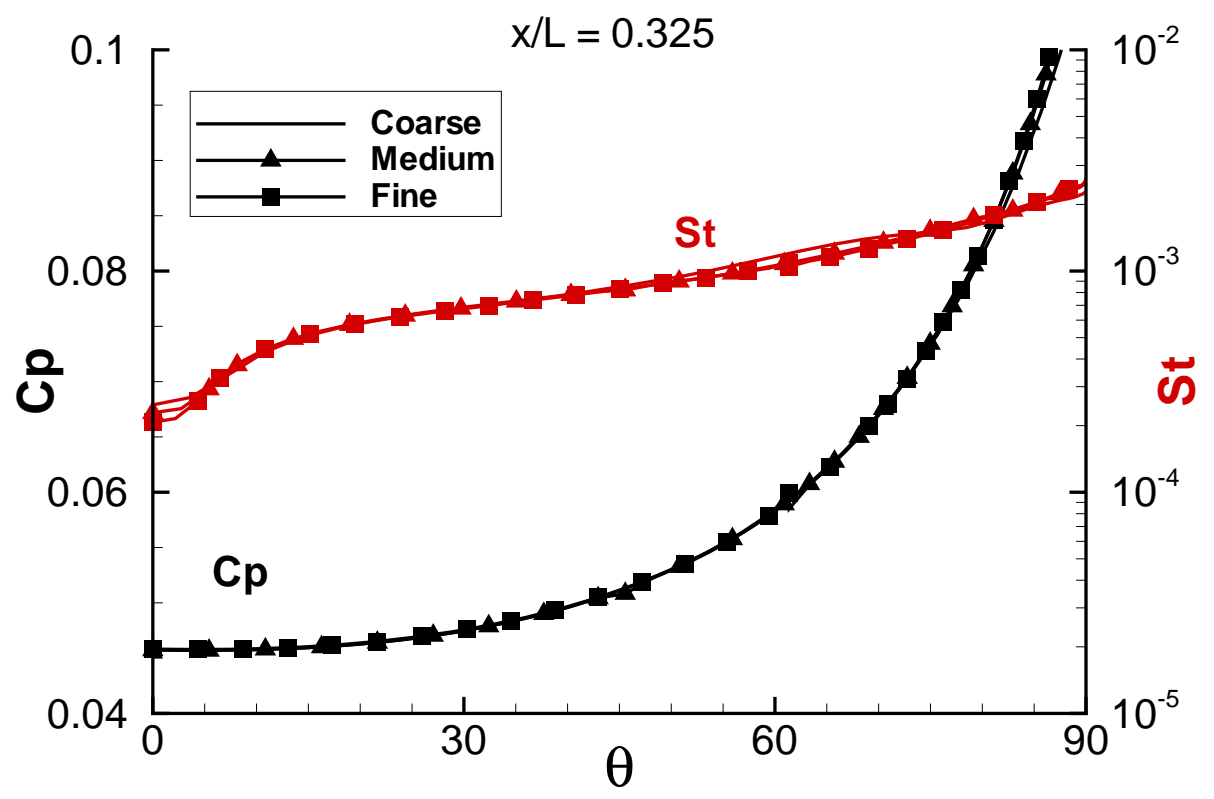

(a) Around the circumference at $x / L=0.325$.

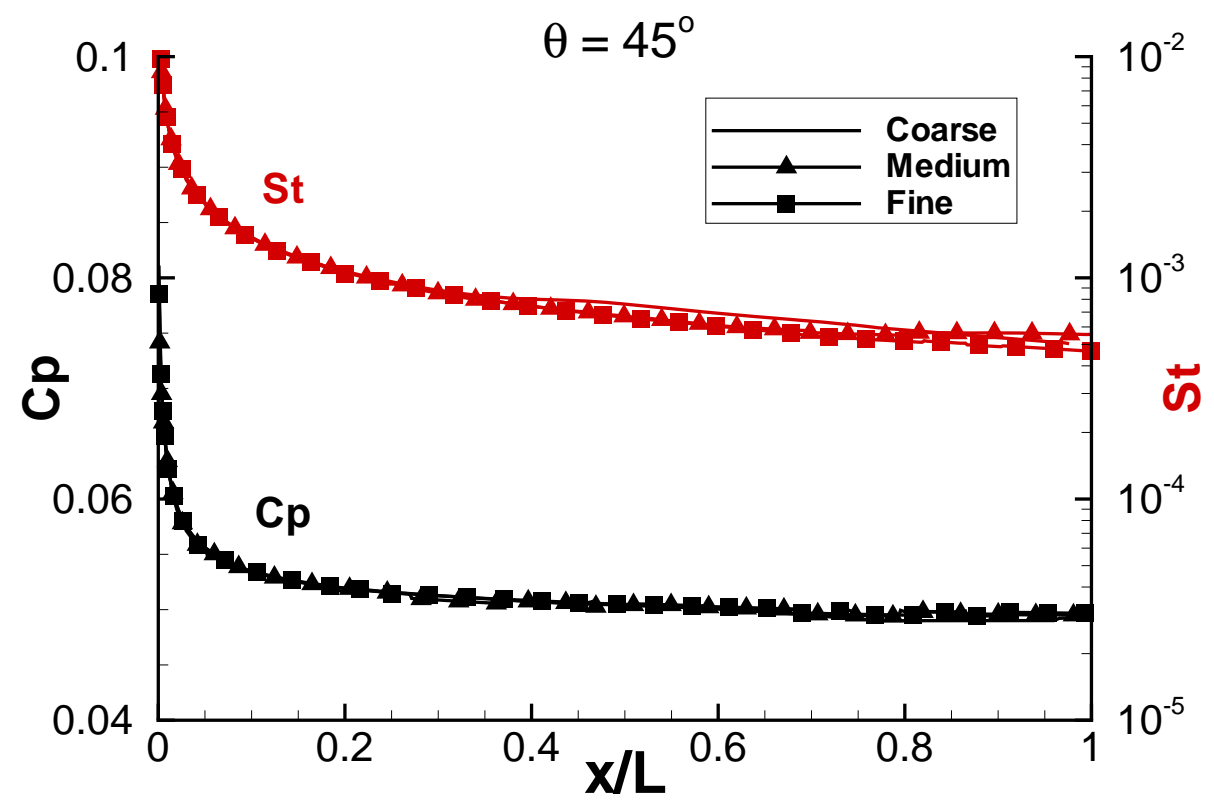

(b) Along the $\theta=45^{\circ}$ ray.

Figure 2.3: Grid independence study for Mach 7.93 air flow around a sharp-nose elliptic cone $(L=1.016 \mathrm{~m})$. 


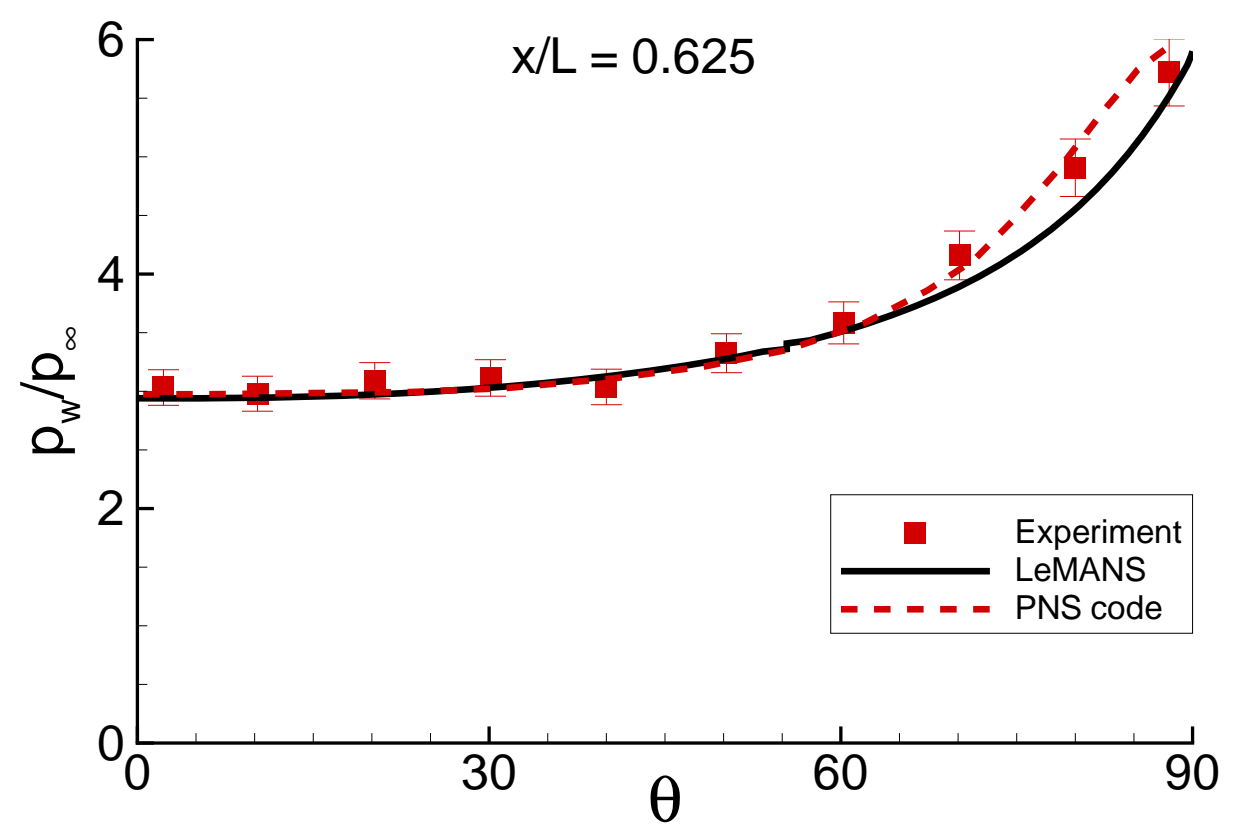

(a) Pressure around the circumference.

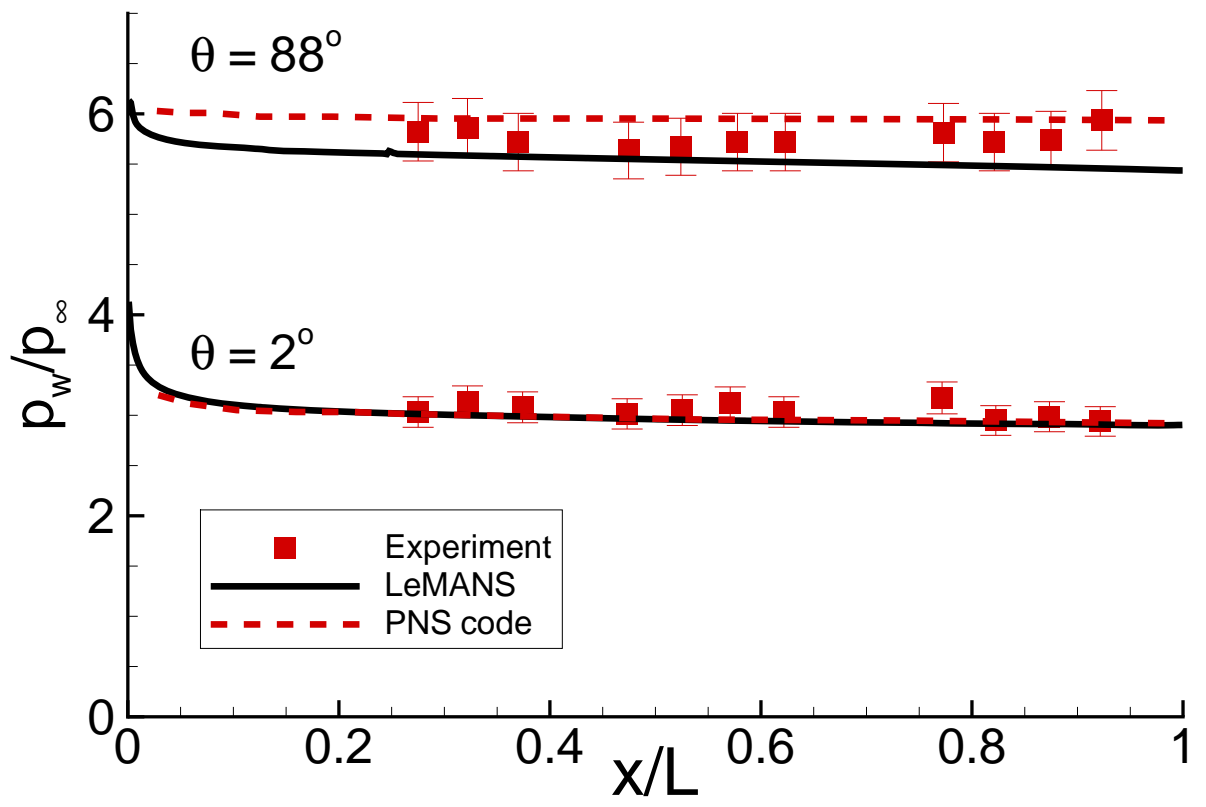

(b) Pressure along rays.

Figure 2.4: Nondimensional pressure distributions for Mach 7.93 air flow around a sharp-nose elliptic cone ( \pm 5 percent experimental uncertainty). PNS calculations and experimental data from [71]. 
geometry cause the boundary layer to eventually transition to become turbulent as it proceeds along the body. Since LeMANS does not currently have a turbulence model, the numerical results in the transitional and turbulent regions should be disregarded. Plots of the Stanton number as a function of Reynolds number (where $x$ is the axial location along the geometry), are presented in Figs. 2.5(a), 2.5(b), and 2.5(c) for $\theta=0,45$, and $88^{\circ}$, respectively. As seen in all three plots, a laminar flow develops along the forebody and then transitions to turbulent as it proceeds. The data from [71] are for a unit Reynolds number of: $\operatorname{Re} / x=1.7 \times 10^{6} \mathrm{~m}^{-1}$ and $6.6 \times 10^{6} \mathrm{~m}^{-1}$, whereas this scenario has: $\operatorname{Re} / x=3.4 \times 10^{6} \mathrm{~m}^{-1}$. However, because of flow similarity, the unit Reynolds number does not affect the Stanton number in the laminar region, and the flow solver accurately predicts the distribution within the laminar zone.

\subsubsection{Blunt-Nose Elliptic Cone}

A three-dimensional validation study is also performed on a Mach 14.2 bluntnose elliptic cone studied experimentally by Nowlan et al. [74] The model is mounted parallel to the freestream and has a 2:1 aspect ratio, a half angle along the major axis of $10^{\circ}$, and a length $L=0.21 \mathrm{~m}$. Details of the geometry are provided in Fig. 2.6. The flow conditions are listed in Table 2.2 .

A structured grid is generated following the same procedures and coordinate system as the sharp-nose elliptic cone described in Section 2.2.1. A grid independence study is conducted with $i \times j \times k$ grid dimensions changing from $150 \times 30 \times 30$ (coarse), to $300 \times 60 \times 60($ medium $)$, to $380 \times 80 \times 80$ (fine).

Because the wall enthalpy is not immediately computed by the flow solver, the nondimensional heat flux is defined in terms of the freestream kinetic energy flux, as seen in Eqn. 2.9: 


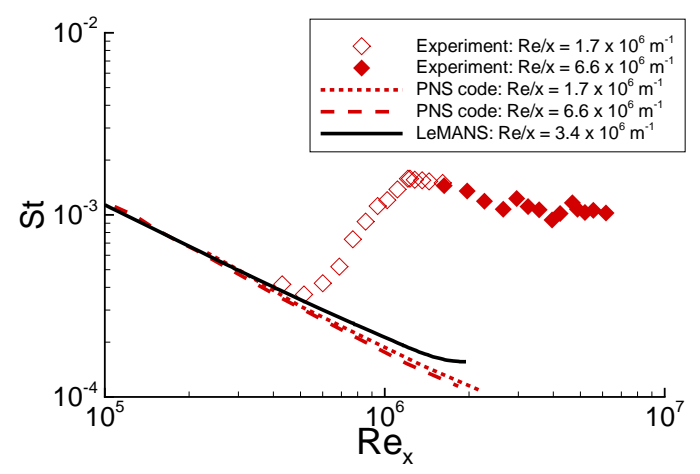

(a) Top centerline, $\theta=0^{\circ}$

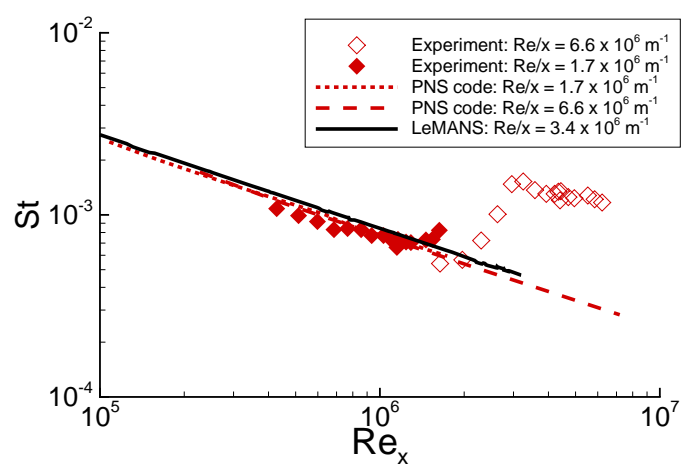

(b) Shoulder, $\theta=45^{\circ}$

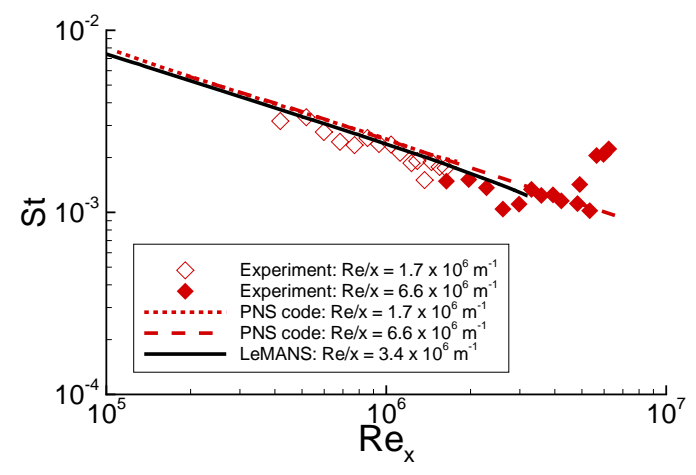

(c) Leading edge, $\theta=88^{\circ}$

Figure 2.5: Stanton number distributions for Mach 7.93 air flow around a sharp-nose elliptic cone (symbol size reflects \pm 10 percent experimental uncertainty). PNS calculations and experimental data from [71].
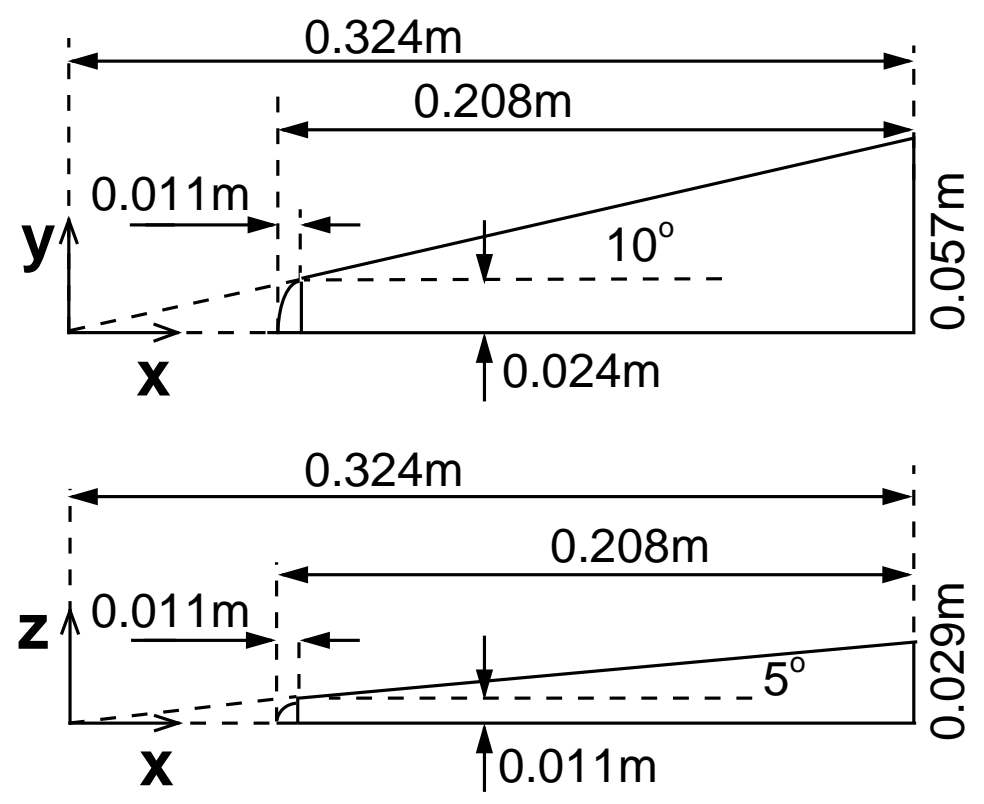

Figure 2.6: Blunt-nose elliptic cone geometry. Adapted from [74]. 
Table 2.2: Flow conditions from Run 15 of the blunt-nose elliptic cone experiment conducted by Nowlan et al. [74].

\begin{tabular}{crl}
\hline \hline Parameter & \multicolumn{2}{c}{ Value } \\
\hline Mach & 14.2 & \\
$u_{\infty}$ & 2190 & $\mathrm{~m} / \mathrm{s}$ \\
$\mathrm{T}_{\infty}$ & 59.3 & $\mathrm{~K}$ \\
$\mathrm{~T}_{w}$ & 294.0 & $\mathrm{~K}$ \\
$\mathrm{~T}_{0}$ & 211.0 & $\mathrm{~K}$ \\
$p_{\infty}$ & 51.0 & $\mathrm{~Pa}$ \\
$\rho_{\infty}$ & 0.003 & $\mathrm{~kg} / \mathrm{m}^{3}$ \\
$\mu_{\infty}$ & $4.3 \times 10^{-6}$ & $\mathrm{~kg} / \mathrm{m} \cdot \mathrm{s}$ \\
$\operatorname{Re} / x$ & $1.53 \times 10^{6}$ & $\mathrm{~m}-1$ \\
$\operatorname{Re} L$ & $3.17 \times 10^{5}$ & \\
\hline \hline
\end{tabular}

$$
\mathrm{C}_{\mathrm{h}}=\frac{q_{\mathrm{w}}}{1 / 2 \rho_{\infty} u_{\infty}^{3}}
$$

where $q_{\mathrm{w}}$ is the heat flux to the wall, and $\rho_{\infty}$ and $u_{\infty}$ are the freestream gas density and velocity. The heat flux to the wall is the summation of both the translationalrotational and vibrational-electronic heat fluxes, $q_{\mathrm{w}}=q_{\mathrm{w} t r}+q_{\mathrm{w} v e}$, when thermal nonequilibrium is assumed in the simulations. Figure 2.7 shows very little change in $\mathrm{Cp}$ or St between the 'medium' and 'fine' grids; therefore the 'medium' grid is considered grid-independent and used in the rest of the analysis. (Recall Cp is defined in Eqn. 2.7.)

The flow-field is computed using a standard five species finite rate air chemistry model $\left(\mathrm{N}_{2}, \mathrm{O}_{2}, \mathrm{NO}, \mathrm{N}\right.$, and $\left.\mathrm{O}\right)$, based on Park's 1990 chemistry formulation [69]. The 2:1 aspect ratio creates a circumferential pressure gradient between the top centerline and leading edge symmetry planes. The pressure gradient is, however, less 


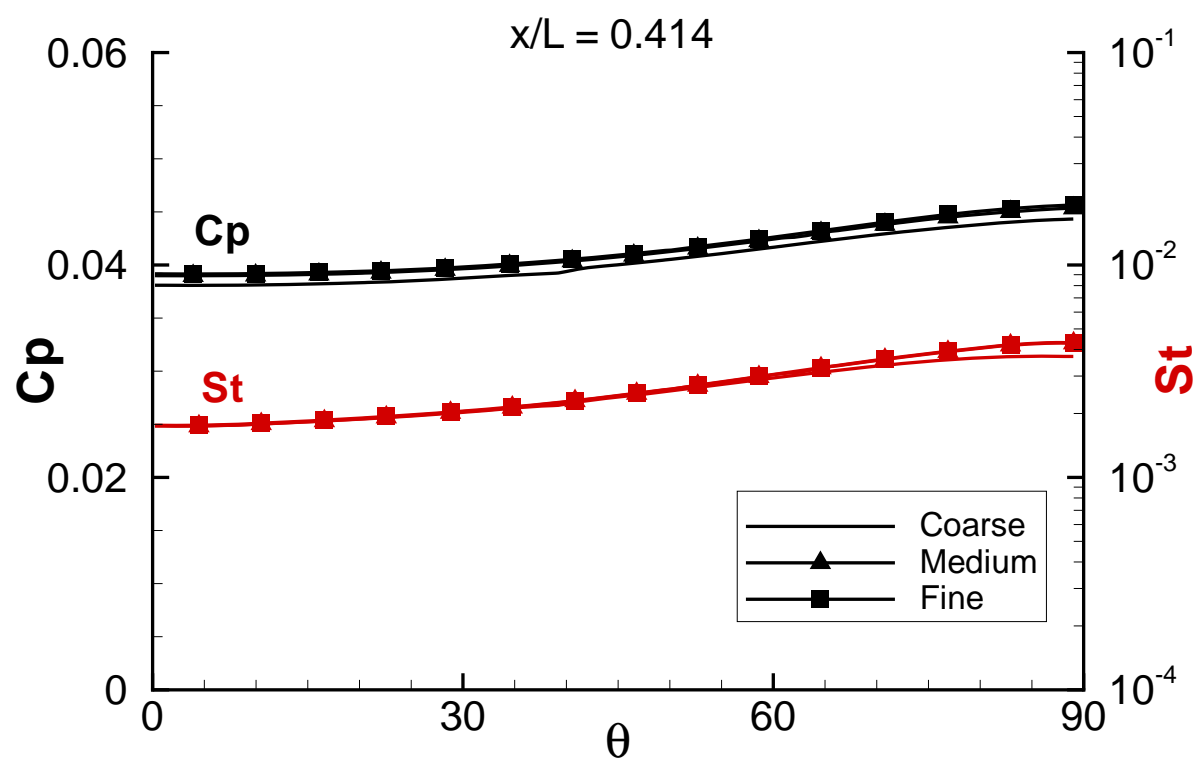

(a) Around the circumference.

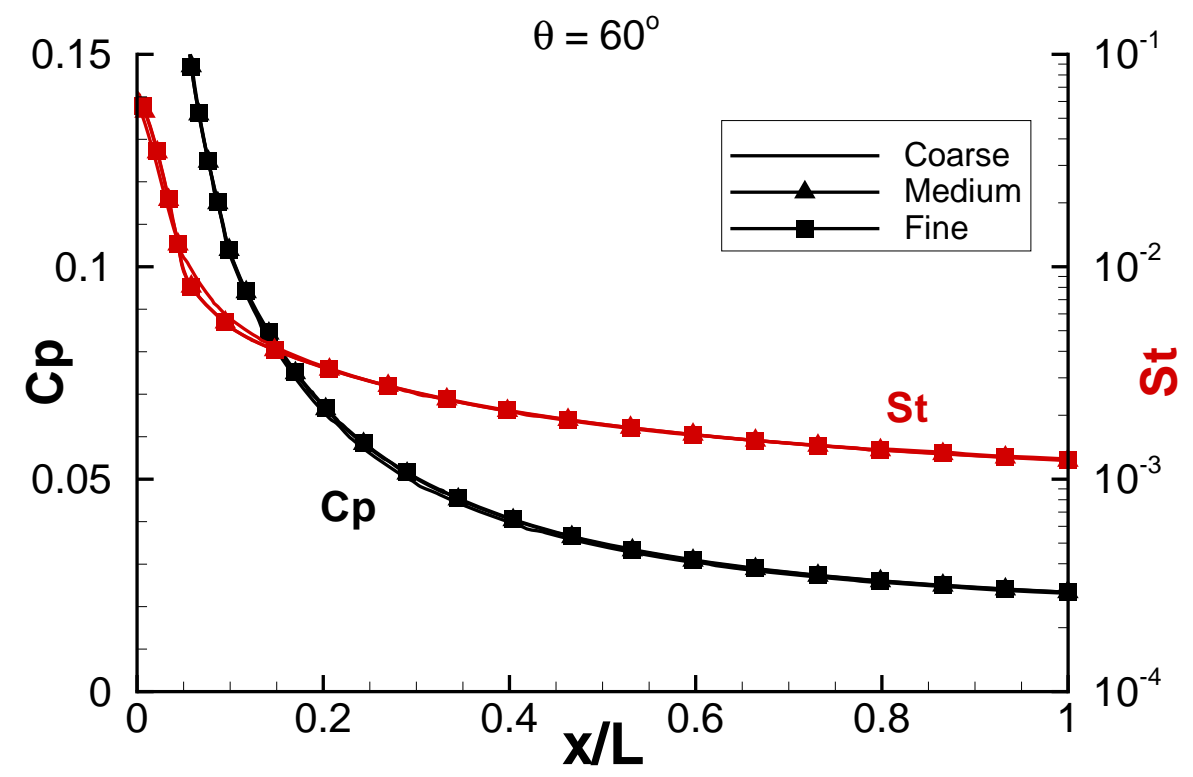

(b) Along rays.

Figure 2.7: Grid independence study for Mach 14.2 air flow around a blunt-nose elliptic cone $(L=0.21 \mathrm{~m})$. 
distinguishable than the pressure gradient observed in the sharp-nose elliptic cone scenario (Fig. 2.3), because the blunt geometry produces a strong bow shock, instead of an attached oblique shock, which dramatically increases the pressure in the stagnation region. The high pressure in the stagnation region provides the downstream flow with a more uniform pressure distribution along the circumference of the cone, as seen in Fig. 2.8

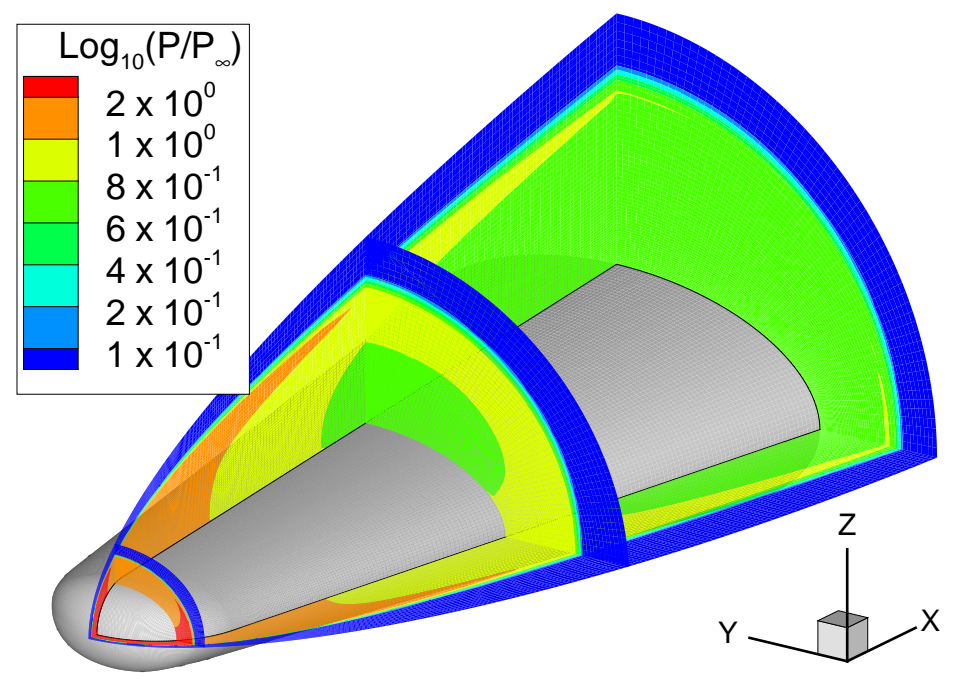

Figure 2.8: Pressure contours for Mach 14.2 air flow around a blunt-nose elliptic cone.

Following a trend similar to the sharp cone validation exercise, the pressure coefficient is relatively constant from the top centerline to the shoulder, followed by a gradual rise between the shoulder and the leading edge, as seen in Fig. 2.9(a). The behavior is also observed by Atkinson et al. in [75], although their results show a much larger pressure rise, especially near the leading edge.

Compared to the sharp cone simulations in Fig. 2.4(b), the pressure coefficient distribution along the rays in Fig. 2.9(b) experiences a significant increase near the stagnation stagnation point because of the strong bow shock. Stanton number distributions in Fig. 2.10 show the profiles obtained with LeMANS follow the same 
general trends as the experimental measurements.

Overpredictions observed in the pressure coefficient and Stanton number distributions may be due to several influences not accounted for in the simulations. Heat transfer measurements were made using 'thin-film' heat-transfer gauges located on the geometry surface, which was constructed out of brass. The heat transfer rates are derived from the instantaneous temperature measurements using a first-order approximation and do not account for any influence the surrounding brass geometry may have on the 'thin-film' temperature. These assumptions suggest that the reported experimental uncertainty of \pm 4.5 percent may only correspond to the uncertainty in the actual instantaneous temperature measurements collected and not the experimental uncertainty of the heat transfer rate as reported.

Nowlan et al. noted an uncertainty of \pm 7 percent in the freestream flow conditions and a very cold freestream flow $\left(\mathrm{T}_{\infty}=59.3 \mathrm{~K}\right)$, which could have lead to condensation in the nozzle. In addition, the CAL 48-inch shock tunnel could have developed 'frozen' freestream conditions $\left(\mathrm{T}_{v e} \gg \mathrm{T}_{\infty}\right)$, as the flow accelerated through the nozzle. Nompelis et al. demonstrated, computationally, that accounting for vibrational nonequilibrium freestream conditions greatly improved agreement between computational and experiment heat transfer measurements collected for a hypersonic double-cone experiment [76]. This experiment was conducted at the same facility as these blunt-nose elliptic cone experimental measurements, but used a different shock tunnel [77]. Despite the discrepancies, overall, LeMANS, effectively demonstrates its capability of accurately computing three-dimensional hypersonic laminar flows. 


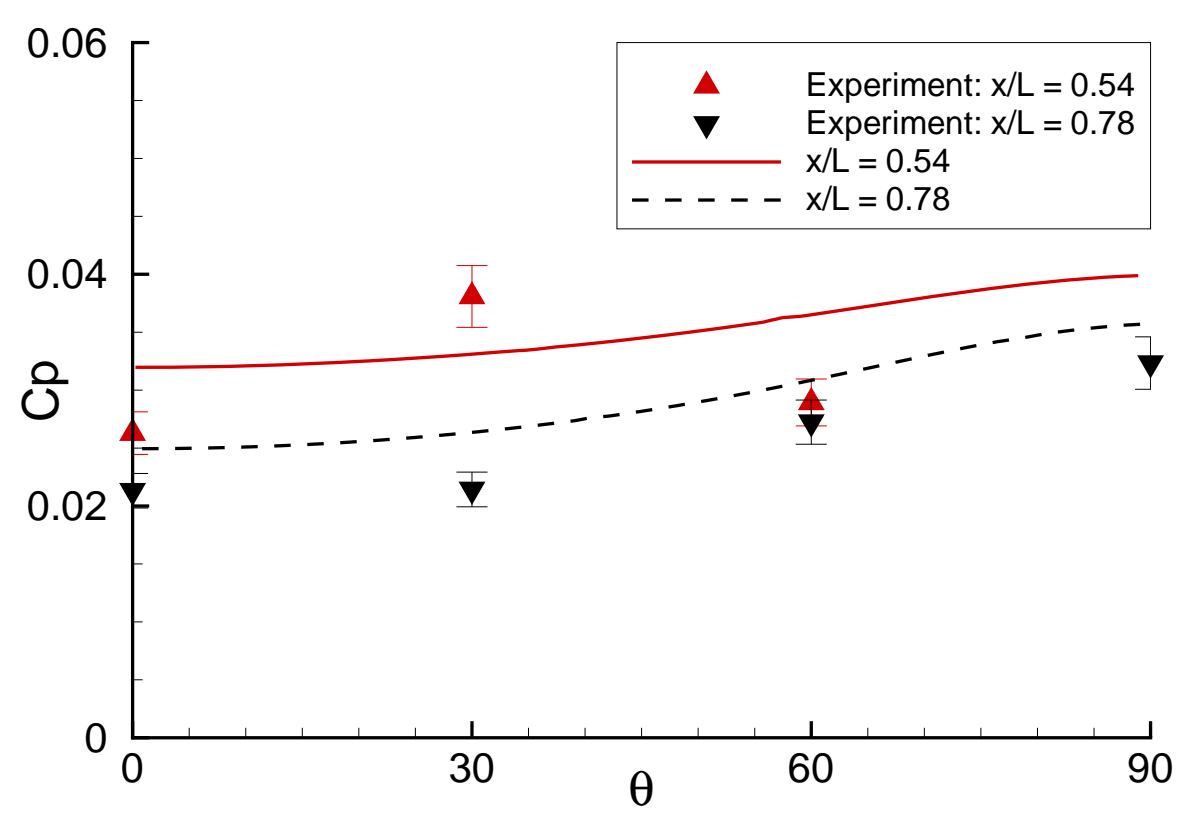

(a) Around the circumference

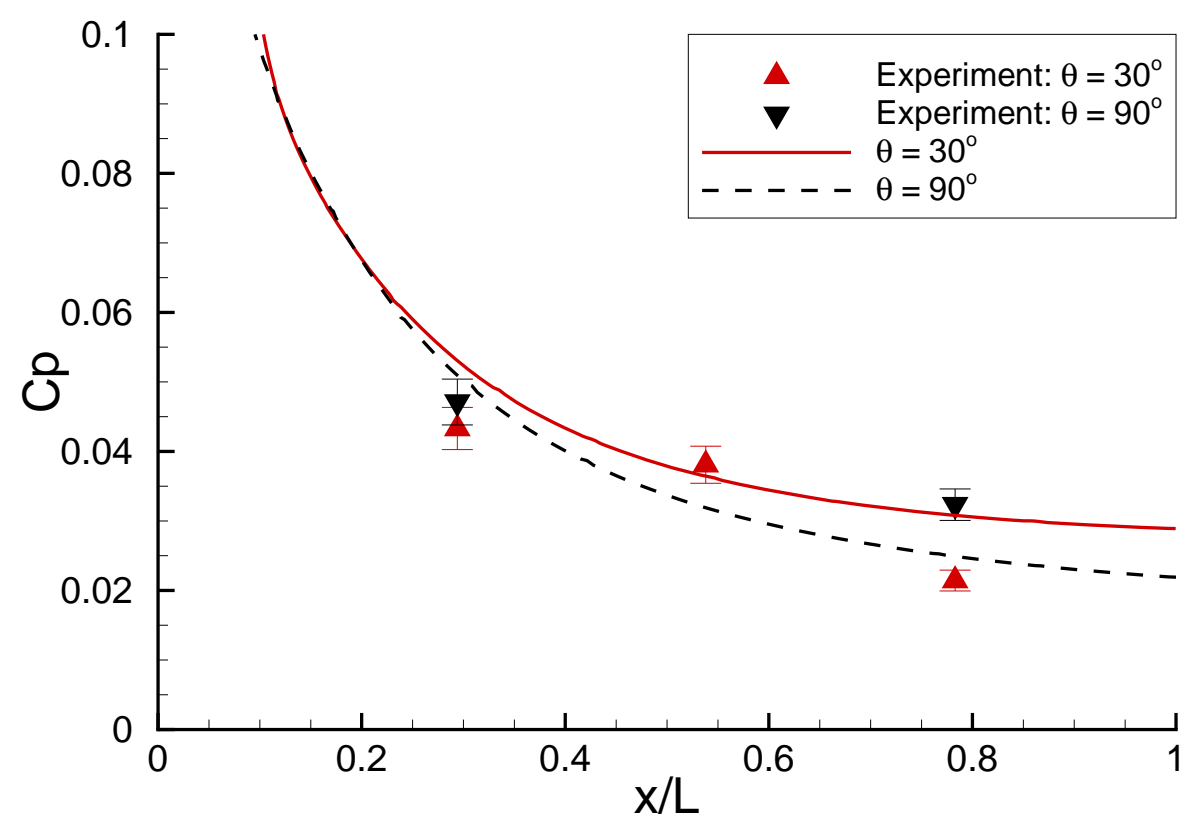

(b) Along rays

Figure 2.9: Pressure coefficient distributions for Mach 14.2 air flow around a bluntnose elliptic cone ( \pm 7 percent experimental uncertainty). Experimental data from Ref. [74]. 


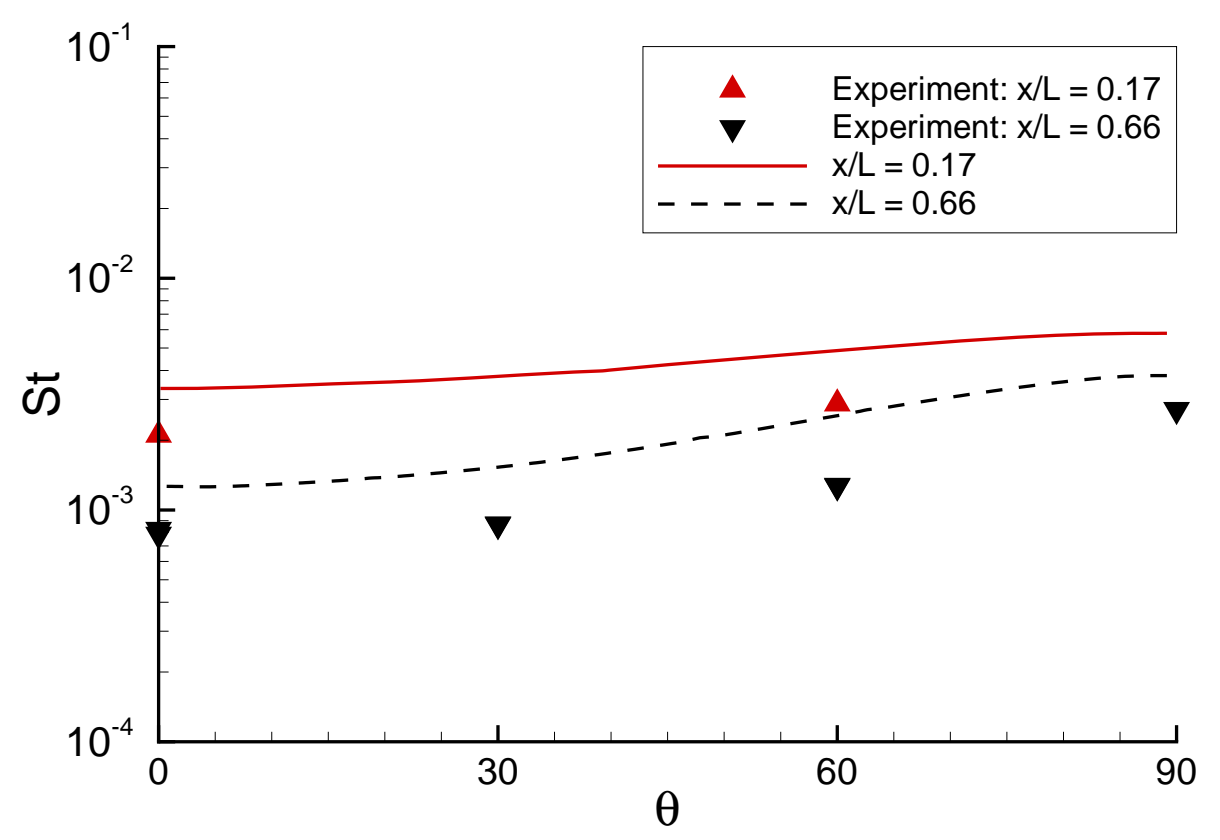

(a) Stanton number around the circumference

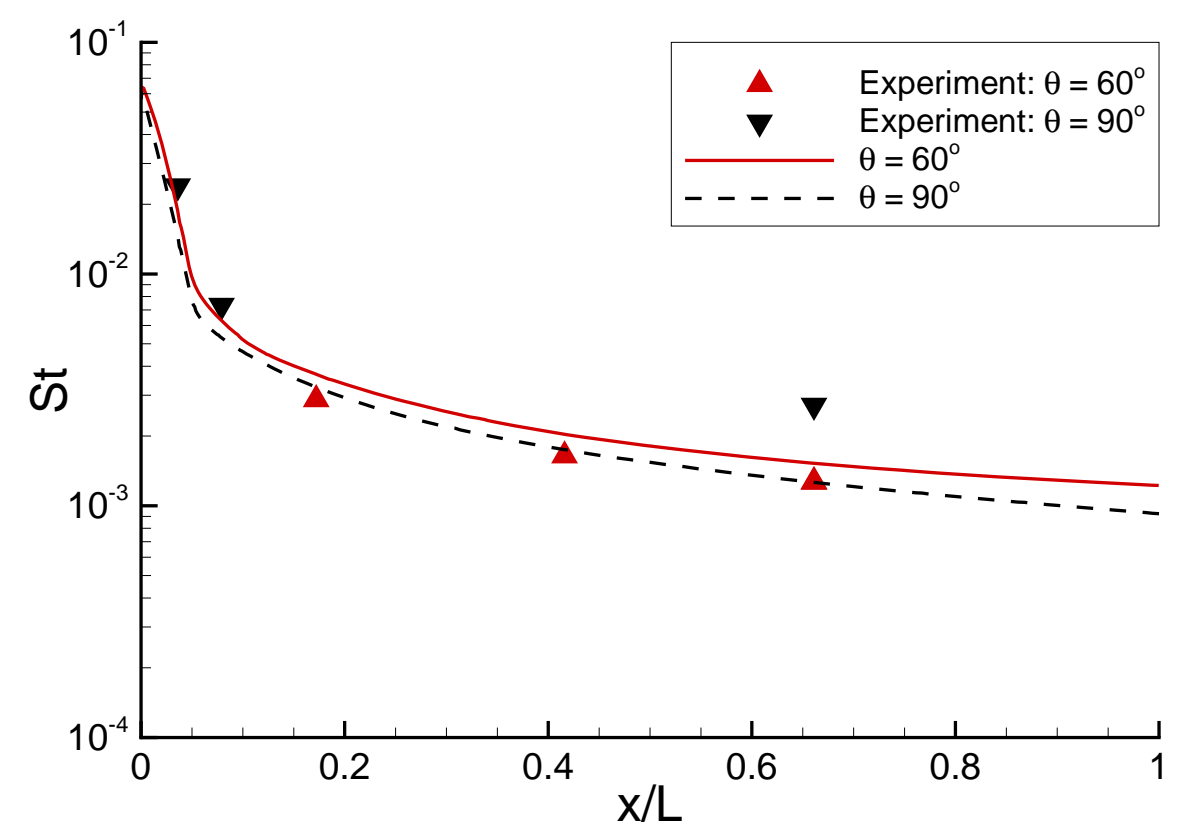

(b) Stanton number along rays

Figure 2.10: Stanton number distributions for Mach 14.2 air flow around a bluntnose elliptic cone (symbol size reflects \pm 4.5 percent experimental uncertainty). Experimental data from Ref. [74]. 


\subsection{LeMANS: New Features}

This section summarizes the new capabilities added to the flow solver as part of this thesis. These improvements are developed to extend the usefulness of the flow solver as it is applied to simulate flow-fields beyond its original scope. Two of the routines developed allow large meshes, which were previously beyond the capability of LeMANS, to be employed. The other routines allow for the investigation of the energy deposition and electromagnetic effects on the flow.

\subsubsection{Pre-LeMANS}

This program is run before using the flow solver to partition the domain into a specified number of smaller domains. The routine creates the 'lines' needed by the line implicit iterative routine used for time advancement within the solver, and generates the ghost cells and cell connectivity information needed to operate the flow solver in parallel computations. Pre-LeMANS is necessary when computing solutions for large meshes (greater than 1 million cells for processors with 1 Gigabyte (GB) Random Access Memory (RAM) or 2.5 million cells for processors with 2 GB RAM), as the flow solver's internal partitioning subroutine will not run for meshes greater than those specified due to memory restrictions. The program is also beneficial when conducting a parametric study that uses the same mesh and number of processors because the computational effort needed to partition the mesh is only required once.

The program is incorporated into the solver, and is activated as part of the user input file. If the partitions already exist, then the solver employs a new subroutine to load the 'partitioned' mesh file. A detailed explanation of the program is available in Appendix A. 


\subsubsection{Post-LeMANS}

This program is used after the solver finishes exporting its output files, but is only necessary for parallel computations. Each processor used for a parallel simulation generates an output file that represents a different region of the entire flow-field. Because the domain is partitioned amongst multiple processors, each processor has additional interior 'ghost' cells to share information between processors. These cells of duplicate information result in unwanted 'interior walls' when the output solutions are studied using the visualization program, Tecplot 360@ [78], as seen in Fig. 2.11.

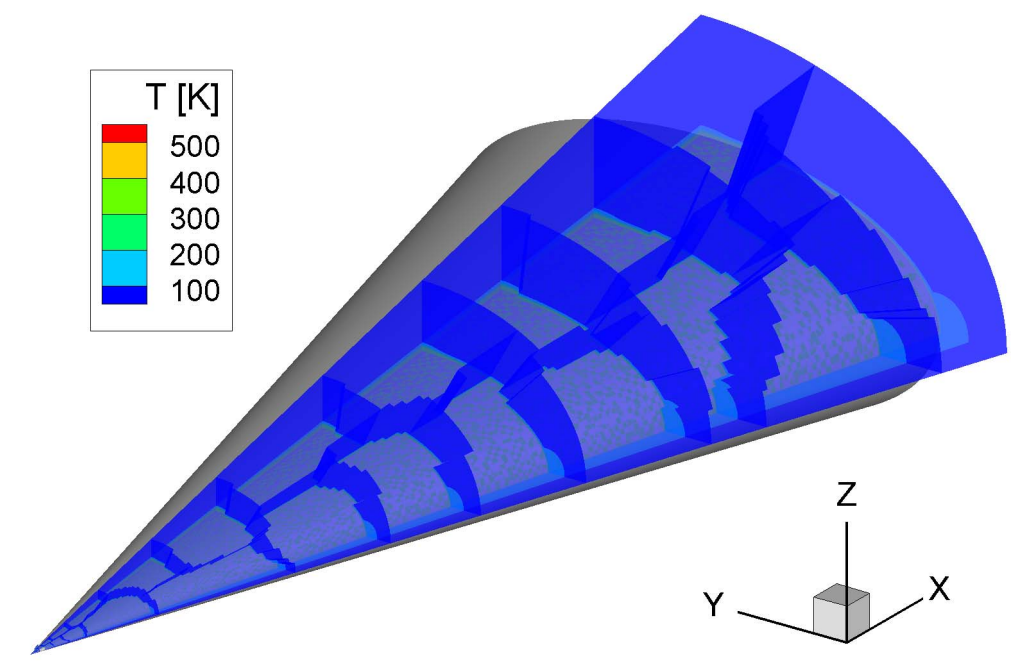

Figure 2.11: Temperature contours for Mach 7.93 air flow around a sharp-nose elliptic cone with the 'interior walls.' (before Post-LeMANS)

These 'interior walls' make it difficult to visually analyze the flow-field, and are a nuisance when trying to create clear images for presentations and publications. The Post-LeMANS program collects and combines these output files into a single file while removing duplicate interior 'ghost' cells and appropriately updating their adjoining cell connectivity information, as seen in Fig. 2.12.

The routine is particularly useful when many processors are used, a typical requirement for large computational domains. However, these large domains require 


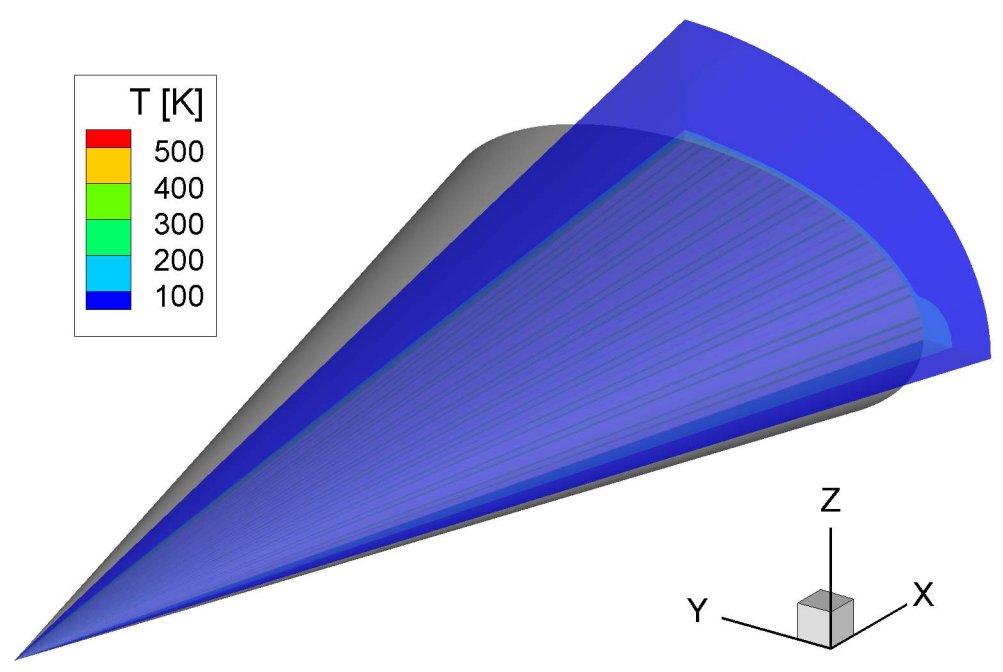

Figure 2.12: Temperature contours for Mach 7.93 air flow around a sharp-nose elliptic cone without the 'interior walls.' (after Post-LeMANS)

considerable computational effort to reorganize the resulting data. As a result, the Post-LeMANS utility is parallelized using Massage Passing Interface (MPI) calls to reduce the wall time necessary to combine the data. A detailed explanation of the program and its performance characteristics is available in Appendix B.

\subsubsection{Phenomenological Model of Volumetric Heating}

This feature directly inputs energy into specific locations in the domain. The shape and amount of energy input are defined by an exponentially decaying ellipsoid; the parameters are listed in a user input file. This capability is useful when investigating the downstream effects of a heating device. Full details of the model and its incorporation into the conservation equations are described in Section 3.2.

\subsubsection{LeMANS-MHD}

Electromagnetic effects on a flow can be important for hypersonic flows, especially when plasma-based devices are used to modify or control the flow. These effects are accurately accounted for in the conservation equations by the inclusion of 
the magnetic force in the conservation of momentum equation and total volumetric heating in the conservation of energy equation. The conservation of vibrationalelectron-electronic energy equation is also modified to allow for the corresponding heating of the vibrational modes through electron-molecule collisions. These additions to the standard conservation equations result in four new variables: $\gamma, \mathbf{j}, \mathbf{E}$, and $\mathbf{B}$, although the energy partitioning factor, $\gamma$, only needs to be defined for thermal nonequilibrium simulations, and can be estimated from external information.

A generalized form of Ohm's law is solved to determine the current density, $\mathbf{j}$, and electric field, E, while assuming the low magnetic Reynolds number approximation is valid. For low magnetic Reynolds numbers, the induced magnetic field is small compared to the applied magnetic field, so the magnetic field is not greatly affected by the fluid motion [50]. This means the magnetic field, $\mathbf{B}$, is imposed and must be specified by the user. Full details of the routine and its incorporation into the conservation equations are described in Chapter IV.

\subsubsection{Electrical Conductivity Models}

The current density, $\mathbf{j}$, which is derived in Section 4.2, is directly dependent on the gas electrical conductivity and is vital to accurately account for the electromagnetic effects in a flow. The electrical conductivity is typically determined using a semi-empirical formula valid for specific regimes of temperature, pressure, and gas composition. While these semi-empirical models are approximately valid and computationally inexpensive, they are not general, and can be problematic when flow conditions exceed the range of validity of the approximation. Nonetheless, several standard semi-empirical models are added to the solver and are accessible through input files. 
In addition to semi-empirical models, a separate routine is included that allows the user to develop a surrogate model of solutions to Boltzmann's equation for a specific gas composition. The Boltzmann solver provides a more widely applicable and physically accurate estimate of the electrical conductivity by using an extensive list of accurate collision cross-section data. The electrical conductivity data generated by the Boltzmann solver are used to develop a polynomial response surface model of the electrical conductivity that is accessible in LeMANS through an input file. Full details on the semi-empirical conductivity models and the surrogate model solutions of Boltzmann's equation are available in Chapter V.

\subsection{Summary}

This chapter outlined characteristics of the CFD code, LeMANS, which is used throughout this thesis. Validation exercises, involving the computational solutions of three-dimensional hypersonic, chemically-reacting flow around sharp-and blunt-nose elliptic cones verify that the code is capable of accurately simulating flow-fields in the flight regime this thesis explores. The chapter also summaries the new features added to the code, including routines that allow the user to simulate very large grids using many processors and then collate the resulting data into a single output file, facilitating the use of existing visualization programs. 


\section{CHAPTER III}

\section{Phenomenological Model of Volumetric Heating}

\subsection{Introduction}

Plasma actuators and various forms of volumetric energy deposition have received a good deal of research attention recently as a means of hypersonic flight control $[23,22]$. An open question remains as to whether the required power expenditures for such devices can be achieved for practical systems. This chapter addresses this question by presenting results from a numerical study for hypersonic flow over a blunt-nose elliptic cone geometry with a thermal actuator. The thermal actuator deposits energy into the flow and is represented as a phenomenological dissipative heating model, as outlined below. The study investigates how the shape, location, and input power of deposition affect vehicle control. In addition, surface temperature and additional vehicle configurations are explored to draw conclusions over different flight regimes.

\subsection{Phenomenological Heating Model}

Flow-field results are obtained using the Navier-Stokes solver, LeMANS, outlined in Chapter II. A thermal actuator is considered as the plasma control device in this study. It is represented as a phenomenological model of dissipative heating and is 
accounted for in the total energy equation as an additional source term $S$, on the right hand side of Eqn. 3.1:

$$
\frac{\partial E}{\partial t}+\nabla \cdot\left((E+p) \mathbf{u}-\tau \cdot \mathbf{u}+\mathbf{q}+\Sigma\left(\mathbf{J}_{s} h_{s}\right)\right)=S
$$

where $E$ is the total energy per unit volume of the gas mixture, $p$ is the pressure, $\mathbf{u}$ is the bulk velocity vector, $\tau$ is the shear stress tensor, and $\mathbf{q}$ is total heat flux vector. The species diffusion flux, $\mathbf{J}_{s}$, represents the species inclination to move from regions of high concentration into regions of low concentration. The quantity $h_{s}$, is the species enthalpy, a thermodynamic property derived from the first law of thermodynamics [65], and is approximated using curve fits outlined in [67].

The vibrational-electron-electronic energy equation, Eqn. 2.6, is unaltered due to the addition of the phenomenological source term in the total energy equation. Therefore, during thermal nonequilibrium simulations, $100 \%$ of the heat deposition goes initally into translational energy. Depositing all of the dissipative heating energy into the translational energy mode is a strong assumption, but it is unclear how much of the energy should be directly deposited into the vibrational-electron-electronic mode as different types of thermal actuators have unique performance characteristics, and the present model is adequate for the purpose of illustrating the effects of thermal nonequilibrium.

During thermal nonequilibrium simulations, energy transfers into the vibrationalelectron-electronic energy mode by means of the source term $\dot{\omega}_{v e}$. The vibrationalelectron-electronic energy source term, $\dot{\omega}_{v e}$, is an approximation to the vibrationalelectron-electronic work done by the production and destruction of species due to chemistry, energy transfer between nonequilibrium modes, and work done by electrons due to the electric field induced by the electron pressure gradient. Full details 
of the vibrational-electron-electronic source term are available in [67].

The shape and location of the actuator are modeled such that contours of constant energy deposition have the shape of a spheroid, or ellipsoid of revolution, based on computational work by Poggie [79]. The strength or total power deposited into the flow decays exponentially from the centroid of the ellipsoid, as seen in Eqn. 3.2:

$$
S=\frac{Q}{\pi^{3 / 2} a b c} \exp \left(-\left(\frac{\hat{x}}{a}\right)^{2}-\left(\frac{\hat{y}}{b}\right)^{2}-\left(\frac{\hat{z}}{c}\right)^{2}\right)
$$

where

$$
\begin{aligned}
& \hat{x}=\left(x-x_{c}\right) \cos \phi-\left(z-z_{c}\right) \sin \phi \\
& \hat{y}=\left(y-y_{c}\right) \\
& \hat{z}=\left(x-x_{c}\right) \sin \phi+\left(z-z_{c}\right) \cos \phi
\end{aligned}
$$

Variables $a$ and $b$ are the equatorial radii (along the $x$ and $y$ axes), and $c$ is the polar radius (along the $z$-axis for an ellipsoid with $0^{\circ}$ inclination to the freestream). The variable $\phi$ is the angle between the major axis of the deposition region and the freestream flow. Coordinates $\left(x_{c}, y_{c}, z_{c}\right)$ represent the centroid of the deposition region. This formulation allows the shape of a contour of constant $S$ to change from sphere $(a=b=c)$ to an oblate spheroid $(a \approx b>c)$ to a prolate spheroid $(a \approx c<b)$ by specifying $a, b$, and $c$. Note that $Q$ represents the total power deposited in the flow: $\iiint_{-\infty}^{\infty} S d x d y d z=Q$.

\subsection{Validation}

Implementation of the phenomenological model is verified by simulating nonreacting nitrogen, $\mathrm{N}_{2}$, in a free domain with volumetric energy deposition. The purpose of the simulation is to verify that the amount of energy being deposited is equal to the amount leaving the domain for a converged steady-state solution. 
A nonuniform structured grid is generated using GAMBIT [80], with cell clustering in the region of deposition, in order to accurately capture the deposition shape. A cubic domain with a side length of $10 \mathrm{~cm}$ is represented using 20 fluid cells as seen in Fig. 3.1. A second, doubly refined grid is also employed to verify that the solution approaches grid independence.

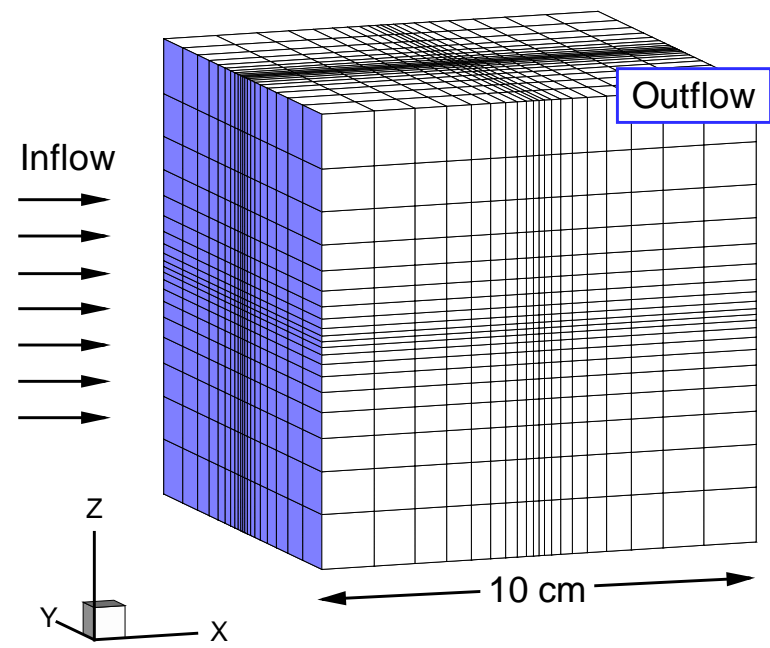

Figure 3.1: Geometry and grid for validation of volumetric energy deposition in a free domain.

A spherical shape is employed for the heating source, with its centroid located in the center of the domain $\left(x_{c}, y_{c}, z_{c}\right)=(0,0,0)$. The freestream conditions and parameters defining the sphere are listed in Table 3.1.

Table 3.1: Flow-field conditions for the volumetric heating validation case in a free domain.

\begin{tabular}{ccccc|ccc}
\hline \hline \multicolumn{4}{c}{ Freestream } & \multicolumn{3}{c}{ Deposition } \\
\hline Gas & Mach & $\mathbf{T}_{\infty}$ & $\rho_{\infty}$ & $u_{\infty}$ & $a$ & $b$ & $c$ \\
\hline $\mathrm{N}_{2}$ & 3.1 & $250 \mathrm{~K}$ & $0.1 \mathrm{~kg} / \mathrm{m}^{3}$ & $1000 \mathrm{~m} / \mathrm{s}$ & $0.005 \mathrm{~m}$ & $0.005 \mathrm{~m}$ & $0.005 \mathrm{~m}$ \\
\hline \hline
\end{tabular}

The phenomenological heating model deposits energy into the domain which pro- 
duces an increase in the local temperature. As the solution converges to steady state, the heated gas moves downstream and exits through the outlet plane as seen in Fig. 3.2.

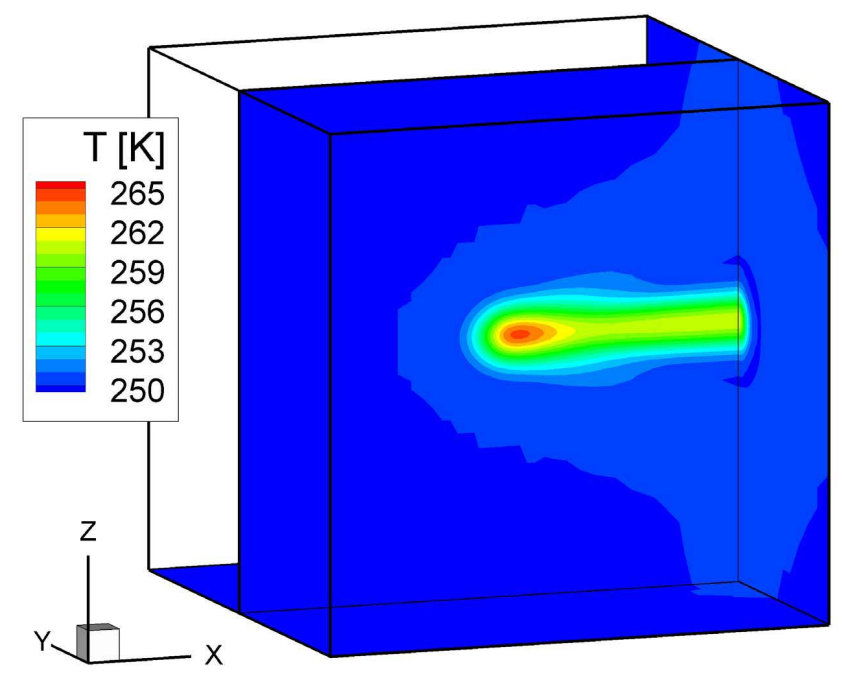

Figure 3.2: Temperature contours for Mach 3.1 nitrogen flow along the centerline of a free domain $(y=0)$, with a spherical energy deposition $Q=100 \mathrm{~W}$. $(40 \times 40 \times 40)$

To ensure the heat deposition is occurring correctly, the total energy increase is computed at the exit plane by relating the temperature gradient increase to the specific heat equation in Eqn. 3.4:

$$
Q=\int c_{v} \rho \Delta \mathrm{T}
$$

where $c_{v}$ is the specific heat at constant volume, $\rho$ is the gas density, and $\Delta \mathrm{T}$ is the change in temperature. Since the freestream temperature is $250 \mathrm{~K}$ and the amount of energy being deposited is small, the specific heat of molecular nitrogen is determined by using the Equipartition theorem and assuming a nitrogen molecule only has three translational and two rotational degrees of freedom, as seen in Eqn. 3.5: 


$$
c_{v}=\frac{3+2}{2} \frac{R}{m_{\mathrm{N}_{2}}}
$$

where the universal gas constant, $R=8314 \mathrm{~J} / \mathrm{kmol} \mathrm{K}$, and the mass per mole of nitrogen is $m_{\mathrm{N}_{2}}=28 \mathrm{~kg} / \mathrm{kmol}$. The total amount of additional heat energy leaving the domain is tabulated in Table 3.2 for each of the grids.

Table 3.2: Power loss through the free domain boundaries for $Q=100 \mathrm{~W}$.

\begin{tabular}{cc}
\hline \hline Grid & Q $_{\text {boundaries }}[\mathbf{W}]$ \\
\hline $20 \times 20 \times 20$ & 96.92 \\
$40 \times 40 \times 40$ & 99.22 \\
\hline \hline
\end{tabular}

The coarse grid $(20 \times 20 \times 20)$, does not have adequate resolution to account for all the energy, while the finer grid is much closer. These results verify that the additional energy is being correctly deposited into the flow.

\subsection{Energy Deposition Results}

The trim pitching moment is evaluated to determine the power expenditures necessary for a realistic hypersonic flight control system. The trim pitching moment is traditionally achieved by extending a control surface (flap), as seen on the wings of an F-22 Raptor in Fig. 3.3. The extended flap creates a torque (force $\times$ length measured from the center of gravity), and is necessary to maintain steady flight. For this investigation, the trim moment, traditionally supplied by the extended flap, is achieved by depositing energy into the flow, near the vehicle surface, using the phenomenological heating model previously described. 


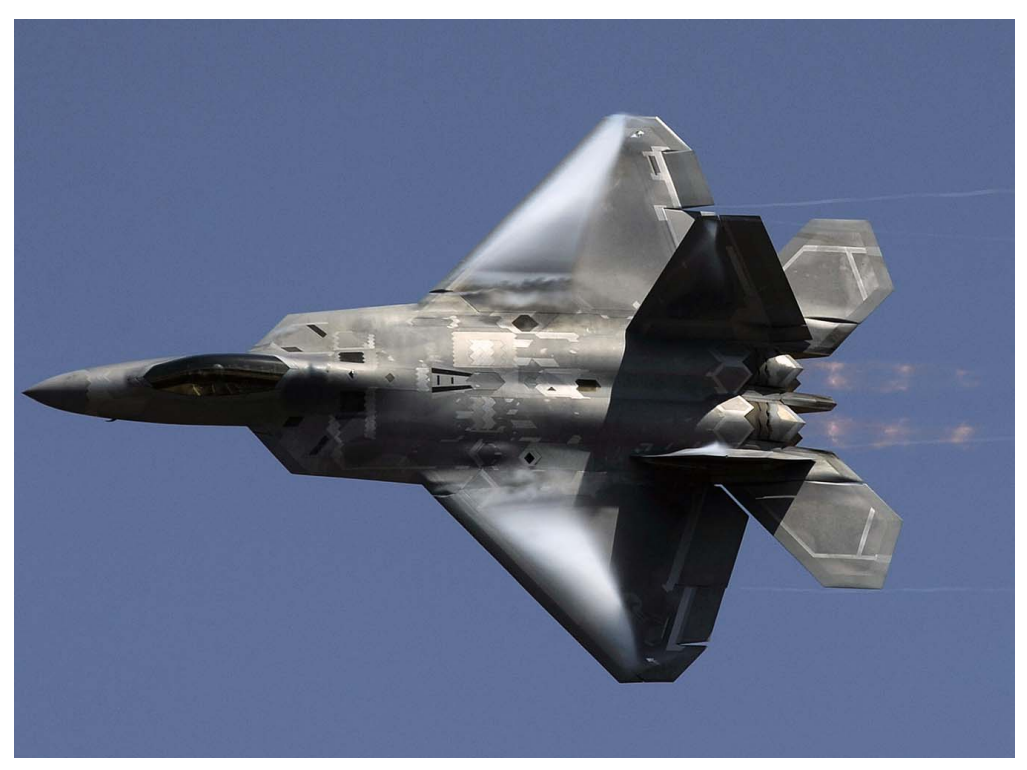

Figure 3.3: Steady flight (vehicle trim), is achieved by extending the flaps a small angle for the F-22 Raptor, a supersonic fighter aircraft [81].

\subsubsection{Reference Geometry}

The blunt-nose elliptic cone geometry considered in Section 2.2.2, scaled to a length of $L=3 \mathrm{~m}$, is selected as a representative hypersonic vehicle. Assuming the vehicle has uniform density, its center of gravity $(\mathrm{CG})$ is located $1.95 \mathrm{~m}$ from the stagnation point along the $x$-axis $\left(x_{\mathrm{CG}} / L=0.65\right)$. The model is simulated with freestream conditions consistent with air at $40 \mathrm{~km}$ altitude, a freestream velocity of $4000 \mathrm{~m} / \mathrm{s}$, and $0^{\circ}$ angle of attack. The complete flow conditions are provided in Table

\section{3.}

A structured grid, similar to the one described in Section 2.2.2, is employed for the simulations. Because planes of symmetry exist along the vehicle's major and minor axes, only one quarter of the geometry is represented in the domain. The model is aligned with the $x$-axis in the axial direction, the $y$-axis in the horizontal direction, and the $z$-axis in the vertical direction. To illustrate flow features along surface rays, a cylindrical coordinate system is also utilized with $\theta=0^{\circ}$ at the top 
Table 3.3: Flow conditions for Mach 12.6 air flow at an altitude of $40 \mathrm{~km}$.

\begin{tabular}{crl}
\hline \hline Parameter & \multicolumn{2}{c}{ Value } \\
\hline Mach & 12.6 & \\
$u_{\infty}$ & 4000.0 & $\mathrm{~m} / \mathrm{s}$ \\
$\mathrm{T}_{\infty}$ & $250.0 \mathrm{~K}$ \\
$\mathrm{~T}_{w}$ & $300.0 \mathrm{~K}$ \\
$\mathrm{~T}_{0}$ & $8300.0 \mathrm{~K}$ \\
$p_{\infty}$ & 289.0 & $\mathrm{~Pa}$ \\
$\rho_{\infty}$ & 0.004 & $\mathrm{~kg} / \mathrm{m}^{3}$ \\
$\mu_{\infty}$ & $1.6 \times 10^{-5}$ & $\mathrm{~kg} / \mathrm{m} \cdot \mathrm{s}$ \\
$\operatorname{Re} / x$ & $10^{6}$ & $\mathrm{~m}{ }^{-1}$ \\
\hline \hline
\end{tabular}

centerline of the model ( $z$-axis) and $\theta=90^{\circ}$ at the leading edge ( $y$-axis).

Although the new geometry has identically scaled features to the blunt-nose elliptic cone studied in Section 2.2.2, the new scale warrants a new grid independence study to ensure solutions are not influenced by the mesh. A nonuniform grid is developed with a gradual increase in mesh spacing along the axial direction with the smallest spacing in the stagnation region. Radial points are algebraically spaced to increase the number of points near the geometry surface. Grid points are equally spaced along the circumference. As a result, cell clustering occurs near the body surface and in the stagnation region to help capture the shape of the strong bow shock.

A grid independence study is conducted with $i \times j \times k$ dimensions changing from $150 \times 30 \times 30($ coarse $)$, to $300 \times 60 \times 60($ medium $)$, to $380 \times 80 \times 80$ (fine), to $400 \times$ $80 \times 120$ (very fine). The pressure coefficient, $\mathrm{Cp}$, and the Stanton number, St, are computed along the surface of the geometry to evaluate whether the flow properties most importance for this work, namely the surface heat transfer (flux) and pressure, 
are independent of the grid selected. The definitions of pressure coefficient and Stanton number are given in Eqns. 2.7 and 2.8, respectively. Figure 3.4 shows very little change in Cp or St between the 'fine' and 'very fine' grids. Grid independence is achieved with the 'fine' grid $(380 \times 80 \times 80)$, and is used in the remaining simulations presented in this chapter, unless otherwise stated.

\subsubsection{Reference Pitching Moment}

A nominal reference pitching moment is computed by assuming that a flap with dimensions of $0.2 \mathrm{~m} \times 0.5 \mathrm{~m}$ is attached to the aft of the vehicle along its top centerline. The flap is extended $2^{\circ}$ from its closed position as illustrated in Fig. 3.5. The size and location of the flap are based on illustrations of a hypersonic test vehicle shown in [82]. The control authority (trim pitching moment), provided by the extended mechanical flap is estimated computationally by incorporating the flap geometry with the blunt-nose elliptic cone geometry.

The flap geometry is combined with the reference blunt-nose elliptic cone geometry by flaring the last $0.2 \mathrm{~m}$ of the reference geometry. The flare extends around the circumference of the cone and has a $2^{\circ}$ inclination from its original location. Extending the flare around the circumference simplifies the geometry and eliminates unnecessary complexities (i.e. modeling the edge of the extended flap). Figure 3.6 shows the resultant pressure increases at the flare.

Since the spanwise width of the flap extends $0.25 \mathrm{~m}\left(\theta=18^{\circ}\right)$ from the top centerline $\left(\theta=0^{\circ}\right)$, the control authority provided by the flap is estimated by multiplying the local increase in body force on the flap surface by its moment arm $(1.05 \mathrm{~m})$. This results in a reference pitching moment, $M_{p_{\text {flap }}}=22.8 \mathrm{~N}-\mathrm{m}$. Although this flap configuration is hypothetical, its solution is adequate for interpreting results obtained 


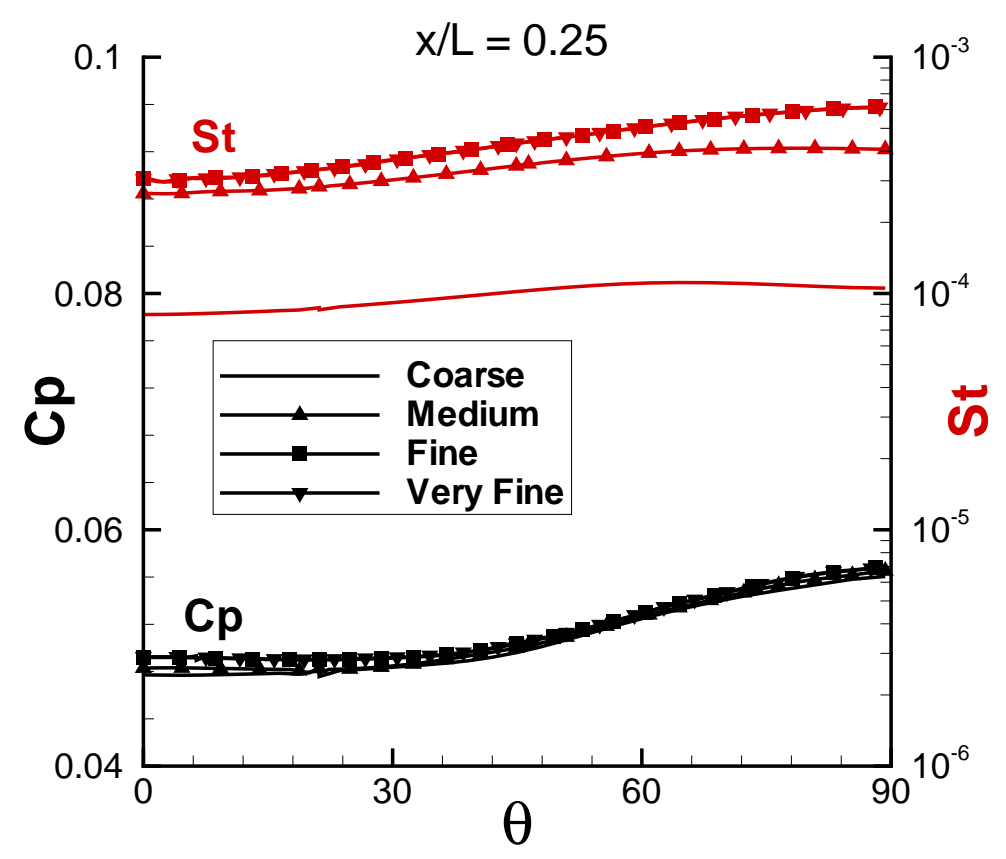

(a) Around the circumference.

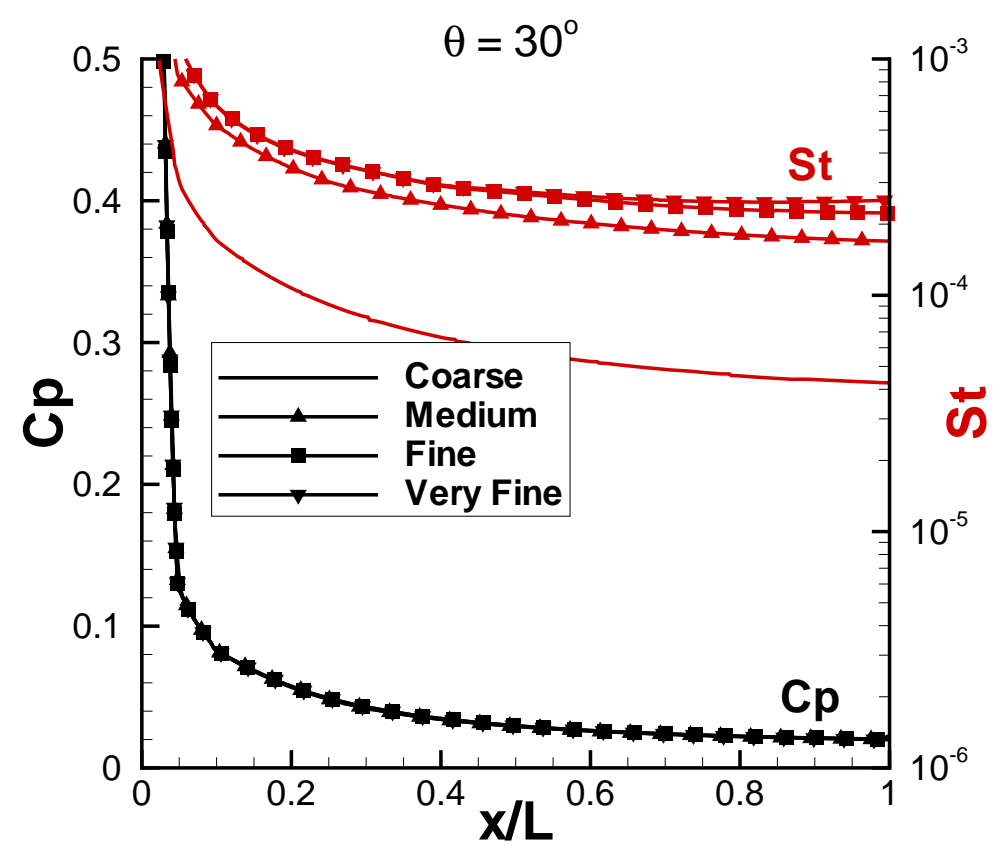

(b) Along rays.

Figure 3.4: Grid independence study for Mach 12.6 air flow around a blunt-nose elliptic cone $(L=3 \mathrm{~m})$. 


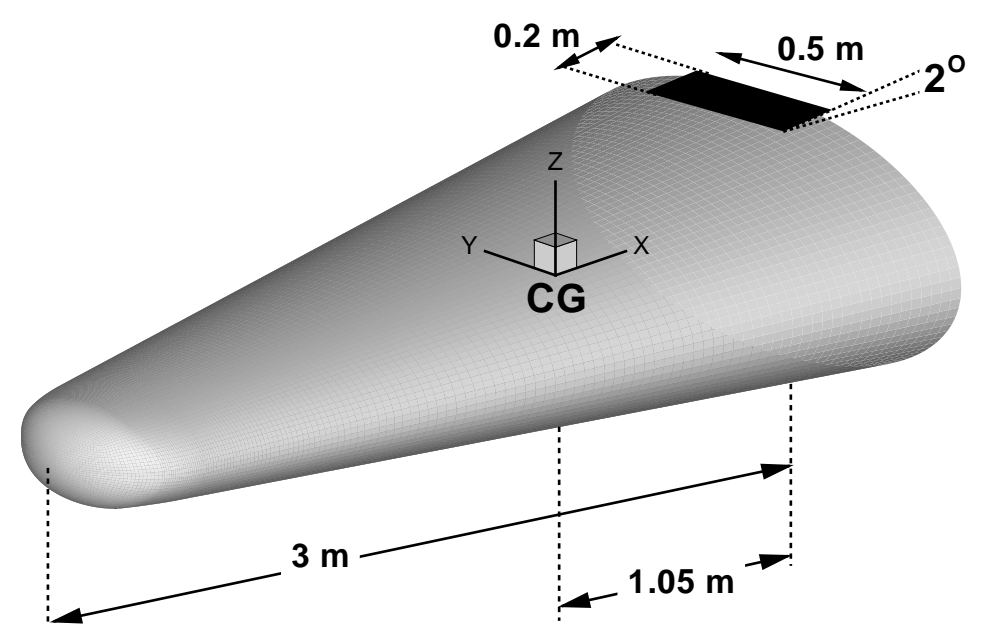

Figure 3.5: Blunt-nose elliptic cone geometry with an extended $2^{\circ}$ flap.

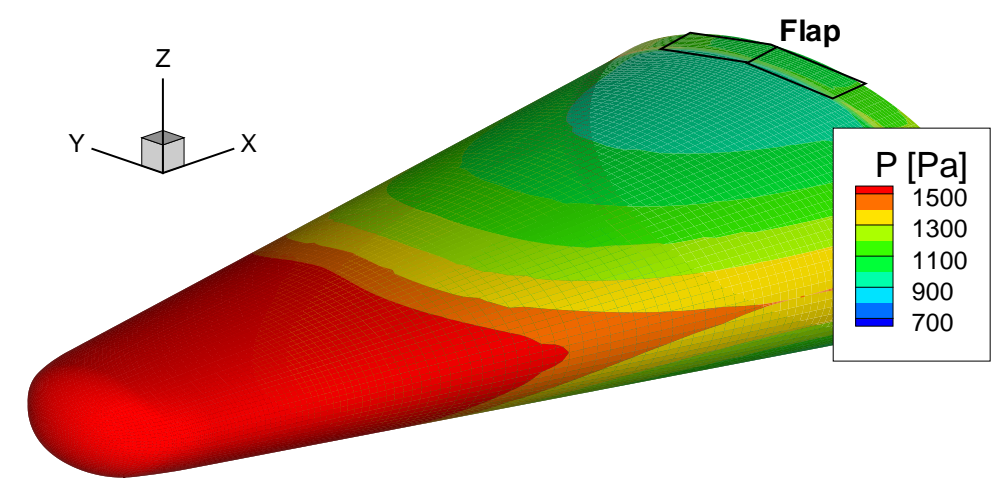

Figure 3.6: Pressure contours for Mach 12.6 air flow around a blunt-nose elliptic geometry with $2^{\circ}$ flare $2.8 \mathrm{~m}$ from the leading edge. 
by phenomenological energy deposition and can be replaced with another reference pitching moment for different vehicle design requirements.

\subsubsection{Study Parameters}

To limit the scope of the problem, three volumetric deposition shapes are selected. Namely a sphere, an oblate spheroid, and a prolate spheroid are employed such that a representative volume of the energy deposition region remains constant $(4 / 3 \pi a b c=$ constant). The oblate spheroid's equatorial radii are equal $(a=b)$ while its polar radius is smaller $(c<a)$. This selection of parameters flattens the spheroid to resemble a disk-like or pancake shape, with the equatorial plane parallel to the body surface. For the prolate spheroid, the polar radius and one of the equatorial radii are set equal $(a=c)$ while the other equatorial radius is larger $(b>a)$. This selection of parameters stretches the spheroid outward from the geometry's center-plane $(y=0)$ to resemble a football or bean, with the equatorial plane perpendicular to the body surface. The values controlling the shape of the spheroid deposition are listed in Table 3.4.

Table 3.4: Ellipsoid deposition parameters for Mach 12.6 air flow around a blunt-nose elliptic cone $(L=3 \mathrm{~m})$.

\begin{tabular}{cccc}
\hline \hline Shape & $a$ & $b$ & $c$ \\
\hline Sphere & $0.007 \mathrm{~m}$ & $0.007 \mathrm{~m}$ & $0.007 \mathrm{~m}$ \\
Oblate spheroid & $0.01852 \mathrm{~m}$ & $0.01852 \mathrm{~m}$ & $0.001 \mathrm{~m}$ \\
Prolate spheroid & $0.001852 \mathrm{~m}$ & $0.1 \mathrm{~m}$ & $0.001852 \mathrm{~m}$ \\
\hline \hline
\end{tabular}

The centroid of the deposition is positioned along the top centerline $\left(y_{c}=0 \mathrm{~m}\right)$ and is at least three characteristic length scales $\lambda$ from the geometry surface to ensure all the energy is deposited into the flow-field $\left(\iiint_{-3 \lambda}^{3 \lambda} S d x d y d z=0.9999 Q\right)$. This 
distance is the minimum length from the centroid of a spherical deposition to the surface as illustrated in Fig. 3.7.

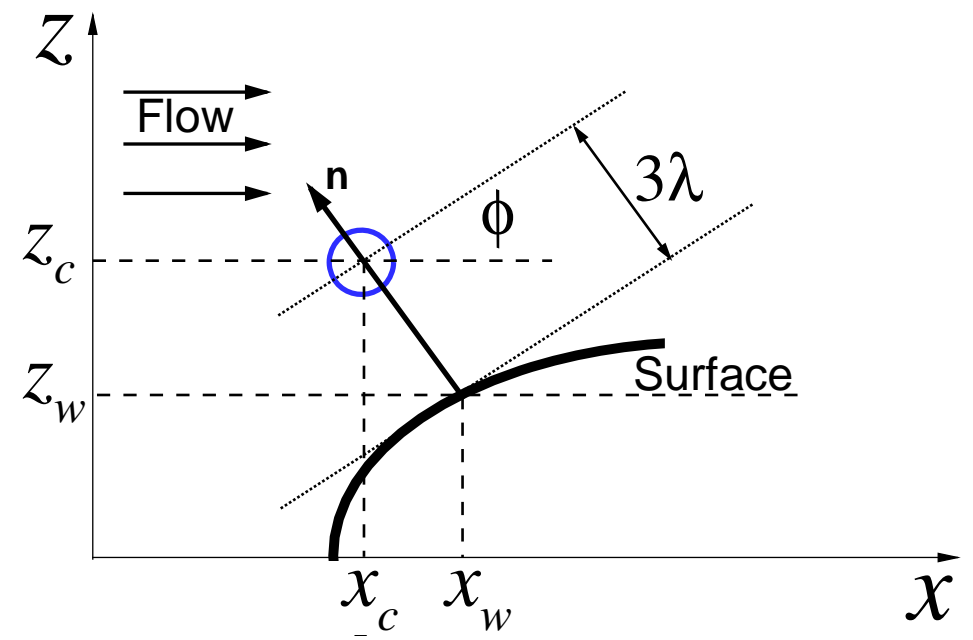

Figure 3.7: Three characteristic length scales $(3 \lambda)$, are used to determine $z_{c}$ and $\phi$ for the phenomenological energy deposition study.

The axial location of the deposition $x_{c}$ is set as an input condition which allows for the value of $z_{c}$ to be determined for $3 \lambda=3 a$ by enforcing Eqns. 3.6 and 3.7:

$$
\begin{aligned}
& x_{c}=x_{\mathrm{w}}+3 \lambda \cdot\left\|n_{x_{\mathrm{w}}}\right\| \\
& z_{c}=z_{\mathrm{w}}+3 \lambda \cdot\left\|n_{z_{\mathrm{w}}}\right\|
\end{aligned}
$$

where $\left(x_{\mathrm{w}}, z_{\mathrm{w}}\right)$ is the geometry surface location where the outward normal vector $\mathbf{n}$ extends through the centroid of the deposition $\left(x_{c}, y_{c}, z_{c}\right)$ and corresponds to Fig. 3.7. Equation 3.8 determines $\phi$ so that the polar radius of the ellipsoidal deposition aligns with n:

$$
\phi=\tan ^{-1}\left\|\frac{n_{x_{\mathrm{w}}}}{n_{z_{\mathrm{w}}}}\right\|
$$


The oblate spheroid is positioned so its major axis is parallel to the freestream flow, whereas the major axis of the prolate spheroid is perpendicular to the freestream flow. Figure 3.8 illustrates the shape of each deposition for a contour of constant $S$.

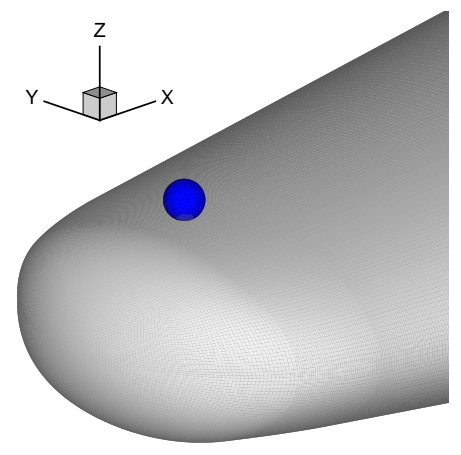

(a) Sphere deposition

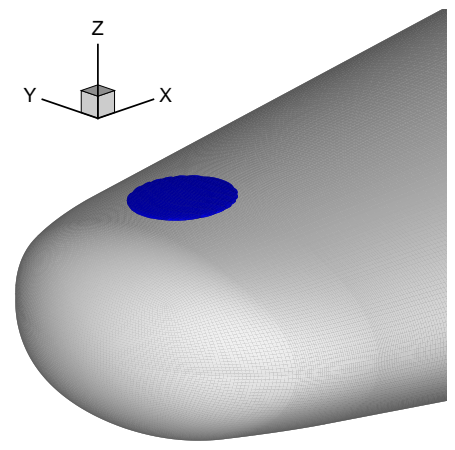

(b) Oblate deposition

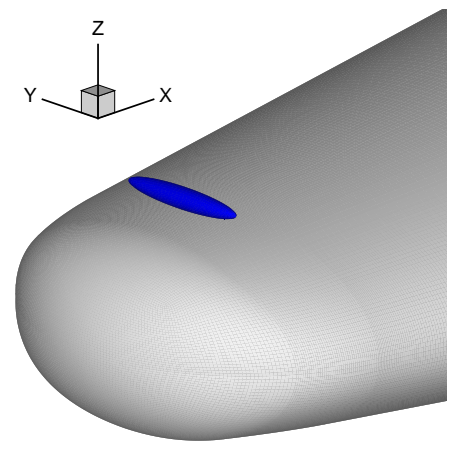

(c) Prolate deposition

Figure 3.8: Contours of constant $S$ for the various ellipsoid deposition shapes employed for Mach 12.6 air flow around a blunt-nose elliptic cone geometry $(L=3 \mathrm{~m})$.

Using the Mach 5 flat plate experiment studied by Kimmel et al. [28, 83] and a recent magnetohydrodynamic power generation experiment [84] for reference, realistic power input is assumed to lie in the range of $1 \mathrm{~kW}$ to $15 \mathrm{~kW}$. The deposition is positioned near the nose of the geometry to maximize the distance from the center of gravity (CG). This is done not only to increase the moment arm of the body force due to the deposition, but also because larger force changes are observed when a plasma-based actuator is placed near the bow shock [40]. The three deposition shapes are studied in combination with two additional parameters: the deposition input power $Q$ and the deposition centroid distance along the body $x_{\mathrm{w}} / L$.

In order to provide some information on vehicle and application scaling, the total power deposited into the flow is characterized by the nondimensional total power deposition value $\tilde{Q}$ as defined in Eqn. 3.9: 


$$
\tilde{Q}=\frac{Q}{\rho_{\infty} u_{\infty}^{3} L^{2}}
$$

where $Q$ is the total power deposited and $L$ is the cone's length. For the cases examined in the study $\tilde{Q}=4.3 \times 10^{-7}, 1.7 \times 10^{-6}$, and $6.5 \times 10^{-6}$ for $Q=1 \mathrm{~kW}, 4$ $\mathrm{kW}$, and $15 \mathrm{~kW}$, respectively.

\subsubsection{Parametric Study Results}

The axial location of the energy deposition is apparent in the plots of pressure coefficient and Stanton number along the top centerline $\theta=0^{\circ}$ for the three deposition shapes in Fig. 3.9.

A slight increase in the Stanton number is accompanied by a more significant rise in the pressure coefficient, particularly in the sphere and oblate spheroid depositions. This may be due to the fact that the sphere has the minimal surface area, and consequently, has the highest power deposited per projected surface area onto the body. The total force acting on the surface in the Cartesian coordinate system is found using Cauchy's theorem with the total stress tensor containing the hydrostatic pressure as show in Eqn. 3.10:

$$
\mathbf{F}=\int(\tau-p I) \cdot \mathbf{n} d A
$$

where $\mathbf{F}$ is the force vector, $\tau$ is the shear stress tensor, $I$ is the identity matrix, $p$ is the pressure, $\mathbf{n}$ is the outward unit normal vector, and $d A$ is the surface area. With the local force known, the pitching moment $M_{p}$ is determined in the conventional manner. Because the deposition only occurs on the top half of the vehicle, the pitching moment for each scenario is the deviation from the reference (baseline) scenario $M_{p}=M_{p}$ (deposition) $-M_{p}$ (baseline). The solution is normalized by the moment of 


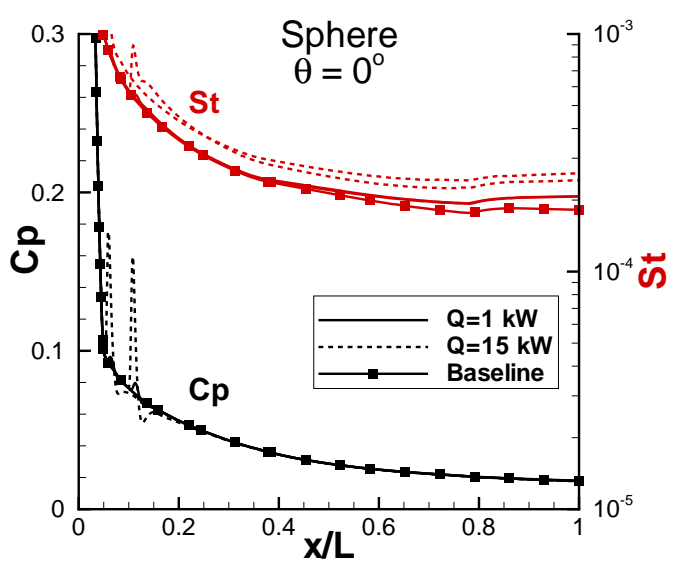

(a) Sphere deposition

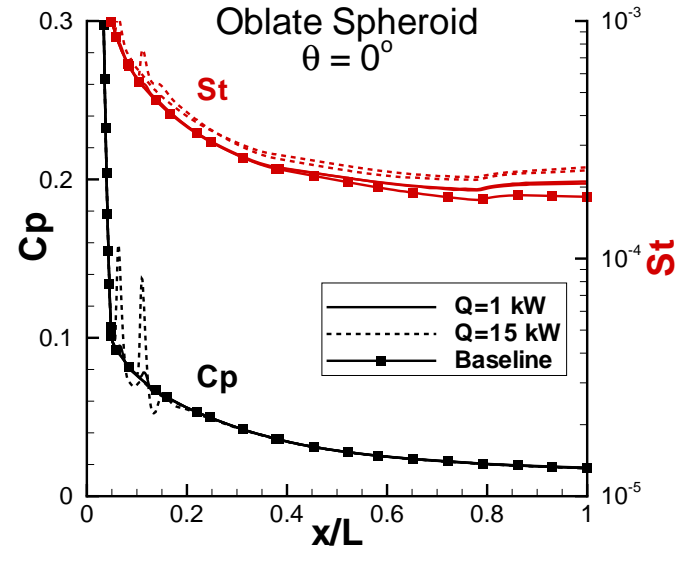

(b) Oblate spheroid deposition

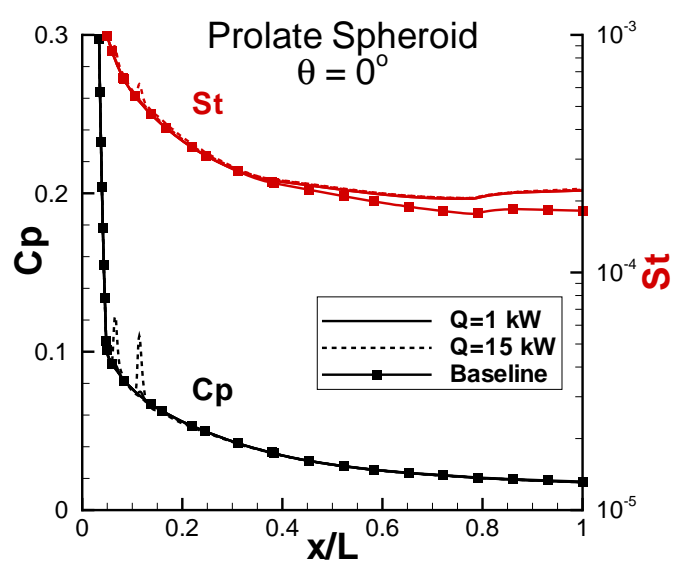

(c) Prolate spheroid deposition

Figure 3.9: Pressure coefficient and Stanton number distributions along the top centerline $\left(\theta=0^{\circ}\right)$, for Mach 12.6 air flow around a blunt-nose elliptic cone $(L=3 \mathrm{~m})$, for various energy deposition patterns $\left(\mathrm{T}_{\mathrm{w}}=300 \mathrm{~K}\right)$. 
the mechanical flap, $M_{p}$ (flap) $=22.8 \mathrm{~N}-\mathrm{m}$. Figure 3.10 plots the normalized pitching moment for each of the deposition shapes. The individual scenarios are fitted with a parametric spline because of their assumed non-linearity.

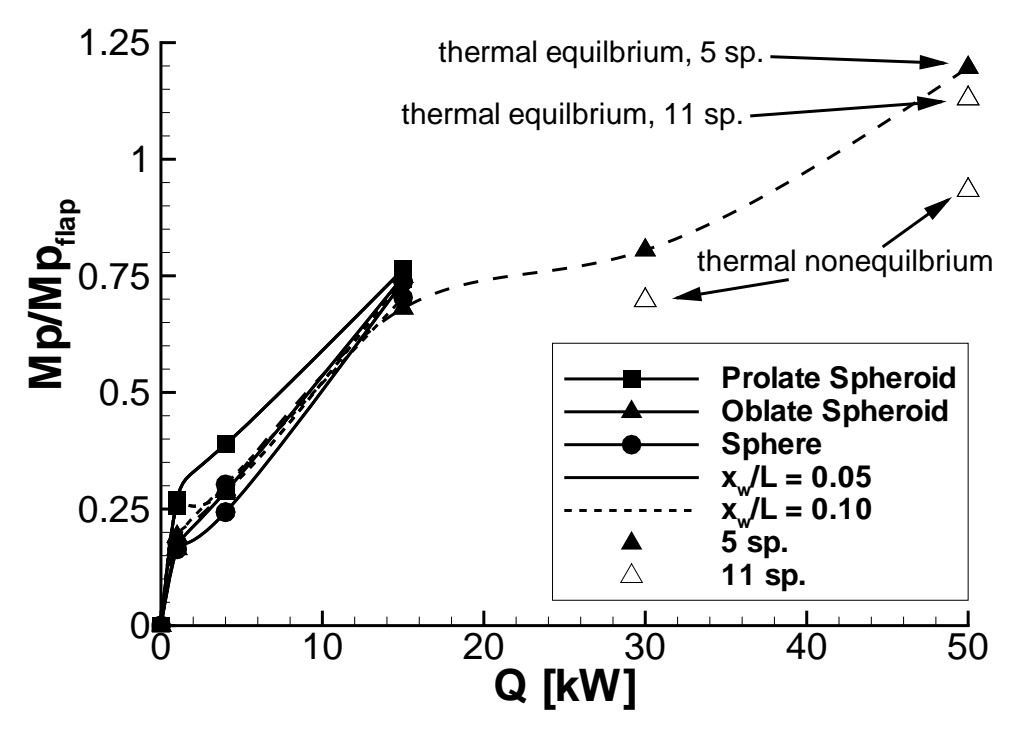

Figure 3.10: Normalized change in pitching moment for Mach 12.6 air flow around a blunt-nose elliptic cone $(L=3 \mathrm{~m})$, for various energy deposition patterns $\left(\mathrm{T}_{\mathrm{w}}=300 \mathrm{~K}\right)$.

All simulations are computed assuming thermal equilibrium and use a 5 species finite rate chemistry model $\left(\mathrm{N}_{2}, \mathrm{O}_{2}, \mathrm{NO}, \mathrm{N}\right.$, and $\left.\mathrm{O}\right)$, except in the two largest deposition scenarios $\left(Q=30,50 \mathrm{~kW}, x_{\mathrm{w}} / L=0.10\right.$, oblate spheroid). The $Q=50 \mathrm{~kW}$ scenario is repeated for two additional conditions: thermal equilibrium, 11 species chemistry model; and thermal nonequilibrium, 11 species chemistry model. The 11 species air chemistry model $\left(\mathrm{N}_{2}, \mathrm{O}_{2}, \mathrm{NO}, \mathrm{N}, \mathrm{O}, \mathrm{N}_{2}^{+}, \mathrm{O}_{2}^{+}, \mathrm{NO}^{+}, \mathrm{N}^{+}, \mathrm{O}^{+}, \mathrm{e}\right)$, accounts for weakly-ionized plasmas. As with the previous scenarios, these cases are compared against their respective baseline cases to determine the effectiveness of the deposition, $M_{p}=M_{p}$ (deposition) $-M_{p}$ (baseline).

For thermal equilibrium air, the inclusion of the expanded chemistry model has a relatively small impact on the total pitching moment as seen in the $Q=50 \mathrm{~kW}$ 
case (equilibrium, 5 sp. vs. equilibrium, 11 sp.). Thermal nonequilibrium noticeably reduces the effectiveness of the energy deposition because only the translational energy affects the local pressure. Comparing the results for the thermal equilibrium, 5 species simulations to the thermal nonequilibrium, 11 species simulations for the $Q$ $=30,50 \mathrm{~kW}$ scenarios, it is clear that thermal nonequilibrium and weakly-ionized plasma effects become increasing significant as the total power deposited increases and the flow deviates from a perfect gas.

Figure 3.10 shows energy deposition is able to provide the same order of magnitude of control authority as the mechanical flap. In addition, while the shape of the deposition appears to have noticeable effects on the local pressure coefficient and Stanton number, as seen in Fig. 3.9, it does not appear to significantly impact the overall change in the pitching moment provided to the vehicle.

\subsubsection{Hot Wall Effect}

The previous simulations assumed a constant wall temperature of $300 \mathrm{~K}$. This is significantly cooler than the expected wall temperature of a real hypersonic vehicle. Assuming the vehicle surface emissivity is equal to a blackbody $(\varepsilon=1)$, the StefanBoltzmann Law, Eqn. 3.11, is used along with the surface heat flux to estimate a wall temperature in radiative equilibrium:

$$
\mathrm{T}_{\mathrm{w}}=\left(\frac{\mathrm{q}_{\mathrm{w}}}{\varepsilon \sigma^{*}}\right)^{1 / 4}
$$

where $T_{w}$ is the wall temperature, $\mathrm{q}_{\mathrm{w}}$ is the total heat flux to the wall, and the Stefan-Boltzmann constant $\sigma^{*}=5.6704 \times 10^{-8} \mathrm{~W} / \mathrm{m}^{-2} \mathrm{~K}^{-4}$. Figure 3.11 shows that the surface temperature varies from $800 \mathrm{~K}$ to $2100 \mathrm{~K}$, with an average temperature of $1000 \mathrm{~K}$ in the region of the deposition $x_{\mathrm{w}} / L=0.10$. 


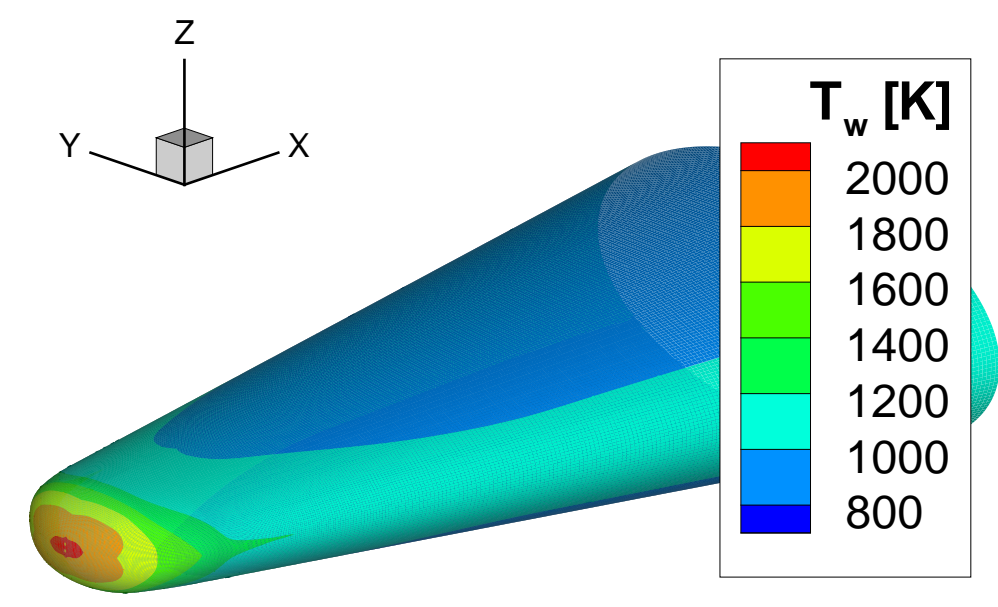

Figure 3.11: Radiative equilibrium surface temperature contours for Mach 12.6 air flow around a blunt-nose elliptic cone $(L=3 \mathrm{~m})$.

A new set of simulations is conducted with $\mathrm{T}_{\mathrm{w}}=1000 \mathrm{~K}$ and all other conditions equal to those listed in Table 3.3. The simulations use the oblate spheroid deposition parameters listed in Table 3.4 and a deposition centroid $x_{\mathrm{w}} / L=0.10$. Two additional simulations are also computed using the same parameters as the $\mathrm{T}_{\mathrm{w}}=1000 \mathrm{~K}$ scenarios, except the wall temperature is allowed to locally equilibrate to the radiative wall temperature previously described by Eqn. 3.11.

Figure 3.12 plots the normalized change in pitching moment versus power deposited for two constant wall temperatures and the radiative wall. The higher wall temperature reduces the pitching moment of the vehicle because a larger portion of the energy deposited goes into the higher energy modes (i.e. rotation, vibration, and dissociation) instead of the translational energy mode. The radiative wall case results lie between the two isothermal wall cases because the radiative wall lowers the temperature in the stagnation region and near the vehicle surface. 


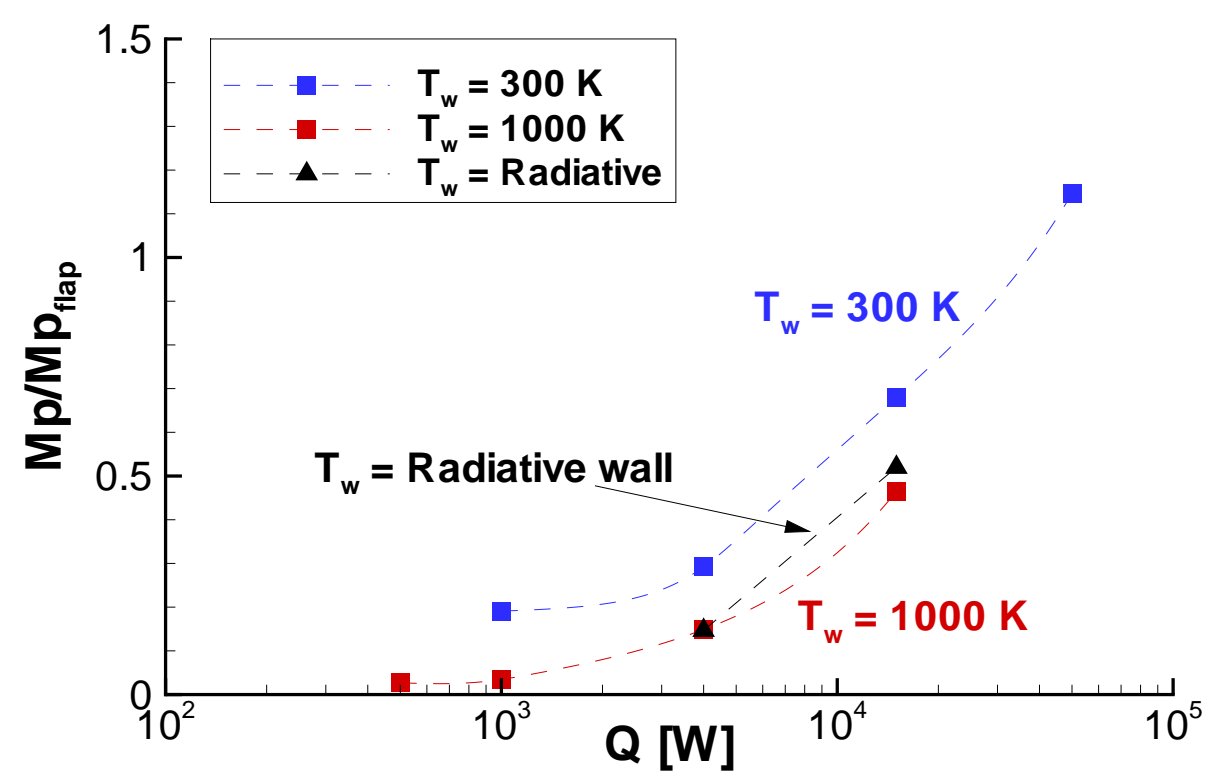

Figure 3.12: Normalized change in pitching moment for Mach 12.6 air flow around a blunt-nose elliptic cone $(L=3 \mathrm{~m})$, with an oblate spheroid energy deposition for two isothermal walls and a radiative equilibrium wall.

\subsection{Additional Vehicle Configurations}

The freestream conditions used in the simulations of the $L=3 \mathrm{~m}$ configuration (Table 3.3) are applied to another scaled geometry. The 'medium' scaled vehicle has a length $L=0.62 \mathrm{~m}$, three times larger than the blunt-nose elliptic geometry studied in Section 2.2.2. The grid used for the 'large' simulations $(380 \times 80 \times 80)$, is also applied for this configuration by scaling down the grid spacing to accommodate the smaller vehicle length. The solutions are assumed to be grid-independent because the freestream conditions are identical to the 'large' blunt-nose elliptic cone simulations, while the grid spacing decreases, which is equivalent to using a finer mesh.

The nondimensional total power deposition value $\tilde{Q}$ for the cases run is, $\tilde{Q}=$ $4.0 \times 10^{-5}, 1.0 \times 10^{-4}$, and $1.5 \times 10^{-4}$ for $Q=4 \mathrm{~kW}, 10 \mathrm{~kW}$, and $15 \mathrm{~kW}$, respectively. The deposition is modeled as an oblate spheroid, scaled to match the oblate spheroid 
in the $L=3 \mathrm{~m}$ parametric study. Table 3.5 lists the values used to represent the energy deposition volume with the deposition positioned near the bow shock $x_{\mathrm{w}} / L=0.10$.

Table 3.5: Ellipsoid deposition parameters for Mach 12.6 air flow around blunt-nose elliptic cone $(L=0.62 \mathrm{~m})$.

\begin{tabular}{ccc}
\hline \hline$a$ & $b$ & $c$ \\
\hline $0.00386 \mathrm{~m}$ & $0.00386 \mathrm{~m}$ & $0.00021 \mathrm{~m}$ \\
\hline \hline
\end{tabular}

The pitching moment due to the mechanical flap is determined following the approach covered in Section 3.4.2 with the flap dimensions proportionately contracted $(0.105 \mathrm{~m} \times 0.042 \mathrm{~m})$. The mechanical flap achieves a pitching moment, $M_{p_{\text {flap }}}=$ $0.148 \mathrm{~N}-\mathrm{m}$.

The smaller geometry produces a weaker bow shock and consequently a lower post shock temperature. Similar to the results presented in Section 3.4.5, the cooler temperature improves the control authority provided by energy deposition. However, a large spike in the Stanton number distribution is observed in Fig. 3.13. This coincides with the location of the deposition and partially recovers to the baseline distribution as the flow progresses along the body. The distribution cannot fully recover because of the additional energy added to the flow and the shorter vehicle length.

The effects of energy deposition are also simulated for a 'small' geometry with a reference length $L=0.21 \mathrm{~m}$. The freestream conditions and geometry are identical to the blunt-nose elliptic cone investigated in Section 2.2.2 so the grid-independent mesh $(300 \times 60 \times 60)$ developed in Section 2.2.2 is utilized for the following scenarios.

Although the 'small' geometry freestream conditions listed in Table 2.2 are from a 


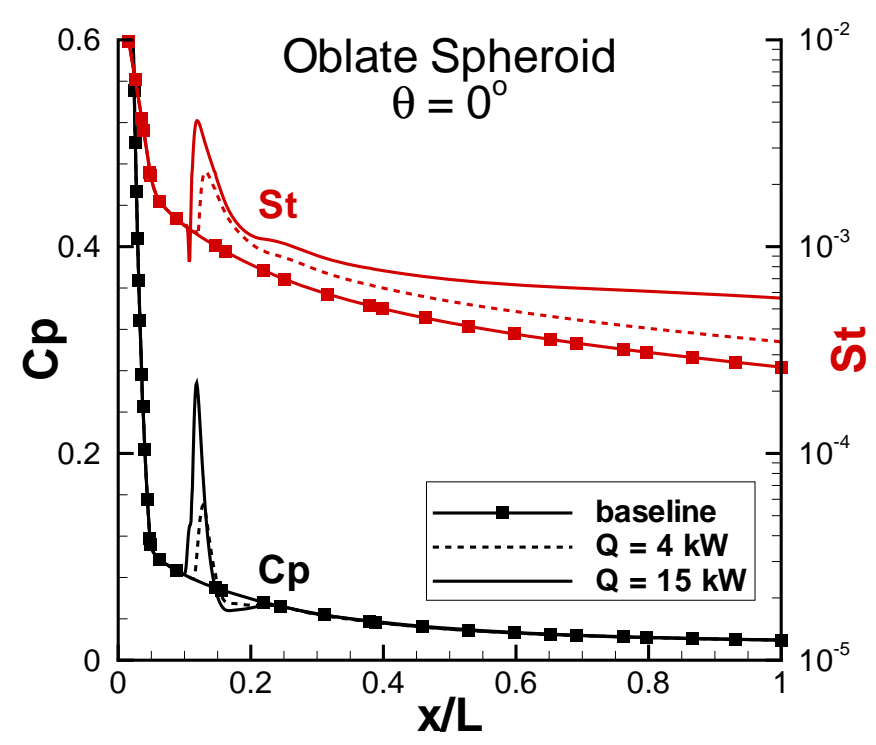

Figure 3.13: Pressure coefficient and Stanton number distributions along the top center line $\left(\theta=0^{\circ}\right)$, for Mach 12.6 air flow around a blunt-nose elliptic cone $(L=0.62 \mathrm{~m})$, with different amounts of energy deposition $\left(\mathrm{T}_{\mathrm{w}}=\right.$ $300 \mathrm{~K})$.

wind tunnel experiment where the freestream temperature is very cold and freestream pressure is extremely low $\left(\mathrm{T}_{\infty}=59.3 \mathrm{~K}, p_{\infty}=51.0 \mathrm{~Pa}\right)$, the conditions are compared to air at altitude by examining the unit Reynolds number as seen in Eqn. 3.12:

$$
R e_{\mathrm{unit}}=\frac{\rho_{\infty} u_{\infty}}{\mu_{\infty}} \times 1 \mathrm{~m}
$$

where $\rho_{\infty}, u_{\infty}$, and $\mu_{\infty}$ are the freestream density, velocity, and dynamic viscosity, respectively. Using Table 2.2, the 'small' cone scenario unit Reynolds number $R e_{\text {unit }}=1.53 \times 10^{6}$, which is comparable to the 'medium' and 'large' scenarios where $R e_{\text {unit }}=10^{6}$. (Recall that the 'medium' and 'large' scenarios are simulated with freestream conditions consistent with air at $40 \mathrm{~km}$ and a velocity of $4000 \mathrm{~m} / \mathrm{s}$.) The higher unit Reynolds number implies that the freestream conditions are similar to air at an altitude lower than $40 \mathrm{~km}$ since density decreases with altitude.

Using the 'small' geometry freestream velocity $u_{\infty}=2190 \mathrm{~m} / \mathrm{s}$, and kinematic vis- 
cosity $\nu_{\infty}=\mu_{\infty} / \rho_{\infty}=1.44 \times 10^{-} 3 \mathrm{~m}^{2} / \mathrm{s}$, the representative altitude in air is determined by comparing its kinematic viscosity to the Standard Atmosphere in Table 3.6. The 'small' geometry freestream conditions represent an altitude of $33.6 \mathrm{~km}$ in air.

Table 3.6: Properties of the U.S. Standard Atmosphere [85].

\begin{tabular}{cccc}
\hline \hline Altitude $[\mathrm{km}]$ & $\rho\left[\mathrm{kg} / \mathrm{m}^{3}\right]$ & $\mu[\mathrm{kg} / \mathrm{m} \mathrm{s}]$ & $\nu\left[\mathrm{m}^{2} / \mathrm{s}\right]$ \\
\hline 30 & $1.8410 \times 10^{-} 2$ & $1.4753 \times 10^{-} 5$ & $8.014 \times 10^{-4}$ \\
33 & $1.1573 \times 10^{-} 2$ & $1.4992 \times 10^{-} 5$ & $1.295 \times 10^{-3}$ \\
34 & $9.8874 \times 10^{-} 3$ & $1.5140 \times 10^{-} 5$ & $1.531 \times 10^{-3}$ \\
35 & $8.4634 \times 10^{-} 3$ & $1.5287 \times 10^{-} 5$ & $1.806 \times 10^{-3}$ \\
\hline \hline
\end{tabular}

The decrease in freestream velocity along with the significantly smaller geometry increases the nondimensional total power deposition value $\tilde{Q}$ by several orders of magnitude. For the cases run, $\tilde{Q}=4.0 \times 10^{-4}, 8.0 \times 10^{-4}$, and $1.6 \times 10^{-3}$ for $Q=$ $500 \mathrm{~W}, 1 \mathrm{~kW}$, and $2 \mathrm{~kW}$, respectively.

An oblate spheroid, similar to the one used in the 'large' blunt-nose elliptic cone study, is employed to represent the thermal actuator. Table 3.7 lists the values used to represent the spheroid and its centroid. Unlike the previous scenarios, the deposition contours at constant $S$ are larger (i.e. ${ }^{4 / 3} \pi a b c=\mathrm{V}_{\text {small }}>\mathrm{V}_{\text {large }}$ ), and its polar radius is not perfectly aligned to the surface normal vector $\mathbf{n}$. In addition, the energy deposition centroid is also positioned slightly farther from the bow shock $x_{\mathrm{w}} / L=0.14$. The results discovered in the parametric study presented in Section 3.4.4 suggest the deposition shape and location have a minimal effect on the resultant pitching moment and indicate the results from these simulations are relevant and particularly useful when drawing conclusions across different configurations.

Compared to the previous configurations, the 'small' geometry produces an even 
Table 3.7: Ellipsoid deposition parameters for Mach 14.2 air flow around blunt-nose elliptic cone $(L=0.21 \mathrm{~m})$.

\begin{tabular}{ccccccc}
\hline \hline$x_{c}$ & $y_{c}$ & $z_{c}$ & $a$ & $b$ & $c$ & $\theta$ \\
\hline $0.0292 \mathrm{~m}$ & $0 \mathrm{~m}$ & $0.017 \mathrm{~m}$ & $0.003 \mathrm{~m}$ & $0.004 \mathrm{~m}$ & $0.001 \mathrm{~m}$ & $0^{\circ}$ \\
\hline \hline
\end{tabular}

weaker bow shock, which further reduces the post shock temperature. In addition, the freestream temperature and total enthalpy are much lower (refer to Table 2.2). This allows for a greater portion of the deposition energy to remain in the translational energy mode. In addition, the short body vehicle results in the large temperature rise observed within the region of the deposition extending farther along the vehicle. The extended heating is apparent in the significant downstream temperatures observed in Figure 3.14, where the deposition is illustrated as the blue oblate spheroid centered above the top centerline near the leading bow shock.

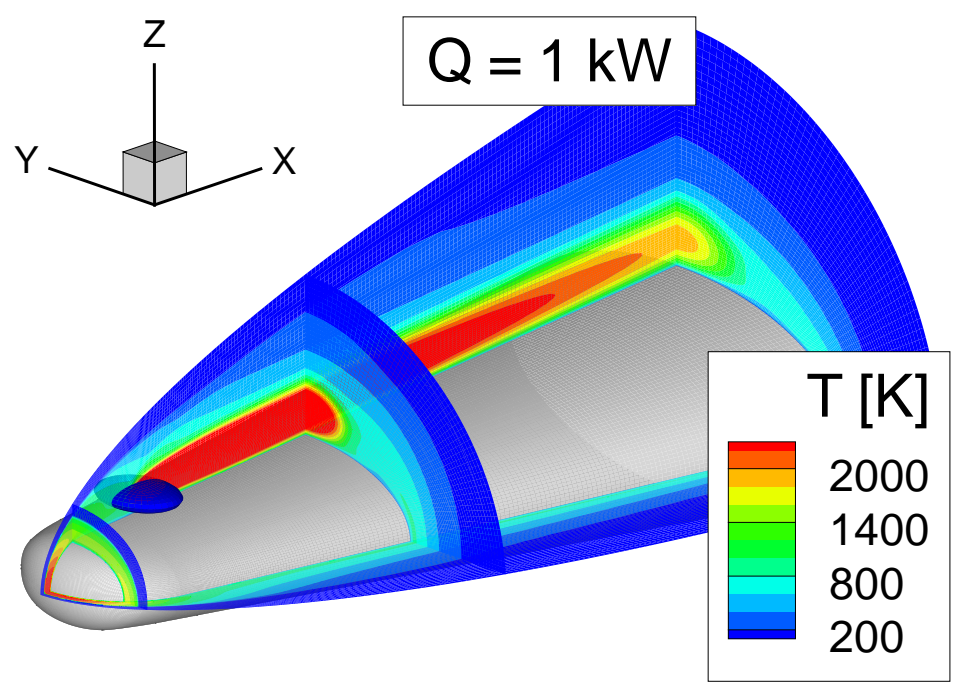

Figure 3.14: Temperature contours for Mach 14.2 air flow around a blunt-nose elliptic cone $(L=0.21 \mathrm{~m})$, with an oblate spheroid energy deposition $Q=1$ $\mathrm{kW}$.

Coinciding with the high temperature contours, a strong heat transfer penalty 
and dramatic increase in the pressure coefficient distribution are detected on the top centerline in Fig. 3.15. Although the Stanton number remains significantly elevated downstream of the deposition, the pressure coefficient quickly returns to the baseline (equilibrium) state which is consistent with the observations seen in the 'large' and 'medium' blunt-nose elliptic cone simulations.

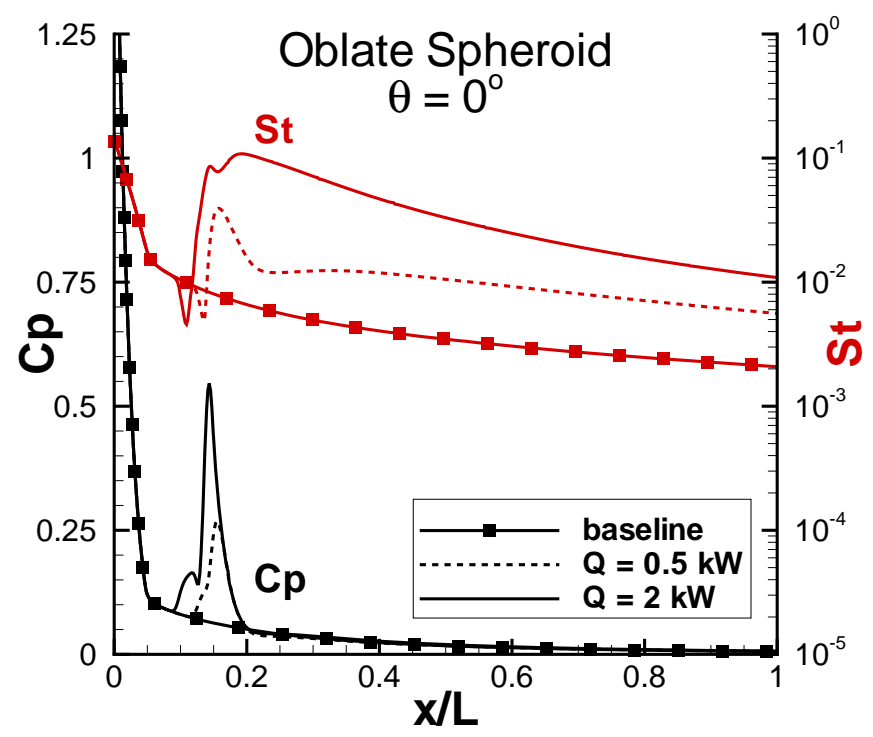

Figure 3.15: Pressure coefficient and Stanton number distributions along the top center line $\left(\theta=0^{\circ}\right)$, of Mach 14.2 air flow around a blunt-nose elliptic cone $(L=0.21 \mathrm{~m})$, with various levels of energy deposition $\left(\mathrm{T}_{\mathrm{w}}=294\right.$ $\mathrm{K})$.

Consistent with the procedure used previously, the pitching moment due to the mechanical flap is computed following the method presented in Section 3.4.2 with flap dimensions of $0.035 \mathrm{~m} \times 0.014 \mathrm{~m}$. This results in a reference pitching moment, $M_{p_{\text {flap }}}=9.16 \times 10^{-4} \mathrm{~N}-\mathrm{m}$.

To consolidate the results for all three geometries, the force produced by the energy deposition is also nondimensionalized using the moment coefficient defined in Eqn. 3.13: 


$$
C_{m}=\frac{M_{p}}{1 / 2 \rho_{\infty} u_{\infty}^{2} L^{2} d}
$$

where the pitching moment $M_{p}=M_{p}$ (deposition) $-M_{p}$ (baseline), $\rho_{\infty}$ and $u_{\infty}$ are the freestream density and velocity, respectively. The reference area is taken to be the vehicle maximum spanwise width $d$ multiplied by its length $L$. Table 3.8 lists the reference area for each of the geometries explored.

Table 3.8: Reference area for various the blunt-nose elliptic cone geometries.

\begin{tabular}{cccc}
\hline \hline Name & $L[\mathbf{m}]$ & $d[\mathbf{m}]$ & $L d\left[\mathbf{m}^{2}\right]$ \\
\hline Large & 3 & 1.644 & 4.932 \\
Medium & 0.62 & 0.343 & 0.213 \\
Small & 0.21 & 0.114 & 0.024 \\
\hline \hline
\end{tabular}

Figure 3.16 shows a strong correlation between the nondimensional total power deposition parameter $\tilde{Q}$ and the moment coefficient for the various simulations and geometries. Although the deposition shape used for the 'small' blunt-nose elliptic cone does not exactly match the 'medium' or 'large' simulations, the results further demonstrate the minimal contribution deposition shape and location have on the net control authority. The results appear to follow a nearly linear curve when plotted on a log-log scale $\left(C_{m} \approx \tilde{Q}^{1.1}\right)$. The different deposition locations, along with different vehicle lengths and freestream conditions, and real gas effects cause the results to deviate slightly from a power-law fit.

For all three geometries, the moment coefficient for the mechanical flap is computed to be $10^{-4}$ by using Eqn. 3.13. The log-log plot shows that energy deposition is a viable replacement for a mechanical flap when $\tilde{Q} \geq 10^{-4}$. Since $\tilde{Q}=Q / \rho_{\infty} u_{\infty}^{3} L^{2}$, smaller geometries, flying at lower velocities, and/or higher altitudes (lower densities) 


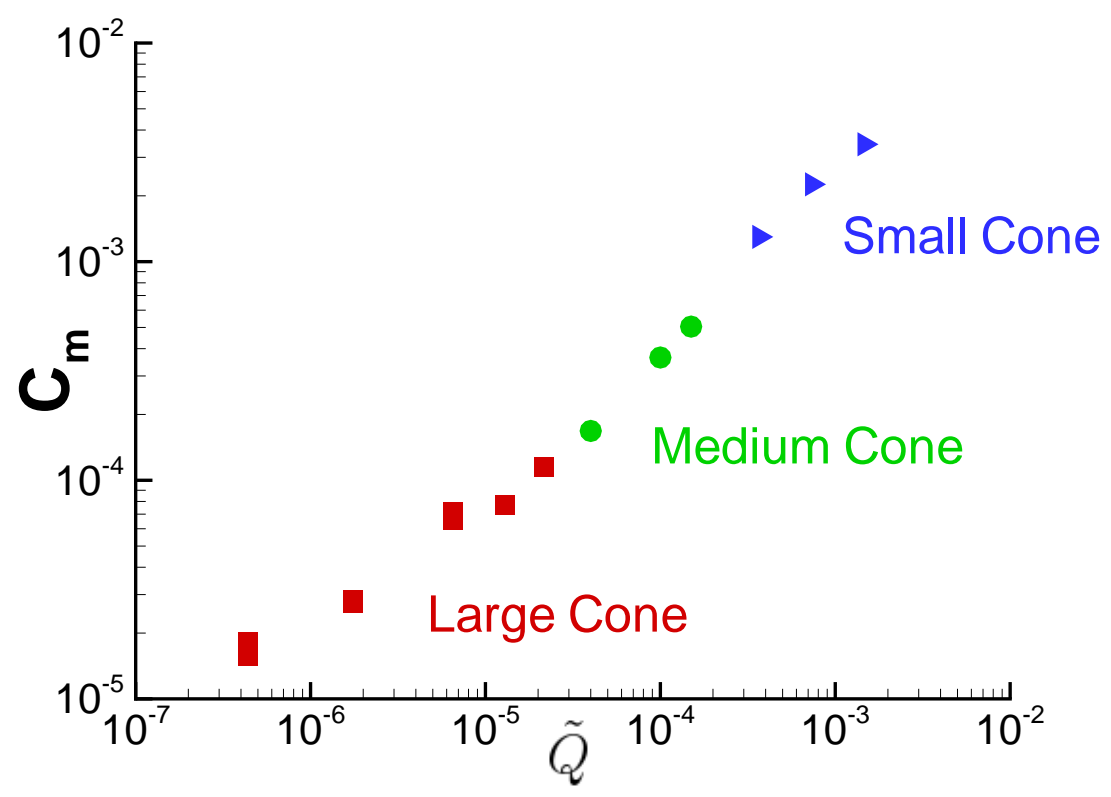

Figure 3.16: Moment coefficient versus the nondimensional total power deposition $(\tilde{Q})$, for various vehicle configurations $\left(\mathrm{T}_{\mathrm{w}} \simeq 300 \mathrm{~K}\right.$, thermal equilibrium, 5 sp., oblate spheroid deposition).

would make energy deposition a possible replacement for a mechanical flap. A table of the resultant pitching moment $M_{p}$ for all simulations is available in Appendix C.

\subsection{Conclusions}

In this chapter, a numerical study was performed to investigate whether a practical level of pitch moment control could be achieved from volumetric energy deposition for a realistic hypersonic vehicle. Using a phenomenological heating model, a parametric study was completed investigating the shape, location, and total amount of power volumetrically deposited into the flow-field for three blunt-nosed elliptic cone configurations. The shape of the deposition resulted in relatively small changes in the effectiveness of the deposition, whereas an increased wall temperature noticeably decreased the moment coefficient. Thermal nonequilibrium and weakly-ionized plasma effects also decreased the control authority as input power increases. The effective- 
ness of volumetric energy deposition for flight control appeared to scale strongly with a nondimensional parameter based on the freestream flow kinetic energy flux. Volumetric energy deposition appears to be a viable means of pitch control for configurations at higher altitude, with slower velocities, and smaller vehicle length. Note, however, that the penalty in additional heat transfer to the body increases as the control authority increases. 


\section{CHAPTER IV}

\section{Magnetohydrodynamics}

\subsection{Introduction}

A potential limitation of plasma-assisted devices is the large energy requirement necessary when they are employed to control large scale hypersonic flows by means of energy deposition [86]. One possible way of improving the effectiveness and/or providing finer control is to apply a force to the ionized portion of the flow. The ionized flow can be subjected to electric and magnetic fields, thereby producing additional and perhaps improved flow control. In order to simulate these effects, computational fluid codes need to be modified to accurately account for the magnetohydrodynamic (MHD) effects. This chapter outlines how the electromagnetic effects are accounted for within the flow solver by coupling it with a three-dimensional MHD solver. Several validation exercises are presented to show that the MHD module and boundary conditions are functioning correctly.

\subsection{Magnetohydrodynamic Model}

In Chapter III, a phenomenological model of dissipative heating is developed and implemented into the flow solver to model the effects of a thermal actuator for plasma-based control of a hypersonic vehicle [86]. While this approach can approx- 
imate the effects of a thermal actuator, the computational results near the device are not realistic. To remove this limitation and expand the types of devices that can be accurately simulated, including plasma-assisted devices that use electromagnetic fields, the phenomenological source term added to the right hand side of the modified total energy equation from Section 3.2, Eqn. 3.1, is replaced with an electromagnetic energy deposition term, $\mathbf{j} \cdot \mathbf{E}$, in Eqn. 4.1:

$$
\frac{\partial E}{\partial t}+\nabla \cdot\left((E+p) \mathbf{u}-\tau \cdot \mathbf{u}+\mathbf{q}+\Sigma\left(\mathbf{J}_{s} h_{s}\right)\right)=\mathbf{j} \cdot \mathbf{E}
$$

where $E$ is the total energy per unit volume of the gas mixture, $p$ is the pressure, $\mathbf{u}$ is the bulk velocity vector, $\tau$ is the shear stress tensor, and $\mathbf{q}$ is total heat flux vector. The species diffusion flux, $\mathbf{J}_{s}$, and enthalpy, $h_{s}$, are products of the finite chemistry models where force diffusion (drift) of the charged particles is neglected. The two additional variables are the current density, $\mathbf{j}$, and electric field, $\mathbf{E}$, vector.

The electromagnetic energy deposition term, $\mathbf{j} \cdot \mathbf{E}$, can be considered to consist of two components: a reversible work term, $(\mathbf{j} \times \mathbf{E}) \cdot \mathbf{u}$, and a dissipative term, $(\mathbf{E}+\mathbf{u} \times \mathbf{B}) \cdot \mathbf{j}$, the Joule heating. Physically, Joule heating is the kinetic and vibrational energy transfered by electrons when they collide with other particles (usually ions) [87]. The electrons are accelerated by the electric field. Joule heating is also known as resistive heating. Unlike in the phenomenological heating model, the vibrational-electron-electronic energy equation is also modified with the inclusion of a Joule heating term, $\gamma(\mathbf{E}+\mathbf{u} \times \mathbf{B}) \cdot \mathbf{j}$ in Eqn. 4.2:

$$
\frac{\partial E_{v e}}{\partial t}+\nabla \cdot\left(\left(E_{v e}\right) \mathbf{u}+\mathbf{q}_{v e}+\Sigma\left(\mathbf{J}_{s} e_{v e, s}\right)\right)=\dot{\omega}_{v e}+\gamma(\mathbf{E}+\mathbf{u} \times \mathbf{B}) \cdot \mathbf{j}
$$

where $E_{v e}$ is the vibrational-electron-electronic energy per unit volume of the gas mixture, $\mathbf{q}_{v e}$ is vibrational-electron-electronic heat flux vector, and $e_{v e, s}$ is the species 
vibrational-electron-electronic energy per unit mass. The vibrational energy source term, $\dot{\omega}_{v e}$, is an approximation to the vibrational-electronic work done by the production and destruction of species due to chemistry, energy transfer between nonequilibrium modes, and work done on electrons by the electric field induced by the electron pressure gradient [56]. The Joule heating source term represents the transfer of energy into vibrational modes through electron-molecule collisions. The constant $\gamma$ partitions the Joule heating between different nonequilibrium energy modes and varies from 0 to 1 depending on the reduced electric field. The reduced electric field is the magnitude of the electric field divided by the pressure [88] or by the total number density [89, 90]. Regardless of the reduced electric field definition, the pressure or total number density correlates with the mean free path of the electrons, while the electric field affects the electron mobility. These values help determine the electron energy distribution function which specifies the probability that an electron-molecule collision will result in energy deposition into a vibrational energy mode. The constant $\gamma$ is usually determined from reference tables, though it is possible to compute it directly from solutions to Boltzmann's equation, as discussed in Section 5.1. The final variable is the magnetic field vector $\mathbf{B}$.

In addition to the inclusion of Joule heating, accurate representation of the MHD effects requires the insertion of a magnetic (Lorentz) force, $\mathbf{j} \times \mathbf{B}$, in the momentum equation, as seen in Eqn. 4.3:

$$
\frac{\partial \rho \mathbf{u}}{\partial t}+\nabla \cdot(\rho \mathbf{u u}+p \mathbf{I}-\tau)=\mathbf{j} \times \mathbf{B}
$$

where $\rho$ is the density, $\mathbf{I}$ is the identity matrix, and $\mathbf{u u}$ is a $3 \times 3$ tensor containing all the products of the components of the velocity vector, as seen in Eqn. 4.4: 


$$
\mathbf{u} \mathbf{u}=\left[\begin{array}{ccc}
u^{2} & u v & u w \\
v u & v^{2} & v w \\
w u & w y & w^{2}
\end{array}\right]
$$

These additions to the standard conservation equations result in four previously undefined variables: $\gamma, \mathbf{j}, \mathbf{E}$, and $\mathbf{B}$, although $\gamma$ only needs to be defined for thermal nonequilibrium simulations, and can be estimated using several external approaches. The remaining variables are determined by assuming the Low Magnetic Reynolds Number Approximation described below.

\subsubsection{Low Magnetic Reynolds Number Approximation}

The three additional variables appearing in the conservation equations $\mathbf{j}, \mathbf{B}$, and E are determined by first considering the magnetic Reynolds number, as seen in Eqn. 4.5:

$$
R e_{m}=u L \mu_{0} \sigma
$$

where $L$ is the length scale, $\mu_{0}=4 \pi \times 10^{-7} \mathrm{~N} / \mathrm{A}^{2}$ is the permeability of free space, and $\sigma$ is the gas electrical conductivity. For example, Section 6.2 explores a MHDHeat Shield concept which has a hemispherical vehicle $(L \sim 0.1 \mathrm{~m})$, equipped with a strong electromagnet to partially deflect an incoming gas of weakly-ionized argon $\left(\sigma \sim 10^{3} \Omega^{-1} \mathrm{~m}^{-1}, u=3000 \mathrm{~m} / \mathrm{s}\right)$, which results in magnetic Reynolds number, $R e_{m} \sim 0.3$

The magnetic Reynolds number is a nondimensional variable that assesses how easily the magnetic field lines are transported by the fluid (advection), rather than through the fluid (diffusion). Because the magnetic Reynolds number is assumed 
to be small for the cases of interest, the magnetic field will diffuse or smooth out so its effects are determined by its boundary conditions. Consequently, the induced magnetic field can be neglected [91]. This means only an external applied magnetic field is present in the flow (and must be specified).

With the magnetic field specified for a steady state simulation, the current density and electric field vectors are related using a tensor form of the generalized Ohm's law, Eqn. 4.6:

$$
\mathbf{j}=\tilde{\sigma} \cdot(\mathbf{E}+\mathbf{u} \times \mathbf{B})
$$

where $\tilde{\sigma}$ is the electrical conductivity tensor, a compact way of accounting for ion-slip and the Hall effect [92]. Implementation and validation of the Hall effect is explained later in the chapter.

To solve Eqn. 4.6, the Ampère - Maxwell law, Eqn. 4.7, is simplified by assuming a negligible displacement current , $\epsilon_{0} \frac{\partial \mathbf{E}}{\partial t}<<\mathbf{j}$ :

$$
\nabla \times \mathbf{B}=\mu_{0} \mathbf{j}+\mu_{0} \epsilon_{0} \frac{\partial \mathbf{E}}{\partial t}
$$

where $\epsilon_{0}=8.85 \times 10^{-12} \mathrm{~F} / \mathrm{m}$ is the permittivity of free space. Neglecting the displacement current is a valid assumption when its magnitude is compared to the conduction current $\mathbf{j}=\sigma \mathbf{E}$ for typical conditions of air [93]. Applying the dot product to both sides of the reduced form of Eqn. 4.7, yields Eqn. 4.8 since $\mu_{0}$ is a constant:

$$
\nabla \cdot \mathbf{j}=0
$$

Assuming the electric field vector is smooth and rapidly decaying, Helmholtz's theorem is used to decompose it into irrotational and divergence-free component 
vector fields in Eqn. 4.9:

$$
\mathbf{E}=-\nabla \phi+\nabla \times \mathbf{A}
$$

where $\phi$ is the scalar potential and $\mathbf{A}$ is a vector potential, which should not be confused with the magnetic vector potential. The right side of Faraday's law of induction, Eqn. 4.10, must be zero for an externally applied magnetic field in a steady state simulation:

$$
\nabla \times \mathbf{E}=-\frac{\partial \mathbf{B}}{\partial t}
$$

Since $\nabla \times \mathbf{E}=0$, the electric field is only irrotational and the divergence-free term of Eqn. 4.9 must be zero, yielding Eqn. 4.11:

$$
\mathbf{E}=-\nabla \phi
$$

Combining Eqns. 4.6, 4.8, and 4.11 produces the equation for the electrical potential observed in Eqn. 4.12:

$$
\nabla \cdot \tilde{\sigma} \cdot[-\nabla \phi+\mathbf{u} \times \mathbf{B}]=0
$$

where $\tilde{\sigma}$ and $\mathbf{u}$ are defined by the flow solver, and $\mathbf{B}$ is specified as an input condition.

\subsubsection{Implementation}

To find the solution for $\phi$, and subsequently $\mathbf{E}$ and $\mathbf{j}$ using Eqns. 4.6 and 4.11, a finite-volume method is employed to be consistent with the flow solver. Rearranging the equation and integrating over an arbitrary volume yields Eqn. 4.13:

$$
\int_{V} \nabla \cdot[\tilde{\sigma} \cdot(\nabla \phi)] d V=\int_{V} \nabla \cdot[\tilde{\sigma} \cdot(\mathbf{u} \times \mathbf{B})] d V
$$


where $V$ is the volume. Equation 4.13 is further simplified by applying the Divergence Theorem [94], which states the divergence of an arbitrary volume is equal to its change (flux) through the surfaces. Introducing a scaling vector $\mathbf{C}$, which is defined in Eqn. 4.14, the equation is further simplified by combining the electrical conductivity tensor components, outward unit normal vector, and corresponding surface area (face), of each side of the arbitrary volume:

$$
\mathbf{C}=(\tilde{\sigma} \cdot \mathbf{n}) A
$$

where $\mathbf{n}$ is the outward facing unit normal vector for each face and $A$ is its corresponding area (not to be confused with the vector potential $\mathbf{A}$ ). In $3 \mathrm{D}$ : $\mathbf{C}=$ $\left[\left(\sigma_{1,1} n_{x}+\sigma_{1,2} n_{y}+\sigma_{1,3} n_{z}\right) A,\left(\sigma_{2,1} n_{x}+\sigma_{2,2} n_{y}+\sigma_{2,3} n_{z}\right) A,\left(\sigma_{3,1} n_{x}+\sigma_{3,2} n_{y}+\sigma_{3,3} n_{z}\right) A\right]$. Equating the surface integral to a sum over an arbitrary number of faces in a specific cell produces Eqn. 4.15:

$$
\sum^{\text {faces }}(\nabla \phi) \cdot \mathbf{C}=\sum^{\text {faces }}(\mathbf{u} \times \mathbf{B}) \cdot \mathbf{C}
$$

During every iteration of the flow solver, the MHD subroutine determines the electric and current field vectors by solving Eqn. 4.15 for $\phi$. With the electrical conductivity tensor and the velocity vector provided by the flow solver, and the magnetic field specified from the input conditions, the right side of Eqn. 4.15 is computed directly by approximating each face vector as a volumetric average of the adjoining cells. A nonuniform cell size, finite difference scheme determines the electric potential flux $\nabla \phi$ through each face as seen in Fig. 4.1, by relating the nonuniform cell spacing on the left and right sides of the face using a constant $\alpha$.

Following [95] and [54], Eqn. 4.16 provides a second order, nonuniform difference for the flux at the cell face: 


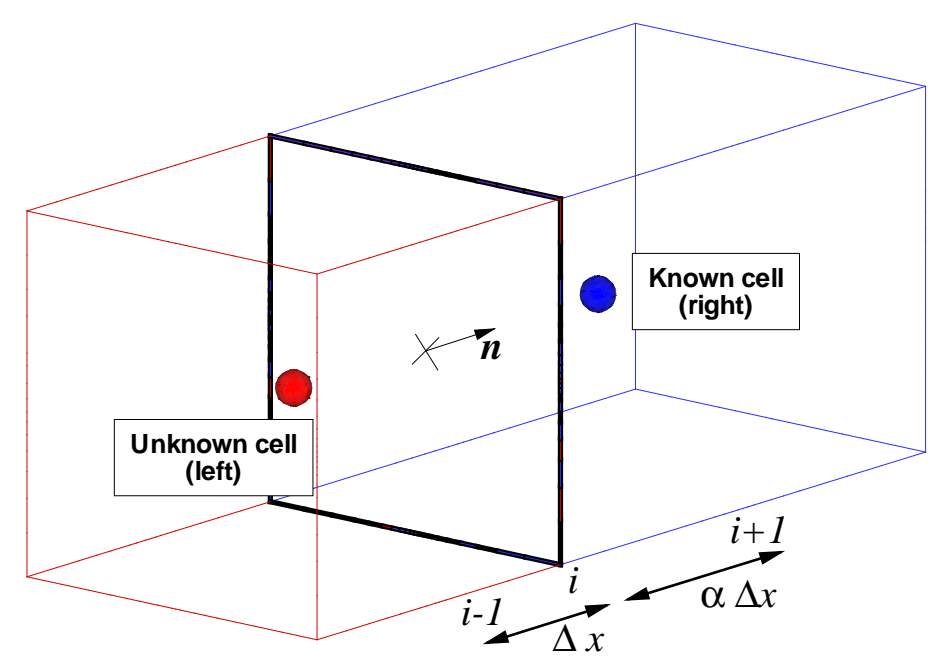

Figure 4.1: An illustration of the nonuniform finite difference methodology used to find the flux through face $i$ from $i-1$ to $i+1$. ( $\phi$ is known at $i+1$ [blue] and unknown at $i-1$ [red])

$$
\frac{\partial \phi}{\partial x}=\frac{\phi_{i+1}+\left(\alpha^{2}-1\right) \phi_{i}-\alpha^{2} \phi_{i-1}}{\alpha(\alpha+1) \Delta x}
$$

where the distance between the left cell center and the face center is $\Delta x=\left|x_{i}-x_{i-1}\right|$ and the ratio of the right and left distances $\alpha=\left|\frac{x_{i}-x_{i+1}}{x_{i}-x_{i-1}}\right|$.

Using Eqn. 4.16, Eqn. 4.15 is applied to all cells within the domain using the Successive Over-Relaxation SOR technique. The SOR method is an iterative, explicit solver that utilizes the directional change of the solution to extrapolate an improved solution based on a relaxation constant $\omega$. The method usually converges more slowly than an implicit scheme [54], but is easier to implement and parallelize. In order to reduce the number of iterations needed to achieve convergence $\omega>1$. However, if $\omega$ is set too high, the solver can become unstable. For all computations presented in the chapter $\omega \leq 1.70$. 


\subsubsection{MHD Boundary Conditions}

Although the solution of Eqn. 4.15 can be computed for all cells within the domain, the faces corresponding to the domain boundaries require special attention. For domains with a solid surface (e.g., a vehicle surface), mixed boundary conditions are generally employed. For electrodes that are good conductors, the electric potential is specified, either as a fixed value or determined by auxiliary equations representing an external circuit. For an insulated boundary, the normal component of current is set to zero: $\mathbf{j} \cdot \mathbf{n}=\tilde{\sigma} \cdot(\mathbf{E}+\mathbf{u} \times \mathbf{B}) \cdot \mathbf{n}=0$. This can be a complicated boundary condition in the general case of tensor conductivity, but in the case of scalar conductivity and no-slip wall conditions $(\mathbf{u}=0)$, it reduces to a vanishing normal electric field boundary condition $\mathbf{E} \cdot \mathbf{n}=0$.

The normal direction at a symmetry plane must have a zero electric field, $\mathbf{E} \cdot \mathbf{n}=0$. Because a finite-volume method is employed to solve the fluid conservation laws and Poisson equation, each boundary cell has an accompanying ghost cell. By definition, the volumetric centroid of the ghost cell lies on the outward unit normal vector of its adjoining real cell face, so the symmetry plane boundary condition is easily implemented as $\partial \phi / \partial n=0$.

The proper boundary conditions in the far-field are less clear for aerodynamic MHD problems. For high accuracy, it may be necessary to solve the current continuity equation on a larger domain than the fluid conservation laws, since the magnetic field can interact with the far-field. However, for most external flows, the electrical conductivity should decay to a negligible value far from the vehicle surface, so it is reasonable to set the normal component of the electric field to zero. The normal component of the electric field is also assumed to vanish at the inlet, $\mathbf{E} \cdot \mathbf{n}=0$. Finally, the outlet is assumed to be sufficiently far downstream of the primary MHD 
interaction that it is reasonable to set the normal component of the electric field to zero. Table 4.1 lists all domain boundaries and their respective conditions.

Table 4.1: Boundary conditions for the MHD solver

\begin{tabular}{ccc}
\hline \hline Location & Type & Condition \\
\hline Inlet & Neumann & $\mathbf{E} \cdot \mathbf{n}=0$ \\
Far-field & Neumann & $\mathbf{E} \cdot \mathbf{n}=0$ \\
Symmetry & Neumann & $\mathbf{E} \cdot \mathbf{n}=0$ \\
Outlet & Neumann & $\mathbf{E} \cdot \mathbf{n}=0$ \\
Wall (electrode) & Dirichlet & $\phi=$ specified \\
Wall (insulating) & Neumann & $\mathbf{j} \cdot \mathbf{n}=0$ \\
\hline \hline
\end{tabular}

Dirichlet conditions are imposed in the ghost cells adjoining the wall (electrode) boundary such that the wall face electric potential equals the specified value. Neumann boundary conditions require $\phi$ in the ghost cell to be determined iteratively in conjunction with the interior cells of the MHD solver domain to satisfy a zero gradient electric field assumption $\mathbf{E} \cdot \mathbf{n}=0$.

\subsection{Validation of the MHD Solver}

In this section, the development and implementation of the MHD solver and its boundary conditions are validated for two scenarios. In addition, the formal order of accuracy of the method is determined using Richardson extrapolation. Both validation cases repeat computational work conducted by Gaitonde and Poggie [92], that provides validation scenarios that are easy to implement and have known analytical solutions. 


\subsubsection{Analytical case with Dirichlet Boundary Conditions}

This validation exercise has been utilized previously by Wan et al. [48], and is selected because it is simple, has magnetic and velocity field vectors, and an analytical solution. The governing equation for the test problem is listed in Eqn. 4.17:

$$
\nabla^{2} \phi=x e^{z}
$$

Dirichlet (known) boundary conditions are assigned to the ghost cells accompanying each side of the domain based on Eqn. 4.17, $\phi=x e^{z}$. To simplify the exercise, the electrical conductivity tensor is set to unity, $\tilde{\sigma}=\mathbf{I}$, where $\mathbf{I}$ is the identity matrix, although its exact value does not affect the solution as long as it is constant. The magnetic and velocity field vectors are set as $\mathbf{B}=\left[0, \frac{1}{2} e^{z}, 0\right]$ and $\mathbf{u}=\left[x, 0, \frac{-1}{2} x^{2}\right]$, respectively.

Several grids are generated and employed, including a uniform rectilinear grid, a nonuniform rectilinear grid, and a rotated nonuniform rectilinear grid, as seen in Fig. 4.2. For the nonuniform grids, cell clustering occurs near the origin $(x, y, z)=$ 0 . Each side of the grid has a length equal to one. Both non-rotated grids lie within the domain $0 \leq x \leq 1,0 \leq y \leq 1,0 \leq z \leq 1$.

The formal order of accuracy is estimated using the Richardson extrapolation function in Tecplot 360@ [78]. This method determines the formal order of accuracy by extrapolating the results from a sequence of doubly refined grids to determine the exact solution. The computed results are compared to the exact solution to determine residual error which is plotted with the average cell size on a log-log scale. The slope of the resulting plot is equal to the order of accuracy of the method.

The grid sizes used for the case examined are: $10 \times 10 \times 10$ (coarse), $20 \times 20 \times 20$ 


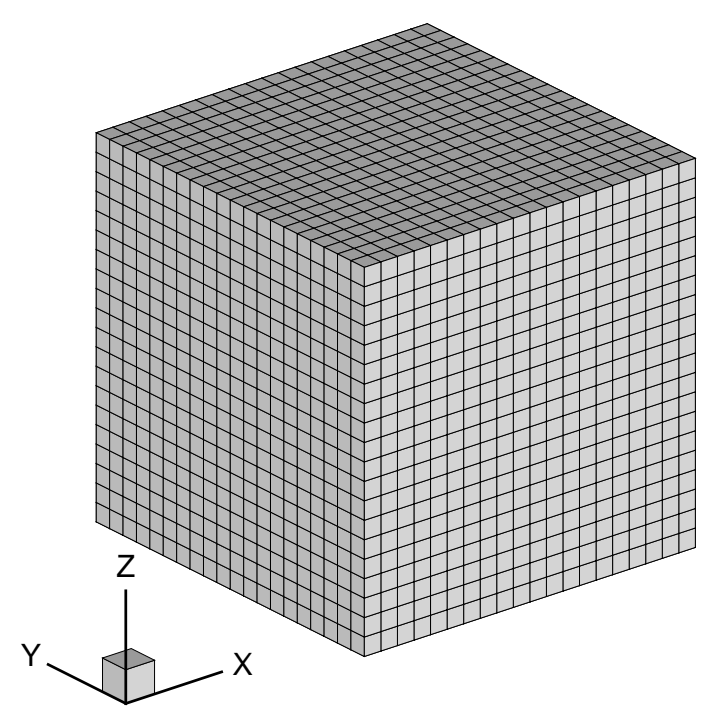

(a) Uniform

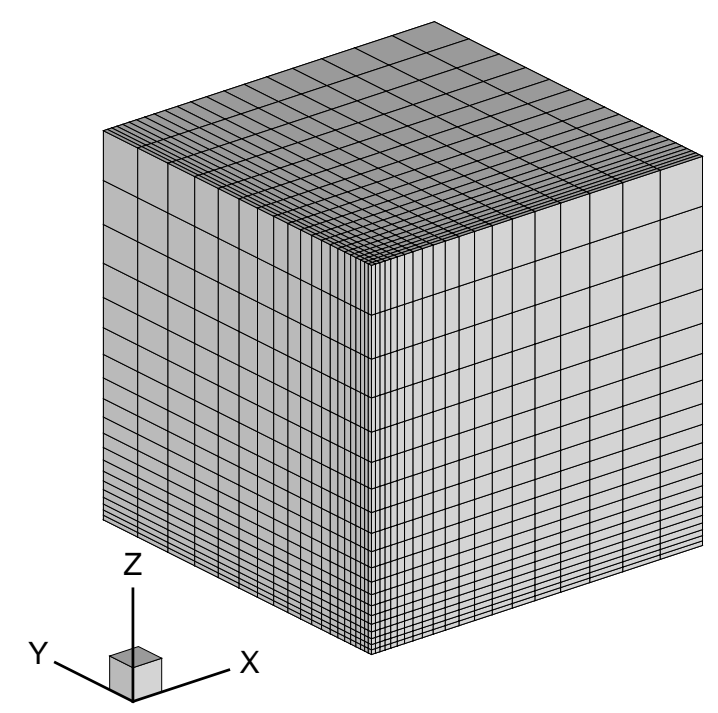

(b) Nonuniform

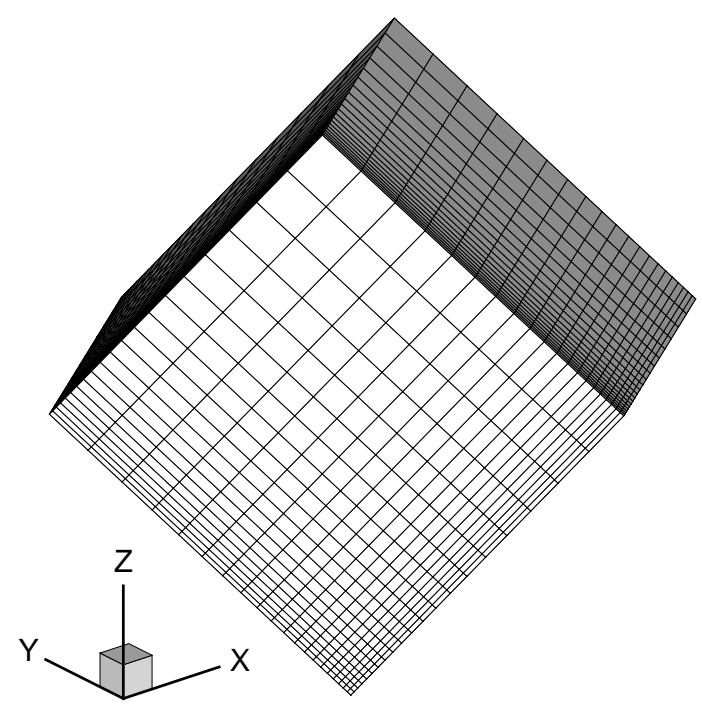

(c) Nonuniform (rotated $30^{\circ}$ along each axis)

Figure 4.2: Rectilinear grids used in the analytical MHD validation exercise. $(20 \times$ $20 \times 20)$ 
(medium), and $40 \times 40 \times 40$ (fine). The uniform mesh results in a formal order of accuracy of 1.999, which is expected since a second order accurate, finite difference scheme is employed. The nonlinear mesh produces a formal order of accuracy of 1.98, while the rotated mesh yields 1.80 due to the fact that each cell face normal vector does not align with the domain axes. As a result, the flux of each face $(\nabla \phi)$, is composed of nonzero derivatives in each axial direction, which lowers the overall accuracy since the finite difference flux scheme in Eqn. 4.16 is employed to determine the flux.

The accuracy of the solution is also observed by comparing the analytical solution to the computed solution. Figure 4.3 plots contours of the computed and analytical solutions for the rotated nonuniform mesh. As expected, the computed solution is very similar to the analytical solution.

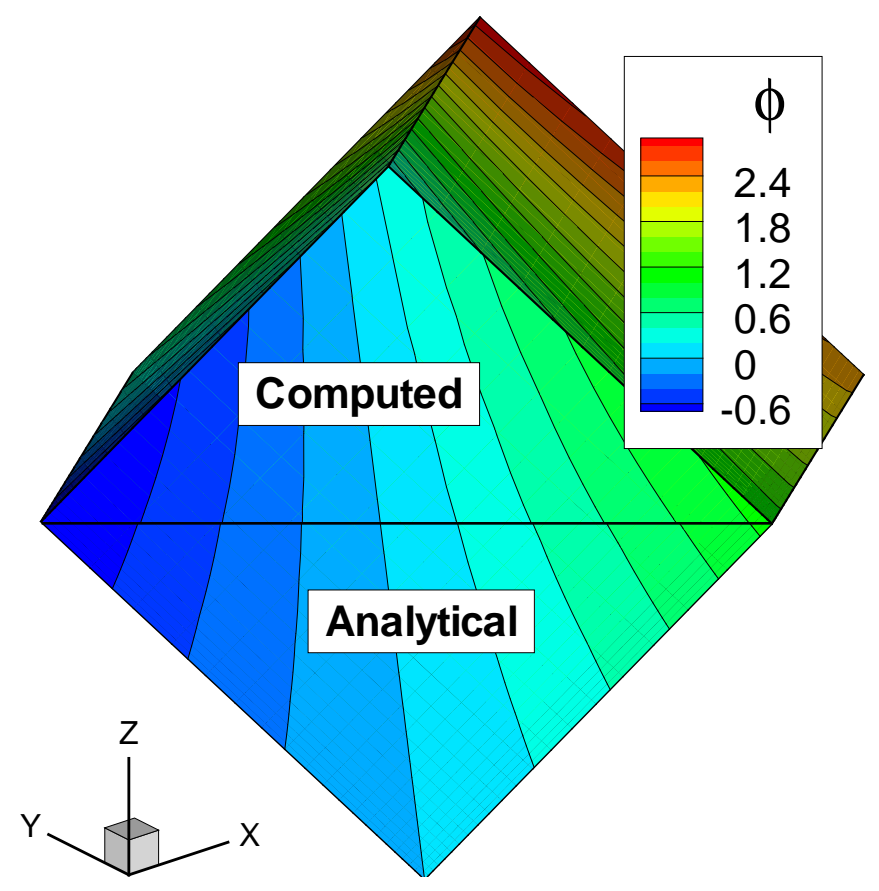

Figure 4.3: Contours of the electric potential $(\phi)$, for the analytical MHD validation case. The top half of the domain is populated by computed values while the bottom is theoretical. $(20 \times 20 \times 20)$ 


\subsubsection{Flow Between Two Electrodes}

A second validation exercise is performed, simulating current flow between two parallel electrodes separated by a distance of one meter along the $z$-axis. The electrodes have a specified potential such that the top electrode plate is equal to one volt and the bottom is set to zero. Neumann boundary conditions are employed along the remaining sides of the domain so the normal component of the gradient is zero, $\frac{\partial \phi}{\partial n}=0$. Figure 4.4 illustrates the domain with a rectilinear nonuniform mesh used in the simulation. Cell clustering is applied near both electrodes using a bi-exponential decay of cell size along the $z$ axis.

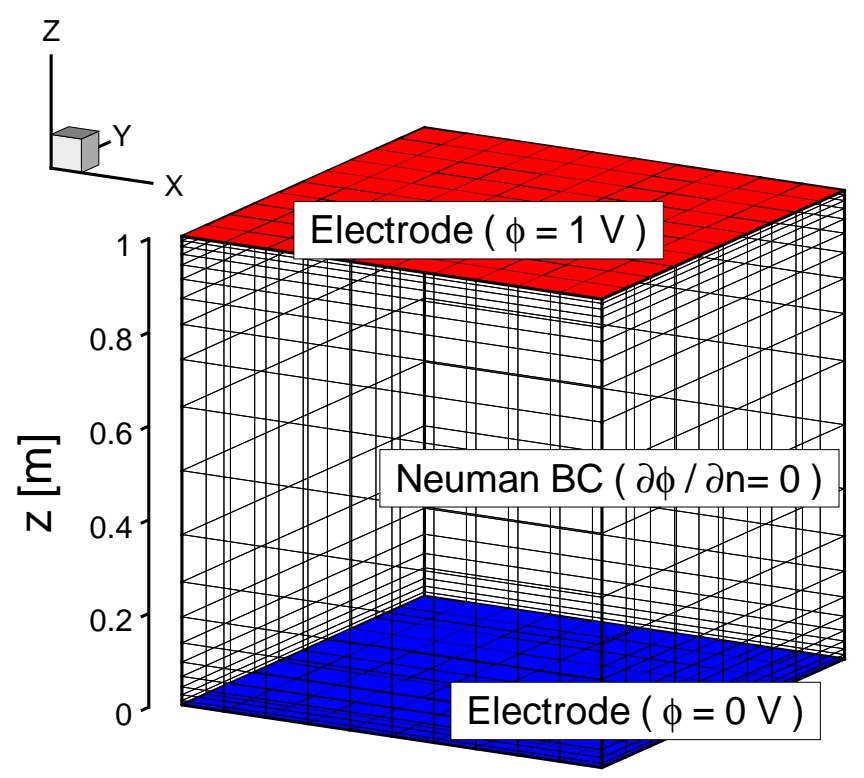

Figure 4.4: Nonuniform grid for current flow between parallel electrode plates. $(10 \times$ $10 \times 20)$

The simulations are computed assuming a zero velocity vector, $\mathbf{u}=0$, thereby simplifying Eqn. 4.12 to obtain a theoretical solution such that the current density is constant: $\mathbf{j}=-\tilde{\sigma} \cdot \nabla \phi=$ constant. Two different electrical conductivity models are employed. In the first case, the electrical conductivity is constant, $\sigma=1 \Omega^{-1} \mathrm{~m}^{-1}$, while the second case assumes the electrical conductivity diminishes as the distance 
from the bottom plate increases: $\sigma=1 / 2^{z} \Omega^{-1} \mathrm{~m}^{-1}$. Figure 4.5 plots the magnitude of the electrical conductivity versus position for each case.

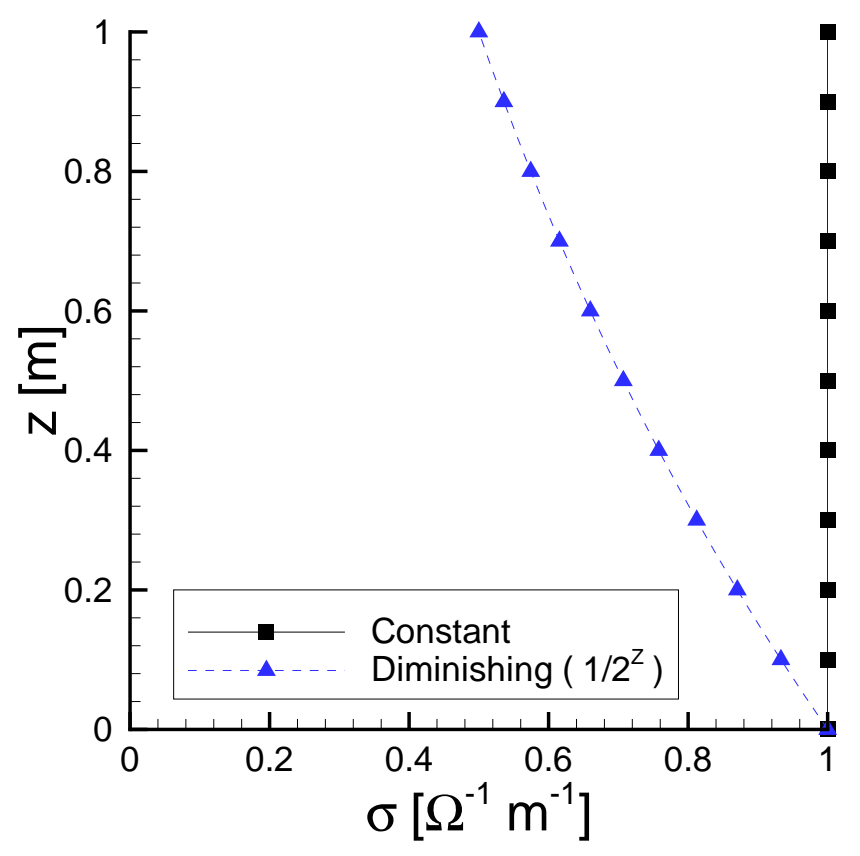

Figure 4.5: Various electrical conductivity models applied to flow between parallel electrode plates.

For constant electrical conductivity, $\sigma=1 \Omega^{-1} \mathrm{~m}^{-1}$, the gradient of the electric potential must be zero. The resulting electric potential is $\phi=z$ for the given boundary conditions. The theoretical solution for the second case is determined by recalling that Neumann boundary conditions are applied to the four other sides of the domain. This means $\phi$ will not vary in the $x$ or $y$ directions, $\mathrm{j}_{x}=\mathrm{j}_{y}=0$. Ignoring ionslip and Hall effects, the electrical conductivity tensor reverts to a scalar electrical conductivity $\sigma$, and allows a simple analytical solution to the current continuity equation to be found in Eqn. 4.18 by applying the domain's boundary conditions: 


$$
\begin{gathered}
\mathrm{j}_{z}=\sigma \frac{\partial \phi}{\partial z}=\left(1 / 2^{z}\right) \frac{\partial \phi}{\partial z}=\text { constant }=\mathrm{C} \\
\int_{0}^{1} \frac{\partial \phi}{\partial z} d z=\int_{0}^{1} \mathrm{C} 2^{z} d z \\
\frac{\partial \phi}{\partial z}=\ln 22^{z} \\
\phi=2^{z}+\text { constant }
\end{gathered}
$$

Using the boundary conditions specified at the each of the plates (i.e., the top electrode plate is equal to one volt and the bottom is set to zero), the constant in Eqn. 4.21 is determined to obtain the theoretical solution in Eqn. 4.22:

$$
\phi=2^{z}-1
$$

Figure 4.6 plots contours of the computed and analytical solutions for constant electrical conductivity on the nonuniform mesh, while Fig. 4.7 plots the electric potential distributions along the $x=0.5 \mathrm{~m}, y=0.5 \mathrm{~m}$ ray for both electrical conductivity cases. These figures demonstrate that the MHD solver accurately computes the electric potential for the validation cases performed, and verify the MHD module is successfully implemented.

\subsection{Parallel MHD Solver}

As with the flow solver, parallelization of the MHD routine is essential in order to provide solutions to the problems of interest in a reasonable amount of time by dividing the work among several processors. The parallel framework already existing in the flow solver is used to facilitate parallelization of the MHD routine. The flow

solver uses METIS [68] to partition the domain amongst the processors, and utilizes Message Passing Interface (MPI) calls to pass ghost cell properties between proces- 


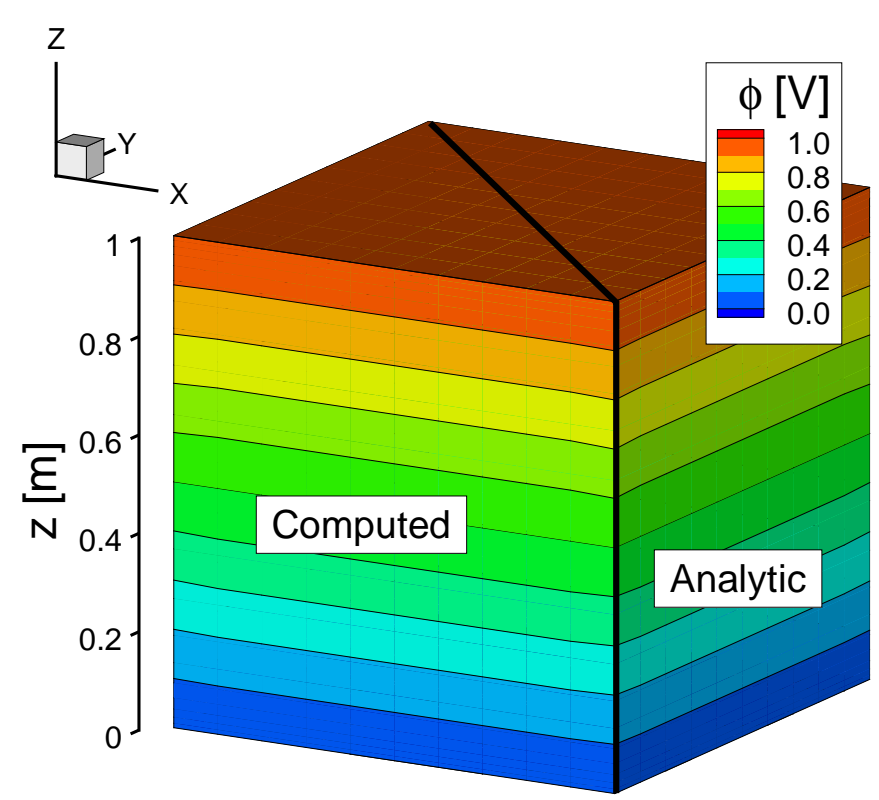

Figure 4.6: Contours of the electric potential $(\phi)$, for parallel electrodes with no flow $\left(\sigma=1 \Omega^{-1} \mathrm{~m}^{-1}\right)$. The left side of the domain is composed of computed values while the right side is theoretical. $(10 \times 10 \times 20)$

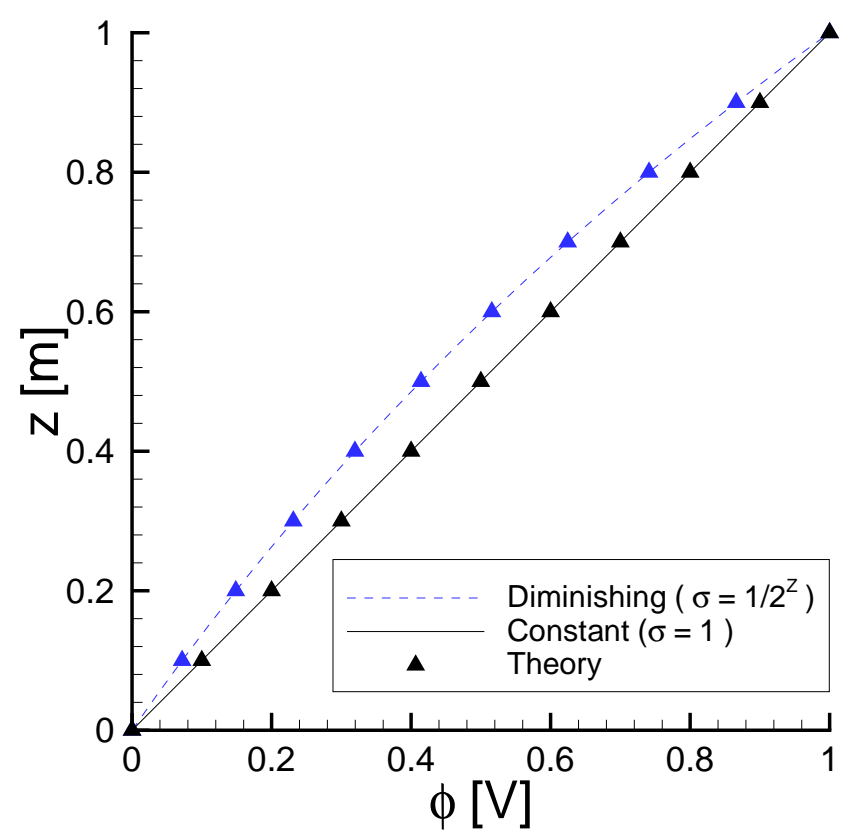

Figure 4.7: Electric potential distributions between parallel electrode plates with no flow for two electrical conductivity models. The distribution is extracted along the $x=0.5 \mathrm{~m}$ and $y=0.5 \mathrm{~m}$ ray. 
sors. By using the existing framework from the flow solver for creating partitions,

ghost cells, and their corresponding boundary cell links between partitions, computational overhead is reduced and parallel efficiency is increased. The drawback is that the MHD routine must use the same partitions, and subsequently, the same mesh as the flow solver. This can be problematic for specific geometries and conditions, especially when the grid resolution needed to obtain a grid-independent solution to Ohm's law is higher than that needed by the standard conservation equations (e.g., regions of the domain where the electromagnetic field, but not the flow-field, has large gradients). Nonetheless, this approach is still suitable for the present work because the computational cost per MHD cell is constant, and METIS partitions the mesh to minimize the number of boundary cells and equalize the number of cells per partition. This means the parallel efficiency of the MHD routine is consistent with the flow solver.

\subsubsection{Flow Between Parallel Electrodes}

Validation of the parallelized MHD module is accomplished by repeating the validation exercise described in Section 4.3.2, for the constant electrical conductivity scenario, $\sigma=1 \Omega^{-1} \mathrm{~m}^{-1}$. Figure 4.4 illustrates the setup and boundary conditions. As in Section 4.3.2, the theoretical solution reduces to a form where the electric potential $\phi=z$ for the given boundary conditions. Figure 4.8 plots the electric potential for the computed solution, obtained using four processors, and the theoretical solution. The computed solution matches the theoretical value and does not produce a discontinuity between partitions. This validation exercise verifies that information from each partition is successfully being shared with the other corresponding partitions, and that the MHD module is successfully parallelized. 


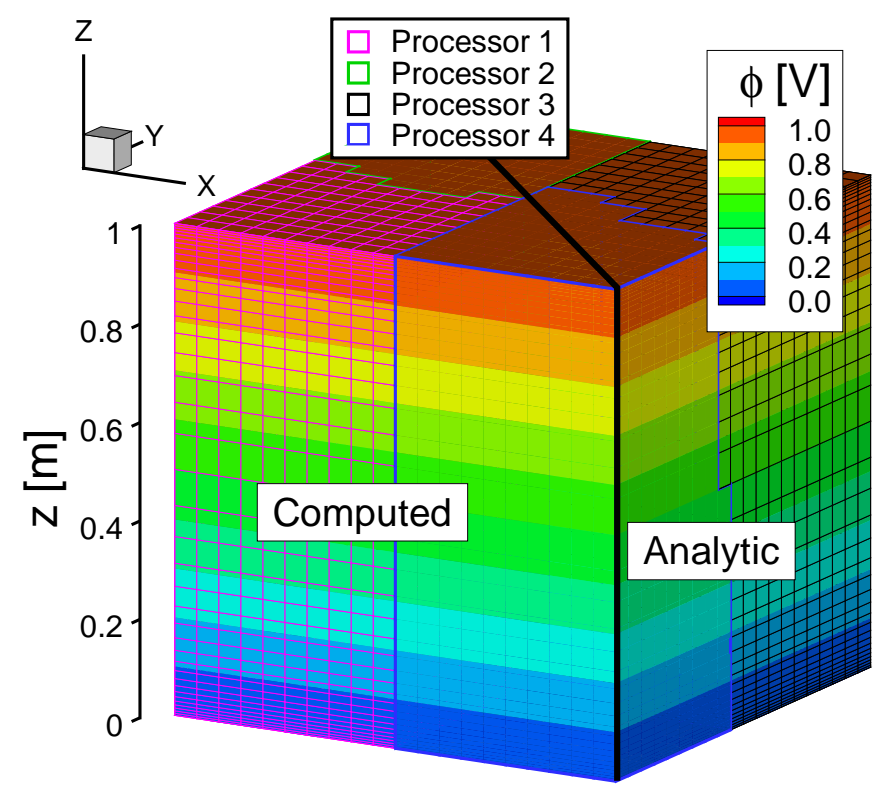

Figure 4.8: Electric potential contours between parallel electrode plates $(\sigma=$ $1 \Omega^{-1} \mathrm{~m}^{-1}$ and $\left.\mathbf{u}=0\right)$. The left side of the domain is composed of computed values using four processors, while the right side is the theoretical result. $(20 \times 20 \times 40)$

\subsubsection{Parallel Efficiency}

The parallel efficiency of the flow solver, with and without the MHD routine, is evaluated by simulating three-dimensional flow over a blunt leading edge, as seen in Fig. 4.9. The geometry has a length of $0.1 \mathrm{~m}$ and a vertical extent of $0.05 \mathrm{~m}$. The body geometry follows a power-law-shaped profile $\left(z=x^{0.5}\right)$, and has an aerodynamic performance similar to a blunt body [96]. The leading edge profile and freestream conditions selected result in the formation of a strong bow shock, which is beneficial for this investigation because the temperature increase due to the shock creates a small concentration of charged species in an otherwise neutral flow. The simulations are run using eleven species chemically reacting air $\left(\mathrm{N}_{2}, \mathrm{O}_{2}, \mathrm{NO}, \mathrm{N}, \mathrm{O}, \mathrm{N}_{2}^{+}, \mathrm{O}_{2}^{+}\right.$, $\mathrm{NO}^{+}, \mathrm{N}^{+}, \mathrm{O}^{+}$, and e), where (e) represents the electrons. The freestream conditions are consistent with air at $40 \mathrm{~km}$, as listed in Table 3.3 . 


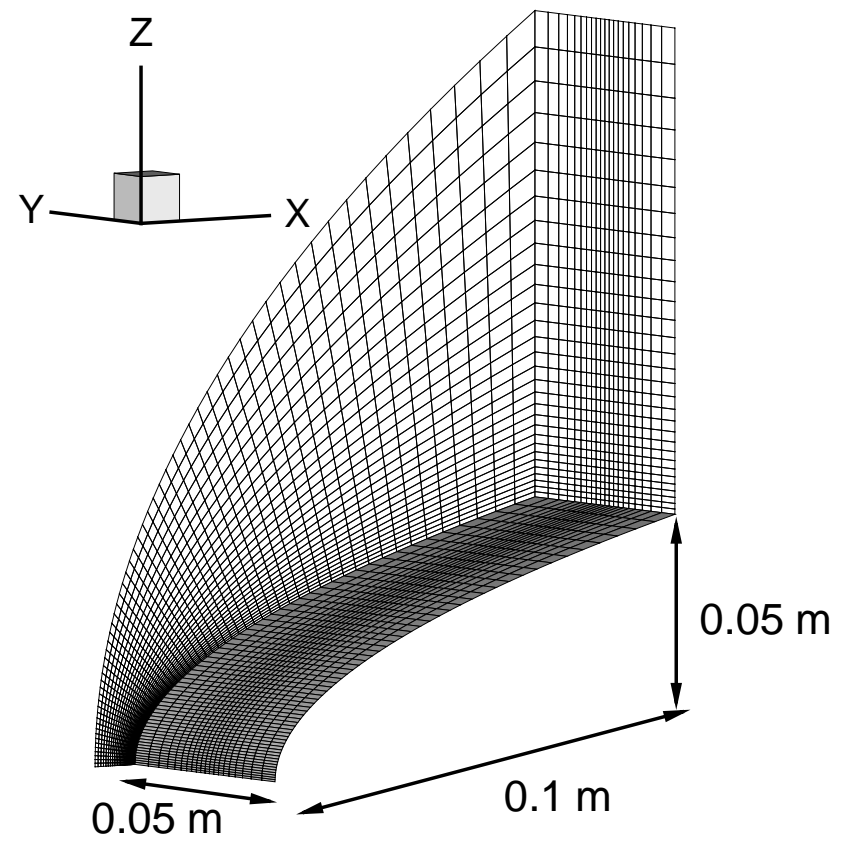

Figure 4.9: Geometry for a power-law shaped blunt-leading edge $z=x^{0.5} \cdot(60 \times 30 \times$ 20)

A grid independence study is performed on the geometry using the chemically reacting, nonequilibrium flow solver. A structured grid is generated because the present MHD routine cannot accommodate unstructured grids, and because a wellaligned structured mesh is known to produce better results in regions near the surface of the body and through a shock [72]. Exponential spacing is employed along each direction (along the body and radial from the body) so grid clustering occurs near the stagnation region. Exponential spacing places additional points exactly between existing points as the grid is doubly refined. The 'coarse' grid employs 30 points along the geometry, 15 points radial from the body, and 10 points along the width of the body $(30 \times 15 \times 10)$. The points along the width of the body are also clustered using exponential spacing so clustering occurs near the half-width. Additional grids double the points along each direction (medium: $60 \times 30 \times 20$, fine: $120 \times 60 \times 40$ ). A 'very fine' grid $(240 \times 120 \times 80)$, is also simulated because of differences observed 
in heat flux at the stagnation point between the 'medium' and 'fine' grid solutions. Figure 4.9 illustrates cell clustering for the 'medium' grid.

The pressure coefficient, Eqn. 2.7, and nondimensional heat flux, Eqn. 2.9, are computed along the wall centerline. Figure 4.10 plots the nondimensional heat flux and pressure coefficient for all four grids.

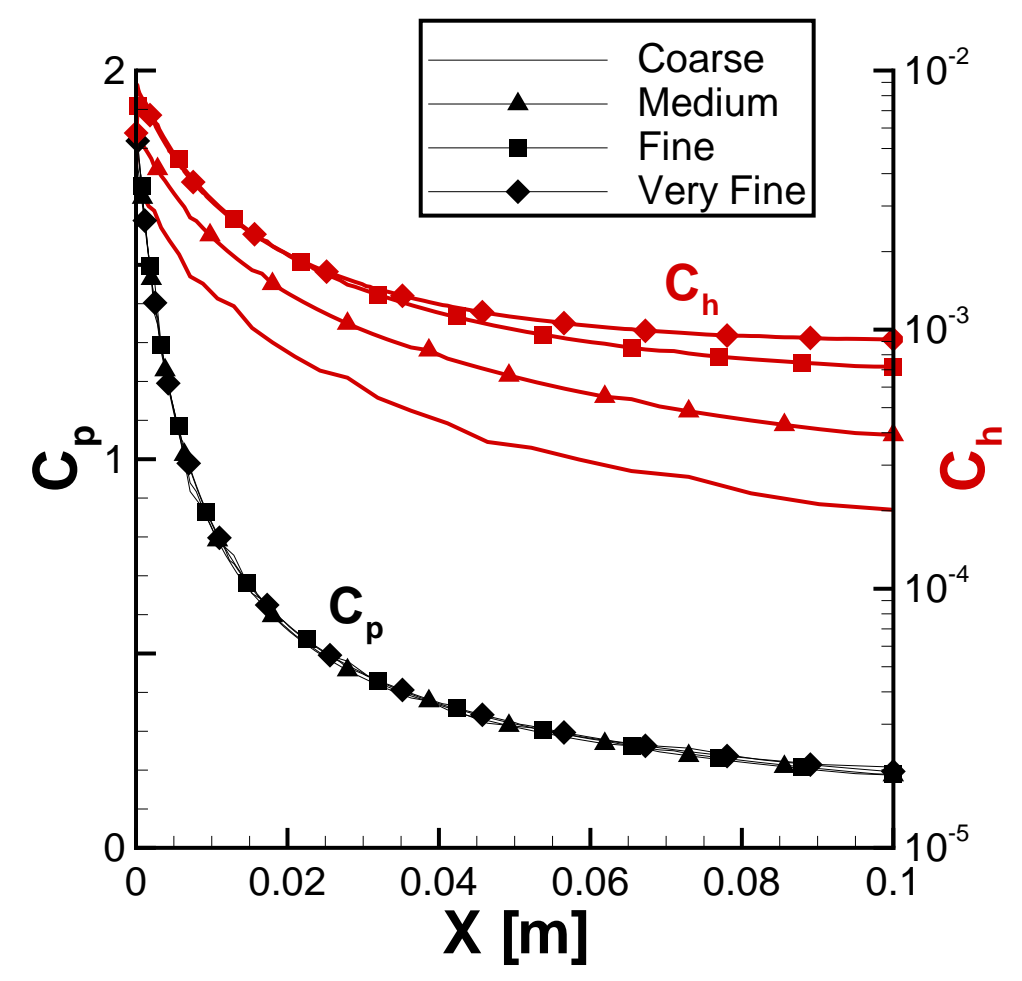

Figure 4.10: Coefficient of pressure and nondimensional heat flux along the center stagnation line $(y=0.025 \mathrm{~m})$, for Mach 12.6 air flow around a power-law blunt leading-edge for various grids.

The pressure coefficient is grid-independent for all the grids investigated. Excessively large cell spacing in the stagnation region of the 'coarse' grid creates a diffuse shock and increased shock standoff distance, as observed in Fig. 4.11, which plots temperature contours for the 'coarse' and 'fine' grids. Similarity between the 'fine' and 'very fine' grids for the nondimensional heat flux is found to be acceptable for the majority of the domain, so the 'fine' grid is assumed grid-independent. 


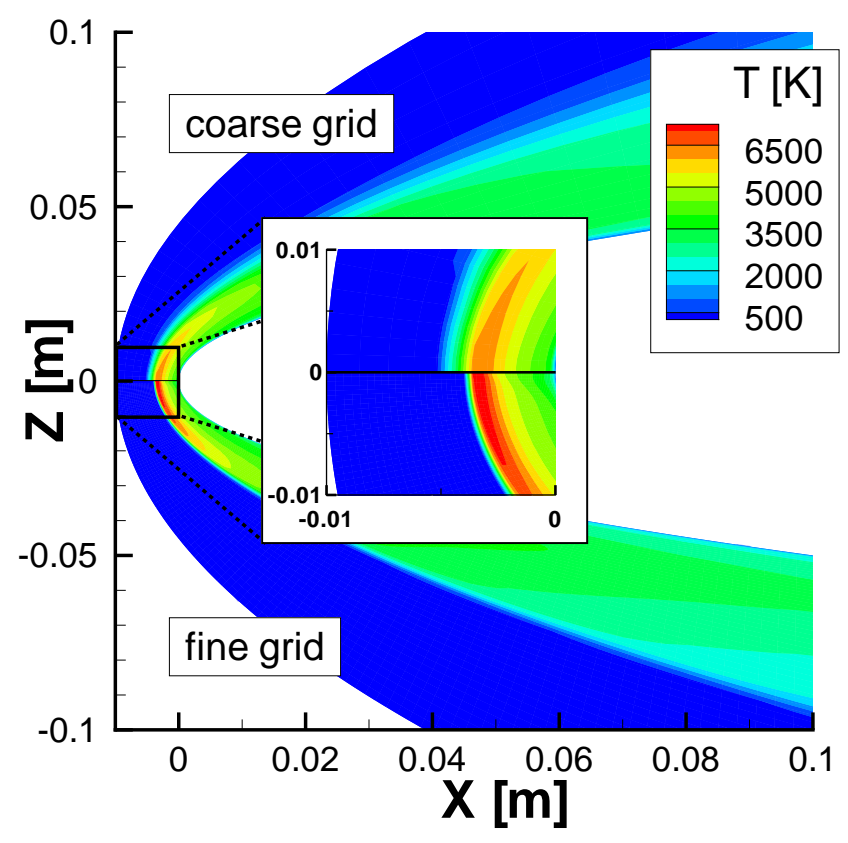

Figure 4.11: Temperature contours for Mach 12.6 air flow around a power-law blunt leading-edge from coarse $(30 \times 15 \times 10)$, and fine $(120 \times 60 \times 40)$, grids.

With a grid-independent mesh determined, parallel efficiency (speed up), is computed for the flow with and without the MHD routine. An arc discharge between an anode and cathode is simulated when the MHD routine is activated. Since the flow solver is run in thermal nonequilibrium, the constant $\gamma$ that partitions the Joule heating between different nonequilibrium energy modes is set to one because the focus of the simulations is to measure the computational cost of the MHD subroutine. The simulations are run for a specified number of iterations of the flow solver to determine the speed up as defined in Eqn. 4.23.

$$
\text { speed up }=\frac{\text { serial time }}{\text { parallel time }}
$$

where (parallel time) is the wall time for the simulation (total CPU-hours / number of processors). Figure 4.12 plots the speedup versus the number of processors. While the incremental cost due to running the MHD routine varies depending on 
the convergence criteria and relaxation constant, $\omega$, specified in the input routine, the routine does not appear to affect the overall parallel efficiency of the combined fluid-MHD code.

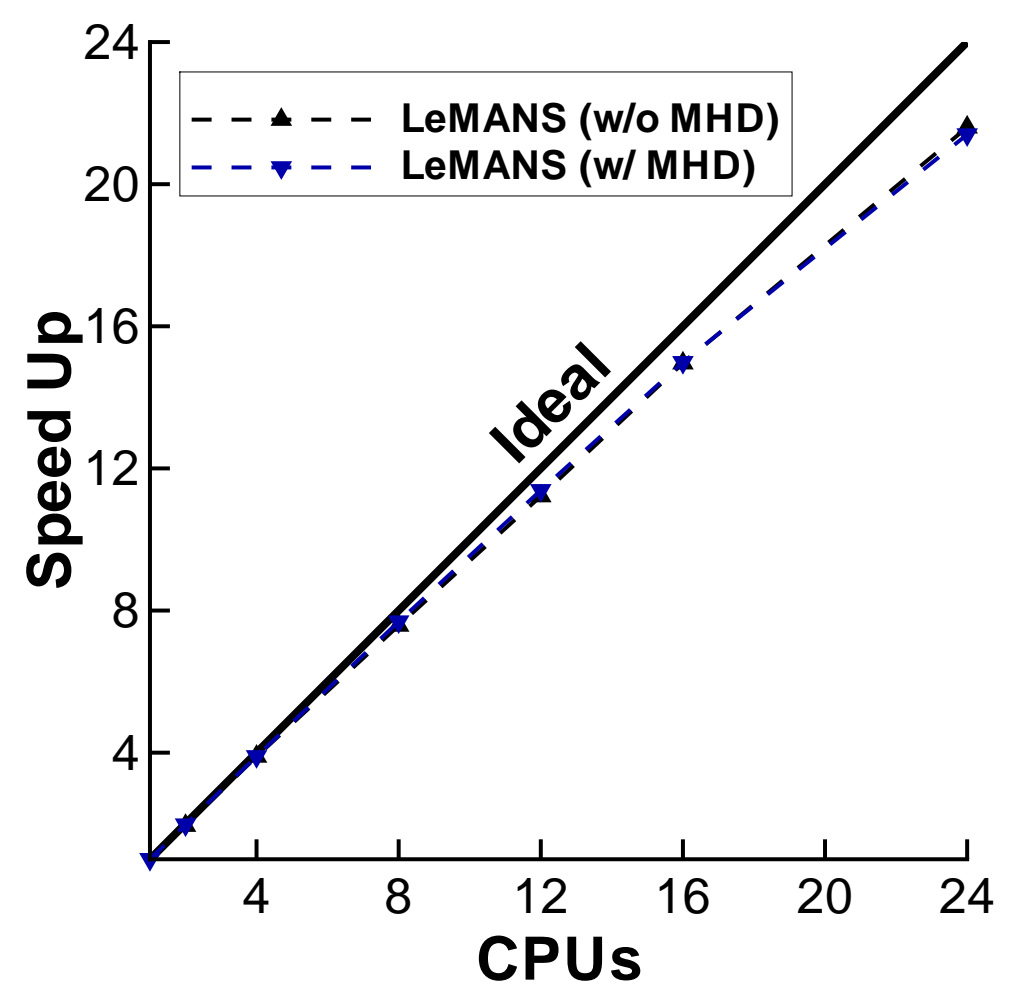

Figure 4.12: Speed up versus number of processors for flow solver with and without the MHD routine.

\subsection{Hall Effect}

As seen in Eqn. 4.6, the MHD subroutine incorporates the tensor nature of the electrical conductivity, following the formulation of Gaitonde and Poggie [97, 92]. This approach provides a compact way of accounting for ion-slip and the Hall effect. Equation 4.24 shows the electrical conductivity tensor with the Hall effect for Cartesian coordinates: 


$$
\tilde{\sigma}=\frac{\sigma}{D}\left[\begin{array}{ccc}
\mathrm{B}^{2}+\beta^{2} \mathrm{~B}_{x}^{2} & \beta\left(\beta \mathrm{B}_{x} \mathrm{~B}_{y}-\mathrm{B} \mathrm{B}_{z}\right) & \beta\left(\beta \mathrm{B}_{x} \mathrm{~B}_{z}+\mathrm{B} \mathrm{B}_{y}\right) \\
\beta\left(\beta \mathrm{B}_{y} \mathrm{~B}_{x}+\mathrm{B} \mathrm{B}_{z}\right) & \mathrm{B}^{2}+\beta^{2} \mathrm{~B}_{y}^{2} & \beta\left(\beta \mathrm{B}_{y} \mathrm{~B}_{z}-\mathrm{B} \mathrm{B}_{x}\right) \\
\beta\left(\beta \mathrm{B}_{z} \mathrm{~B}_{x}-\mathrm{B} \mathrm{B}_{y}\right) & \beta\left(\beta \mathrm{B}_{z} \mathrm{~B}_{y}+\mathrm{B} \mathrm{B}_{x}\right) & \mathrm{B}^{2}+\beta^{2} \mathrm{~B}_{z}^{2}
\end{array}\right]
$$

where the denominator $D=\mathrm{B}^{2}\left(1+\beta^{2}\right)$ and $\sigma$ is the electrical conductivity of the fluid. $\mathrm{B}_{x}, \mathrm{~B}_{y}$, and $\mathrm{B}_{z}$ are the components of the magnetic field vector and $\mathrm{B}$ is its magnitude. The Hall parameter $\beta$ is defined in Eqn. 4.25:

$$
\beta=\frac{e \mathrm{~B}}{m_{\mathrm{e}} \nu_{m}}
$$

where an elemental charge $e=1.6022 \times 10^{-19} \mathrm{C}$, the mass of an electron $m_{\mathrm{e}}=$ $9.11 \times 10^{-31} \mathrm{~kg}$, and $\nu_{m}$ is the electron-neutral particle momentum transfer collision frequency which is related to the electrical conductivity in Eqn. 4.26:

$$
\nu_{m}=\frac{e^{2} n_{\mathrm{e}}}{m_{\mathrm{e}} \sigma}
$$

where $n_{\mathrm{e}}$ is the electron number density. Equation 4.26 is combined with Eqn. 4.25 to yield Eqn. 4.27, which is directly computed from the fluid properties:

$$
\beta=\frac{\sigma \mathrm{B}}{e n_{\mathrm{e}}}
$$

\subsubsection{Segmented Electrodes}

Validation of the Hall effect is carried out by utilizing a computation study performed by Oliver and Mitchner [98]. In the experiment, finite segmented electrodes are infinitely repeated, aligned along the two walls of a channel, as seen in Fig. 


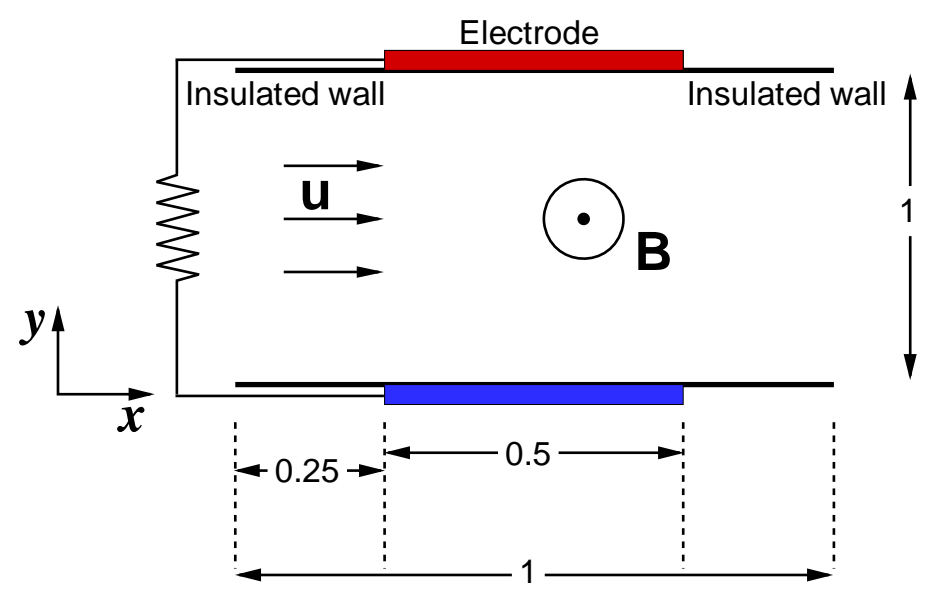

Figure 4.13: Schematic of the channel flow with finitely segmented electrodes. Units are in meters.

4.13. An externally applied magnetic field is positioned perpendicular to the channel velocity $\mathbf{u}$.

This exercise is inherently two-dimensional, but it is transformed into three dimensions by allowing the channel walls to be infinitely tall. Although this makes the problem computationally more expensive, the MHD routine is only suited for three-dimensional simulations. By using symmetric boundary conditions along the top and bottom planes of the domain, the actual height of the channel domain is set to a finite value of $0.1 \mathrm{~m}$ for the simulation, as seen in Fig. 4.14.

Because the channel is infinitely long, periodic boundary conditions are developed and employed at the domain inlet and outlet. The five point overlapping stencil shown in Fig. 4.15 transfers information between the periodic inlet and outlet planes. The scheme is selected because it is well suited for structured grids and is straight-forward to implement when the entire domain is situated on a single processor (serial). In this approach, a row of cells starts at the inlet and ends at the outlet. Along this row, the periodic boundary conditions state that the values in the first 


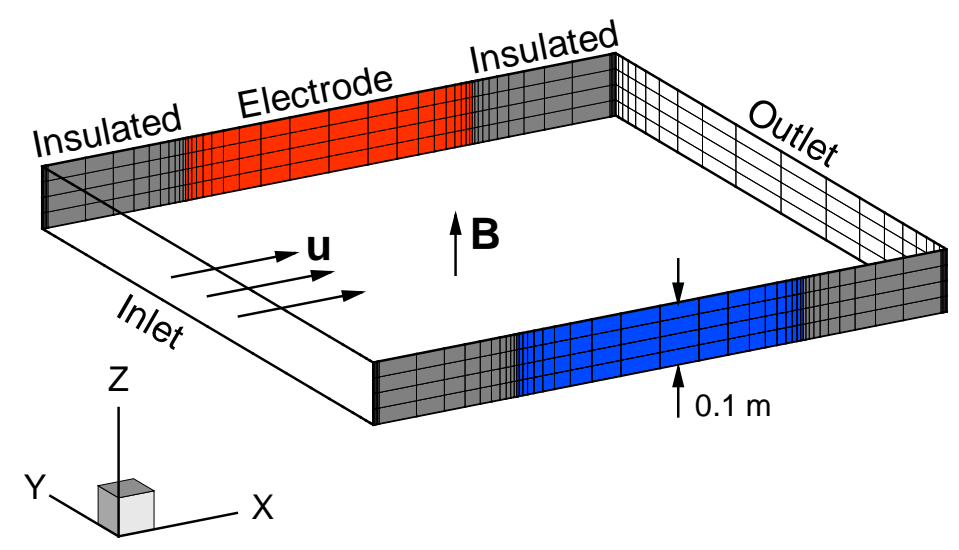

Figure 4.14: Geometry and boundary conditions for the 3D channel flow with finitely segmented electrodes. $(50 \times 20 \times 4)$

two cells nearest the inlet (the ghost cell and adjoining real cell), are set equal to the fifth and fourth cells from the outlet. These cells (the fifth and fourth cells from the outlet), along with the rest of the domain's interior cells, are determined by solving Eqn. 4.12 numerically. Likewise, the last two cells in the row (the last real cell and its adjoining ghost cell), are set equal to the values in the fourth and fifth cells from the inlet.

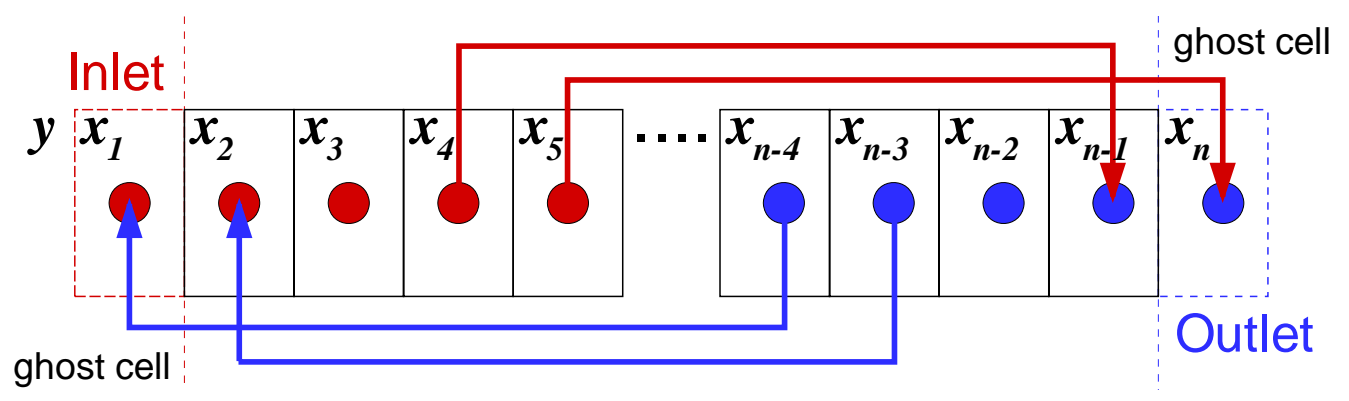

Figure 4.15: Cartoon of a five point stencil used for period boundary conditions.

Oliver and Mitchner carefully formulated this exercise so that the fluid velocity field did not affect the solution as long as $\nabla \times(\mathbf{u} \times \mathbf{B})=0$. During a single iteration of the flow solver, the MHD routine is executed assuming the velocity profile is only a function of the distance between the plates $\mathbf{u}=f(y)$ which satisfies $\nabla \times(\mathbf{u} \times \mathbf{B})=0$ 
as long as $\mathbf{B}=f(z)$. The velocity profile is assumed to be fully developed Poiseuille flow between parallel plates [65], as seen in Eqn. 4.28.

$$
\mathbf{u}=f(y)=u_{\max }\left(1-\frac{\left(y-y_{h}\right)^{2}}{h^{2}}\right)
$$

where $u_{\max }$ is the maximum velocity and is set to unity for this section, $u_{\max }=1 \mathrm{~m} / \mathrm{s}$. The $y$ location is measured from the center of the channel width $\left(y_{h}=0.5 \mathrm{~m}\right)$ and $h=0.5 \mathrm{~m}$ is the channel half-width.

A grid independence study is performed using non-reacting argon with a constant electrically conductivity $\sigma=1 \Omega^{-1} \mathrm{~m}^{-1}$. The channel walls are $1 \mathrm{~m}$ apart and the segmented electrodes are $0.5 \mathrm{~m}$ wide, with $0.5 \mathrm{~m}$ of insulated wall between them, so that the domain simulated has a length of $1 \mathrm{~m}$ in both the $x$ and $y$-directions. The channel walls are set to a height of $0.1 \mathrm{~m}$ in the $z$-direction with symmetric boundaries applied at the $z=0,0.1 \mathrm{~m}$ planes, effectively making the walls infinitely tall. Periodic boundaries are applied at the inlet and outlet, $x=0,1 \mathrm{~m}$, respectively. The grid utilizes exponential spacing along the wall surface such that additional points are positioned near the junction between the insulated wall and the electrode. Additionally, five even-spaced, points are located near both the inlet and outlet (along the wall surfaces) to maintain smooth periodic boundary conditions. Exponential spacing is also employed between the two walls such that cell clustering occurs near each surface. Uniform spacing is employed along the height of the wall ( $z$-direction), as seen in Fig. 4.14. The 'coarse' grid employs 50 points along the wall ( $x$-direction), 20 points between the walls ( $y$-direction), and 4 points in the $z$-direction. Two additional, doubly refined grids are also developed: $100 \times 40 \times 8$ (medium), $200 \times$ $80 \times 16$ (fine).

Grid independence is assessed by comparing solutions for the electric potential $\phi$ 
for the scenario without a magnetic field $\mathbf{B}=0$. Since the wall is infinitely tall, the solution in the $z$-direction is constant, and is only plotted along the $z=0 \mathrm{~m}$ plane. Extracting solutions of $\phi$ at two slices of the domain $x=0.25,0.5 \mathrm{~m}$, Figs. 4.16(a) and 4.16(b) show the potential does not vary significantly between the 'medium' and 'fine' grids, so the 'medium' solution is considered grid-independent and is employed in the rest of the section.

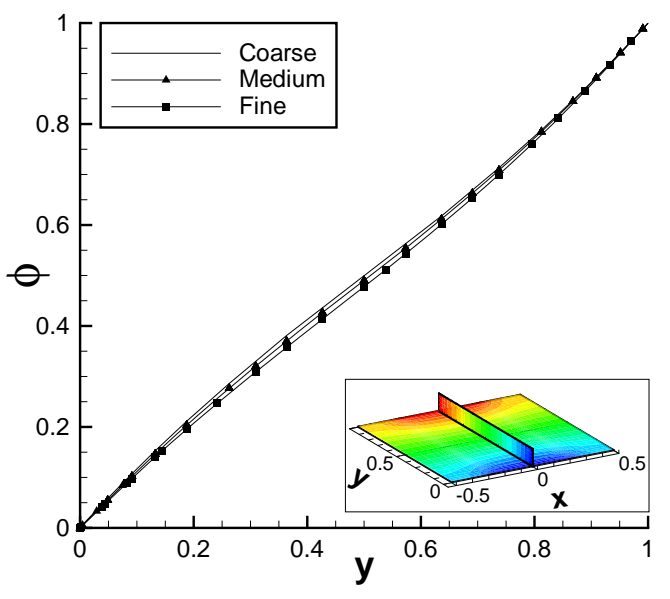

(a) $x=0 \mathrm{~m}$

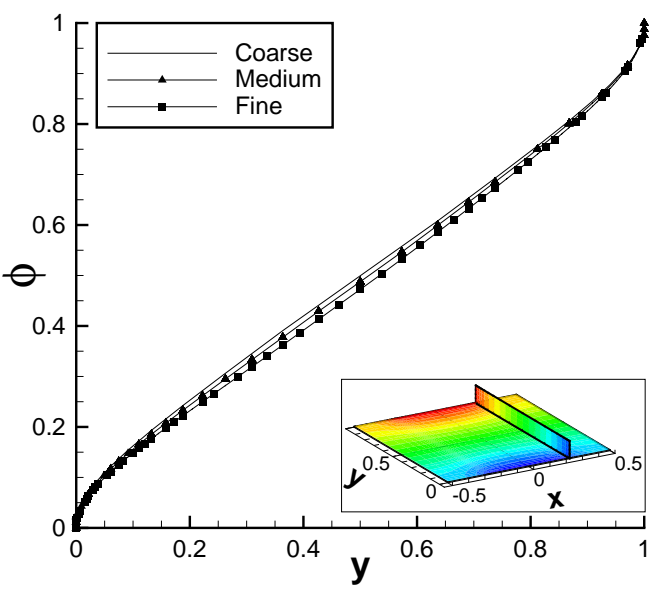

(b) $x=0.25 \mathrm{~m}$

Figure 4.16: Electric potential $(\phi)$, between two segmented electrodes at two different locations $(x=0 \mathrm{~m}$ and $0.25 \mathrm{~m})$, for various grids. $(z=0 \mathrm{~m})$

Without the presence of the magnetic field, the Hall effect has no significance, so the electrical conductivity tensor reverts to a scalar. The resulting electric potential solution is symmetric about the center of the electrode as seen in Fig. 4.17, where Fig. 4.17(a) is obtained by Gaitonde [97] and Fig. 4.17(b) is obtained using the 'medium' grid. Close examination of Figs. 4.17(a) and 4.17(b) show that the results obtained are consistent, further validating the MHD sub-routine.

To test the Hall effect, a second scenario is simulated. In this case, a nonzero magnetic field is applied externally. Consistent with the original analysis, the magnetic field is aligned with the $z$ axis, whereas the velocity is aligned with the $x$ axis. With- 


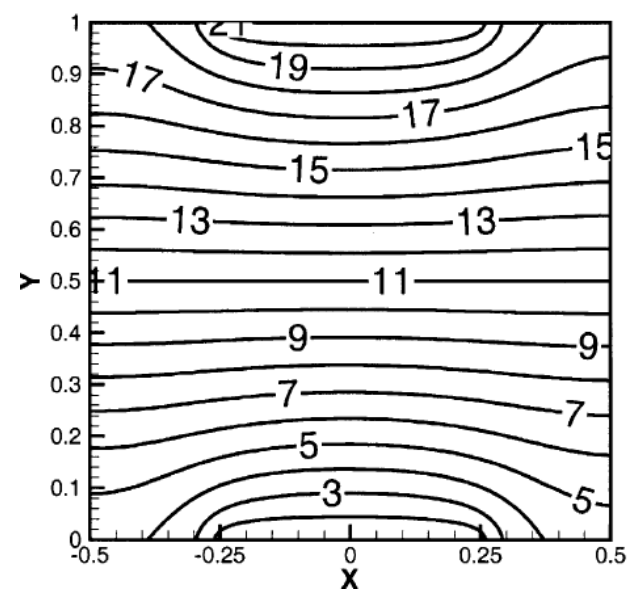

(a) From Gaitonde [97].

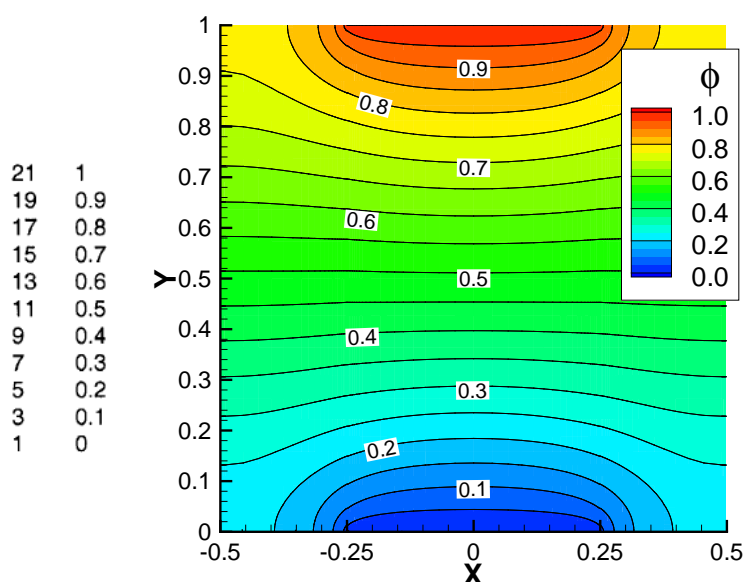

(b) Medium grid $(100 \times 40 \times 8)$ at $z=0$.

Figure 4.17: Electric potential contours for the segmented electrode channel without a magnetic field and constant electrical conductivity. $(\mathbf{B}=0, \sigma=$ $\left.1 \Omega^{-1} \mathrm{~m}^{-1}\right)$

out the Hall effect, a substantial current would only be created in the $y$ direction. With the Hall effect, magnetic terms also appear in the off-diagonal components of the electrical conductivity tensor seen in Eqn. 4.24. This results in the 'stretching' of the streamwise component of the current density vector, which becomes apparent by comparing the current density lines of Figs. 4.18(a) and 4.18(b). The results are also compared to the computational work by Gaitonde [97] in Fig. 4.18(c).

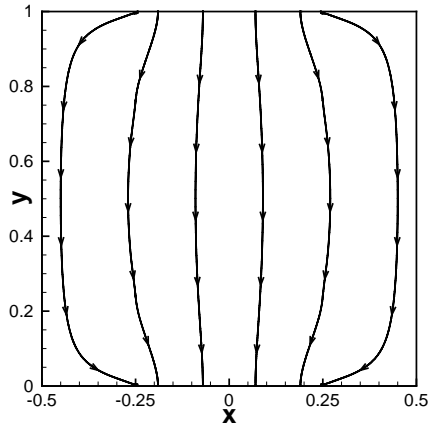

(a) Without the Hall effect

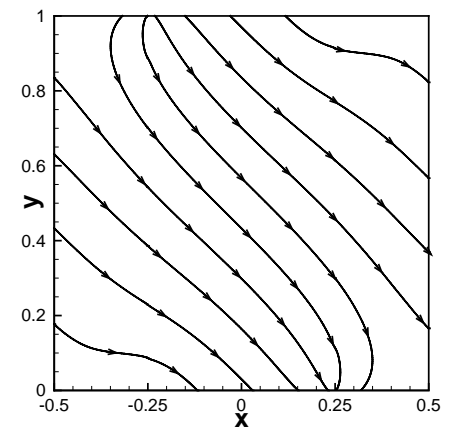

(b) With the Hall effect

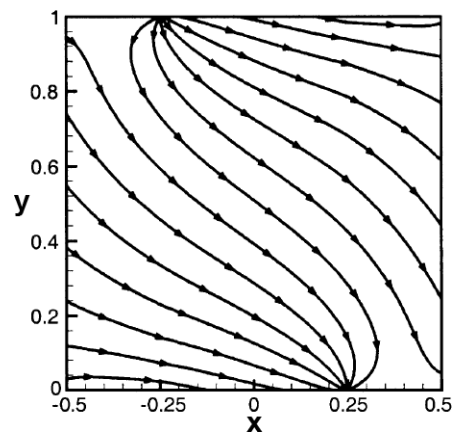

(c) From Gaitonde [97].

Figure 4.18: Current density streamlines $\mathbf{j}$ between two segmented electrodes with a magnetic field $\left(\mathrm{B}_{z}=1 \mathrm{~T}\right)$, constant electrical conductivity $(\sigma=$ $\left.1 \Omega^{-1} \mathrm{~m}^{-1}\right)$, and a streamwise velocity $(\mathbf{u}=f(y)) .(100 \times 40 \times 8)$ 
The results in Fig. 4.18(c) do not exactly match the results shown in Fig. 4.18(b) because Gaitonde employed a nondimensional MHD formulation, which results in additional coefficients in his electrical conductivity vector. Nonetheless, both figures portray similar characteristics, and indicate that the Hall effect is successfully implemented.

\subsection{Conclusions}

In this chapter, magnetohydrodynamic (MHD) effects, which arise due to the presence of the electric and magnetic fields, are accounted for within the flow solver by the addition of the magnetic force and Joule heating in the momentum and energy conservation equations. These new phenomena are the result of electromagnetic effects in the presence of a weakly-ionized gas, a product of strong shocks that form near hypersonic vehicles and some types of plasma-based flow control devices.

In addition to developing and iteratively coupling a three-dimensional MHD solver to the flow solver, the routine is parallelized using MPI such that the MHD subroutine is consistent with the overall parallel efficiency of the flow solver. Validation exercises for flow between two electrode plates and an analytic scenario demonstrated the routine and its boundary conditions are functioning correctly. In addition, the Hall effect is included in the MHD routine through the introduction of an electrical conductivity tensor, and is validated by computing flow between segmented electrodes. 


\section{CHAPTER V}

\section{Electrical Conductivity Modeling}

In Chapter IV, a three-dimensional magnetohydrodynamics (MHD) model is developed and coupled to a flow solver in order to accurately account for the electromagnetic effects in the flow. These effects are important in ionized flows, a condition typically observed in hypersonics because the high kinetic energy dissociates and ionizes the gas as it passes through a strong shock, or in regions where plasma-based flow control devices increase and/or utilize existing ionization. However, the method developed in Chapter IV requires an appropriate representation of the electrical conductivity of the plasma in order to accurately simulate these effects.

Most research in computational hypersonic MHD employs semi-empirical equations to represent the electrical conductivity because of the prohibitive computational cost associated with directly determining the electrical conductivity by solving Boltzmann's equation [99]. These semi-empirical equations are valid for a particular range of temperatures, pressures, and species composition. Although the use of semi-empirical equations to estimate the electrical conductivity is acceptable in some cases, it is not general, and is problematic when flow conditions exceed the range of the approximations employed.

This chapter explores several semi-empirical electrical conductivity models to 
illustrate their strengths and deficiencies. Surrogate modeling methods are used to develop a general approach for modeling solutions to Boltzmann's equation. These solutions are computed using a Boltzmann solver which employs an extensive list of accurate collision cross-section data to determine the electrical conductivity of a gas. In addition, simulations are presented for a hypersonic MHD-Heat Shield concept, which uses a magnetic field to reduce heat transfer to the vehicle by creating a magnetic force that opposes the incoming gas. These simulations are performed using several electrical conductivity models to emphasize the importance of using an appropriate model.

\subsection{Boltzmann Solver}

The semi-empirical models explored later in the chapter are compared with solutions of Boltzmann's equation found using a continuum Boltzmann solver developed by Kushner et al. [100], which is functionally equivalent to that proposed by Rockwood [101, 102]. Although the solver requires the translational temperature, pressure, and species mole fractions as input parameters, the solutions obtained only depend on the normalized electric field, $\mathrm{E} / N$, and each species mole fraction, $\chi_{s}$. The normalized electric field is the magnitude of the electric field $E=|\mathbf{E}|$, divided by the total gas number density, $N$, while the species mole fraction is its number density divided by the total number density, $\chi_{s}=n_{s} / N$. In order to maintain charge neutrality (consistent with the MHD approximation), the mole fraction of electrons is set to balance the charge density of the heavy particles.

A solution is achieved by converting the time dependent Boltzmann equation into $K$ coupled ordinary differential equations (ODE) by dividing the electron energy spectrum into $K$ energy bins [101]. The resulting matrix is integrated in time 
until the electron energy distribution function achieves a steady state. Following each time step, the matrix is updated to account for changes in the rate of electronelectron collisions due to changes in the distribution function. The collision frequencies required to compute the electrical conductivity are obtained by convolving the electron energy distribution functions derived by solving Boltzmann's equation with the mole-fraction weighted momentum transfer cross-sections for each species [103]. The cross-section data are taken from the compilations discussed in [104] and [105].

When the degree of ionization is small, $\alpha=\Sigma n_{\text {ions }} / N \ll 10^{-9}$, it is safe to assume that electron-electron collisions are unimportant, and they are ignored in Boltzmann's equation. This results in fairly sparse and banded matrices which are computationally inexpensive to invert and solve. However, as the degree of ionization becomes larger, electron-electron collisions become important and must be included in Boltzmann's equation. The electron-electron collisions tend to drive the electron energy distribution function toward a Maxwellian distribution as their collision rates increase. Although a Maxwellian distribution has a simple analytical form [99], the ionization may not be sufficiently high to assume the Maxwellian distribution. As a result, the Boltzmann solver must still invert the matrices, a task which is computationally expensive as the matrices are now fully populated due to the additional collision probabilities.

The accuracy of ignoring electron-electron collisions when $\alpha \ll 10^{-9}$ is examined in Fig. 5.1, which shows the average electron temperature $T_{e}$ versus the normalized electric field $\mathrm{E} / \mathrm{N}$ for a range of $\alpha$ in 7 species air $\left(\mathrm{N}_{2}, \mathrm{O}_{2}, \mathrm{NO}, \mathrm{N}, \mathrm{O}, \mathrm{NO}^{+}\right.$, and e). Note that $\mathrm{NO}^{+}$is the only ion, so $\alpha=\chi_{\mathrm{NO}^{+}}$. The neutral species mole fractions are arbitrarily set and kept constant $\left(\chi_{\mathrm{N}_{2}}=0.7487, \chi_{\mathrm{O}_{2}}=0.1643, \chi_{\mathrm{NO}}=1.048 \times 10^{-2}\right.$, $\left.\chi_{\mathrm{N}}=8.746 \times 10^{-3}, \chi_{\mathrm{O}}=6.67 \times 10^{-2}\right)$, whereas the lone ion concentration is adjusted 
to match the degree of ionization $\left(\chi_{\mathrm{NO}^{+}}=\alpha\right)$. Since $10^{-9} \leq \alpha \leq 10^{-3}$ in Fig. 5.1, the neutral species mole fractions do change slightly for each specified $\alpha$, but the minute adjustments do not noticeably influence the results and are ignored.

Figure. 5.1(a) shows that the electron temperature is a unique function of $\mathrm{E} / N$ for $\alpha \leq 10^{-7}$ (no electron-electron collisions). This is not the case when the electronelectron collisions are allowed, as is seen in Fig. 5.1(b) for $\alpha=10^{-7}, 10^{-8}, 10^{-9}$ curves. In addition, the curves representing a higher degree of ionization $\left(\alpha \geq 10^{-7}\right)$, experience a lower electron temperature as $\mathrm{E} / N$ approaches zero, as seen in a comparison of Figs. 5.1(a) and 5.1(b). Since the electron temperature is directly related to the electron mobility and, consequently, the electrical conductivity, accurately accounting for electron-electron collisions is important, especially when the degree of ionization is high.

Once a solution is achieved, the Boltzmann solver outputs several useful parameters including the equilibrium electron mobility $\mu_{\mathrm{e}}$, effective electron temperature $\mathrm{T}_{\mathrm{e}}$, and a fraction breakdown of each collision type (i.e., $\mathrm{N}_{2}$ elastic, $\mathrm{N}_{2}$ rotational, $\mathrm{N}_{2}$ vibrational state 1 , etc.), for a range of $\mathrm{E} / N$. The fractional breakdown of each collision type can be used to determine the amount of Joule heating that goes directly into the vibrational energy mode, and thus determine the constant $\gamma$ from Section 4.2 .

Equation 5.1 states the definition of electrical conductivity for a DC current:

$$
\sigma=\frac{e^{2} n_{\mathrm{e}}}{m_{\mathrm{e}} \nu_{m}}
$$

where $\sigma$ is the electrical conductivity, the elemental charge is $e=1.6022 \times 10^{-19} \mathrm{C}$, $n_{\mathrm{e}}$ is the electron number density, the electron mass is $m_{\mathrm{e}}=9.11 \times 10^{-31} \mathrm{~kg}$, and $\nu_{m}$ is the electron momentum transfer collision frequency. This is combined with the 


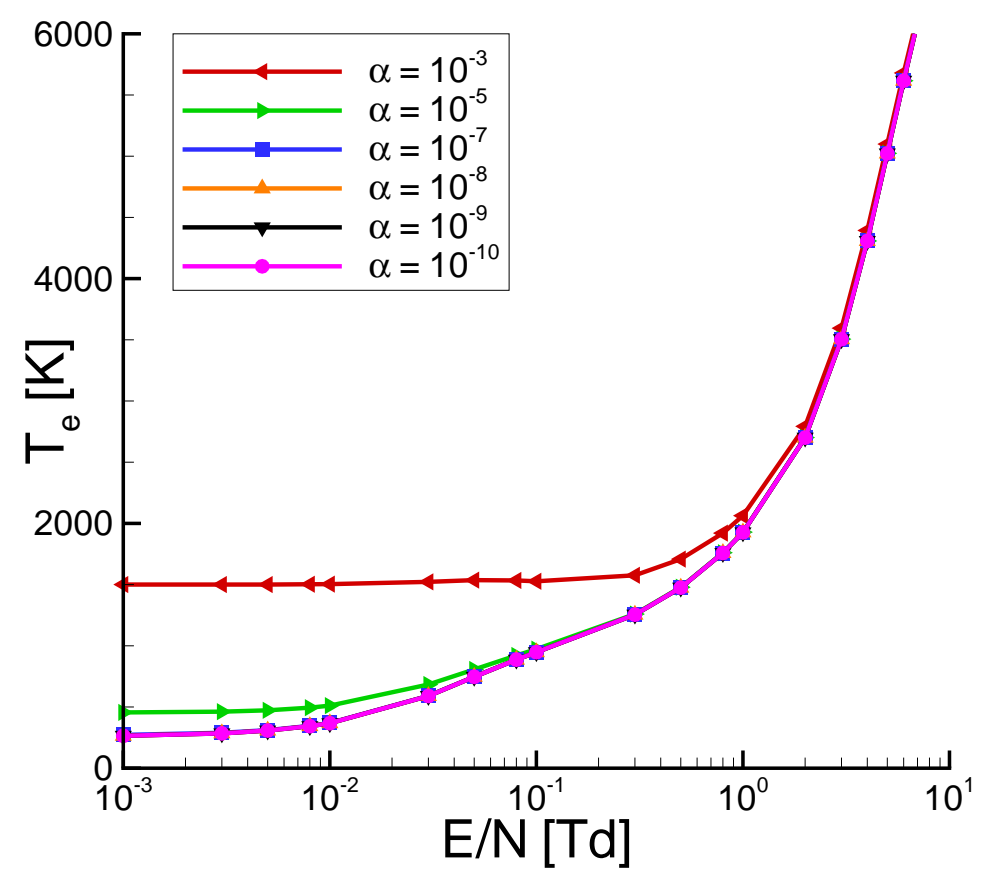

(a) Without e-e collisions.

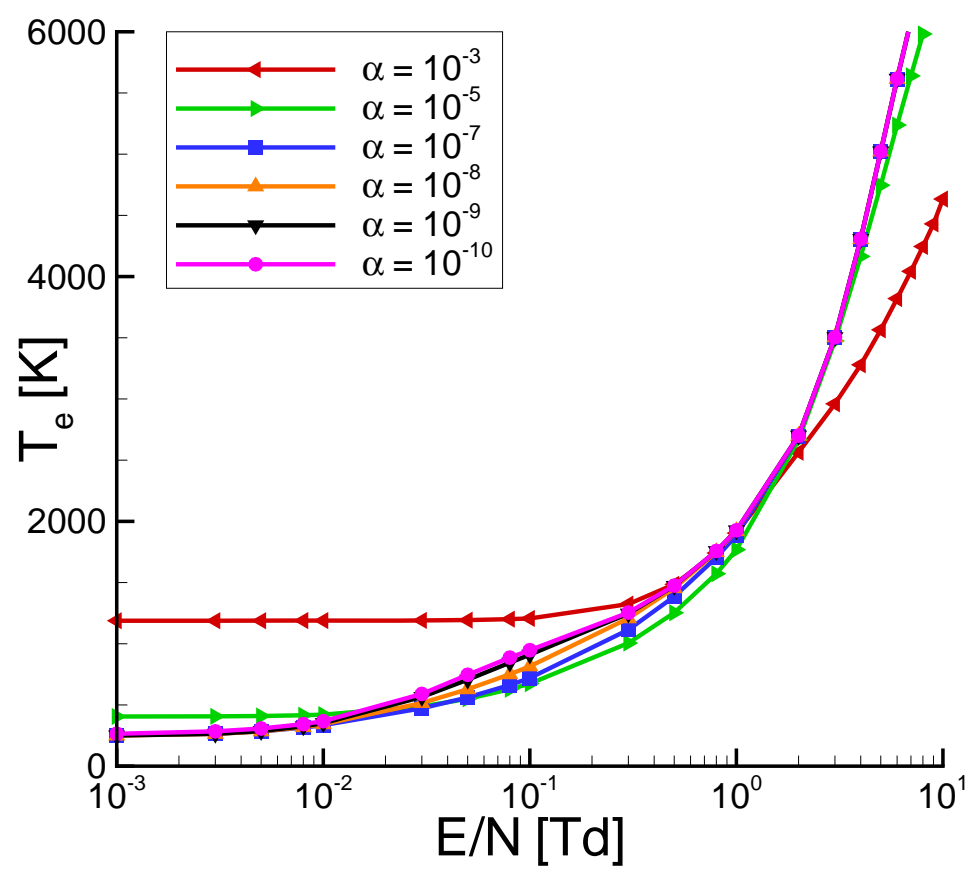

(b) With e-e collisions.

Figure 5.1: Electron temperature versus the normalized electric field for various degrees of ionization in 7 species air $\left(\mathrm{N}_{2}, \mathrm{O}_{2}, \mathrm{NO}, \mathrm{N}, \mathrm{O}, \mathrm{NO}^{+}\right.$, and e). 
definition of electron mobility, Eqn. 5.2, to yield Eqn. 5.3:

$$
\begin{gathered}
\mu_{\mathrm{e}}=\frac{e}{m_{\mathrm{e}} \nu_{m}} \\
\sigma=\mu_{\mathrm{e}} e n_{\mathrm{e}}
\end{gathered}
$$

where $\mu_{\mathrm{e}}$ is the electron mobility as determined by the solution from the Boltzmann solver. The Boltzmann solver outputs the electron mobility at standard atmosphere conditions $\left(p=1 \mathrm{~atm}, \mathrm{~T}=273 \mathrm{~K}, N=2.688 \times 10^{25} \mathrm{~m}^{-3}\right)$, so Eqn. 5.3 is rewritten for these outputs:

$$
\sigma=\mu_{0} N_{0} \text { e } \alpha
$$

where the reference electron mobility, $\mu_{0}$, and total number density, $N_{0}$, are the parameters output from the Boltzmann solver and $\alpha=n_{\mathrm{e}} / N=\Sigma n_{\text {ions }} / N$ is the degree of ionization because of local charge neutrality.

\subsection{Electrical Conductivity of Air}

As this research area has matured, several models have been developed to capture the behavior of the electrical conductivity, $\sigma$, which appears to be a function of pressure, temperature, and species composition, as seen in Fig. 5.2. Some empirical models only attempt to capture the conductivity once it starts to approach a horizontal asymptote (high temperature), because the conductivity is assumed negligible at lower temperatures. These models may also incorporate cutoff parameters to adjust their predictions in regions where the model performs very poorly. While these simplifications allow the model to be represented using only a few dimensional 
parameters (usually temperature and/or electron number density), the approach is not general and may be highly dependent on reference parameters.

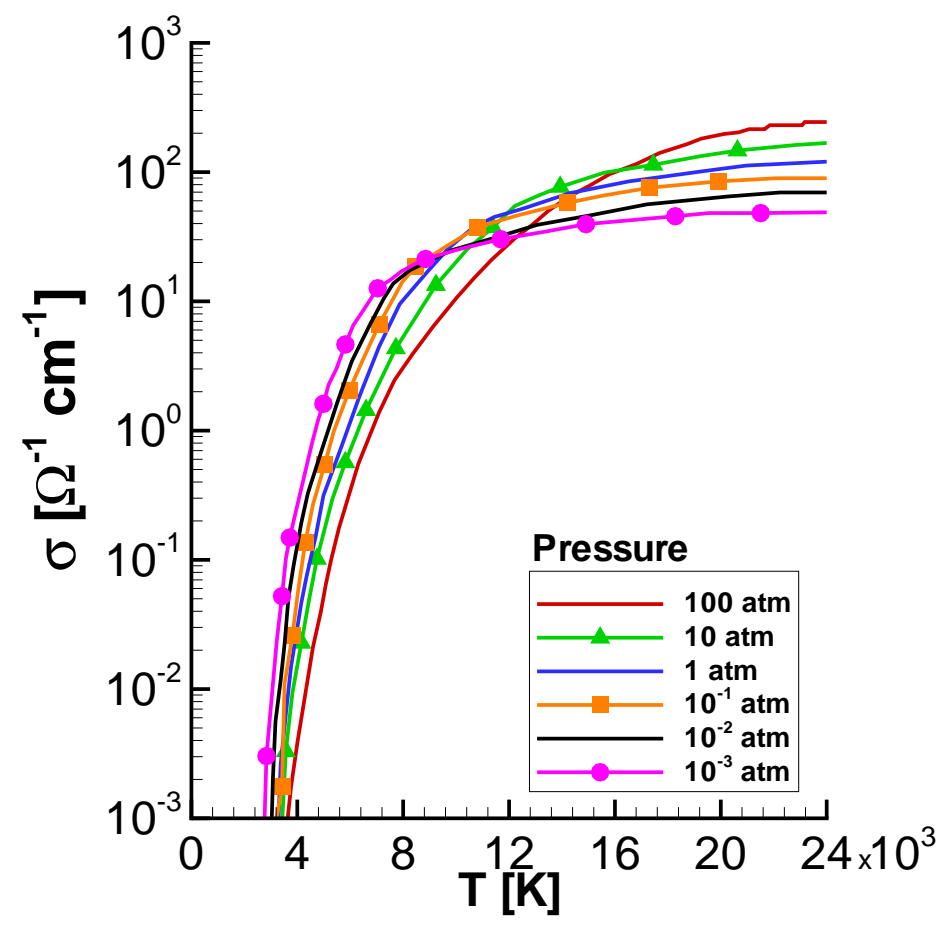

Figure 5.2: Electrical conductivity of equilibrium air for various temperatures and pressures, reproduced from Sutton and Sherman [93].

\subsubsection{Semi-Analytic Models}

Two of the first semi-analytic models developed from simplified versions of Boltzmann equations are the Spitzer-Härm model and the Chapman-Cowling model as outlined in [106]. The Spitzer-Härm model is derived to express the electrical conductivity of fully ionized gases by relating it to that of a Lorentz gas. A Lorentz gas is a model of a completely ionized gas in which electron-electron interactions are ignored and positive ion interactions do not exist because the ions are assumed stationary [107]. Because of these assumptions, the Spitzer-Härm model is only a function of temperature $\mathrm{T}[\mathrm{K}]$, and electron number density $n_{\mathrm{e}}\left[\mathrm{cm}^{-3}\right]$, as seen in Eqn. 5.5: 


$$
\sigma=\frac{1.56 \times 10^{-4} \times \mathrm{T}^{3 / 2}}{\ln \left(1.23 \times 10^{4} \times \mathrm{T}^{3 / 2} / \sqrt{n_{\mathrm{e}}}\right)} \Omega^{-1} \mathrm{~cm}^{-1}
$$

At the opposite extreme, Chapman and Cowling developed a model for a weaklyionized gas by assuming there is a coupling between the charge and mass diffusion terms and that the resultant electron energy distribution function from solutions to Boltzmann's equation is only a function of this coupled, binary diffusion coefficient. This assumption results in a semi-analytic model for the electrical conductivity, as seen in Eqn. 5.6:

$$
\sigma=3.34 \times 10^{-12} \frac{\alpha}{Q \sqrt{\mathrm{T}}} \Omega^{-1} \mathrm{~cm}^{-1}
$$

where $Q\left[\mathrm{~cm}^{2}\right]$ is the collision cross section of the gas, and the degree of ionization $\alpha=\Sigma n_{\text {ions }} / N$. One limitation of using the Chapman and Cowling model is that $Q$ must be determined by an outside source (i.e., experimental data, reference tables, an ideal molecule approximation, etc.). For the remainder of the chapter, the collision cross-section is taken to be the total collision cross-section for argon-argon collisions using hard sphere model[108], with a diameter of $4.04 \times 10^{-10} \mathrm{~m}[109]$, to yield: $Q \simeq 5 \times 10^{-17} \mathrm{~cm}^{2}$, unless otherwise stated. This assumption is made because it is unclear what the best choice for $Q$ should be, and because this assumption produces results that are consistent with the other semi-analytic models across the range of interest. Note that neither the Spitzer-Härm model nor the Chapman-Cowling model depend on the gas pressure or the species composition.

Instead of solving a reduced form of the Boltzmann equation, Bush developed an electrical conductivity model for investigating the boundary layer equations when a normal magnetic field is applied [11]. In order to achieve a similarity solution, the electrical conductivity was required to be a function of enthalpy. This results in an 
electrical conductivity model that is only a function of temperature [110], as seen in Eqn. 5.7:

$$
\sigma=\sigma_{0}\left(\frac{\mathrm{T}}{\mathrm{T}_{0}}\right)^{n}
$$

where $\sigma_{0}$ and $\mathrm{T}_{0}$ are reference electrical conductivity and temperature values, respectively. The reference conditions are determined from the peak temperature downstream of the bow shock for a hypersonic flow. Bush employed an exponent of $n=4$, although Otsu et al. have adopted and modified this approach by setting $n=2$ [111, 112]. Table 5.1 lists some typical reference values for weakly-ionized air based on experiments in [113] for standard atmospheric pressure. For the remainder of the chapter, $\sigma_{0}=51 \Omega^{-1} \mathrm{~cm}^{-1}$ at $\mathrm{T}_{0}=12000 \mathrm{~K}(p=1 \mathrm{~atm})$ is employed, unless otherwise stated.

Table 5.1: Experimental measurements of electrical conductivity in air at $p=1$ atm from [113]. ( \pm 20 percent experimental uncertainty)

\begin{tabular}{|c|c|}
\hline $\mathbf{T}[\mathbf{K}]$ & $\sigma\left[\Omega^{-1} \mathbf{c m}^{-1}\right]$ \\
\hline 6000 & 0.8 \\
\hline 7000 & 3.3 \\
\hline 8000 & 7.6 \\
\hline 9000 & 17 \\
\hline 10000 & 32 \\
\hline 12000 & 51 \\
\hline
\end{tabular}

Raizer developed an electrical conductivity model that is an exponential function of temperature, assuming that electron-neutral collisions affect the conductivity more than the electron-ion collisions and that the ionization is in thermal equilibrium [114], as seen in Eqn. 5.8: 


$$
\sigma=83 \times e^{-36000 / \mathrm{T}} \Omega^{-1} \mathrm{~cm}^{-1}
$$

where the temperature $\mathrm{T}$ is specified in Kelvin. This model is considered valid for air, nitrogen, and argon at $\mathrm{p}=1 \mathrm{~atm}$ for a temperature range of 8000 to $14000 \mathrm{~K}$. However, the model's coefficients (83 and -36000), can be adjusted depending on the temperature range, pressure, or gas composition of interest, but are used here as specified by Raizer, unless otherwise stated.

\subsubsection{Comparisons}

To facilitate comparisons between the existing electrical conductivity models, the species mole fractions for air at thermal equilibrium are generated for a range of pressures and temperatures by employing a computational equilibrium composition code developed by Godin and Trépanier [115]. The composition profiles are plotted in Fig. 5.3 for $p=10^{-3}$ atm and 1 atm. These profiles are used as input parameters to the Boltzmann solver and the other semi-analytic models being evaluated, and are tabulated at specific temperatures in Appendix D. As seen in the figures, the composition becomes fully ionized $(\alpha>>1 \%)$ for $\mathrm{T} \gtrsim 10^{4} \mathrm{~K}$.

Figure 5.4 shows the electrical conductivity distributions of all the models versus temperature at $p=10^{-3}$ atm and $1 \mathrm{~atm}$. The three lines representing solutions from the Boltzmann solver are computed for $\mathrm{E} / N=0.01,1$, and 100 Townsend $(\mathrm{Td})$,

respectively. ( $\left.1 \mathrm{Td}=10^{-17} \mathrm{~V} \cdot \mathrm{cm}^{2}\right)$ None of the semi-empirical models fully captures the behavior of the Boltzmann solver, although the Chapman-Cowling model appears to be the closest over the entire range, which is probably due to its dependence on the degree of ionization, as seen in Eqn. 5.6. Since Fig. 5.4 is plotted on a semi$\log$ scale, we see that the electrical conductivity dependence on $\mathrm{E} / N$ increases with 


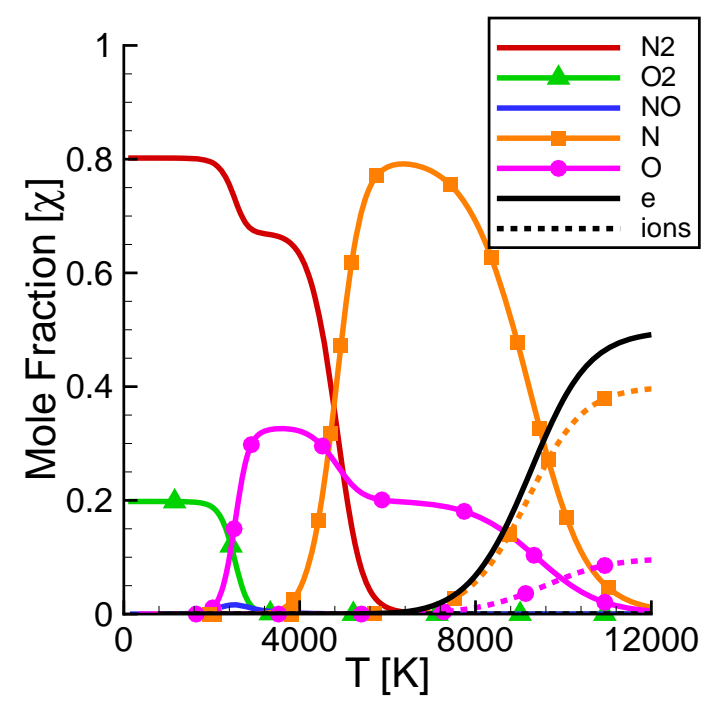

(a) $p=0.001 \mathrm{~atm}$

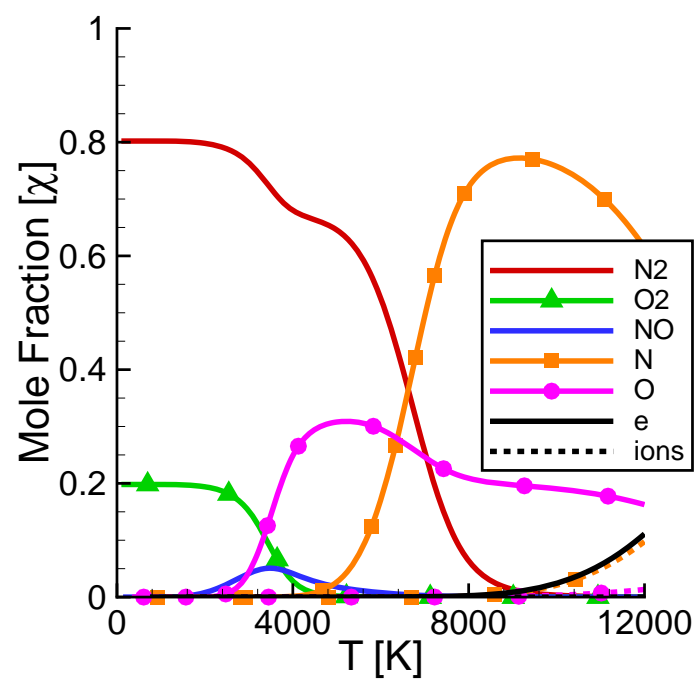

(b) $p=1.0 \mathrm{~atm}$

Figure 5.3: Mole fractions for 11 species equilibrium air versus temperature for two pressures.

temperature (or $\alpha)$.

In order to verify that the solutions produced by the Boltzmann solver are reasonable, experimental measurements collected by Lamb and Lin are compared against solutions from the Boltzmann solver for the range of temperatures observed in the experiment [116]. In the experiment, an axisymmetric magnetic field is aligned with a shock tube. The gas within the tube is initially at rest, but is then compressed to high temperature and starts to move due to the shock wave. The shock-heated gas travels into the magnetic field region and displaces the magnetic field, which is measured. Since the shock is electrically conductive due to thermal ionization, the displacement of the magnetic field lines is directly related to the electrical conductivity of the gas, which is measured versus the shock speed. The shock is assumed to achieve thermal equilibrium, so the corresponding temperature and species composition are estimated using the Rankine-Hugoniot equations across the shock front and existing tables for equilibrium air composition. Figure 5.5 plots the measured 


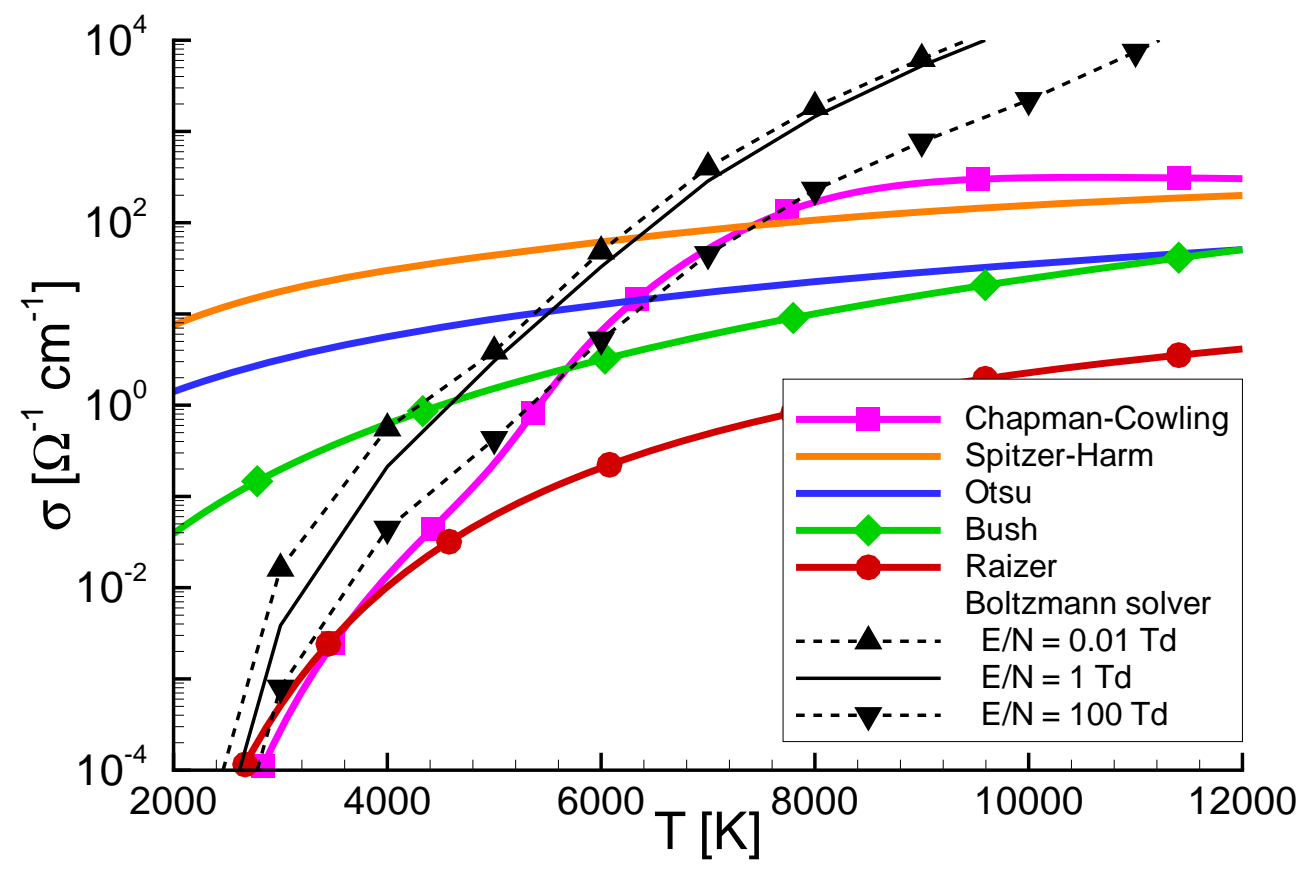

(a) $p=0.001 \mathrm{~atm}$

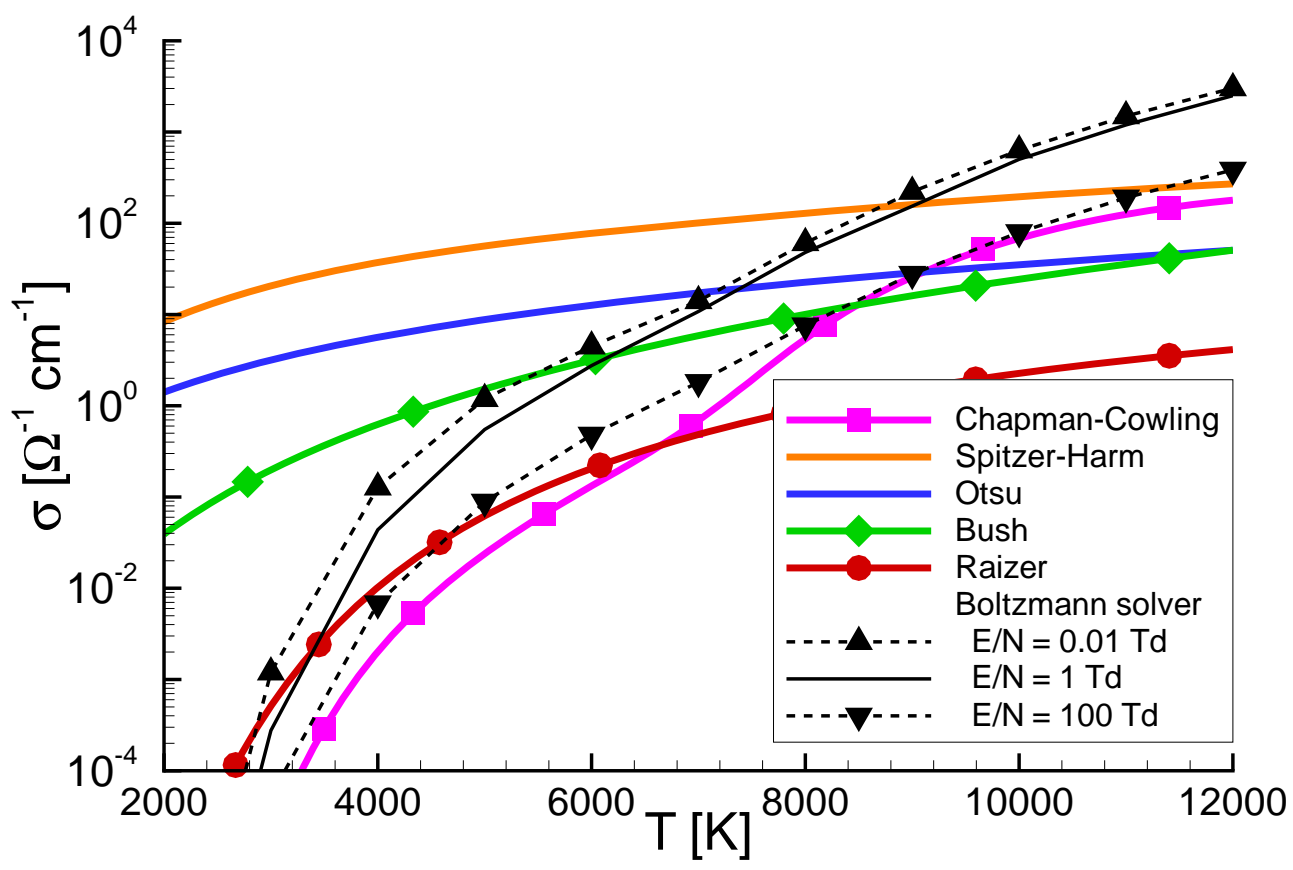

(b) $p=1.000 \mathrm{~atm}$

Figure 5.4: Electrical conductivity versus temperature from various models for two pressures. 
electrical conductivity and estimated temperature for various shock Mach numbers.

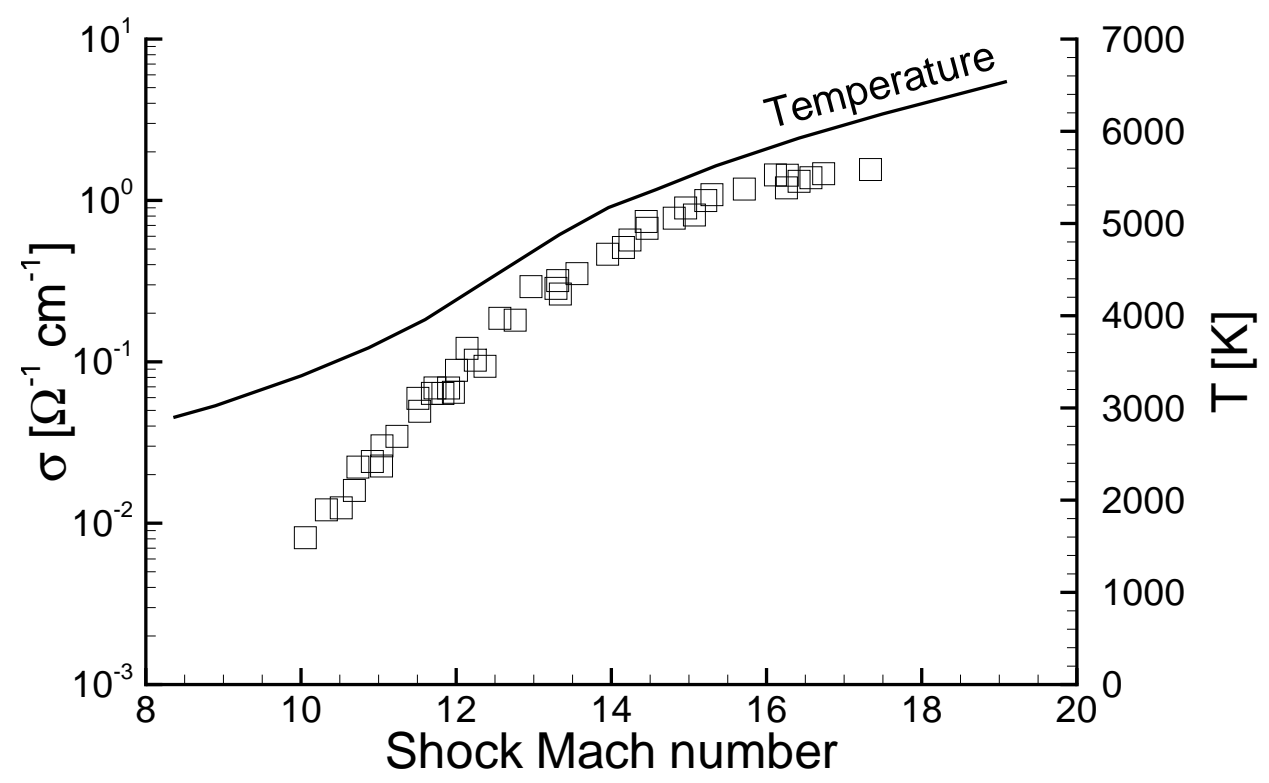

Figure 5.5: Electrical conductivity and temperature of equilibrium air $(p=0.001$ atm), for various shock Mach numbers. Reproduced from Lamb and Lin [116]. ( \pm 20 percent experimental uncertainty)

Relating the temperature to the electrical conductivity, Fig. 5.6 plots the measured electrical conductivity versus temperature. In addition, the plot also includes solutions from the Boltzmann solver for $p=0.001$ atm using the species compositions listed in Table D.1 (located in Appendix D), for specified temperatures in the range of those obtained in the experiment. The solutions from the Boltzmann solver appear to match the experiments within the uncertainty of the measurements.

Table 5.2 lists the computational time in seconds necessary to compute the electrical conductivity for ten thousand cells for each of the methods described. The time estimates are based on the average results from the simulations computed to populate Fig. 5.4. Although the Boltzmann solver provides accurate solutions for the entire temperature range, it is computationally prohibitive to use directly for each cell of the combined fluid-MHD code. 


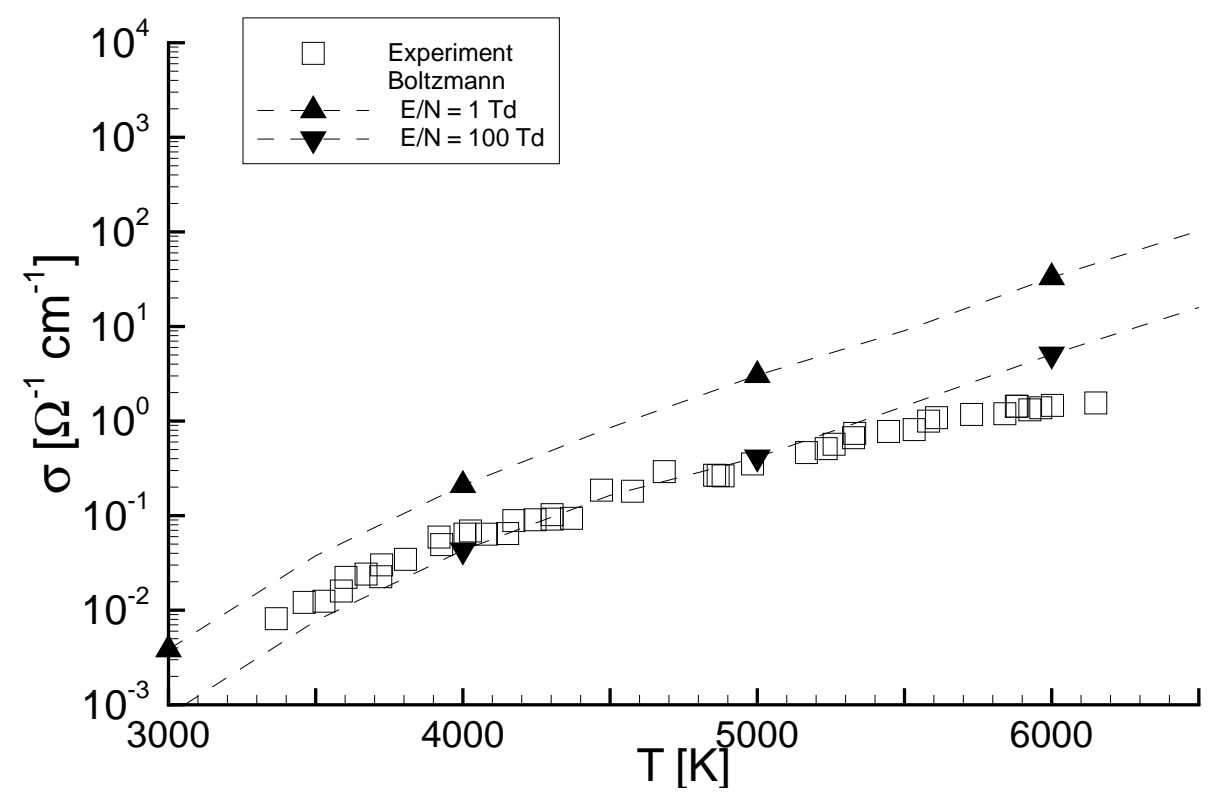

Figure 5.6: Electrical conductivity versus temperature for equilibrium air $(p=0.001$ atm). Experimental measurements from [116]. (symbol size reflects \pm 20 percent experimental uncertainty)

Table 5.2: Computational cost (CPU-seconds) to determine the electrical conductivity for ten thousand finite-volume cells .

\begin{tabular}{cc|cccc}
\hline \hline \multicolumn{2}{c|}{ Boltzmann solver } & Spitzer & Chapman & & \\
w/ e-e & w/o e-e & -Härm & -Cowling & Bush & Raizer \\
\hline $5.4 \times 10^{6}$ & $1.7 \times 10^{3}$ & $4.5 \times 10^{-3}$ & $13 \times 10^{-3}$ & $4.3 \times 10^{-3}$ & $4.7 \times 10^{-3}$ \\
\hline \hline
\end{tabular}




\subsection{Surrogate Modeling}

The lack of generality and accuracy amongst the semi-analytic models, along with the high computational cost of a direct Boltzmann solution, motivates the development of an alternative conductivity model. Surrogate modeling, an approach used in many scientific fields $[117,118,119,120]$, is selected to develop the new electrical conductivity model because it provides generality, and has several welldocumented approaches immediately available. The basic idea of surrogate modeling is to develop (or teach) a model by supplying a number of sample points which encompasses the domain of interest (design space), and then evaluate the model's accuracy using additional test points. This work uses a surrogate modeling tool suite, SURROGATES Toolbox, developed by Goel and Viana [121], which integrates

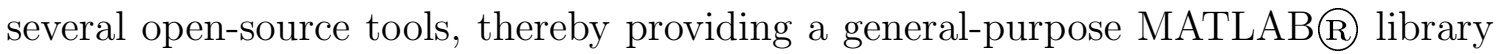
of multidimensional function approximation methods.

\subsubsection{Surrogates}

While there are a number of surrogate models available in the literature to correlate solutions to Boltzmann's equations, SURROGATES Toolbox currently features four of the most widely used approximations. The Polynomial Response Surface (PRS), Kriging (KRG), Radial Based Neural Network (RBNN), and Support Vector Regression (SVR) models each have advantages and disadvantages depending on the data set being approximated. For example, the Kriging method estimates the response (solution) based on a known function (e.g., a linear polynomial), and its corresponding deviation for the sample point. It is typically employed when the data appear noisy or erratic, and because of its definition, results in a high correlation between the model and the actual data provided. However, it is not usually em- 
ployed because of its complexity and high computational expense [122]. The PRS model is a commonly employed method, which uses a polynomial function of degree $n$ to fit the data. The simplicity of PRS makes it attractive and easy to implement, but it may require a high-order polynomial to capture highly non-linear data sets. A thorough description of each of the models (PRS, KRG, RBNN, and SVR), is available in [123], while [124] provides several example applications for the various models. This work utilizes the PRS model, though any of the other models could easily be substituted in the procedure to examine their suitability.

The $1^{\text {st }}$ Order PRS model is listed in Eqn. 5.9:

$$
\hat{\sigma}=c_{0}+\sum_{i=1}^{N D}\left(c_{i} d_{i}\right)
$$

where $\hat{\sigma}=$ is the predicted response, $c_{0}, \ldots, c_{i}$ are constant coefficients for the polynomial, $d_{i}$ is a dimension of the model or design space, and $N D$ is the total number of dimensions in the model. For example, in three-species argon ( $\mathrm{Ar}, \mathrm{Ar}^{+}$, and e), only the normalized electric field $\mathrm{E} / N$ and the first two species mole fractions are needed by the Boltzmann solver because of charge neutrality. So, the model dimensions are: $d_{1}=\mathrm{E} / N, d_{2}=\chi_{\mathrm{Ar}}$, and $d_{3}=\chi_{\mathrm{Ar}^{+}}$.

For the $1^{\text {st }}$ order model, only four constant coefficients exist, while the $2^{\text {nd }}$ order has ten constant coefficients, as seen in Eqn. 5.10:

$$
\hat{\sigma}=c_{0}+\sum_{i=1}^{N D}\left(c_{i} d_{i}\right)+\sum_{i=1}^{N D} \sum_{j=i}^{N D}\left(c_{i, j} d_{i} d_{j}\right)
$$

where $c_{0}, \ldots, c_{i, j}$ are constant coefficients for the polynomial and go from $c_{0}$ to $c_{3,3}$. Likewise, the method can be expanded for any order of dimensions by adding an additional summation series, as seen in Eqn. 5.11 for a $3^{\text {rd }}$ Order PRS model: 


$$
\hat{\sigma}=c_{0}+\sum_{i=1}^{N D}\left(c_{i} d_{i}\right)+\sum_{i=1}^{N D} \sum_{j=i}^{N D}\left(c_{i, j} d_{i} d_{j}\right)+\sum_{i=1}^{N D} \sum_{j=i}^{N D} \sum_{k=j}^{N D}\left(c_{i, j, k} d_{i} d_{j} d_{k}\right)
$$

which has $c_{0}, \ldots, c_{i, j, k}, c_{3,3,3}(20)$ coefficients. For any polynomial, the maximum number of constant coefficients is determined using Eqn. 5.12:

$$
\frac{(k+n) !}{(k ! n !)}=\text { total number of coefficients }
$$

where $k$ is the number of dimensions and $n$ is the order of the polynomial. Table 5.3 lists the total number of coefficients for several dimensions and the order of the polynomial (degree of the polynomial). As seen in the table, the number of constant coefficients in a polynomial increases dramatically with the dimensions or the degree of the polynomial. The number of constant coefficients is related to the computational cost necessary to utilize a particular PRS model, which factors into the selection of the most suitable model.

Table 5.3: Total number of constant coefficients for an $n^{\text {th }}$ order polynomial in $k$ dimensions (Eqn. 5.12).

\begin{tabular}{cc|ccccccccccc}
\hline \hline & & \multicolumn{1}{c}{} & \multicolumn{10}{c}{ Number of Dimensions } \\
& & $\mathbf{1}$ & $\mathbf{2}$ & $\mathbf{3}$ & $\mathbf{4}$ & $\mathbf{5}$ & $\mathbf{6}$ & $\mathbf{7}$ & $\mathbf{8}$ & $\mathbf{9}$ & $\mathbf{1 0}$ & $\mathbf{1 1}$ \\
\hline \multirow{3}{*}{ Order } & $\mathbf{1}$ & 2 & 3 & 4 & 5 & 6 & 7 & 8 & 9 & 10 & 11 & 12 \\
& $\mathbf{2}$ & 3 & 6 & 10 & 15 & 21 & 28 & 36 & 45 & 55 & 66 & 78 \\
& $\mathbf{3}$ & 4 & 10 & 20 & 35 & 56 & 84 & 120 & 165 & 220 & 286 & 364 \\
& $\mathbf{4}$ & 5 & 15 & 35 & 70 & 126 & 210 & 330 & 495 & 715 & 1001 & 1365 \\
& $\mathbf{5}$ & 6 & 21 & 56 & 126 & 252 & 462 & 792 & 1287 & 2002 & 3003 & 4368 \\
& $\mathbf{6}$ & 7 & 28 & 84 & 210 & 462 & 924 & 1716 & 3003 & 5005 & 8008 & 12376 \\
\hline \hline
\end{tabular}




\subsubsection{Dimensions}

As previously mentioned, the Boltzmann solver depends on the normalized electric field $\mathrm{E} / N$, and each species mole fraction $\chi_{s}$. These input parameters form the dimensions of the surrogate model. While this thesis primarily computes flow-fields in air (employing either five or eleven species), the solver must also be suitable for other gas compositions, such as weakly-ionized argon. Because argon is easily ionized, easily obtained, and a noble gas (which removes complex gas chemistry), it is an ideal composition for many hypersonic experiments which focus on other flow phenomena $[16,30,125]$.

Weakly-ionized argon (Ar, $\mathrm{Ar}^{+}$, and e) is used in this section to explain the procedure of developing a surrogate model because it can be modeled with three species mole fractions as compared to eleven species in air, thereby significantly reducing the complexity and dimensions of the Design of Experiment (DOE). (Weakly-ionized argon could also include $\mathrm{Ar}^{*}, \mathrm{Ar}_{3}^{+}$, etc., but these are ignored.) Nonetheless, the procedure outlined in this section is independent of the gas composition selected. In addition, the electrical conductivity profile for weakly-ionized argon is similar to air, as seen in Fig. 5.7, so the resulting models should have similar characteristics.

Because of charge neutrality, the DOE only has three dimensions: $\mathrm{E} / N, \chi_{\mathrm{Ar}}$, and $\chi_{\mathrm{Ar}^{+}}$. The limits on the DOE are set so the normalized electric field varies from 0.01 to $100 \mathrm{Td}$, any neutral species mole fraction ranges from $0 \%$ to $100 \%$, and any ionic species from $0 \%$ to $0.1 \%$. A $0.1 \%$ maximum mole fraction for the ionic species is imposed because the MHD solver developed in Section 4.2.1 is only intended for weakly-ionized flows [106] $(\alpha \ll 1 \%)$.

A random set of sample points is produced using the Huge Latin Hyper Cube (HLHC) tool in the SURROGATES Toolbox. HLHC populates the DOE randomly 


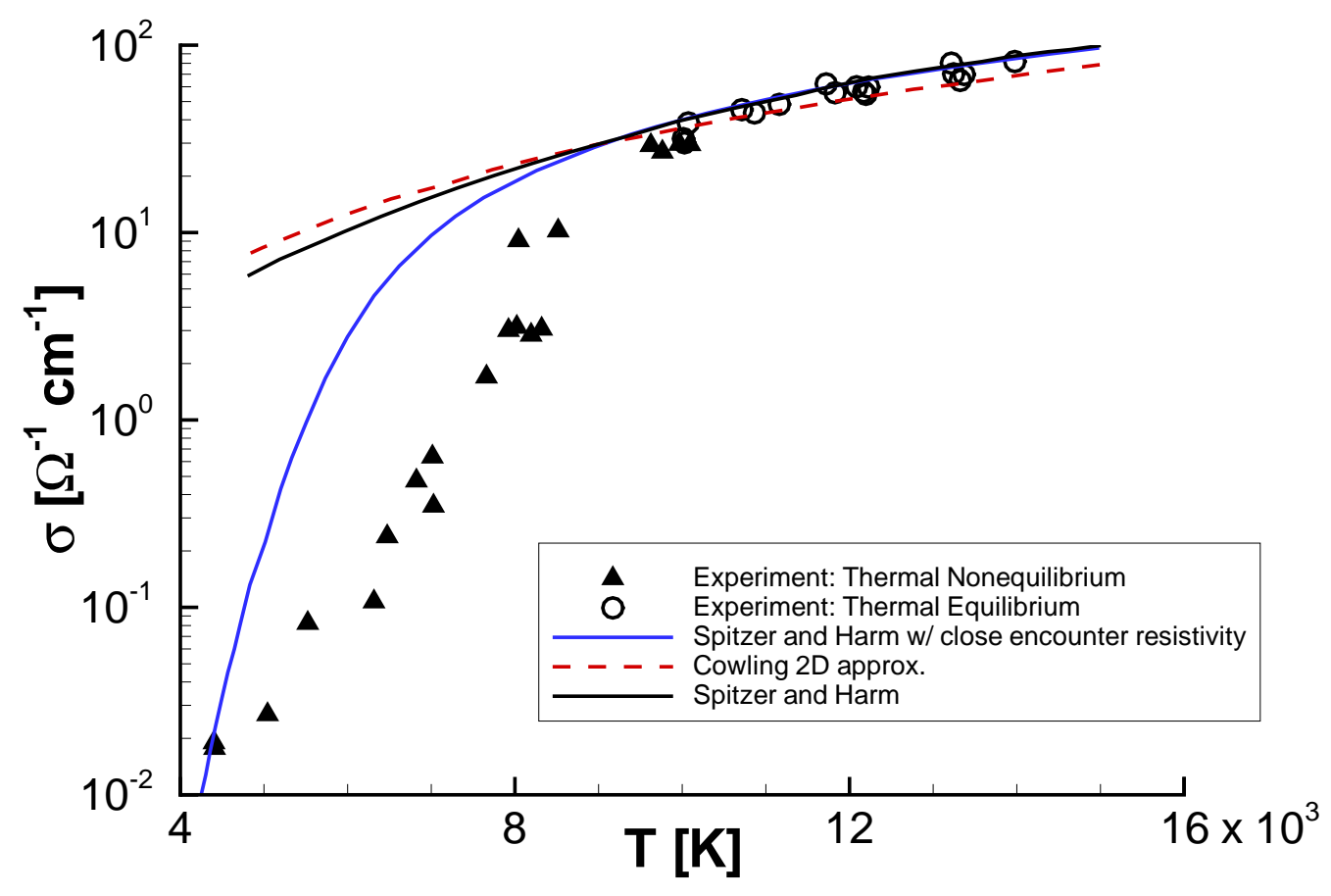

Figure 5.7: Electrical conductivity of argon $(p=0.013 \mathrm{~atm})$, reproduced from Lin et al. [126].

across all mole fraction dimensions while enforcing a conditional statement which maximizes the minimum distance between dimensions of the sample points in order to reduce the correlation of the sample points generated [121]. A generated sample point is only kept if the summation of all species mole fractions equals one and the summation of the ionic species mole fractions is less than or equal to $0.1 \%$.

The HLHC tool is not used to generate points in the normalized electric field dimension $\mathrm{E} / N$, because the Boltzmann solver computes solutions for a gas composition at specified values of $\mathrm{E} / N$. Although these values of $\mathrm{E} / N$ are adjustable, the solutions obtained in Fig. 5.4 suggest that the electrical conductivity solutions should be adequately sampled by using several consistent, evenly spaced values (e.g., $\mathrm{E} / N=0.01 \mathrm{Td}, 0.03 \mathrm{Td}, 0.05 \mathrm{Td}$, etc. for all gas composition combinations).

A typical number of 'learning' points found in surrogate modeling literature is 
between $(1.5-2.0) \times 2^{n}$ points, where $n$ is the number of dimensions. This guideline stems from the idea that each sub-domain of the DOE should have at least one to two points (e.g., a two-dimensional DOE has 4 sub-domains, a three-dimensional DOE has 8, etc.). Additional points improve the accuracy of the response (solution model), but additional sample points can be computationally wasteful so care should be taken when determining the number of points necessary to capture the behavior of the response.

Since weakly-ionized argon only has one neutral $\chi_{\mathrm{Ar}}$, and one ionic species $\chi_{\mathrm{Ar}^{+}}$, the value of the ionic species mole fraction is known once the neutral fraction has been determined $\left(\chi_{\mathrm{Ar}^{+}}=1-\chi_{\mathrm{Ar}}\right)$. Since the number of minimum points necessary to populate the DOE is only 20, additional points are also computed to provide an improved model. Two hundred and twenty learning points (27.5 points per subdomain) and 180 testing points (22.5 points per sub-domain) are used to populate the DOE. The learning points are composed of eleven different $\mathrm{E} / N$ values, whereas the testing points use nine different $\mathrm{E} / N$ values, as illustrated in Fig. 5.8. Only two of the dimensions are plotted in the figure because a three-dimensional plot would result in a solution along the plane where $\chi_{\mathrm{Ar}^{+}}=1-\chi_{\mathrm{Ar}}$.

\subsubsection{Reduced Dimensional Modeling}

It is useful to minimize the dimensions of a surrogate model, especially since the number of sample points recommended to 'teach' the model escalates rapidly with the number of dimensions. In addition to the extra computational resources needed to compute the points, the number of variables in a PRS model directly relates to the model dimensions, making a large dimensional model less desirable to employ.

Solutions to Boltzmann's equation require the DOE to be constrained such that 


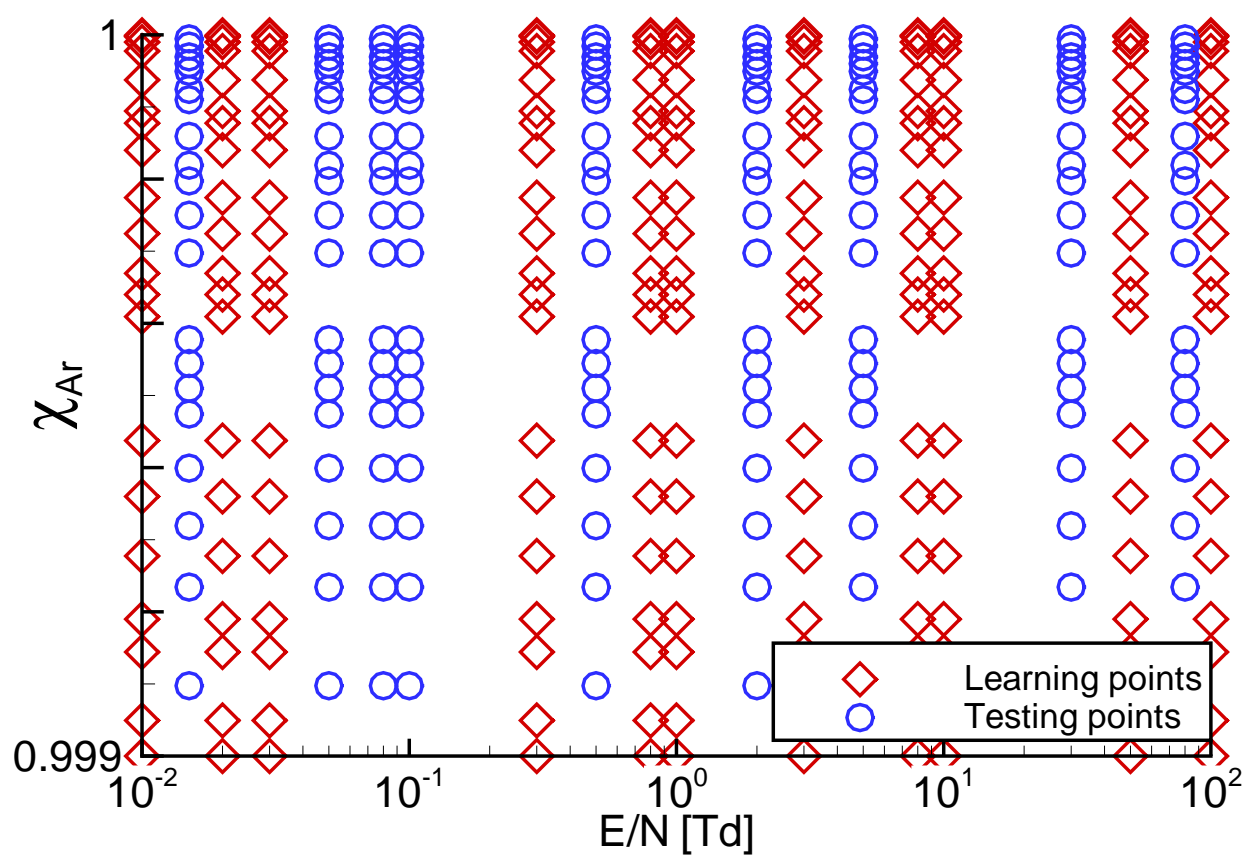

Figure 5.8: Design of Experiment 'learning' and 'testing' points for the threedimensional surrogate model of weakly-ionized argon.

the summation of all species mole fractions equals one and the summation of the ionic species mole fractions is less than or equal to $0.1 \%$. This is enforced by transforming the species mole fractions to spherical coordinates (with the radius set to one). This approach reduces the dimensions of the surrogate model by one without removing any input parameters to the Boltzmann solver. For example, suppose the surrogate model dimensions are for a mixture of neon and weakly-ionized argon (Ne, Ar, and $\mathrm{Ar}^{+}$), then the species could be represented in spherical coordinates, as seen in Eqn. 5.13:

$$
\begin{aligned}
\chi_{\mathrm{Ne}} & =\sin ^{2}(\lambda) \sin ^{2}(\theta) \\
\chi_{\mathrm{Ar}} & =\sin ^{2}(\lambda) \cos ^{2}(\theta) \\
\chi_{\mathrm{Ar}^{+}} & =\cos ^{2}(\lambda)
\end{aligned}
$$


where $\lambda$ and $\theta$ are the inclination and azimuth angles representing positions along the surface of the sphere. Figure 5.9(a) illustrates the concept, which can be expanded to accommodate any number of species, where each angle varies from zero to $\pi / 2$. However, the example selected includes an ionized species $\left(\mathrm{Ar}^{+}\right)$, which is limited to a mole fraction less than or equal to $10^{-3}$. Therefore, the inclination angle $\lambda$, which is directly related to $\chi_{\mathrm{Ar}^{+}}$in Eqn. 5.13, is constrained using Eqn. 5.16:

$$
\lambda_{\min }=\cos ^{-1}\left(\sqrt{\chi_{\max }}\right)
$$

where $\chi_{\max }$ is the maximum mole fraction allow ( $0.1 \%$ for ions, $100 \%$ for neutrals), and $\lambda_{\min }$ is the resulting minimum angle that can be selected to ensure the resulting mole fraction is logical. As a result, for the ionic species angle $\lambda_{\min }=1.539$ radians, so: $1.539 \leq \lambda \leq \pi / 2$. Since each neutral species can vary from 0 to $100 \%$, the azimuth angle is not restricted: $0 \leq \theta \leq \pi / 2$. These restrictions can be seen visually in Fig. 5.9(b) where the shaded part of the shell indicates the correct combination of angles such that all resulting species mole fractions are valid.

The main consequence of using the species angles as dimensions of the DOE is that the definition of each angle does not directly correspond to a specific species mole fraction. In fact, for a gas composition with many species, each angle represents a large combination of trigonometric functions and species mole fractions, as seen in Appendix E for 11 species air. Using mole fraction angles in the DOE can be potentially problematic, especially when identifying which species are important to the response since the method increases the correlation each dimension has on the response and reduces the likelihood of removing dimensions based on global sensitivity analysis. In addition, the complex representation of each species mole fraction can be computationally expensive to implement into the resultant electrical conductivity 


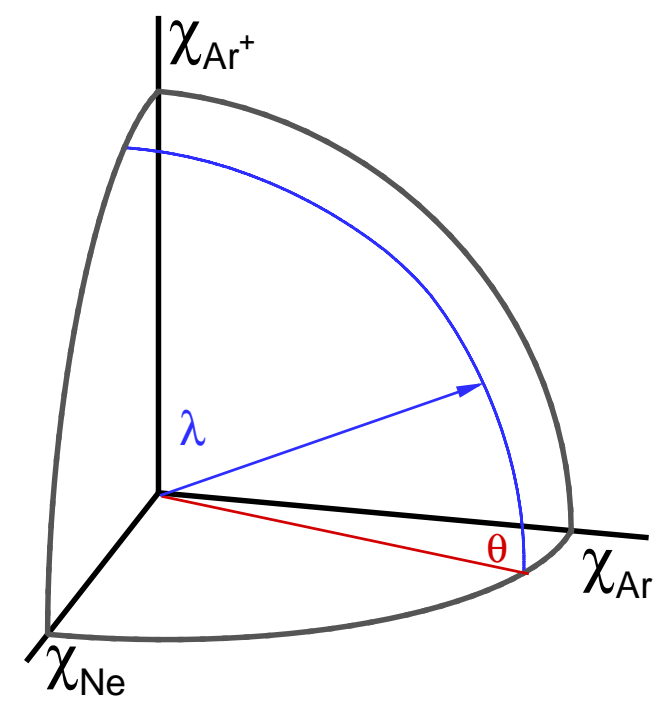

(a) Cartoon showing the spherical coordinate transformation.

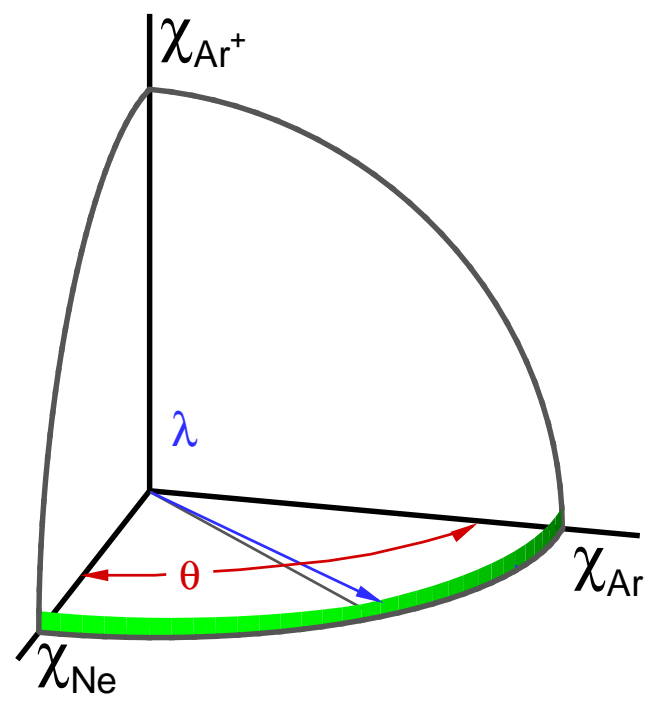

(b) Surface (green) indicates region valid combinations of angles.

Figure 5.9: A spherical coordinate transformation representing a three species gas composition. The radius of the eighth of the sphere equals one.

model. As such, the weakly-ionized argon model being developed in this section will not use the mole fraction angles as its species dimensions, though the transformation is used by the HLHC tool to populate the DOE.

\section{Model Formulation}

With the DOE determined, solutions to the points are obtained using grid computing with individual Boltzmann solutions run in parallel, allowing for electronelectron collisions. Figure 5.10 plots the resulting electrical conductivity contours, which show a region of high conductivity for low $\chi_{\text {Ar }}$ (high degree of ionization), and a weak normalized electric field $\mathrm{E} / N$.

Decreasing electrical conductivity for an increasing electric field strength is also seen in Fig. 5.4, where the Boltzmann solver results are shown for $\mathrm{E} / N=0.1,1,100$ Td. As such, the electrical conductivity appears to be a function of $\mathrm{E} / N$, which is anticipated by factoring the electron number density out of the electrical conductivity 


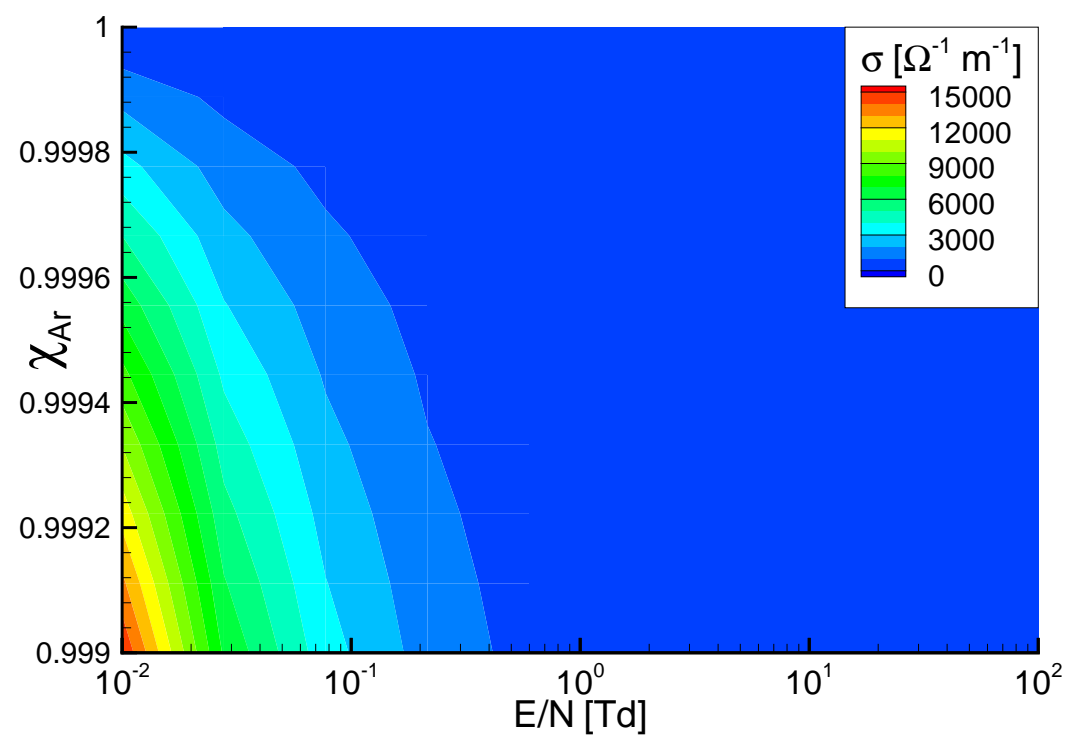

Figure 5.10: Electrical conductivity contours for weakly-ionized argon.

in Eqn. 5.1 to yield: $\sigma / n_{\mathrm{e}} \sim \nu_{m}^{-1}$. Since the electron collision frequency, $\nu_{m}$, usually increases with increasing $\mathrm{E} / N$, as seen in Fig. 5.11, the electrical conductivity should decrease with increasing electric field strength.

Since the model's dependent variable, $\sigma$, has a large range of values, surrogate models may have trouble capturing the behavior, especially in regions with a large gradient. To help SURROGATES Toolbox create a highly accurate model, it is useful to transform the function that the PRS model is trying to mimic, by reducing the dependent variable's range. Dividing the electrical conductivity by the degree of ionization does not require any additional information (parameters), since $\alpha=\Sigma \chi_{\text {ions }}=\chi_{\mathrm{Ar}^{+}}$, but helps to normalize the solution. However, this leads to a division by zero error when $\alpha=0$, so the dependent variable is inverted: $\alpha / \sigma$. This transformation is similar to the Chapman-Cowling model, which also utilizes the degree of ionization in the numerator. The dependent variable is small for this formulation $\left(10^{-8} \Omega \cdot \mathrm{m} \leq \alpha / \sigma \leq 10^{-6} \Omega \cdot \mathrm{m}\right)$, so the natural logarithm is also applied to renormalize the solution range. Equation 5.17 lists the model formulation provided 


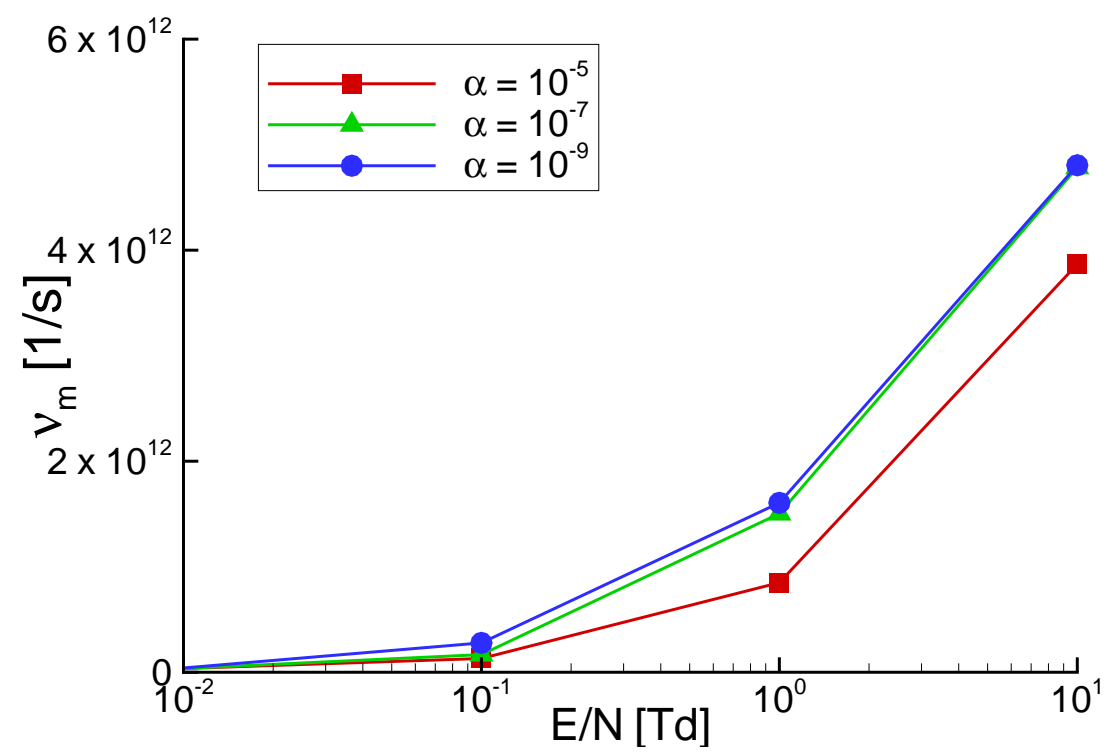

Figure 5.11: Electron collision frequency for weakly-ionized argon at $p=1 \mathrm{~atm}$.

to SURROGATES Toolbox:

$$
\ln \left(\frac{\alpha}{\sigma}\right)=f\left(\mathrm{E} / N, \chi_{\mathrm{Ar}}, \chi_{\mathrm{Ar}^{+}}\right)
$$

Applying the natural logarithm function provides a second advantage when the model is employed. Since the formulated model is a function of the natural logarithm and the degree of ionization, the electrical conductivity must be extracted from the model solution by dividing the degree of ionization by the exponential function of the model's prediction: $\sigma=\alpha / \exp \left(\operatorname{PRS}\left(\mathrm{E} / N, \chi_{\mathrm{Ar}}, \chi_{\mathrm{Ar}^{+}}\right)\right)$. Since the exponential of any real number (the result of using the PRS model), is positive, the resulting electrical conductivity predicted will always be greater than or equal to zero. This characteristic (the model will always provide a positive value), is critical for the implementation of the model, since the electrical conductivity of a real gas is always greater than or equal to zero.

Although this formulation of the model incurs additional computational expense (i.e., evaluation of the exponential function), higher accuracy is achieved for lower 
order PRS models because the gradients within the surface are reduced. Figure 5.12 plots the resulting $1^{\text {st }}$ to $6^{\text {th }}$ order polynomial response surfaces. As seen in the figure, the $1^{\text {st }}$ order model appears to under-predict the response, while the $2^{\text {nd }}$ order model appears to over-predict it in the peak electrical conductivity region. The higher order PRS models $\left(3^{\text {rd }}-6^{\text {th }}\right)$, all appear to predict similar surfaces.

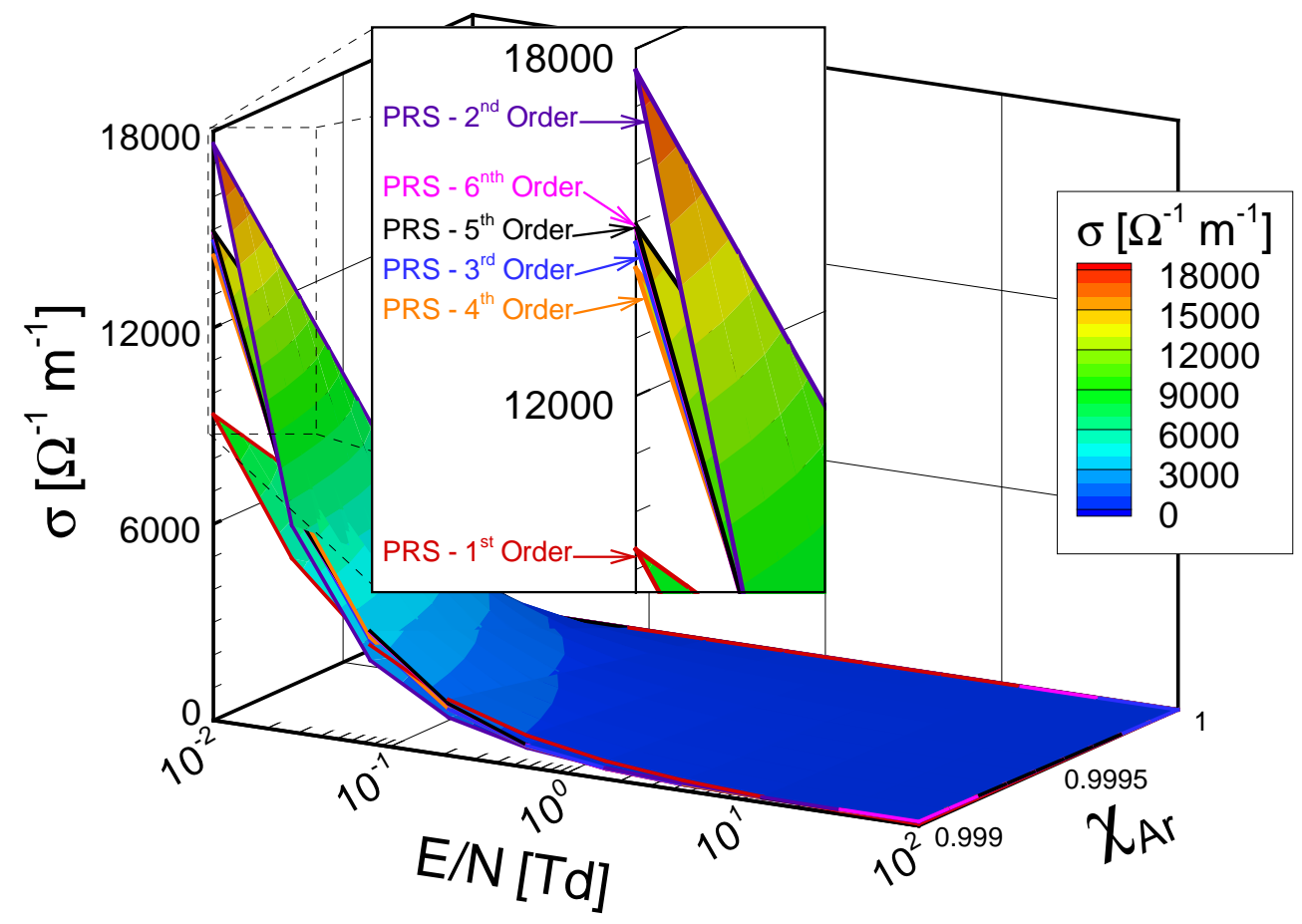

Figure 5.12: Various PRS model surfaces for weakly-ionized argon.

Additional information about the formulation of the model dimension variables and the constant coefficients of the PRS models developed is available in Appendix F.

\section{Approximate Error}

PRESS is a tool in the SURROGATES Toolbox which determines the Root Mean Square Error (RMSE) of the model by using the sample points already provided to teach the model [127]. Unfortunately, the cost of this tool is computationally 
prohibitive when the number of 'learning' points is large ( $\geq 100$ points). Instead, this chapter determines the accuracy of the PRS model using additional 'testing' points which are determined using the same method as the 'learning' points described previously.

A common way of quantifying the accuracy of the model is to calculate the error at each 'test' point (i.e., standard error), and assess the results using the RMSE. However, like all statistics, the RMSE is a disputable indicator of the model's accuracy because outliers ('test' points that perform poorly compared to others), have a larger influence on the metric [128]. Since the electrical conductivity can vary by several orders of magnitude depending on the pressure and temperature, as seen in Fig. 5.2 for equilibrium air, the statistic is biased toward smaller standard error at low conductivity because it does not account for the local actual value.

The Percent Error (PE) accommodates the large range of electrical conductivities by dividing the standard error by the local actual value, but can be misleading when the local actual value is less than one, since it will inflate the percent error. In addition, the standard PE is biased toward over-predictions, but both these issues are corrected by normalizing the standard error by the average of the predicted and actual values [129], and this method is used throughout this thesis.

Another challenge is that a surrogate model that exactly matches the 'test' points would have a $\mathrm{PE}=0 \%$, which provides the lower bound, but not an upper bound. To obtain an upper bound, one additional metric is developed which measures the Exponential of the negative absolute Percent Error (ePE), because the exponential of a negative value is bounded from one to zero for all real positive values. To be consistent with the other computed metrics (i.e., zero being an exact fit), the metric is reflected about the $y=0.5$ axis. 
Table 5.4 lists the metrics and their definitions, while Table 5.5 provides example scenarios to illustrate the similarities and differences between the various error definitions and metrics. Ultimately, it is important to consider all the metrics when quantifying the accuracy of a specific model because each provides specific insight into the model's performance.

Table 5.4: Definitions of error metrics ( $\hat{y}=$ predicted, $y=$ actual, $n$ points).

\begin{tabular}{|c|c|}
\hline Name & Definition \\
\hline Standard Error (E) & $\hat{y}-y$ \\
\hline Percent Error (PE) & $\frac{2(\hat{y}-y)}{\hat{y}+y}$ \\
\hline Exponential Percent Error (ePE) & $1-e^{-\left|\frac{2(\hat{y}-y)}{\hat{y}+y}\right|}$ \\
\hline - - - - - - - - - - - - - - - - & 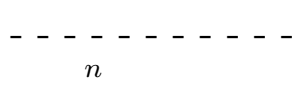 \\
\hline Mean Absolute Error (MAE) & $\frac{1}{n} \sum_{i=1}|\hat{y}-y|_{i}$ \\
\hline Root Mean Square Error (RMSE) & $\sqrt{\frac{1}{n} \sum_{i=1}^{n}(\hat{y}-y)_{i}^{2}}$ \\
\hline
\end{tabular}

Table 5.5: Example scenarios to demonstrate the error definitions.

\begin{tabular}{cc||ccc}
\hline \hline Actual $[y]$ & Predicted $[\hat{y}]$ & Error & PE [\%] & ePE \\
\hline 1000 & 1010 & 10 & 1.0 & 0.0099 \\
1000 & 700 & -300 & -35.3 & 0.2974 \\
0.5 & 0.7 & 0.2 & 33.3 & 0.2834 \\
0.7 & 0.5 & -0.2 & -33.3 & 0.2834 \\
0.1 & 1.5 & 1.4 & 175.0 & 0.8262 \\
\hline \hline
\end{tabular}

Several weakly-ionized argon electrical conductivity model are developed using the PRS approach for $1^{\text {st }}$ to $6^{\text {th }}$ order polynomial response surfaces. The resultant accuracy metrics are populated in Fig. 5.13(a) for the RMSE and in Fig. 5.13(b) 
for the Mean Absolute Error (MAE) using both the standard and the percent error. The figures indicate that the lower order polynomial models have high amounts of error, while the higher models level off without much improvement. Table 5.6 lists all the error metrics for the various models. While the third order model has the lowest standard error, the higher order models have slightly lower percent errors.

Table 5.6: Error metrics for weakly-ionized argon.

\begin{tabular}{c|cccccc}
\hline & \multicolumn{6}{|c}{ PRS model } \\
& $1^{\text {st }}$ & $2^{\text {nd }}$ & $3^{\text {rd }}$ & $4^{\text {th }}$ & $5^{\text {th }}$ & $6^{\text {th }}$ \\
\hline $\mathrm{MA}(\mathrm{E})\left[\Omega^{-1} \mathrm{~m}^{-1}\right]$ & 167.93 & 90.72 & 13.68 & 21.46 & 43.24 & 39.10 \\
$\mathrm{RMS}(\mathrm{E})\left[\Omega^{-1} \mathrm{~m}^{-1}\right]$ & 487.21 & 164.14 & 23.45 & 42.02 & 84.13 & 76.16 \\
$\mathrm{MA}(\mathrm{PE})[\%]$ & 34.26 & 16.35 & 6.85 & 7.13 & 5.82 & 5.49 \\
$\mathrm{RMS}(\mathrm{PE})[\%]$ & 42.25 & 18.32 & 10.36 & 9.97 & 7.27 & 6.85 \\
$\mathrm{MA}(\mathrm{ePV})$ & 0.268 & 0.148 & 0.063 & 0.067 & 0.056 & 0.053 \\
$\mathrm{RMS}(\mathrm{ePV})$ & 0.322 & 0.163 & 0.094 & 0.091 & 0.069 & 0.065 \\
\hline \hline
\end{tabular}

\subsubsection{Conservativeness}

Because the surrogate model developed represents a non-negative flow property (the electrical conductivity), and its predictions are used as an intermediate step within the flow solver, it is crucial that the model does not predict a negative response. This requirement, called conservativeness, can be estimated by determining what percent of the 'testing' points predict a positive response $(\hat{y} \geq 0)$. Note that the predicted response does not have to be greater than the actual value $(\hat{y}-y=\mathbb{R})$.

Because the model response is formulated by taking the natural logarithm of the degree of ionization divided by the electrical conductivity, as seen in Eqn. 5.17, the model's extracted electrical conductivity prediction will always be positive. This ensures the model's conservativeness is $100 \%$. However, if the response was formulated 


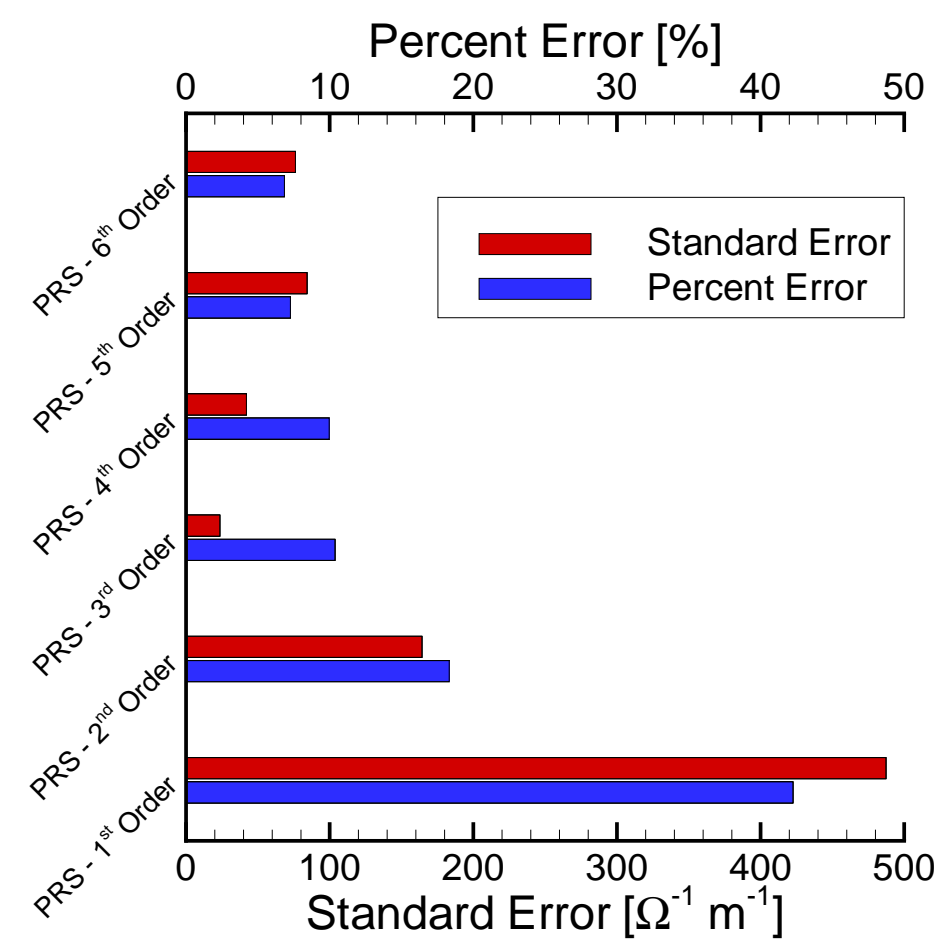

(a) Root mean square error.

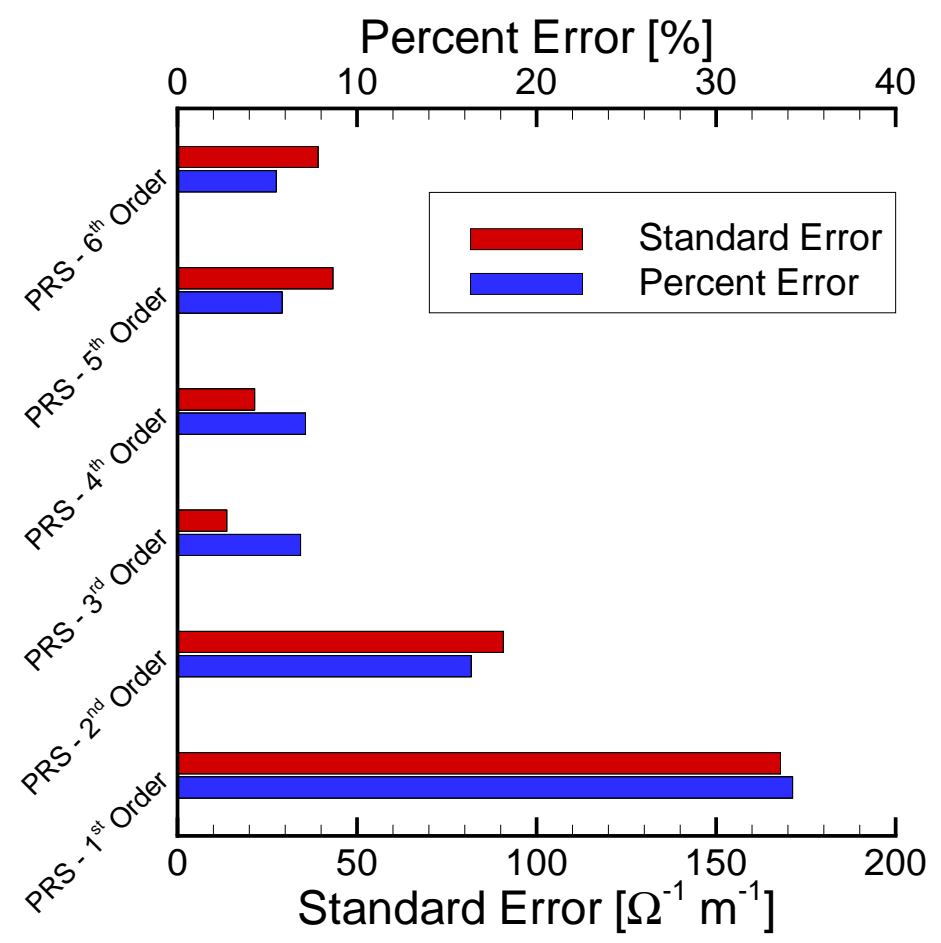

(b) Mean absolute error.

Figure 5.13: Error metrics for several PRS models of weakly-ionized argon. 
differently, this indicator might be important and would have to be evaluated.

If the response was formulated such that the model did predict a negative response, either the model would have to be translated so the minimum value predicted was greater than zero, or a cutoff function would have to be incorporated into the

model. Both methods would degrade the accuracy of the model. While the second option is easy to implement, it may be inappropriate because it introduces a discontinuity into the model that could create instabilities in the coupled MHD solver. Additional ways of improving the conservativeness of a model are discussed in [130].

\subsubsection{Global Sensitivity Analysis}

In an effort to reduce the dimensional requirements of the surrogate model, and consequently, reduce the computational cost of using the model, a global sensitivity analysis is conducted for each model developed using an analysis tool available in SURROGATES Toolbox. The sensitivity analysis results from Monte Carlo simulations of the model to determine the importance of each of its dimensions. Any dimension that has significantly less sensitivity than the others could be removed, thus simplifying the model, without noticeably reducing the model's accuracy. In fact, the accuracy could improve because the number of learning points per subdomain would increase. During the analysis, each of the model's dimensions are modified slightly and used to estimate a response. The difference in the predicted response due to the modification is used to estimate the dimension's sensitivity in the overall model. This procedure is performed thousands of times to reduce the statistical scatter, with high dimensional models requiring more iterations.

Figure 5.14 shows the sensitivity of each dimension, for each of the models developed. The plot suggests the neutral species mole fraction is not important compared 
to the ionic species, except for the case of the $1^{\text {st }}$ order PRS model. Intuitively, this result seems likely since the neutral species dimension is redundant. (Recall that $\left.\chi_{\mathrm{Ar}}=1-\chi_{\mathrm{Ar}^{+}}.\right)$Therefore, five additional models are investigated using reduced dimensions to verify the insignificance of the neutral species for each of the models.

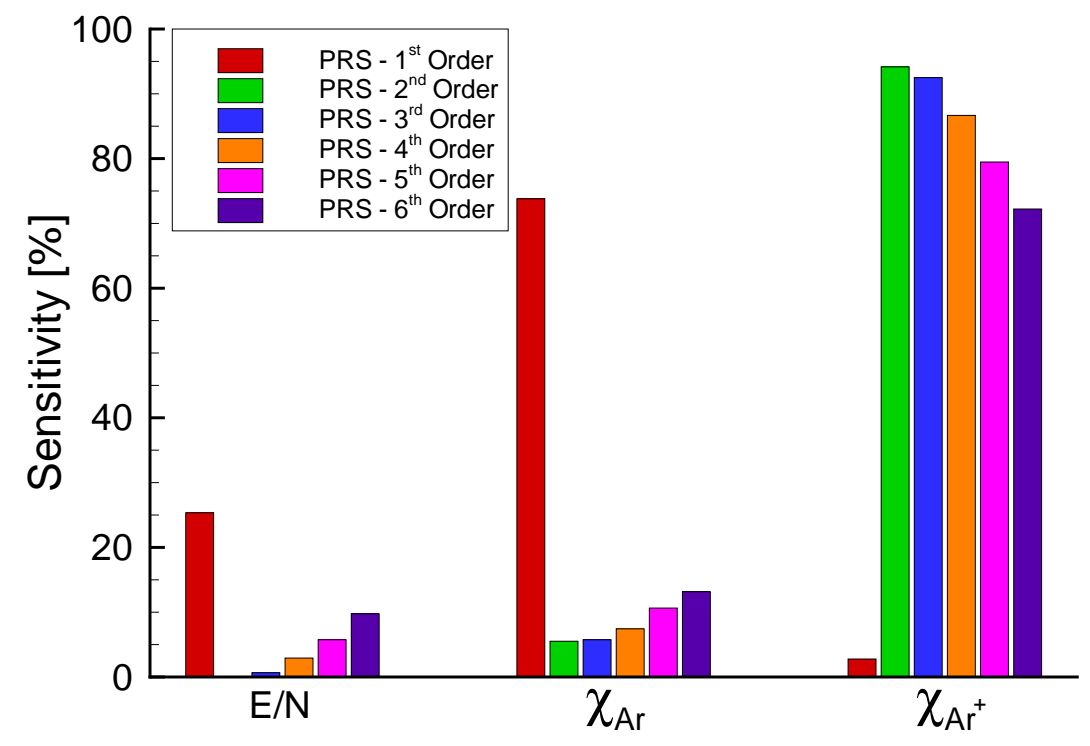

Figure 5.14: Global sensitivity analysis of several surrogate models of weakly-ionized argon using a Monte Carlo technique with fifty thousand iterations.

The reduced dimensional models are determined using the same procedure as the original models, except $\chi_{\mathrm{Ar}}$ is ignored, so the resulting DOE only has two dimensions. Table 5.7 lists the RMSE and MAE using both standard error and percent error for both the original models and the reduced dimensional models due to the global sensitivity analysis. As anticipated, the reduced models are not affected by the missing dimension. In fact, the models appear to perform slightly better. This could be the result of having additional 'learning' points per sub-domain. Recall, the original models had 27.5 points per sub-domain (three-dimensional DOE has 8 sub-domains), while the two-dimensional DOE only has 4 sub-domains or 55 points per sub-domain. 
Table 5.7: Model accuracy for weakly-ionized argon with reduced dimensions. $(\mathrm{E} / N$, $\chi_{\mathrm{Ar}}$, and $\left.\chi_{\mathrm{Ar}^{+}}\right)$

\begin{tabular}{cc||cc|cc}
\hline \hline & \multicolumn{1}{c||}{ Removed } & \multicolumn{2}{c|}{ Error $\left[\Omega^{-1} \mathbf{~ m}^{-1}\right]$} & \multicolumn{2}{c}{ PE [\%] } \\
Model & Dimension(s) & MAE & RMSE & MAE & RMSE \\
\hline PRS $-2^{\text {nd }}$ Order & & 90.7 & 164.1 & 16.3 & 18.3 \\
PRS $-2^{\text {nd }}$ Order & \multirow{2}{*}{$\chi_{\mathrm{Ar}}$} & 90.2 & 163.2 & 16.5 & 18.5 \\
PRS $-3^{\text {rd }}$ Order & & 13.7 & 23.5 & 6.9 & 10.4 \\
PRS $-3^{\text {rd }}$ Order & $\chi_{\mathrm{Ar}}$ & 12.7 & 20.5 & 6.8 & 10.3 \\
PRS $-4^{\text {th }}$ Order & & 21.4 & 42.0 & 7.1 & 10.0 \\
PRS $-4^{\text {th }}$ Order & $\chi_{\mathrm{Ar}}$ & 19.8 & 39.0 & 7.0 & 9.9 \\
PRS $-5^{\text {th }}$ Order & & 43.2 & 84.1 & 5.8 & 7.3 \\
PRS $-5^{\text {th }}$ Order & $\chi_{\mathrm{Ar}}$ & 43.3 & 84.7 & 5.7 & 7.2 \\
PRS $-6^{\text {th }}$ Order & & 39.1 & 76.2 & 5.5 & 6.8 \\
PRS $-6^{\text {th }}$ Order & $\chi_{\mathrm{Ar}}$ & 39.1 & 76.2 & 5.5 & 6.8 \\
\hline \hline
\end{tabular}

\subsubsection{Computational Cost Analysis}

Because the electrical conductivity model will ultimately be coupled to a flow solver which may evaluate the electrical conductivity model billions of times for a single simulation, (e.g., a typical three-dimensional simulation has two million fluid cells and requires five thousand iterations to achieve convergence [131]), the computational cost of using a specific model is an important factor to consider when choosing the best model to implement. Since each model developed is based on the PRS model, the higher-order models have many more coefficients, and as such, have a greater computational cost.

In order to estimate the expected computational cost of using a specific model, each model was implemented into the flow solver then used to determine the conductivity in 10,000 fluid cells. The number of cells selected is within an order of 
magnitude of the number of cells per processor in a typical three-dimensional simulation conducted using this flow solver [132]. The timing exercise is repeated several times to reduce scatter in the results due to background tasks being performed on the computing system. Figure 5.15 shows the resulting data points for each model.

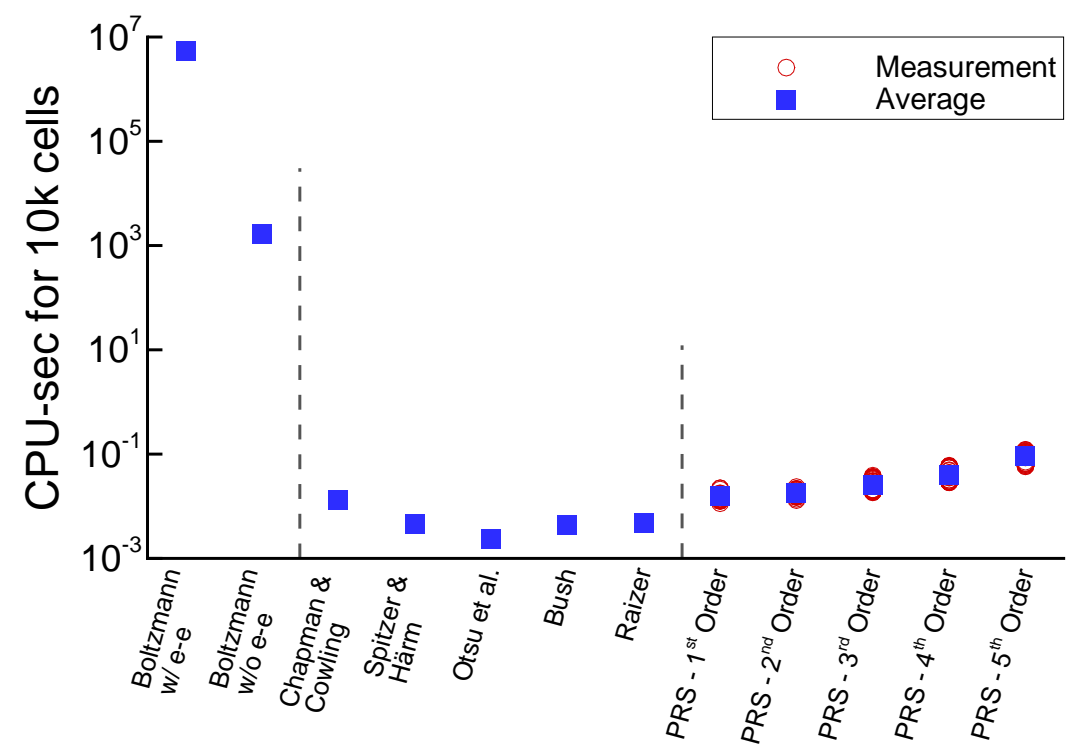

Figure 5.15: Average CPU-cost for executing various electrical conductivity models.

Overall, the PRS models have similar CPU-costs compared to the existing semiempirical models, which make it possible to recover most of the accuracy of the Boltzmann solutions without the expense of directly coupling the Boltzmann solver to the fluid-MHD code. However, the higher order PRS models do have a slightly higher CPU-cost, which is a direct result of the larger number of constant coefficients in the model. In the case of the three dimensional model of weakly-ionized argon, the difference in the total number of coefficients is relatively small between a $3^{\text {rd }}$ order model (20 coefficients), and a $6^{\text {th }}$ order model (84 coefficients), however the difference is much more dramatic for higher dimensional models (e.g., 11 species air). 


\subsubsection{Summary of Argon Models}

Overall, the various PRS models are able to capture the behavior of the solutions to Boltzmann's equation over a large design space. Although the $3^{\text {rd }}$ through $6^{\text {th }}$ order models appear to produce similar results, the $3^{\text {rd }}$ order model has the lowest standard error. Since the model is formulated using the natural logarithm function, all predictions made by the model are positive, which is an important characteristic of the model since the electrical conductivity of a gas cannot be negative. Very little difference is observed by removing the $\chi_{\text {Ar }}$ dimension as suggested by the global sensitivity analysis, so there is an opportunity to minimize the CPU-cost of the model, but the margin is minimal because the original model only has three dimensions and is accurately represented using a $3^{\text {rd }}$ order model. In addition, the timing study demonstrates very little computational cost difference between the models, although the higher order models do have a slightly higher CPU-cost. Considering all these factors, it appears the most suitable model to implement is the $3^{\text {rd }}$ order PRS model developed for three dimensions. Additional information about the dimensions of the model and the constant coefficients is available in Appendix F.

\subsection{Surrogate Models of Air}

Since hypersonic flows in air are the main focus of this thesis, it is necessary to also develop an electrical conductivity model for 11 species air. The procedure is identical to that outlined above for argon, except the DOE has eleven dimensions. As with weakly-ionized argon, the model is formulated using the natural logarithm to ensure a positive prediction (response) from the model, as seen in Eqn. 5.18: 


$$
\ln \left(\frac{\alpha}{\sigma}\right)=f\left(\mathrm{E} / N, \chi_{\mathrm{N}_{2}}, \chi_{\mathrm{O}_{2}}, \chi_{\mathrm{NO}}, \chi_{\mathrm{N}}, \chi_{\mathrm{O}}, \chi_{\mathrm{N}_{2}^{+}}, \chi_{\mathrm{O}_{2}^{+}}, \chi_{\mathrm{NO}^{+}}, \chi_{\mathrm{N}^{+}}, \chi_{\mathrm{O}^{+}}\right)
$$

Since there are so many dimensions of the DOE, it is computationally prohibitive to compute solutions to more 'learning' and 'testing' points than are absolutely necessary. Following the recommendations outlined in Section 5.3.2, 4,096 'learning' points (2 points per sub-domain) and 3,072 'testing' points (1.5 points per sub-domain) are computed while allowing for electron-electron collisions. These computations are performed by computing individual solutions of the Boltzmann equation in parallel with grid computing. Since there are eleven dimensions, the sample points in the DOE are shown in Fig. 5.16 in each of the eleven dimensions. So, one point in one of the sub-frames corresponds to a point in each of the other sub-frames.

A majority of the sample points are clustered at low values for the neutral species $\left(\chi_{\mathrm{N}_{2}}, \chi_{\mathrm{N}_{2}}, \chi_{\mathrm{O}_{2}}\right.$, etc. $)$, because the summation of all the mole fractions must equal one, so if a sample point has a high concentration of a single neutral species, all the other species must be small. As a result, all neutral species cluster near low concentrations. In addition, the placement of the sample points in the DOE is accomplished automatically by the HLHC tool in SURROGATES Toolbox, which attempts to maximize the distance between all the sample points in all dimensions of the DOE.

The accuracy metrics are populated in Fig. 5.17(a) for the RMSE and in Fig. 5.17(b) for the MAE using both the standard and percent error. Similar to weaklyionized argon, the $1^{\text {st }}$ and $2^{\text {nd }}$ order polynomial models perform poorly compared to the $3^{\text {rd }}$ order and higher models. However, unlike the weakly-ionized argon, the $3^{\text {rd }}$ order model does not appear to have the lowest error. This could be due to the 

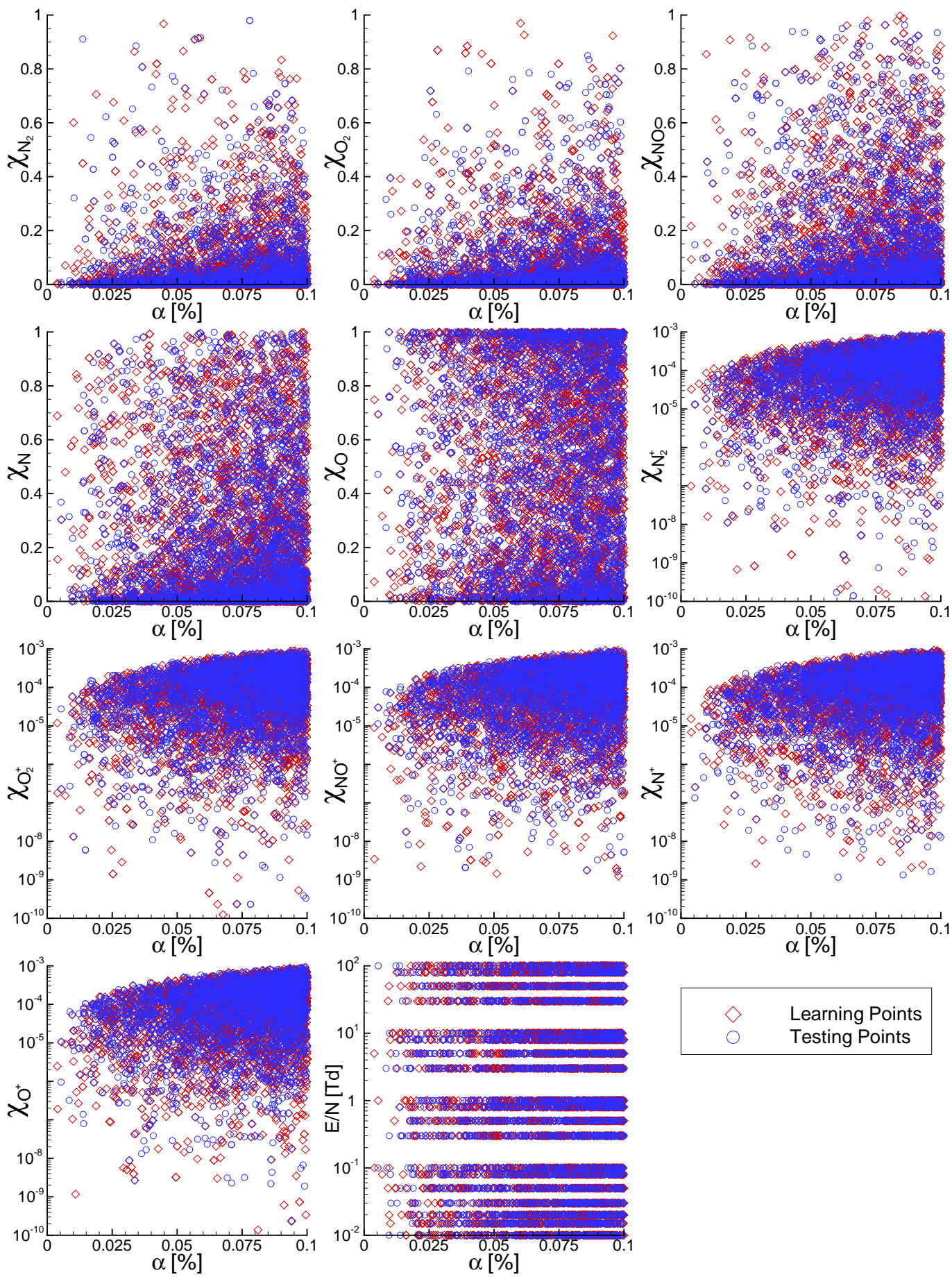

Figure 5.16: Design of Experiment 'learning' and 'testing' points for the elevendimensional surrogate model of weakly-ionized air. 
minimal number of 'learning' points used, or that the PRS models simulated do not have enough extrema to fully capture the behavior of the solution surface across the selected dimensions.

Since the eleven species air model may be suffering from insufficient learning points, a global sensitivity analysis is conducted to identify which variables are less important, as seen in Fig. 5.18. Unlike the weakly-ionized argon, the species fractions are not directly related. As such, it is less apparent that a specific species could be removed, although, consistent with argon, the ionized species seem to be more important.

Another important factor to consider is the cost of using a specific PRS model. Table 5.3 shows that the number of coefficients for a $3^{\text {rd }}$ order polynomial with 11 dimensions has 364 constant coefficients, whereas a $4^{\text {rd }}$ order model with 11 dimensions has 1,365 constant coefficients. Since the higher order model has almost four times as many coefficients, its CPU-cost should be about four times more expensive.

\subsubsection{Constricted Design Space}

Although the model developed has relatively low error, it may be possible to improve the model by constricting the design space. By reducing the size of the DOE, the number of 'learning' points per volume of the DOE increases. Since the model is for weakly-ionized air, the mole fraction of nitrogen molecule $\mathrm{N}_{2}$, should not be greater than $80 \%$. In addition, the mole fraction of molecular oxygen should be less than $21 \%$. Standard air contains very little oxygen in any other form (i.e., $\mathrm{NO}, \mathrm{CO}_{2}$, etc.), so another obvious restriction is that the atomic oxygen mole fraction is less than twice the maximum of molecular oxygen $\left(\chi_{\mathrm{O}} \leq 42 \%\right)$. Therefore, a restricted

design space is created by imposing: $\chi_{\mathrm{N}_{2}} \leq 0.85, \chi_{\mathrm{O}_{2}} \leq 0.25$, and $\chi_{\mathrm{O}} \leq 0.5$. The rest 


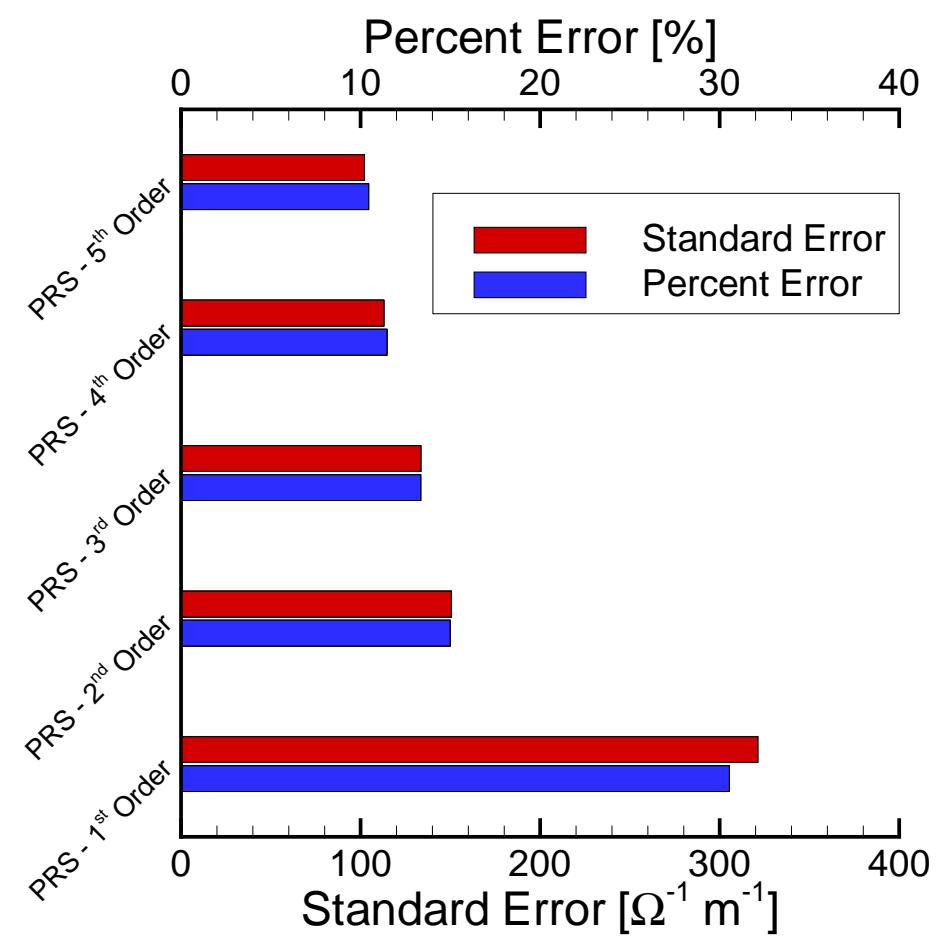

(a) Root mean square error.

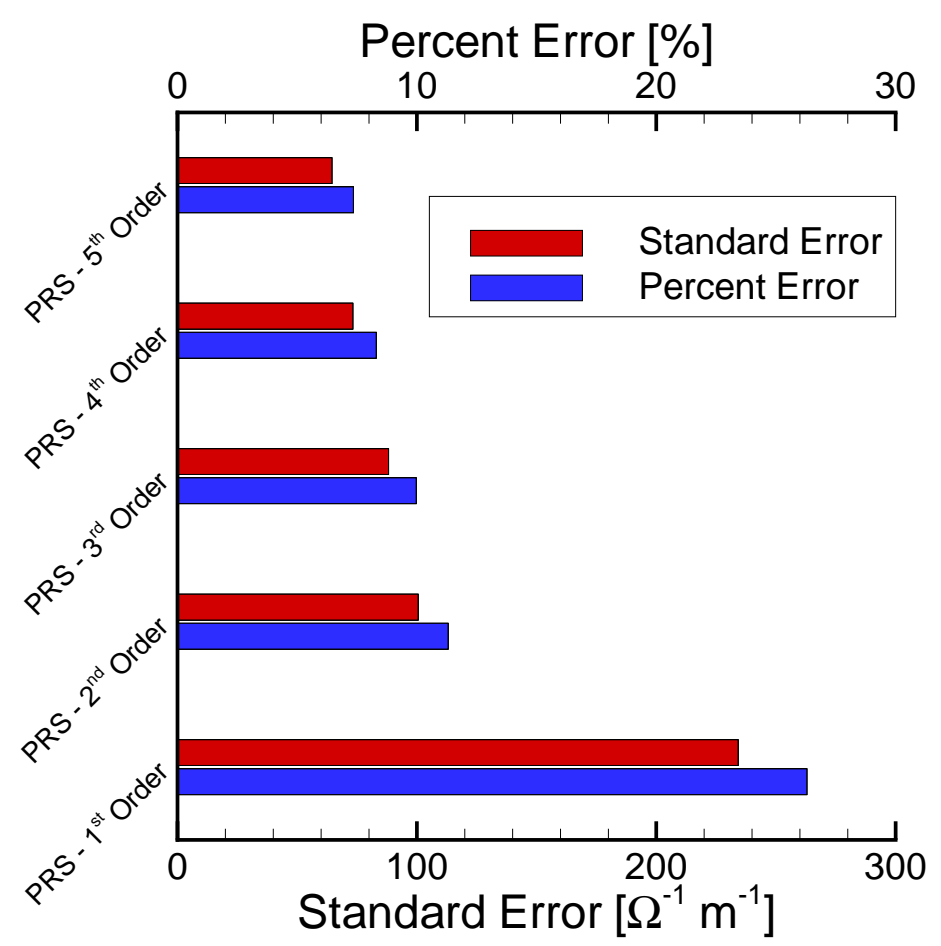

(b) Mean absolute error.

Figure 5.17: Error metrics for several PRS models of weakly-ionized air. 


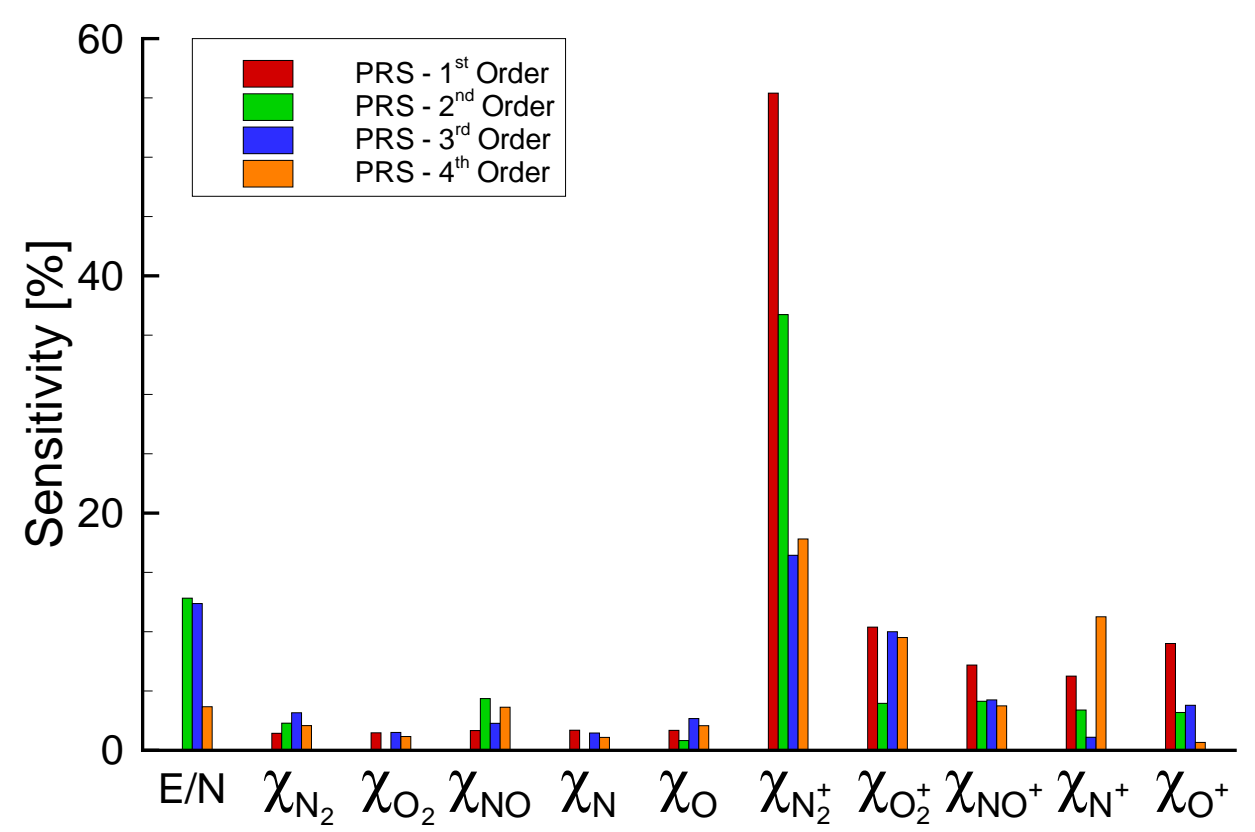

Figure 5.18: Global sensitivity analysis of several surrogate models of weakly-ionized air using a Monte Carlo technique with fifty thousand iterations.

of the design space is unadjusted (i.e., $10^{-2} \mathrm{Td} \leq \mathrm{E} / N \leq 10^{2} \mathrm{Td}$, $\chi_{\text {neutrals }} \leq 100 \%$, $\chi_{\text {ions }} \leq 0.1 \%$ ). These restrictions are evident in the new DOE, as seen in Fig. 5.19.

However, even with the restricted DOE, the models do not perform better than the results achieved with the full design space as seen in Table 5.8.

These results reenforce the possibility that the comparatively low order PRS models with respect to the number of dimensions (i.e., a $3^{\text {rd }}$ order model for 11 dimensional DOE versus a $3^{\text {rd }}$ order model for a 3 dimensional DOE), are insufficient to fully capture the behavior of the surface produced by the Boltzmann solver. This explanation is plausible since the electrical conductivity of a gas becomes very large as its degree of ionization becomes high. Since the degree of ionization is a result of each ionic species (and a combination of them), it is probable that local extrema exist along each of the ionic species dimensions. The number of extrema in a polynomial is equal to its order minus one [133] (i.e., a $3^{\text {rd }}$ order polynomial 

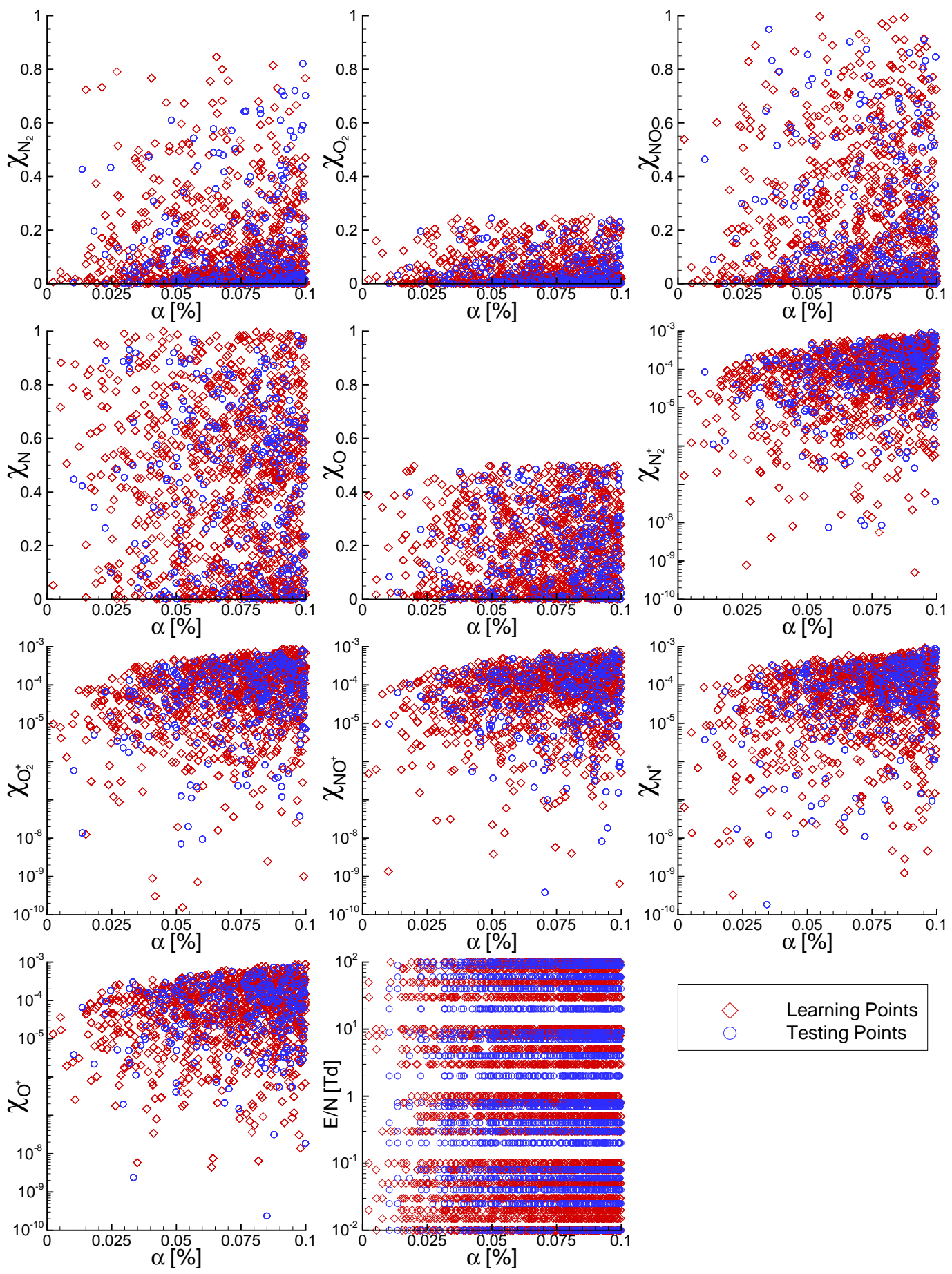

Figure 5.19: Design of Experiment 'learning' and 'testing' points for the elevendimensional surrogate model of weakly-ionized air in a reduced design space. 
Table 5.8: Model accuracy for weakly-ionized air with and without a reduced design space.

\begin{tabular}{cc||cc|cc}
\hline \hline & \multirow{2}{*}{ Design } & \multicolumn{2}{|c|}{ Error } & {$\left[\Omega^{-1} \mathbf{m}^{-1}\right]$} & \multicolumn{2}{c}{ PE [\%] } \\
PRS model & Space & MAE & RMSE & MAE & RMSE \\
\hline $1^{\text {st }}$ & full & 234.1 & 321.2 & 26.3 & 30.5 \\
$1^{\text {st }}$ & reduced & 236.1 & 313.7 & 27.8 & 31.9 \\
$2^{\text {nd }}$ & full & 100.5 & 150.6 & 11.3 & 15 \\
$2^{\text {nd }}$ & reduced & 101.8 & 158.6 & 11 & 14.8 \\
$3^{\text {rd }}$ & full & 88.2 & 133.5 & 10 & 13.3 \\
$3^{\text {rd }}$ & reduced & 93.1 & 144.7 & 10 & 13.6 \\
$4^{\text {th }}$ & full & 73.2 & 112.9 & 8.3 & 11.5 \\
$4^{\text {th }}$ & reduced & 75.3 & 119.8 & 8.3 & 11.6 \\
$5^{\text {nd }}$ & full & 64.4 & 102.1 & 7.3 & 10.4 \\
$5^{\text {th }}$ & reduced & 66.9 & 106.1 & 7.4 & 10.4 \\
\hline \hline
\end{tabular}

has 2 extrema). This means that PRS models of less than $6^{\text {th }}$ order may not have enough extrema to capture the peak electrical conductivity along each of the five ionic species dimensions.

In addition, the reduced DOE results imply that PRS models developed for the full DOE are sufficiently accurate and cannot be significantly improved by removing part of the design space, particularly since the removed parts of the design space exist in regions of primarily low ionization (e.g., $100 \% \mathrm{~N}_{2}$ would have no ionziation).

\subsubsection{Summary of Air Models}

Overall, it appears the PRS models developed are able to capture most of the behavior seen by the solutions to Boltzmann's equation. The higher order models do provide better accuracy, but the significant increase in CPU-cost associated with 
using the higher order models is disproportionate to the gains. Therefore, either the $2^{\text {nd }}$ or $3^{\text {rd }}$ order PRS model is best suited to be implemented. In fact, it may be

most logical to start the simulations using the faster $2^{\text {nd }}$ model and then switch to the $3^{\text {rd }}$ order model to refine the solution. The constant coefficients for each model are listed in Appendix F.

\subsection{Surrogate Models - Applied}

The importance of using an accurate estimate for the electrical conductivity is illustrated by exploring the effects a magnetic field has on a weakly-ionized flow. This concept, also known as an MHD-Heat Shield, relies on the magnetic force (Lorentz force) to push the bow shock further from the vehicle and decelerate the hot ionized particles as they approach the stagnation point. Because the focus of this exercise is to illustrate the importance of the electrical conductivity on the magnetic force, for now the Hall effect is ignored. This is a poor assumption since the magnetic field strength is large and the typical densities are low, but it reduces the electrical conductivity tensor to a scalar. With no electrodes present, the electric field is small, so the electromagnetic power deposition is neglected $\mathbf{j} \cdot \mathbf{E}=0$. The current density simplifies to $\mathbf{j}=\sigma(\mathbf{u} \times \mathbf{B})$, and the magnetic force is: $\mathbf{j} \times \mathbf{B}=\sigma(\mathbf{u} \times \mathbf{B}) \times \mathbf{B}$.

The same blunt-nose elliptic cone geometry $(L=3 \mathrm{~m})$ simulated in Section 3.4.1 is used along with the freestream conditions listed in Table 3.3, which is equivalent to Mach 12.6 air at an altitude of $40 \mathrm{~km}$. An 11 species finite rate air chemistry model is employed with temperature contours illustrated in Fig. 5.20.

A dipole magnetic field is employed, with its centroid located inside the blunted tip of the vehicle $0.14 \mathrm{~m}$ from the stagnation point along the $x$-axis. Since the magnetic field strength decays as $1 / r^{3}$, where $r$ is the radius from the centroid, the 


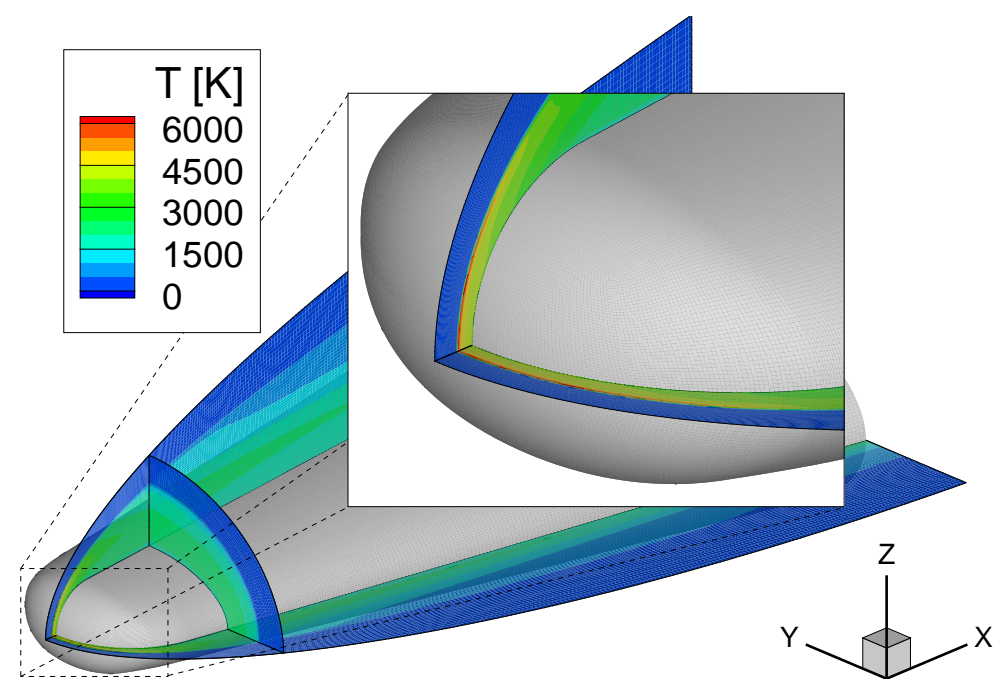

Figure 5.20: Temperature contours for Mach 12.6 air flow around a blunt-nose elliptic cone geometry $(L=3 \mathrm{~m})$.

peak magnetic field strength is located along the stagnation line, with a simulated peak strength of 0.2 Tesla (2000 Gauss), as seen in the magnetic field contours of Fig. 5.21.

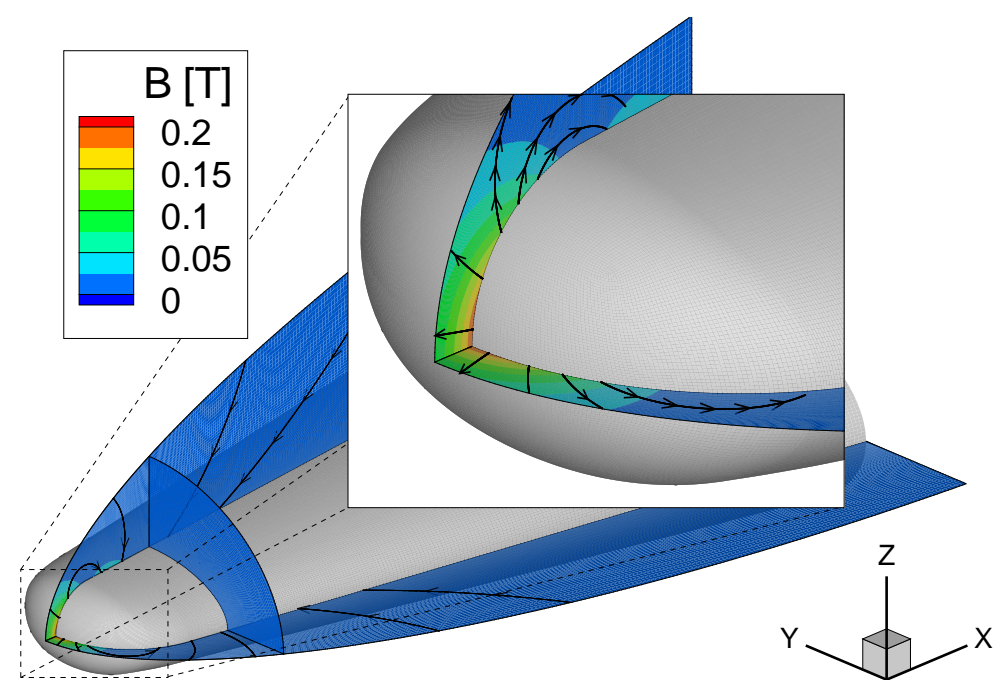

Figure 5.21: Magnetic field contours and streamlines for a blunt-nose elliptic cone geometry with a dipole magnet.

Figures 5.22(a), 5.22(b) and 5.22(c) plot the resulting electrical conductivity contours for the flow-field using the Raizer, Chapman and Cowling, and $2^{\text {nd }}$ order PRS 
models, respectively. Consistent with the assumptions listed previously, the electric field is assumed negligible, $\mathbf{E}=0$. The PRS model contours have a magnitude about half that predicted by Raizer's model, while the profile it predicts is similar to the Chapman and Cowling model. Because Raizer's model is only dependent on temperature, it predicts peak conductivity to occur near the bow shock, which then falls sharply within the stagnation region. This behavior is not observed with the other models, which show the conductivity remaining relatively high within the stagnation region due to an increase in the degree of ionization.

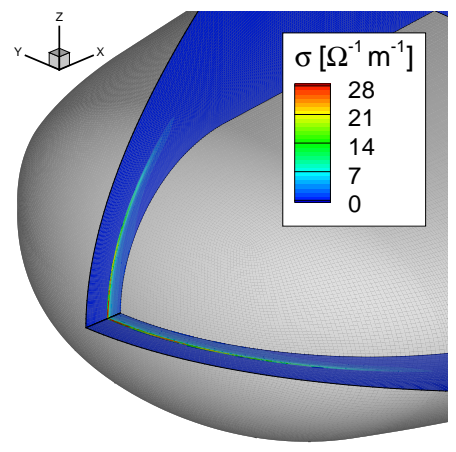

(a) Raizer.

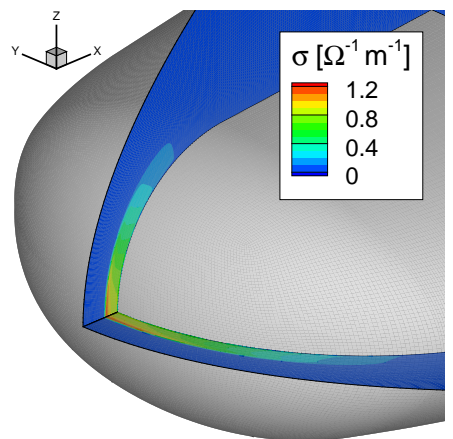

(b) Chapman and Cowling.

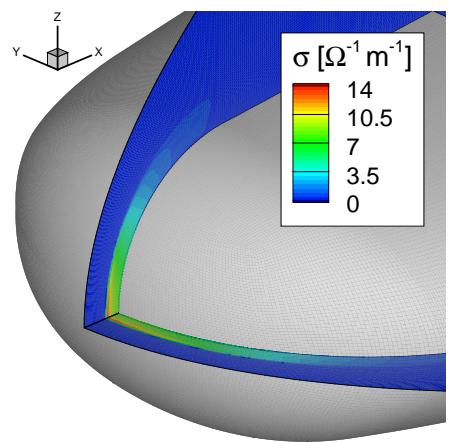

(c) $2^{\text {nd }}$ order PRS.

Figure 5.22: Electrical conductivity contours for Mach 12.6 air flow around the stagnation region of a blunt-nose elliptic cone geometry $(L=3 \mathrm{~m})$, for various conductivity models. $(\mathbf{E}=0)$

Since the magnetic force acting on the flow is directly proportional to the value and location of the electrical conductivity, the different electrical conductivity models lead to significantly different forces being generated. The magnetic force $(\mathbf{j} \times \mathbf{B})$ contours for each model with the dipole magnetic field are illustrated in Fig. 5.23. Asymmetry is observed in the magnetic force contours because of the elliptical geometry.

Although the magnetic field strength decays as $1 / r^{3}$ from its centroid, the significantly higher electrical conductivity within the shock layer, as estimated by Raizer's 


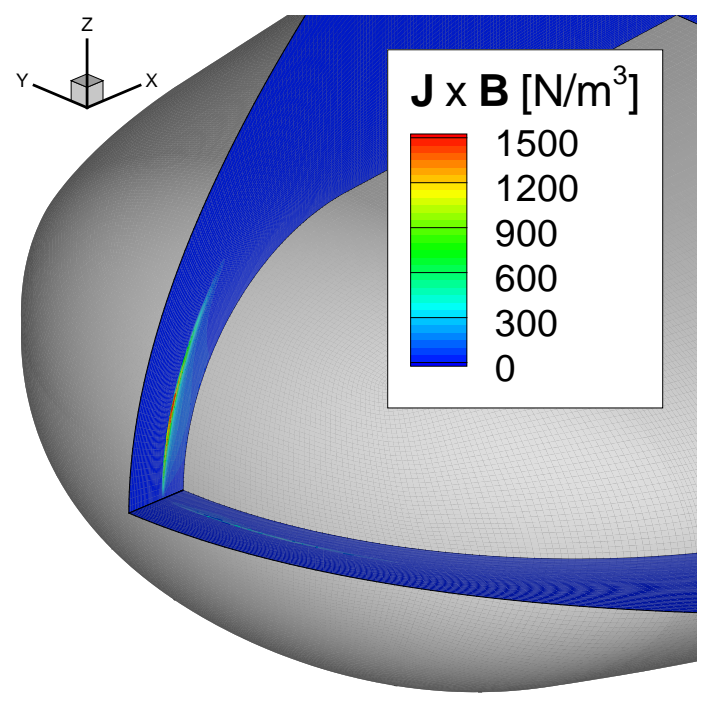

(a) Raizer.

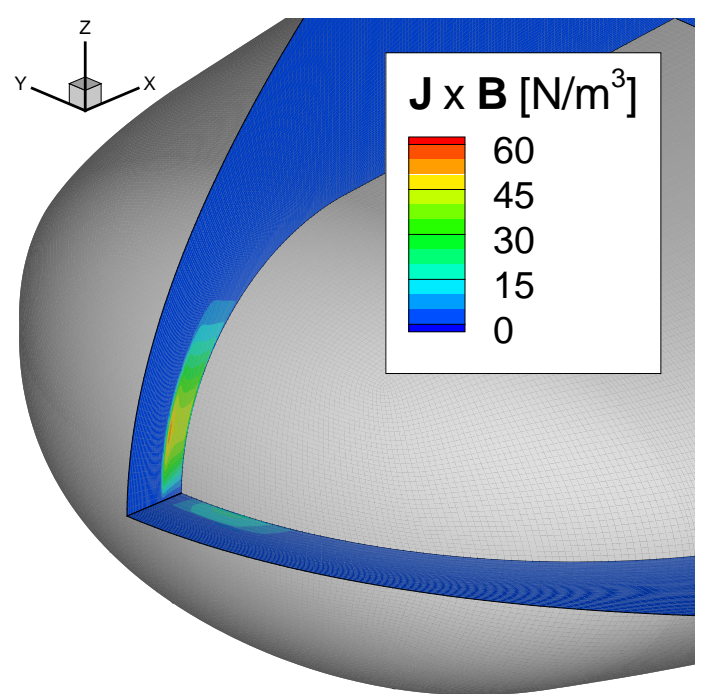

(b) Chapman and Cowling.

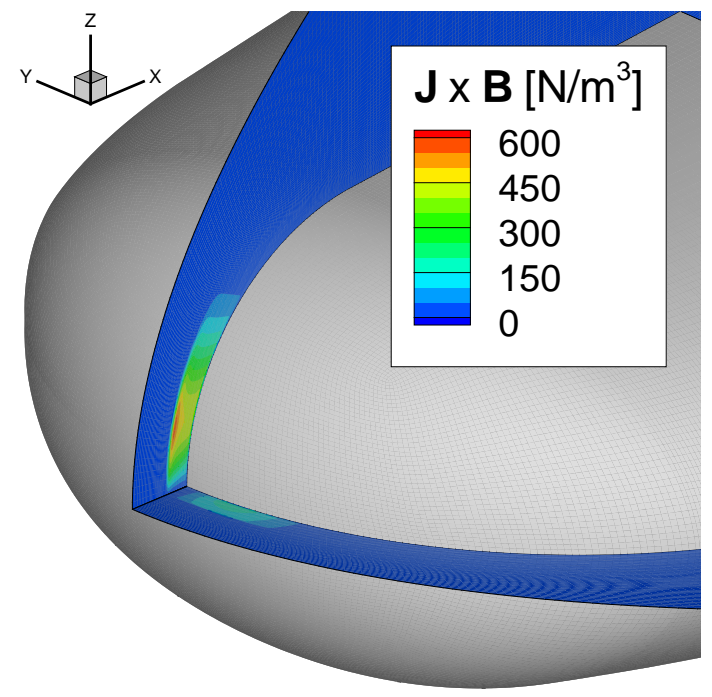

(c) $2^{\text {nd }}$ order PRS.

Figure 5.23: Magnetic force contours for Mach 12.6 air flow around the stagnation region of a blunt-nose elliptic cone geometry $(L=3 \mathrm{~m})$, for various conductivity models with a $0.2 \mathrm{~T}$ peak strength dipole magnetic field. 
model, results in a peak magnetic force about twenty times greater than that estimated using the Chapman and Cowling model. However, integrating the magnetic force over the volume shows that the PRS model introduces the greatest average force into the system, as seen in Table 5.9.

Table 5.9: Total magnetic force produced by a $0.2 \mathrm{~T}$ dipole magnet field centered in a blunt-nose elliptic cone geometry $(L=3 \mathrm{~m})$, in March 12.6 air.

\begin{tabular}{ccc}
\hline \hline Model & $\sigma_{\max }\left[\Omega^{-1} \mathbf{m}^{-1}\right]$ & Magnetic Force $[\mathbf{N}]$ \\
\hline Raizer & 28.4 & 0.1755 \\
Chapman and Cowling & 1.2 & 0.0218 \\
$2^{\text {nd }}$ Order PRS & 13.4 & 0.2253 \\
\hline \hline
\end{tabular}

As seen in Fig. 5.23, the magnetic force is significantly influenced by the electrical conductivity model selected. In addition to changing the overall force added to the flow, the magnetic force contours vary between models, which will lead to other, downstream effects. Therefore, it is vital to use an accurate electrical conductivity model when computing electromagnetic effects.

\subsection{Conclusions}

Although simple models for the electrical conductivity provide reasonable predictions within their reference range, they are not general and are only valid within that range. This is an obstacle for off-design simulations and evaluation of plasmabased devices. The added benefit of determining the conductivity directly using a coupled Boltzmann solver does provide additional generality to the flow solver and improves physical modeling of the interactions, but is too computationally expensive for practical use on even the most modern parallel computing hardware. Therefore, this chapter outlined a procedure to develop a suitable surrogate model to solutions 
of Boltzmann's equation.

Surrogate models of solutions to Boltzmann's equation were developed for a large design space and evaluated based on their accuracy and computational cost. In addition, reduced dimensional models were evaluated using coordinate transformation and global sensitivity analysis in order to develop polynomial models of electrical conductivity for eleven species air and weakly-ionized argon. A $3^{\text {rd }}$ order Polynomial Response Surface PRS, was found to be sufficiently accurate for predicting 3 species weakly-ionized argon, while maintaining the computational cost of other semi-empirical models. The procedure was then repeated to develop $2^{\text {nd }}$ and $3^{\text {rd }}$ order PRS models for 11 species air. The $2^{\text {nd }}$ order model was applied to a representative vehicle employing the MHD-parachute concept to further illustrate the importance of using an appropriate electrical conductivity model when accounting for electromagnetic effects. 


\section{CHAPTER VI}

\section{Computational Analysis of MHD Applications}

This chapter utilizes the tools developed in the previous chapters to explore two different plasma-based flow control devices. The first part of the chapter is an investigation of an arc discharge over a blunt leading-edge geometry without the presence of an external magnetic field. The second part explores the use of a magnetic field as a heat shield (i.e., to reduce surface heating), on a nonconducting surface. Each scenario explores the different extremes of electromagnetic effects. The arc discharge scenario only depends on electric fields, while the second scenario primarily relies on magnetic fields. Both cases involve three-dimensional flows and require accurate modeling of the electrical conductivity.

\subsection{Arc Discharge}

In Chapter III, the effects of localized heat addition were explored and quantified. However, the study did not specify how the energy (heat) was transfered into the domain. One method of depositing the energy is by using a glow discharge, which is the result of electrons moving between two electrodes with a potential difference, as seen in Fig. 6.1.

A glow discharge relies on electron impact ionization, which corresponds to high 


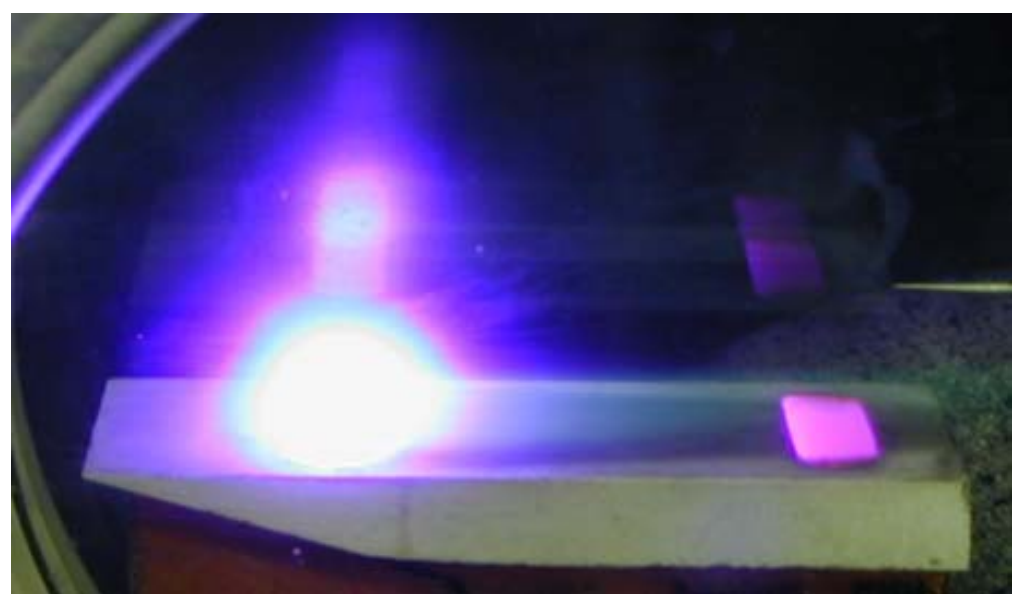

Figure 6.1: Glow discharge on a flat plate in Mach 5.2 air flow with a potential difference of $2800 \mathrm{~V}$ (50 mA). Image from Kimmel et al. [28].

imposed potential with low current. An arc discharge uses the same transport mechanism as a glow discharge (i.e., electrons moving between electrodes), except that the temperature and the current are much higher due to the thermal ionization of the gas. An arc discharge deposits thermal energy into the flow via Joule heating. In this scenario, hypersonic air flows over a three-dimensional blunt leading-edge geometry, which was previously discussed in Section 4.4.2. Joule heating $(\mathbf{j} \cdot[\mathbf{E}+\mathbf{u} \times \mathbf{B}])$, occurs in the domain because of the potential difference specified between two electrodes located on the geometry, as seen in Fig. 6.2.

The freestream conditions are consistent with Mach 12.6 air at $40 \mathrm{~km}$, as listed in Table 3.3. The leading edge profile and freestream conditions are selected to produce a strong bow shock, which is beneficial for this investigation because the post shock temperature in the stagnation region is sufficiently high to partially ionize the flow (i.e., natural ionization). The simulations are run using eleven species chemically reacting air $\left(\mathrm{N}_{2}, \mathrm{O}_{2}, \mathrm{NO}, \mathrm{N}, \mathrm{O}, \mathrm{N}_{2}^{+}, \mathrm{O}_{2}^{+}, \mathrm{NO}^{+}, \mathrm{N}^{+}, \mathrm{O}^{+}\right.$, and e). The gridindependence study, presented in Section 4.4.2, found adequate similarity between solutions obtained on the 'fine' and 'very fine' grids for both the nondimensional 


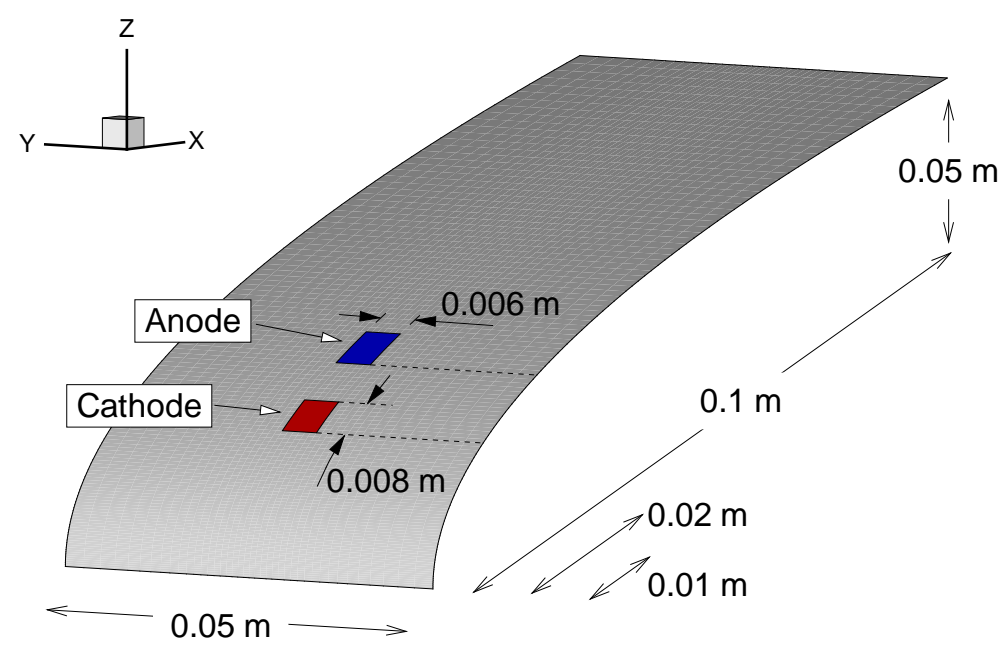

Figure 6.2: Geometry and placement of flush-mounted electrodes for a power-law shaped $\left(z=x^{0.5}\right)$, blunt leading-edge geometry.

heat flux and pressure coefficient, so the 'fine' grid is assumed sufficiently refined and used in the rest of the analysis. The 'fine' grid contains 120 nonuniform axial points, 60 nonuniform radial points, and 40 nonuniform points along the width of the body, such that grid clustering occurs near the stagnation point, along the geometry surface, and near the centerline (near the electrodes).

Figure 6.3 shows the temperature contours for thermal nonequilibrium flow over the blunt leading-edge geometry. The temperature contours show that the peak translational temperature $\mathrm{T}$, is $1000 \mathrm{~K}$ higher than the vibrational-electron-electronic temperature $\mathrm{T}_{v e}$, verifying the necessity of assuming thermal nonequilibrium in the simulations.

Without an applied magnetic field, only the conservation of energy equations are affected by the arc discharge. In addition, Ohm's law reduces to $\mathbf{j}=\sigma \mathbf{E}$, and the Hall effect is absent, which means the electrical conductivity tensor reduces to a scalar. (Recall from Section 4.5: $\beta=e \mathrm{~B} / m_{\mathrm{e}} \nu_{m}$.) The only component of the total energy deposition is the Joule heating, so the total energy source term reduces to: 


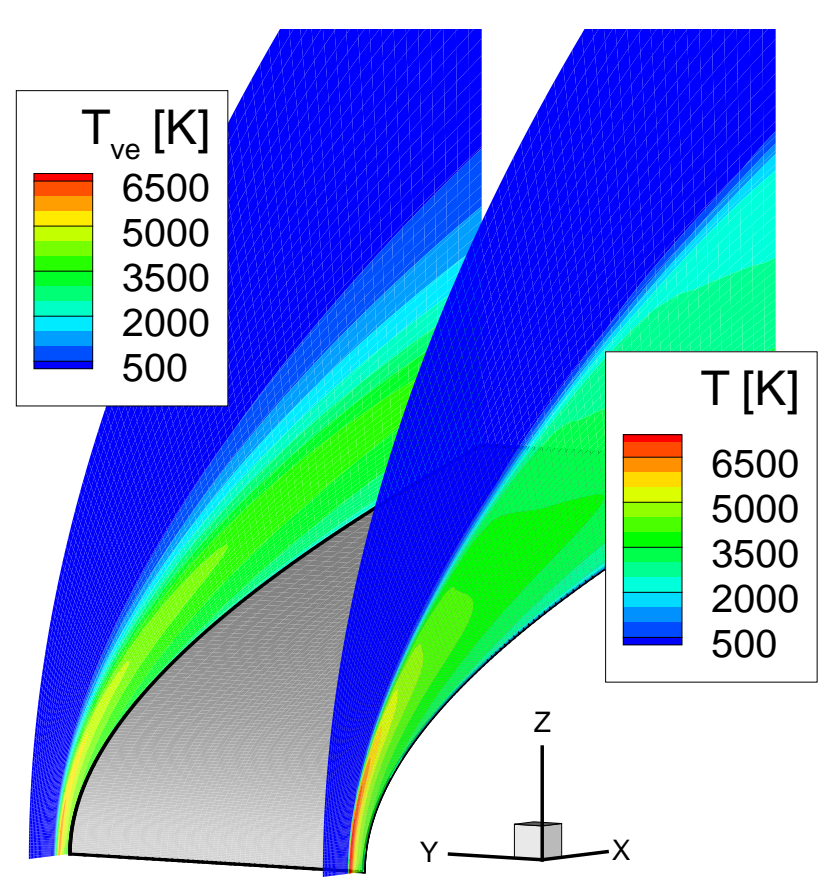

Figure 6.3: Thermal nonequilibruim temperature contours for Mach 12.6 air flow around a blunt leading-edge geometry.

$\mathbf{j} \cdot \mathbf{E}=\sigma \mathbf{E}^{2}$. The constant $\gamma$ in the Joule heating term of the vibrational-electronelectronic energy equation $\left(\gamma \mathbf{j} \cdot[\mathbf{E}+\mathbf{u} \times \mathbf{B}]=\gamma \sigma \mathbf{E}^{2}\right)$, is assumed to be $\gamma=0.75$ based on similar computation studies by Shang and Surzhikov [134].

The cathode is set with an imposed (negative) voltage, while the anode is set slightly positive, so the current travels from the anode to the cathode, as seen in Fig. 6.4. Similar results were obtained by Poggie for a glow discharge on a flat plate [135]. Although the current travels from the anode to the cathode, some additional current comes into the domain through the side boundaries because of the high level of electrical conductivity that exists in the shock (which spans the width of the domain). The high electrical conductivity in the shock is due to the model selected (Raizer's conductivity model is only a function of temperature).

The current density flowing through the cathode is computed and multiplied by the cathode area to determine the total current at the cathode. Since the poten- 


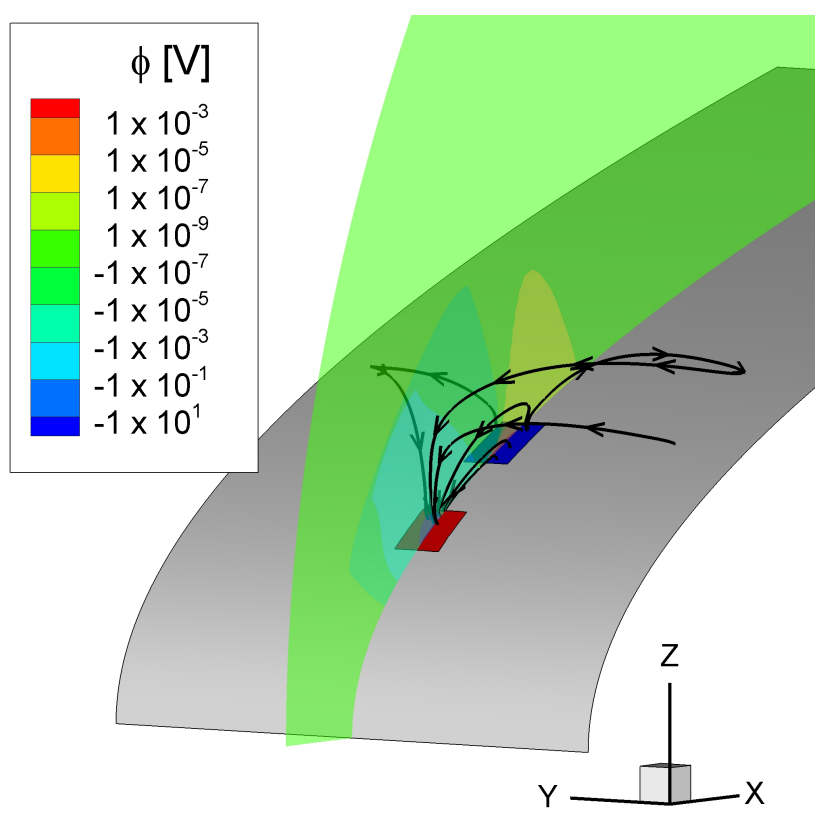

Figure 6.4: Electric potential contours and current lines for flow between flushmounted electrodes with a $10 \mathrm{~V}$ potential difference for Mach 12.6 air flow around in a blunt leading-edge geometry. (Raizer's conductivity model).

tial is specified at the cathode, the expected power deposition is estimated using a combination of Joule's and Ohm's laws:

$$
P=I V
$$

where $P$ is the power, $I$ is the current, and $V$ is the voltage. The total rate of heat added to the flow due to the arc discharge is computed by integrating the Joule heating term over the domain $\left(\sigma \mathbf{E}^{2}\right)$. The expected power deposition and the actual power deposition in the simulation (due to Joule heating) are plotted for several voltages, as shown in Fig. 6.5.

As seen in the figure, the actual energy deposition is less than expected $(\sim 89 \%$ efficiency), which may be a result of inadequate numerical resolution. The power deposition efficiency decreases as the voltage increases, suggesting a smaller potential 


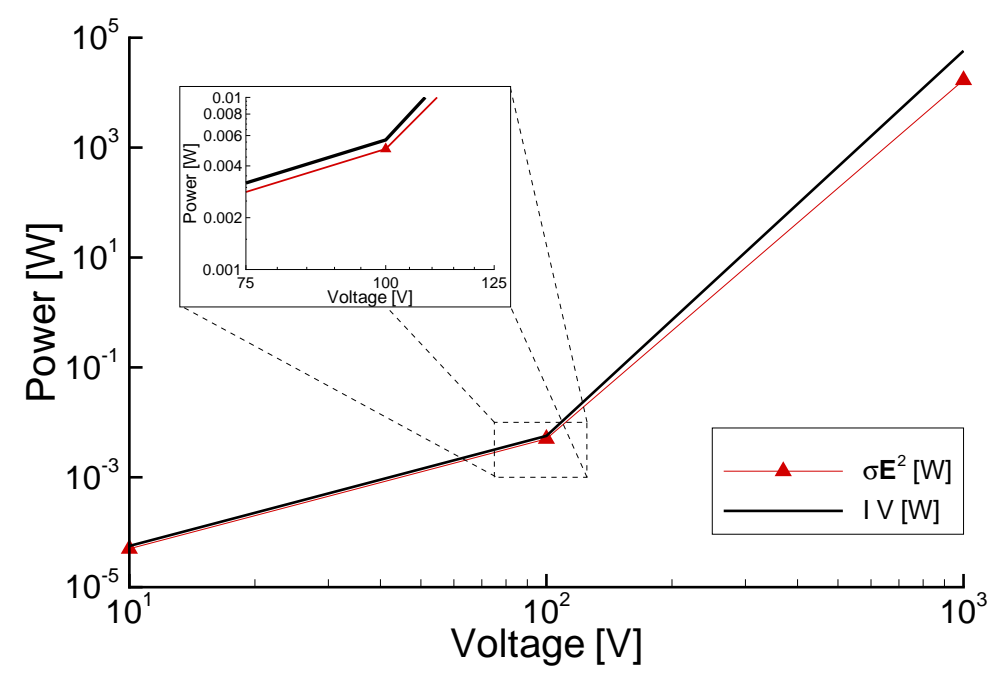

Figure 6.5: Thermal energy deposition due to an applied voltage between a cathode and anode flush-mounted in a blunt leading-edge geometry. (Raizer's conductivity model)

difference is more efficient. However, the low voltage scenario also has a low current, which may not be strong enough to initiate an arc between the electrodes.

\subsection{MHD-Heat Shield}

This section explores the feasibility of using a strong electromagnet, producing a dipole magnetic field, located within the forebody of a hemisphere capped body as an effective means of heat transfer reduction at its surface. This concept is also known as an MHD-Heat Shield, since the heat transfer reduction is the result of a magnetic force that works to oppose, slow, and deflect the ionized portion of the incoming flow. Decelerating the flow increases the shock standoff distance, which reduces wall-normal gradients, and thus the heat flux, particularly to the stagnation point.

An implementation of the stagnation point heat reduction concept can be seen in Fig. 6.6, which shows infrared photographs of a recent MHD-Heat Shield experiment 
conducted by Gülhan et al. [30]. The darker region near the center of the hemisphere capped forebody of Fig. 6.6(b) corresponds to a decrease in luminosity (i.e., a decrease in temperature, with may correspond to a reduction in heat transfer). The deceleration of the flow also increases the drag on the vehicle, which may further reduce heat transfer in flight (MHD-parachute concept).

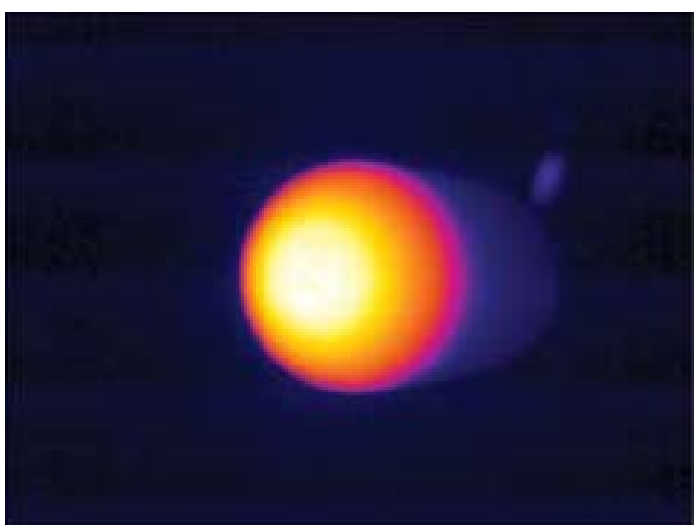

(a) Without an applied magnetic field.

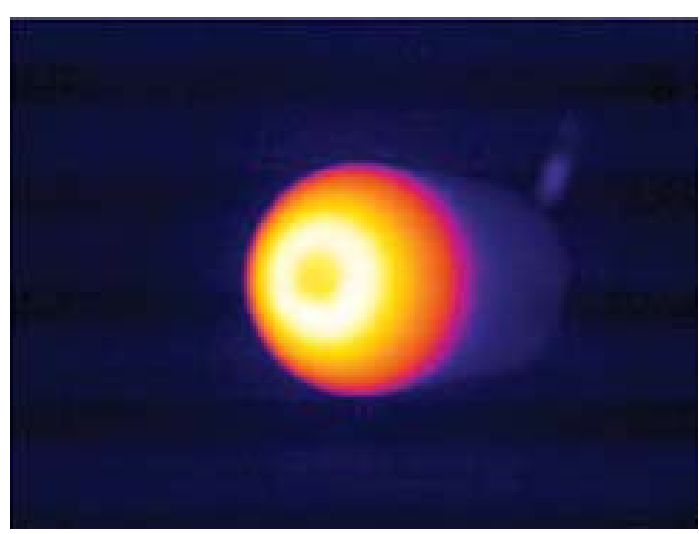

(b) With an applied magnetic field.

Figure 6.6: Infrared photographs of hemisphere capped geometry with and without an applied-magnetic field. Image from Gülhan et al. [30].

Three-dimensional calculations are carried out for Mach 4.75 argon flow over a hemisphere capped forebody attached to a cylinder, which was originally studied experimentally by Kranc et al. [16]. The forebody hemisphere has a radius of 1.5 inch $\left(\mathrm{r}_{\mathrm{n}}=0.0381 \mathrm{~m}\right)$, and the geometry is mounted parallel to the freestream, as seen in Fig. 6.7. The freestream flow is composed of strongly ionized argon (the degree of ionizion was estimated by Kranc et al., as $\alpha=0.025)$, which is produced by a plasma torch (direct-current arc-heater). The heater is located before the convergingdiverging nozzle, which accelerates the gas into the test chamber. Kranc et al. state that the electrons are 'frozen' in the nozzle, and that the flow is not chemically reacting after it is initially ionized by the heater. The flow conditions reported by Kranc et al. are listed in Table 6.1. 


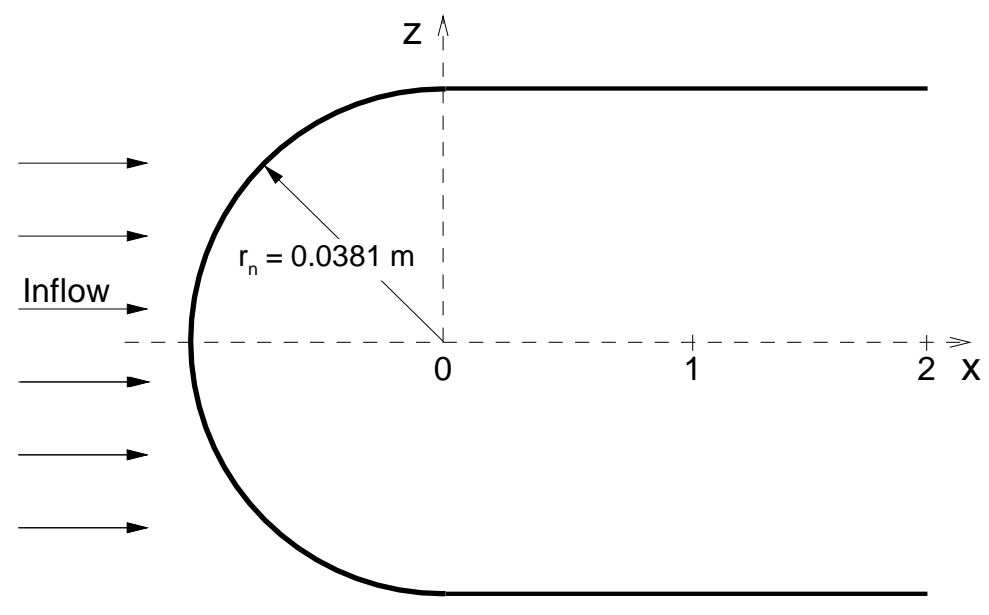

Figure 6.7: Hemisphere capped geometry. Adapted from [16].

Table 6.1: Flow conditions for the MHD-Heat Shield experiment as reported by Kranc et al. [16].

\begin{tabular}{crl}
\hline \hline Parameter & Value & \\
\hline $\mathrm{M}$ & 4.75 & \\
$u_{\infty}$ & 3000.0 & $\mathrm{~m} / \mathrm{s}$ \\
$\mathrm{T}_{\infty}$ & $1100.0 \mathrm{~K}$ \\
$\mathrm{~T}_{w}$ & $300.0 \mathrm{~K}$ \\
$\mathrm{p}_{\infty}$ & 27.8 & $\mathrm{~Pa}$ \\
$\rho_{\infty}$ & $1.035 \times 10^{-4}$ & $\mathrm{~kg} / \mathrm{m}^{3}$ \\
$n_{\mathrm{e}}$ & $4 \times 10^{19}$ & $\mathrm{~m}{ }^{-3}$ \\
$\alpha$ & 0.025 & \\
$\mathrm{r}_{\mathrm{n}}$ & 0.0381 & $\mathrm{~m}$ \\
$\mu_{\infty}$ & $8 \times 10^{-5}$ & $\mathrm{~kg} / \mathrm{m} \cdot \mathrm{s}$ \\
$\operatorname{Re}_{x}$ & 3880 & $\mathrm{~m}{ }^{-1}$ \\
$\operatorname{Re}_{L}$ & 148 & \\
\hline \hline
\end{tabular}


A structured grid is generated, but is decomposed into two grid domains because of the hemispherical forebody. The first domain includes the forebody, while the second accommodates the rest of the geometry. While the baseline flow solution (the flow without the magnetic field), is axisymmetric, the rest of the simulations are computed using a three-dimensional grid because the MHD routine developed in Chapter IV is currently only implemented for three-dimensional domains.

The grid is generated with equal spacing along the hemisphere portion of the geometry (first domain), and gradually increases in spacing along the remaining surface (second domain). Grid points are equally spaced around the circumference of the geometry and the radial points are algebraically spaced to increase the number of points close to the body. As a result, cell clustering occurs primarily in the hemispherical forebody and near the body surface. The baseline grid uses 50 points along the body (30 in the hemispherical region), 30 points along one quarter of the circumference, and 30 radial points. Two refined grids are also developed and used in the grid-independence study, giving the following set of computational meshes: $50 \times 30 \times 30($ coarse $)$, to $100 \times 60 \times 60($ medium $)$, to $200 \times 120 \times 120$ (fine).

Chemically non-reacting, thermodynamic equilibrium simulations are computed using the variable hard sphere (VHS) viscosity model. The VHS model is used because the viscosity is assumed to only be a function of temperature, since the species present (argon, argon ion, and electrons), have a single energy mode and are chemically non-reacting:

$$
\mu=\mu_{\text {ref }}\left(\frac{T}{T_{r e f}}\right)^{\omega}
$$

where $\mu$ is the viscosity, the reference viscosity coefficient, $\mu_{r e f}=2.117 \times 10^{-5} \mathrm{~N} \mathrm{~s} / \mathrm{m}^{2}$, for a reference temperature, $\mathrm{T}_{\text {ref }}=273 \mathrm{~K}$, and a viscosity index, $\omega=0.81$. This 
method, as outlined by Schwartzentruber et al. [136], requires several reference coefficients which are listed in [108].

Figure 6.8 plots the pressure coefficient and nondimensional heat flux for the baseline flow along the surface of the geometry, as defined in Eqns. 2.7 and 2.9, respectively. The grid-independence study shows little difference between the 'medium' and 'fine' grids, therefore the 'medium' grid is considered sufficiently refined and is used in the rest of the analysis. However, the MHD module might require its own grid resolution study.

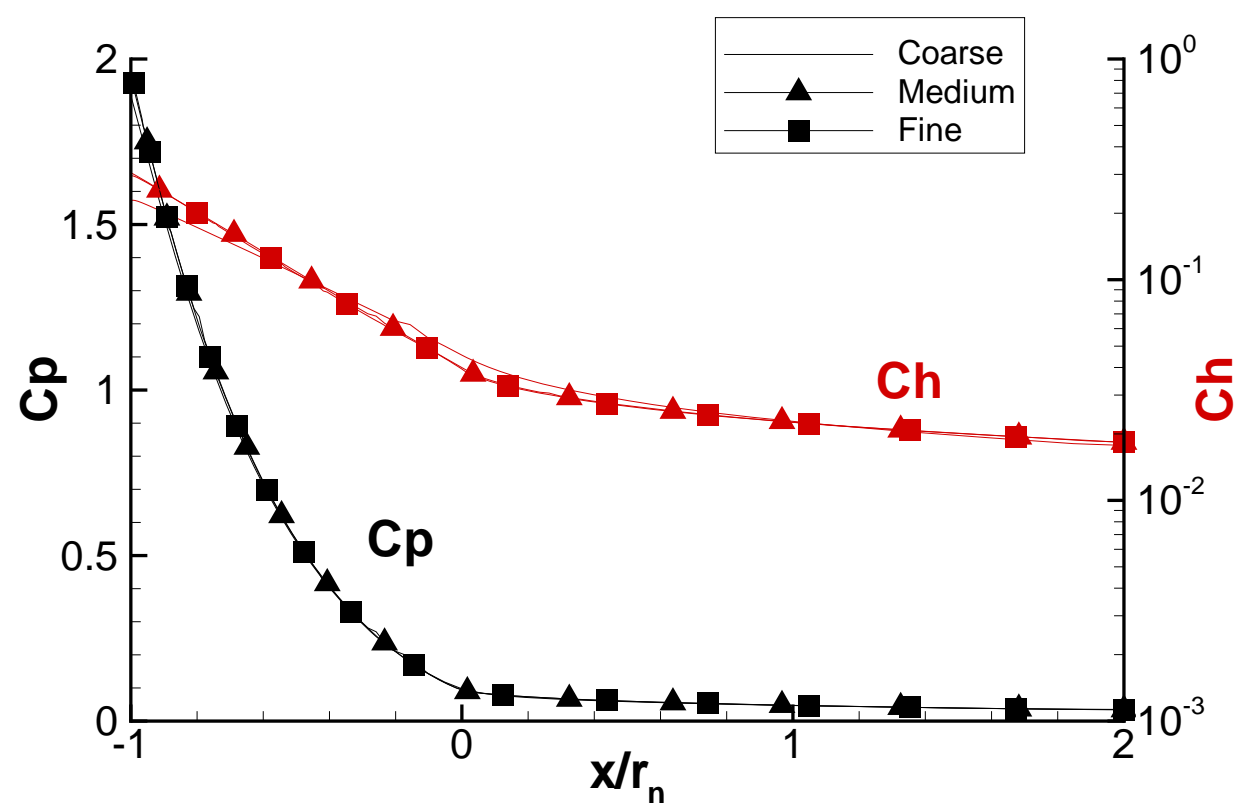

Figure 6.8: Nondimensional pressure and heat flux along the surface of Mach 4.75 argon flow around a hemisphere capped geometry for various grids.

A closer examination of the freestream conditions, specifically, the degree of ionization, reveals $\alpha$ was estimated by Kranc et al. using tables from Arave and Huseley [137], along with the stagnation temperature and pressure. While this approach may be approximately correct, the degree of ionization is better estimated by using the Saha equation for a singly ionized atomic gas [138]: 


$$
\frac{\alpha^{2}}{1-\alpha^{2}} p=3.16 \times 10^{-7} \mathrm{~T}^{5 / 2} \exp \left(-\frac{\varepsilon_{i}}{k \mathrm{~T}}\right)
$$

where $p$ is the pressure in atmospheres, $\mathrm{T}$ is the temperature in Kelvin, Boltzmann's constant is $1.3807 \times 10^{-23} \mathrm{~J} / \mathrm{K}$, and $\varepsilon_{i}$ is the ionization energy required to remove the electron from the atom in the gas considered. The ionization potential for argon, $\varepsilon_{i}=2.53 \times 10^{-18} \mathrm{~J}$, and the stagnation pressure and temperature are $0.49 \mathrm{~atm}$ and $9700 \mathrm{~K}$, respectively. Using the Saha equation yields a degree of ionization $\alpha=0.00623$.

This new estimate for the degree of ionization only changes two values listed in Table 6.1, namely, $\alpha=6.23 \times 10^{-3}$ and $n_{\mathrm{e}}=10^{19} \mathrm{~m}^{-3}$, which results in a slight modification to the freestream conditions, as seen in Table 6.2.

Table 6.2: Modifications to the freestream conditions for the MHD-Heat Shield experiment of Kranc et al. [16].

\begin{tabular}{c|cc}
\hline \hline & \multicolumn{2}{|c}{ Value } \\
Parameter & Reported & Adjusted \\
\hline$u_{\infty}[\mathrm{m} / \mathrm{s}]$ & 3000 & 3000 \\
$\mathrm{~T}_{\infty}[\mathrm{K}]$ & 1100 & 1100 \\
$\mathrm{~T}_{w}[\mathrm{~K}]$ & 300 & 300 \\
$\alpha$ & 0.025 & 0.00623 \\
$\rho_{\mathrm{Ar}}\left[\mathrm{kg} / \mathrm{m}^{3}\right]$ & $1.01 \times 10^{-4}$ & $1.09 \times 10^{-4}$ \\
$\rho_{\mathrm{Ar}}+\left[\mathrm{kg} / \mathrm{m}^{3}\right]$ & $2.65 \times 10^{-6}$ & $6.85 \times 10^{-7}$ \\
$\rho_{\mathrm{e}}\left[\mathrm{kg} / \mathrm{m}^{3}\right]$ & $3.64 \times 10^{-11}$ & $9.41 \times 10^{-12}$ \\
\hline \hline
\end{tabular}

Since the changes to the individual species densities are minimal, and the flow is assumed chemically non-reacting, these slight adjustments to the freestream conditions are assumed not to noticeably alter the resulting flow-field, so the 'medium' grid discussed previously is assumed to provide sufficient grid-independence and is 
used in the rest of the analysis. The remaining simulations reported in this chapter use the freestream conditions corresponding to $\alpha=0.00623$.

Figure 6.9 plots the temperature contours for the flow without the magnetic field. As seen in the figure, the peak temperature is $9000 \mathrm{~K}$, which is $150 \mathrm{~K}$ hotter than the solution computed using the freestream conditions corresponding to $\alpha=0.025$. The temperature increase is the result of the slight increase in total density. Using the baseline flow-field solutions from both simulations (i.e. $\alpha=0.025$ and 0.00623 ), the expected range of electrical conductivity, estimated from for several models presented in Chapter V, is displayed in Table 6.3. The results indicate slight discrepancies in estimated electrical conductivities, with the $2^{\text {nd }}$ order PRS predictions residing between the semi-empirical models.

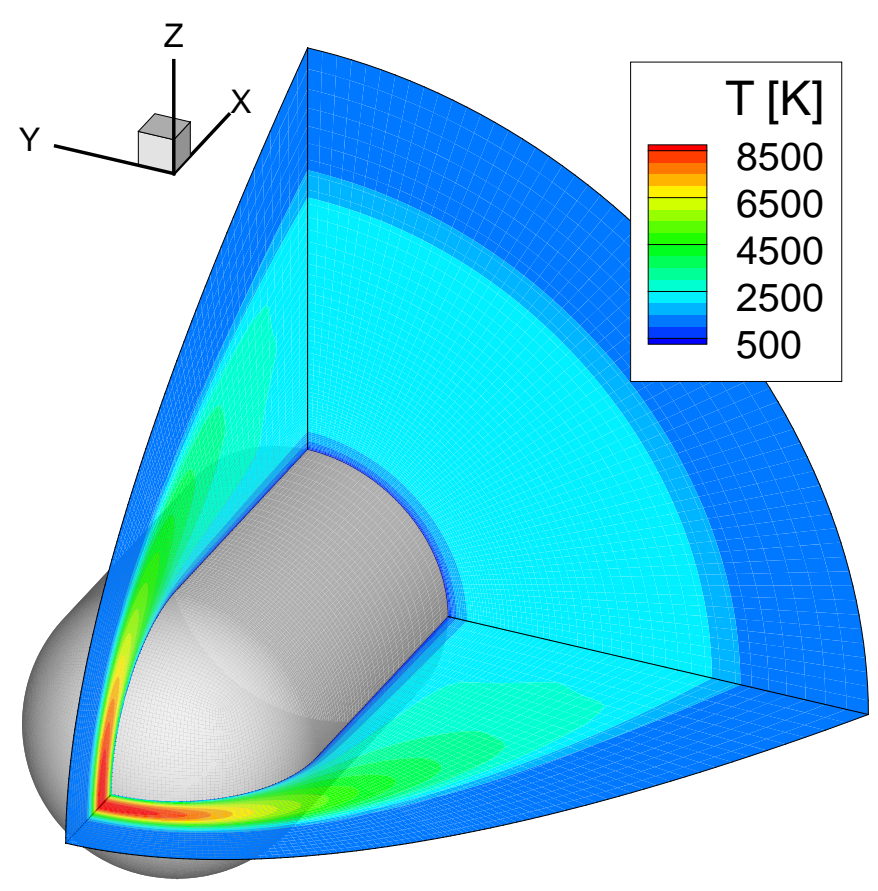

Figure 6.9: Temperature contours for Mach 4.75 argon flow around a hemisphere capped geometry. $(\alpha=0.00623)$

In the experiment of Kranc et al., the applied magnetic field is produced by an electromagnet located inside the hemisphere capped forebody, whose magnetic field 
Table 6.3: Electrical conductivity estimates for the MHD-Heat Shield experiment without an applied magnetic field. $(\mathrm{E} / N=0)$

\begin{tabular}{c|cc}
\hline \hline$\sigma\left[\Omega^{-1} \mathbf{c m}^{-1}\right]$ & $\alpha=0.025$ & $\alpha=0.00623$ \\
\hline Raizer & $0-1.4$ & $0-1.5$ \\
Chapman and Cowling & $17.7-50.4$ & $4.4-24.2$ \\
$2^{\text {nd }}$ order PRS & $0.1-0.2$ & $7.1-8.1$ \\
\hline \hline
\end{tabular}

can be approximated by a dipole. The magnetic field decays as $\mathrm{r}^{-3}$ from its centroid, which is assumed to be located along the $x$-axis, where the forebody merges with the rest of geometry $\left(\mathrm{x} / \mathrm{r}_{\mathrm{n}}=0\right)$, as seen in Fig. 6.10. The magnetic field contours are nondimensionalized by the peak magnetic field strength $B_{\max }$ in the flow, which occurs at the stagnation point $\left(\mathrm{x} / \mathrm{r}_{\mathrm{n}}=-1\right.$ for this configuration). Note that the peak magnetic field strength is used to designate each simulation for the rest of this analysis.

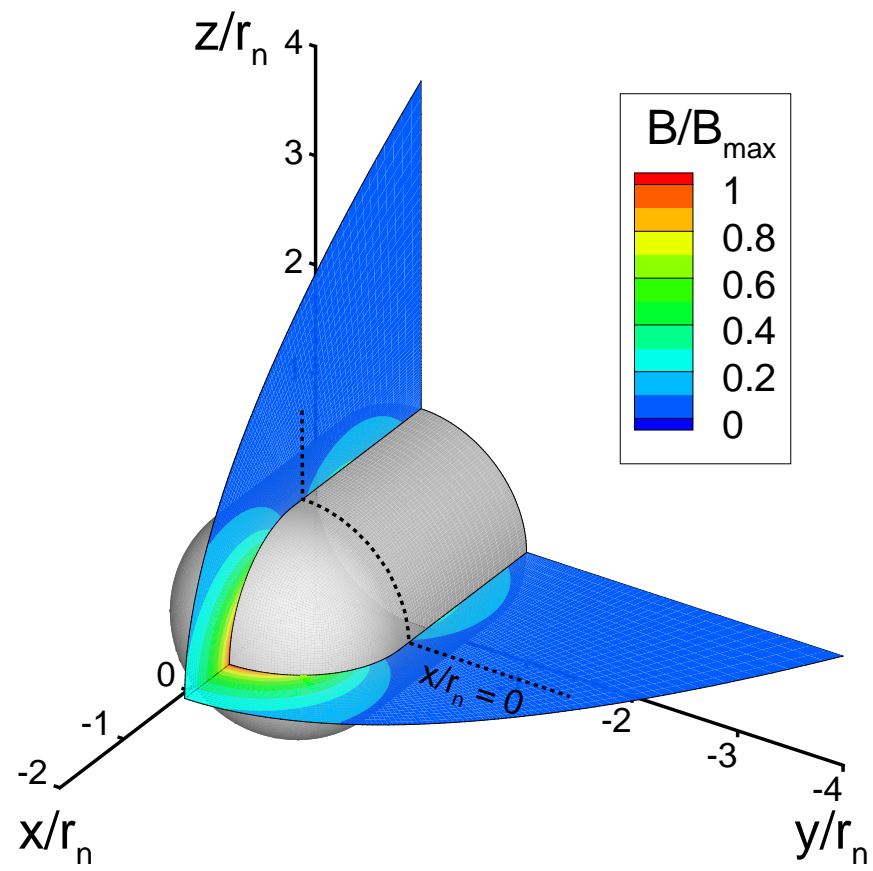

Figure 6.10: Nondimensional dipole magnetic field contours from a magnet located in the hemisphere capped geometry. 
In Cartesian coordinates, the dipole magnetic field is:

$$
\mathbf{B}=\mathrm{B}_{\max } \frac{\cos \theta}{2} \frac{\mathrm{r}_{\mathrm{n}}^{3}}{\mathrm{r}^{5}}\left[\begin{array}{c}
\mathrm{r}^{2}-x^{2} \\
-3 x y \\
-3 x z
\end{array}\right]
$$

where the angle $\theta=\arcsin \left(\sqrt{y^{2}+z^{2}} / \mathrm{r}\right)$.

The flow-field around the geometry (without the applied magnetic field), is axisymmetric and steady, as evident in the temperature contours seen in Fig. 6.9. This means the electric current must only travel in the azimuthal direction (perpendicular to the incoming flow, around the axis of symmetry), and the electric field must be zero [10]. This reduces the magnetic force in the momentum equation to $\tilde{\sigma} \cdot(\mathbf{u} \times \mathbf{B}) \times \mathbf{B}$, and sets the energy deposition term in the total energy equation to zero, $\mathbf{j} \cdot \mathbf{E}=0$. Note that Joule heating is still present under these assumptions, $\gamma(\mathbf{E}+\mathbf{u} \times \mathbf{B}) \cdot \mathbf{j} \neq 0$. Since the electric field is assumed zero and the magnetic field is applied, only the current density field $(\mathbf{j}=\tilde{\sigma} \cdot[\mathbf{u} \times \mathbf{B}])$, needs to be updated in the MHD module.

Simulations are carried out for several magnetic field strengths and electrical conductivity models. The simulations start from the steady-state 'baseline' solution (without an applied magnetic field), and iterate until the flow-field has achieved a new steady-state (converged). Steady-state is assumed once the L2 residual error from the conservation equations decays to the minimum allowed by machine precision zero, as seen in Fig. 6.11 for a typical simulation. In this scenario, at least 10 characteristic flow times worth of time steps are required to achieve a steady-state flow-field solution. A characteristic flow time is defined as the time it takes for the flow to traverse the length of the geometry (i.e. flow time $=L / u_{\infty}$ ).

The assumption that the electric field is negligible $(\mathbf{E}=0)$, is verified by sim- 


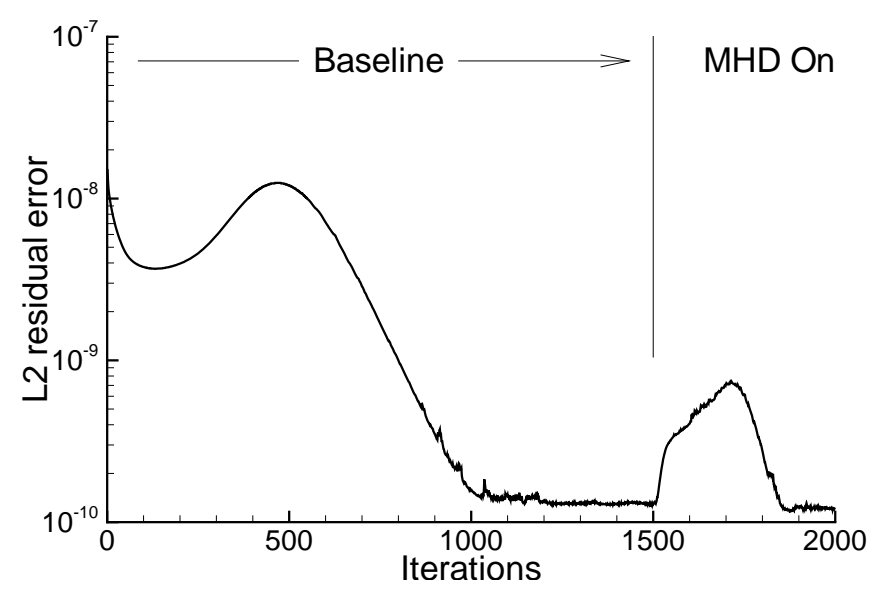

Figure 6.11: L2 residual error from a simulation of Mach 4.75 argon flow around a hemisphere capped geometry with a $0.13 \mathrm{~T}$ magnet. (Chapman and Cowling conductivity model)

ulating the MHD-Heat Shield with and without computing the electric field. The Chapman and Cowling electrical conductivity model is employed for both simulations with $\mathrm{B}_{\max }=0.28 \mathrm{~T}$. The MHD module is used to update the electric field every 5 fluid iterations. Figure 6.12 plots the temperature contours and current lines for both scenarios. As seen in the figures, computing $\mathbf{E}$ from the MHD module does not alter the flow structure or current lines.

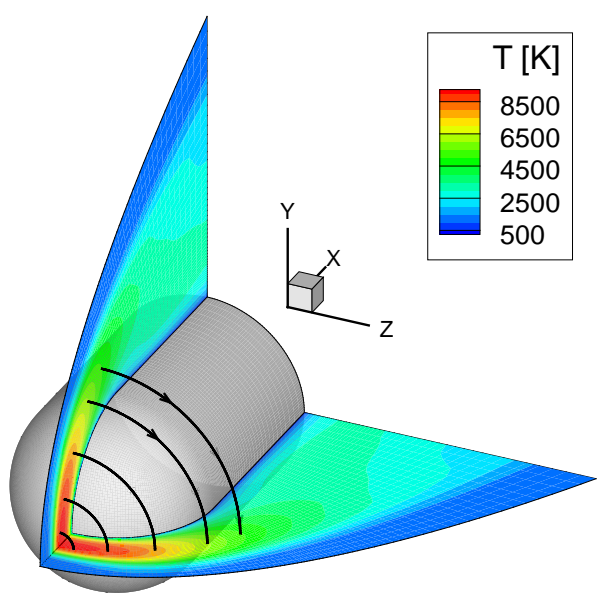

(a) Assuming $\mathbf{E}=0$.

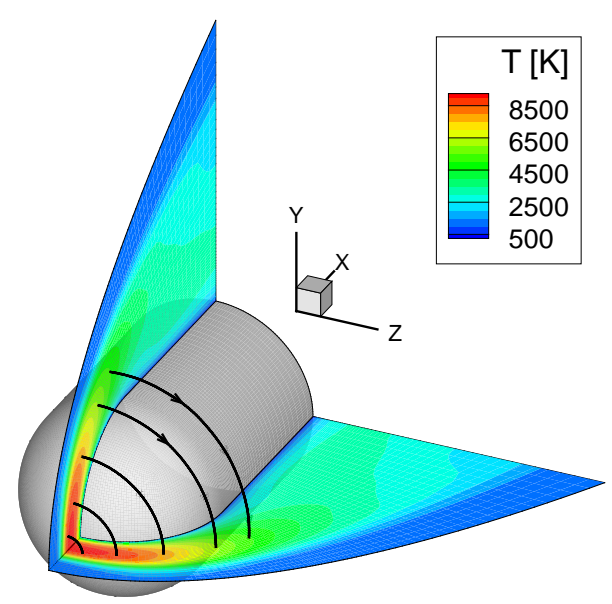

(b) Computing $\mathbf{E}$.

Figure 6.12: Temperature contours and current lines for Mach 4.75 argon flow around a hemisphere capped geometry with a $0.28 \mathrm{~T}$ magnetic field. Chapman and Cowling conductivity model) 
When employed, the MHD module iterates until it achieves a minimum convergence criterion or exceeds a maximum number of iterations (3000 iterations is specified for this scenario, but other scenarios typically require $10^{5}$ iterations). Figure 6.13 plots the L2 residual error from the current continuity equation in the MHD module for a portion of the flow simulation.

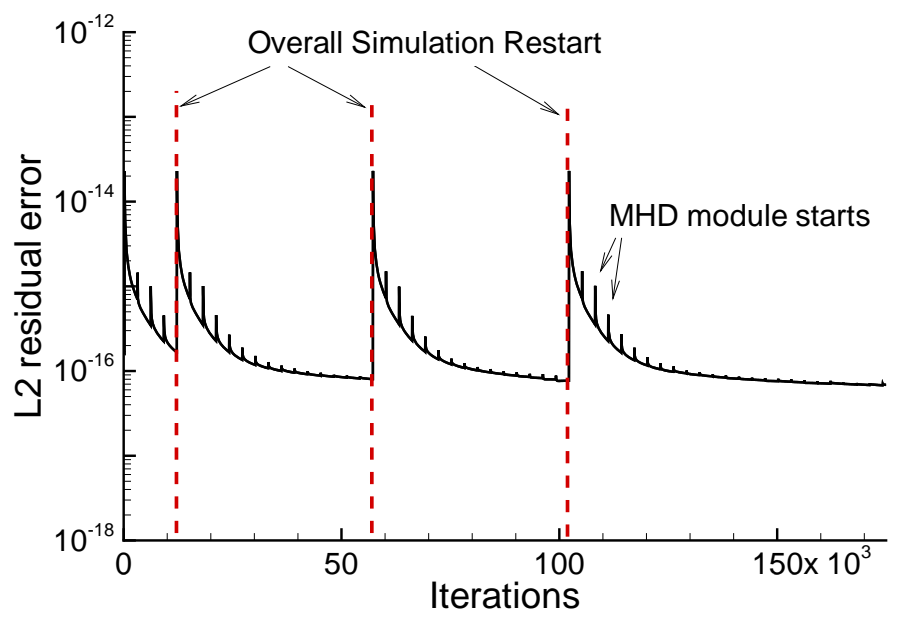

Figure 6.13: L2 residual error from the MHD module of a simulation of Mach 4.75 argon flow around a hemisphere capped geometry with a $0.28 \mathrm{~T}$ magnet. (Chapman and Cowling conductivity model)

The small fluctuations seen in the curve indicate where the MHD module exceeds 3000 iterations and is restarted 5 fluid iterations later. The larger separations (indicated as red dashed lines), indicate where the LeMANS-MHD code is restarted because the simulation exceeded the wall time allowed on the parallel computing cluster. Note that the electric potential, $\phi$, is not saved in the restart file, so it must be recomputed during simulation restarts.

Kranc et al. reported an increase in shock standoff distance due to the applied magnetic field [16]. The increase was measured by comparing photographs of the flow with and without the applied magnetic field. In their analysis, they assume the upstream edge of the shock can be inferred from the boundary of the flow's 
luminosity. This photographic technique for measuring the shock standoff distance was previously used by Ziemer [14] and Bailey and Sims [139] in similar experiments.

Although the computational solutions provide many ways of estimating the shock location, the change in shock standoff distance is computed by comparing the location, along the stagnation line, where the density ratio exceeds the ideal gas, infinite Mach number threshold for a normal shock wave:

$$
\lim _{\mathrm{M}_{1} \rightarrow \infty} \frac{\rho_{2}}{\rho_{1}}=\lim _{\mathrm{M}_{1} \rightarrow \infty} \frac{(\gamma+1) \mathrm{M}_{1}^{2}}{(\gamma-1) \mathrm{M}_{1}^{2}+2}=\frac{\gamma+1}{\gamma-1}
$$

where $M_{1}$ is the upstream Mach number, $\gamma$ is the ratio of specific heats, $\rho_{1}$ is the upstream density, and $\rho_{2}$ is the downstream density. Using this equation, the density ratio limit for argon is 4 ( $\gamma=5 / 3$ for argon). This approach provides a consistent method for defining the shock location, so it should provide adequate estimates of the change in shock standoff distance due to an applied magnetic field. Figure 6.14 plots the density ratio contours for several electrical conductivity models with a peak magnetic field of $0.13 \mathrm{~T}\left(1\right.$ telsa $=10^{4}$ gauss $\left.[\mathrm{G}]\right)$.

The change in shock standoff distance is computed for the various models, and plotted in Fig. 6.15 with experimental measurements collected using the photographic technique described previously.

The experimental uncertainty was $\pm 10 \%$ (error in determining shock location for one run), but the repeatability (difference in shock location between nominally identical runs) was $\pm 30 \%$ as seen for $\mathrm{B}_{\max }^{2} \simeq 13 \times 10^{6} \mathrm{G}^{2}$. Both the $2^{\text {nd }}$ order PRS model and Chapman and Cowling models match the experimental data well, with the $2^{\text {nd }}$ order PRS model fitting much better, particularly at larger magnetic field strengths. Solutions obtained by using Raizer's model observed almost no change in shock standoff distance because the model predicts a lower range of electrical 


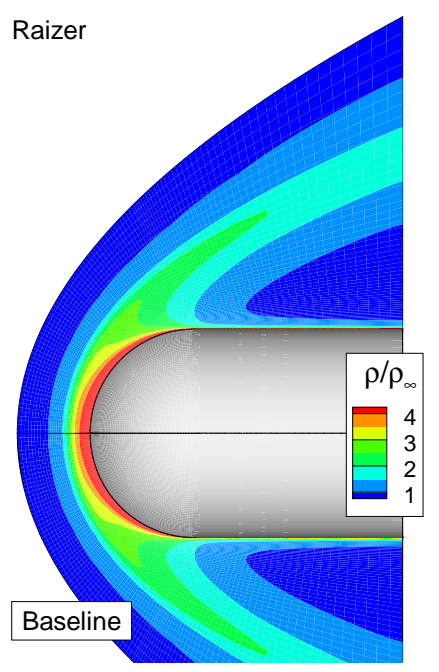

(a) Raizer

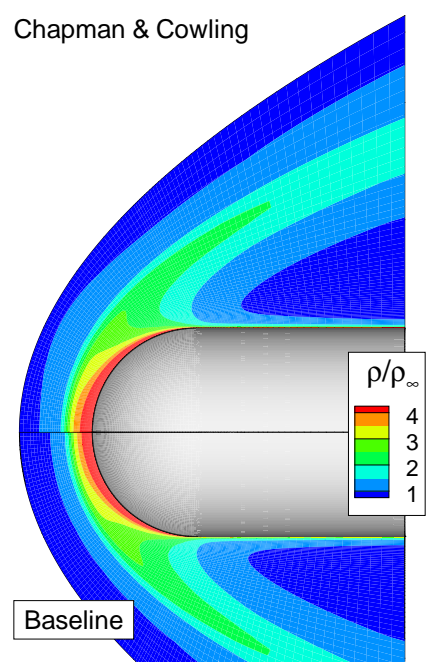

(b) Chapman and Cowling

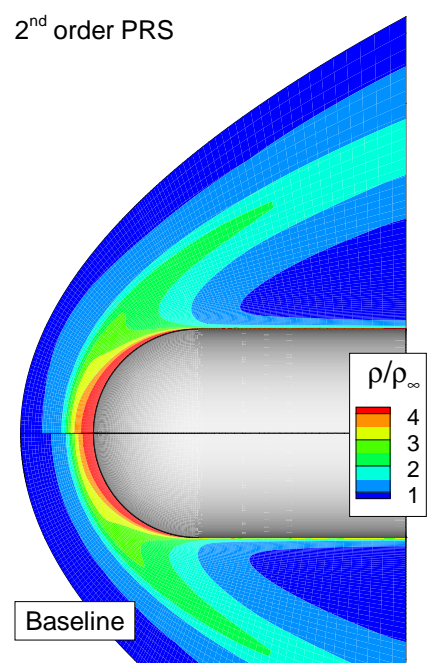

(c) $2^{\text {nd }}$ order PRS

Figure 6.14: Density ratio contours for Mach 4.75 argon flow around a hemisphere capped geometry for various electrical conductivity models. $\left(\mathrm{B}_{\max }=\right.$ $0.13 \mathrm{~T}$ )

condicitivities (as seen in Table 6.3). In addition, Raizer's model is only temperature dependant, so its peak conductivity is just downstream of the bow shock (where the temperature is the highest), yet the magnetic field strength has already significantly decayed due to its $\mathrm{r}^{-3}$ dependency.

The heat transfer to the surface for the various electrical conductivity models is shown in Fig. 6.16. Integrating the heat flux over the surface produces the total heating to the geometry. The change in peak heating is computing by comparing the heat flux at the stagnation point $\left(\Delta \mathrm{q}_{\mathrm{w}}=\frac{\mathrm{q}_{\mathrm{w}, \mathrm{MHD}}-\mathrm{q}_{\mathrm{w}, \text { baseline }}}{\mathrm{q}_{\mathrm{w}, \text { baseline }}}\right)$. Table 6.4 lists the percent change in peak heat flux and total heating for various magnetic field strengths and electrical conductivity models.

Except for the results from Raizer's model, the total heating to the surface actually slightly increases because of increased heating to the cylindrical portion of the geometry (i.e. aft of the stagnation region), due to the direction of the magnetic field lines in the region where the hemisphere capped forebody merges with the cylindri- 


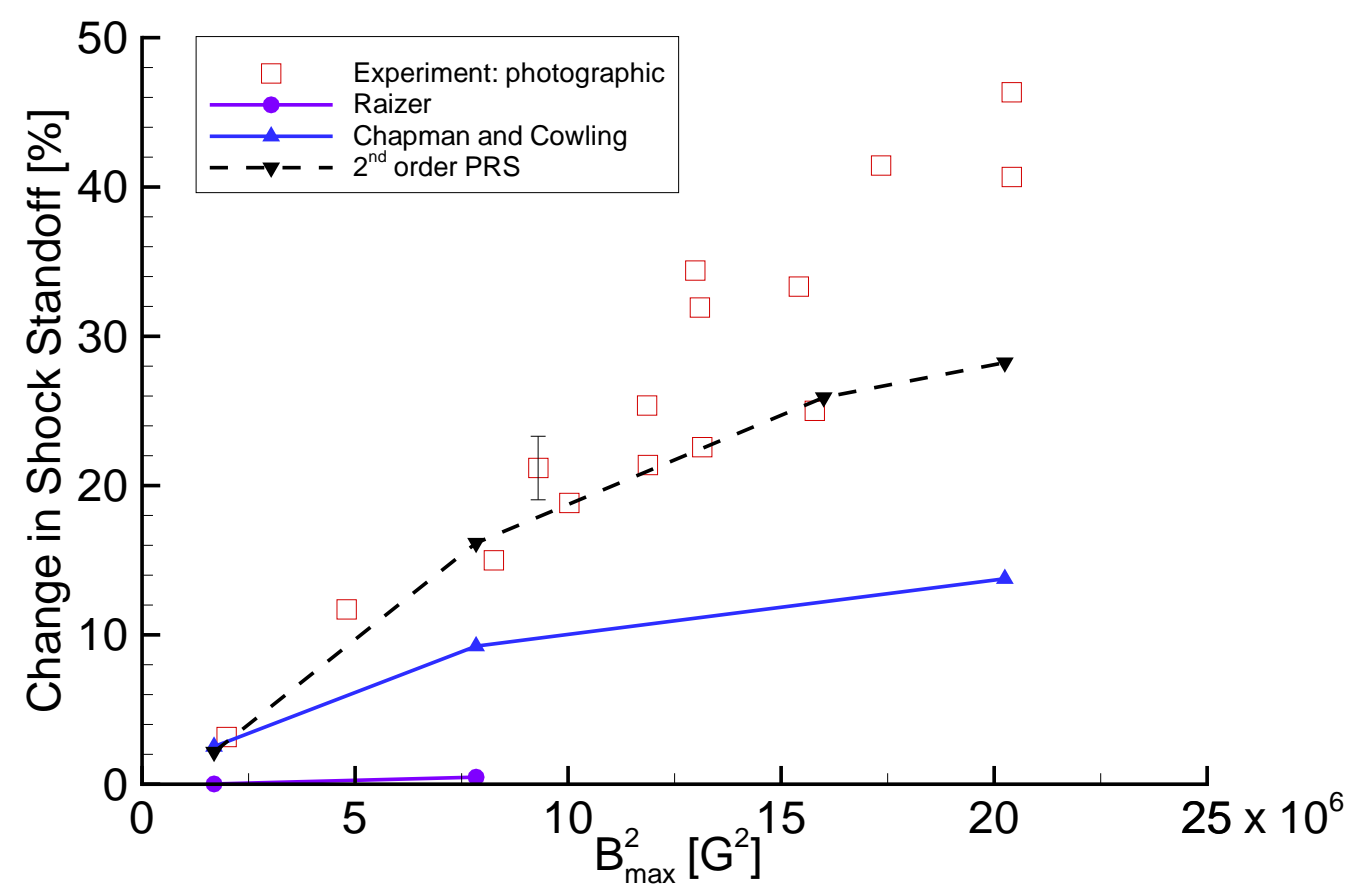

Figure 6.15: Percent change in shock standoff distance versus magnetic field strength for Mach 4.75 argon flow around a hemisphere capped geometry with various electrical conductivity models. Measurements from [16]. (experimental uncertainty $\pm 10 \%$ )

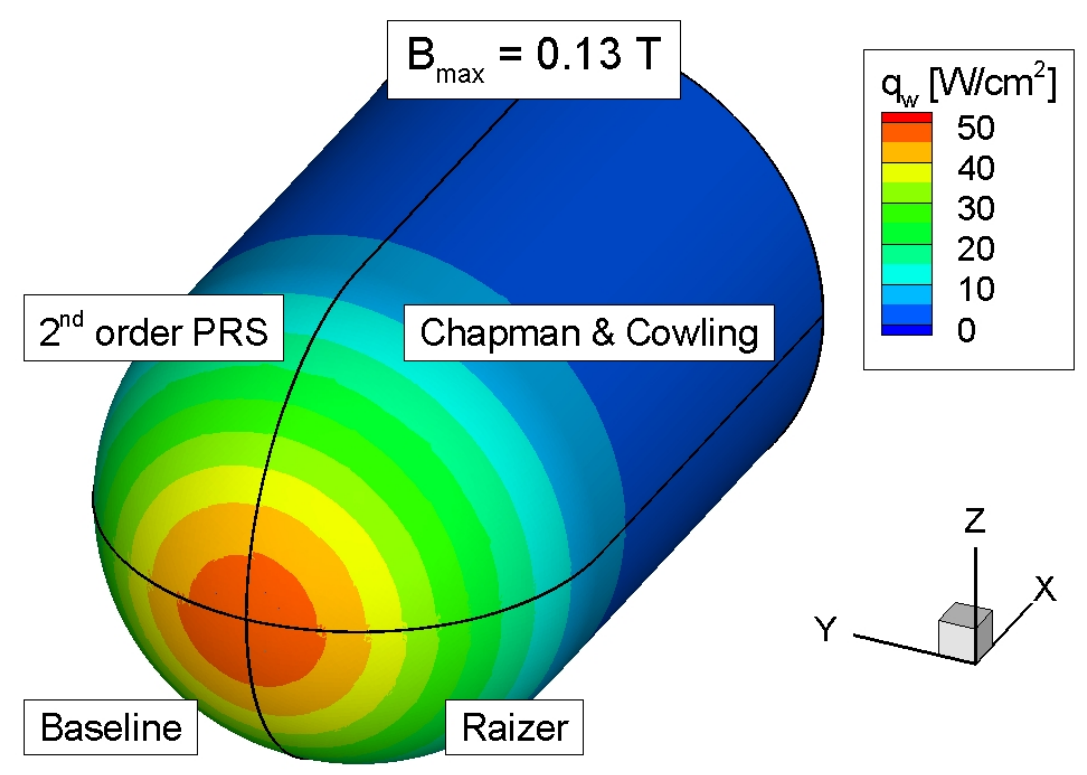

Figure 6.16: Heat flux contours for Mach 4.75 argon flow around a hemisphere capped geometry with a $0.13 \mathrm{~T}$ magnetic field and various electrical conductivity models. 
Table 6.4: Percent change in heat flux to the surface for Mach 4.75 argon flow around a hemisphere capped geometry with an MHD-Heat Shield.

\begin{tabular}{c|cc|cc}
\hline \hline & \multicolumn{2}{|c}{$\Delta$ Total Heating } & \multicolumn{2}{c}{$\Delta$ Peak Heating } \\
Model & $\mathrm{B}=0.13 \mathrm{~T}$ & $\mathrm{~B}=0.28 \mathrm{~T}$ & $\mathrm{~B}=0.13 \mathrm{~T}$ & $\mathrm{~B}=0.28 \mathrm{~T}$ \\
\hline Raizer & $-0.1 \%$ & $-0.3 \%$ & $-0.3 \%$ & $-1.2 \%$ \\
Chapman and Cowling & $0.6 \%$ & $1.5 \%$ & $-1.6 \%$ & $-6.3 \%$ \\
$2^{\text {nd }}$ order PRS & $0.4 \%$ & $1.1 \%$ & $-2.5 \%$ & $-5.6 \%$ \\
\hline \hline
\end{tabular}

cal aft section. Raizer's model fails to capture this behavior because its predicted electrical conductivity is too low throughout the domain, but particularly in the aft region where the freestream temperature is much cooler than in the bow shock. In general, an applied magnetic field moderately increases the total heating to the geometry, but significantly decreases the peak heat flux at the stagnation point. Both the $2^{\text {nd }}$ order PRS model and the Chapman and Cowling model yield similar results, but since the $2^{\text {nd }}$ order PRS model provides better estimates in the percent change in shock standoff distance, its results for the heat flux to the geometry may be more accurate.

\subsection{Conclusions}

In this chapter, two different plasma-based flow control devices were explored. Arc discharge simulations were carried out for Mach 12.6 air flow over a blunt leadingedge geometry, and thermal energy deposition was quantified as a function of the input voltage. These simulations help to assess the feasibility of the plasma-based flight control technique explored in Chapter III, using a phenomenological energy deposition model. An applied voltage of the order of kilovolts is seen to produce the heating rates on the order of kilowatts that were identified as necessary in Chapter 
III.

An MHD-Heat Shield concept was then explored for Mach 4.75 argon flow over a hemisphere-shaped forebody attached to a cylinder, corresponding to the experiment of Kranc et al.. The magnetic field worked to oppose and slow the flow near the stagnation region, and increased the shock standoff distance. The increase in shock standoff distance decreased the peak heating to the body (at the stagnation point), but also increased the total heating to the geometry because of increased heating to the cylindrical portion of the body. This result has important implications for design of MHD-Heat Shield devices: they can reduce peak heat flux, but may incur a penalty in total heating. Since both peak and total heat load are important aspects to consider when designing a thermal protection system, this technology provides additional scenarios for vehicle designers to evaluate.

The scenarios examined clearly showed that the effectiveness of the plasma-based MHD technologies explored in the chapter are highly dependent on the electrical conductivity present in the flow. Since the flow conditions for the MHD experiment lay outside the bounds used to calibrate the PRS models, the Chapman and Cowling conductivity model produced the best results for this flow. Predictions based on this model for changes in shock standoff were within about $30 \%$ of experimental values, and could be improved by calibration of the conductivity model. 


\section{CHAPTER VII}

\section{Conclusions}

The main objective of this dissertation was to explore the feasibility of flight control and thermal shielding for hypersonic vehicles using methods based on electromagnetic flow control. Computational tools were developed to model these technologies, and used to bound their performance requirements. While the technologies were seen to be physically plausible, their actual viability in hypersonic vehicles will strongly depend on the efficiency of their implementation, particularly in terms of weight and power consumption, which are two principal factors that drive aircraft design. The following sections provide a brief summary of the entire thesis, a detailed review of the major contributions, a summary of improvements made to the flow solver, and concludes with an outline of recommendations for future research.

\section{$7.1 \quad$ Summary}

Having the research tools available to investigate electromagnetic flow control in hypersonic flows is particularly valuable at present, since the area has experienced a resurgence of research interest. The revival has been credited to many factors, including the expanding requirements for sustained hypersonic flight and rapid access to space. In addition, the numerous mechanical and material advances made during 
the past half-century have given the area of flight-weight magnetoaerodynamics the rejuvenation necessary to warrant further exploration. As such, research in the area has focused on using plasma-based flow control devices to either enhance or replace existing (usually mechanical), systems. Experimentally, these devices show promise, but require additional research and refinement before they are widely accessible and acceptable as realistic alternatives to traditional methods of flow control. Unfortunately, experiments in this area are extremely expensive and very limited due to the extreme conditions typically present in the flight regimes where the applicability of the plasma-based devices are being investigated. Therefore, much of the research has focused on developing and using computational tools capable of accurately modeling these technologies.

Chapter II outlined the flow solver, LeMANS, which is used throughout this thesis. LeMANS is an unstructured finite-volume CFD code that solves the NavierStokes equations for thermal nonequilibrium, chemically reacting, hypersonic flows. In particular, the thermal nonequilibrium and chemically reacting features make the solver preferable for exploring plasma-based flow control devices in hypersonic flows, because the high kinetic energy dissociates and ionizes the gas as it passes through the strong bow shock produced by the vehicle. Accurately accounting for the chemical reactions is crucial for predicting the flow conditions in, and around, the plasma-based flow control devices being investigated. In addition, these devices usually modify the flow through force and/or energy exchange, which can change the species composition and other flow characteristics downstream of the device. These changes may include differences in pressure and heat transfer at the vehicle surface, properties traditionally important to design and safety of a hypersonic vehicle and its payload. 
The thermodynamic relaxation rates of different energy modes can vary significantly in some gases (e.g., air), which can lead to unique flow conditions and should be modeled accordingly to improve the accuracy of the simulations being conducted. As previously mentioned, the plasma-based devices being investigated modify the flow through force and/or energy exchange, however, some are capable of only performing these exchanges with specific gas species or energy modes (e.g., a tuned microwave emitter may only excite the vibrational states of particular molecules). Including thermodynamic nonequilibrium allows the device's effects to be more accurately modeled.

Chapter II also discussed results from validation exercises of the flow solver which was used to simulate sharp-and blunt-nose elliptic cone geometries at hypersonic speeds, both of which were compared to existing experimental data, and found to be in excellent agreement. The chapter concluded with a summary of the new features available in the solver.

Although other studies have focused on developing and using accurate computational tools to explore the effects of various plasma-based devices, an open question remained as to whether the required power expenditures for such devices can be achieved for practical systems. Chapter III addressed this question by presenting results from a numerical study for hypersonic flow over a blunt-nose elliptic cone geometry with a thermal actuator. The thermal actuator deposited energy into the flow and was represented as a phenomenological dissipative heating model. The investigation revealed that the shape of the deposition had minimal impact on the effectiveness of the deposition, which suggests that the selection of a plasma-based flow control device for energy deposition should be largely influenced by how much energy it can deposit into the flow, rather than the distribution of the deposition. 
Hot wall temperatures were found to negatively impact the effectiveness of the deposition since the local total energy is higher than for the cold wall simulations, so less energy can be deposited into the translational energy mode. Rather, a larger potion of the deposited energy is stored in internal energy modes (which do not affect the local pressure or flow control). The investigation was conducted for several vehicle configurations and flight regimes, and it found that the effectiveness of volumetric energy deposition for flight control scales strongly with a nondimensional parameter based on the freestream flow kinetic energy flux. This nondimensional scaling favors configurations at higher altitude, with slower velocities, and smaller vehicle length.

As described in Chapter IV, a magnetohydrodynamics module was developed, and coupled to the flow solver, to model the electromagnetic effects in the flow. This module replaced the simplified volumetric heating model, described in Chapter III, with a more physically accurate model that accounts for both force and energy exchange due to the presence of electromagnetic fields. The MHD module was developed for flows that have a low magnetic Reynolds number, so only externally applied magnetic fields are present (and must be specified). A generalized form of Ohm's law, which accounts for the Hall effect, is solved to determine the electric and current density fields, all of which constitute the electromagnetic effects present in the flow. The MHD module is parallelized using the same routines as the flow solver, and it accommodates three-dimensional grids. Validation of the module was accomplished by computing flows between infinite, segmented plate electrodes.

One of the main parameters required to solve Ohm's law in the MHD module is the electrical conductivity. The electrical conductivity is a transport property that is usually determined using semi-empirical models because of the prohibitive computational cost associated with determining it directly by solving Boltzmann's 
equation. Chapter V explored several existing semi-empirical models to illustrate their strengths and deficiencies. The chapter also included direct solutions of Boltzmann's equation, and good agreement was found with the semi-empirical models over their range of validity. These Boltzmann solutions clearly demonstrated, however, where the existing models break down. Although the use of the semi-empirical models of the electrical conductivity is acceptable in some cases, it is not general, and is problematic when investigating scenerios outside the bounds of the models.

Chapter $\mathrm{V}$ went on to outline a procedure to develop a suitable surrogate model to solutions of Boltzmann's equation. This procedure provides a general approach to developing an electrical conductivity model, which is valid across an entire design space, and is suitable for any species composition. Details of improving the accuracy and efficiency of the surrogate model by conducting global sensitivity analysis and by using reduced order modeling were also discussed. Third-order PRS models for weakly-ionized argon and air were developed, and found to be sufficiently accurate across the entire design space. This means most of the accuracy and generality of the Boltzmann solver is retained without the computational cost associated with directly coupling the Boltzmann solver to the MHD module.

The chapter concluded with a scenario where the semi-empirical and surrogate models of electrical conductivity were applied to a three-dimensional blunt-nose elliptic geometry which employed an MHD-Heat Shield. While the range of electrical conductivities predicted by the models was fairly consistent, the electrical conductivity contours varied significantly, which led to significant differences in the effectiveness of the MHD-Heat Shield, and further illustrated the importance of using an appropriate electrical conductivity model when accounting for electromagnetic effects. 
In Chapter VI, the components developed in previous chapters were brought together to investigate two different types of plasma-based flow control devices. A glow discharge over a three-dimensional blunt leading edge was simulated to show energy deposition as a function of imposed electrical potential, and to further illustrate the importance of the electrical conductivity. Similarly, the MHD-Heat Shield concept was further explored. In this scenario, a three-dimensional, hemisphere-shaped geometry was used to investigate the influence the various electrical conductivity models, the Hall effect, and magnetic field strength had on the usefulness of employing the MHD device as an effective means of heat transfer mitigation.

\subsection{Contributions}

The work represented by this thesis is significant because it provides several computational tools, previously unavailable to the community, that allow the investigation of electromagnetic effects due to plasma-based flow control devices in hypersonic flows. Further, these tools were used to demonstrate the physical plausibility of plasma-based flight control and thermal shielding, and to bound the performance requirements of prospective systems.

\subsubsection{Major Contributions}

- This dissertation established the power required for flight control based on volumetric heating for a typical reentry condition as $\sim 1 \mathrm{~kW}$ deposited into the flow. This is on the same order of magnitude as the auxiliary power systems on reentry flight test vehicles under development [140]. Thus, this concept is right on the edge of being feasible, and its success strongly depends on efficiency of implementation. These results motivate detailed research into efficient implementation of such systems. 
- This dissertation presented a nondimensional scaling for pitching moment versus power deposition. The scaling turns out to favor smaller vehicles, which may make a plasma-guided artillery shell a high-payoff application $[141,3]$. This scaling may also provide a guide for future research on plasma-based flight control.

- This dissertation demonstrated that commonly-accepted electrical conductivity models have serious deficiencies, especially when used outside their recommended range of validity. These limitations have been removed in this dissertation by developing a computationally-efficient and accurate alternative model, a surrogate model to solutions of Boltzmann's equation. In addition, the generalized, detailed procedure presented in this thesis allows for the development of new electrical conductivity models for any gas composition.

- This dissertation examined the MHD-Heat Shield concept and found an applied magnetic field increased the shock standoff distance, which decreased peak heating to the body (at the stagnation point), but also increased the total heating to the body because of increased heating to the cylindrical portion of the body.

- In order to achieve the main goals of this dissertation, a MHD code suitable for modeling hypersonic vehicles was developed. This code's key features include: a realistic conductivity model, realistic thermal and chemical nonequilibrium, and ability to compute computing complex geometries (i.e., three-dimensional, unstructured, and parallel capabilities). This code will prove to be substantially more useful than codes previously employed in this field, all of which lack at least some of these features. 


\subsubsection{Improvements to LeMANS}

- Three-dimensional Validation: Although the flow solver has been previously validated by other researchers, the cases presented in Chapter II clearly demonstrate it is capable of simulating three-dimensional, hypersonic, laminar, chemically-reacting, thermodynamic nonequilibrium flows. It is within this flight regime where plasma-based flow control devices appear to be viable and were investigated throughout this thesis.

- Phenomenological Heating Module: The flow solver now contains a module capable of directly depositing energy into specific locations within the domain. This feature is useful for investigating the downstream effects that a heater, or any energy deposition device, has on the flow-field, and was used as such in the investigation conducted in Chapter III.

- Magnetohydrodynamics Module: A three-dimensional, parallelized, magnetohydrodynamics module, capable of accommodating nonuniform structured grids, is coupled to the flow solver. This module provides physically accurate modeling of electromagnetic effects, accounts for the Hall effect, and is not detrimental to the overall parallel efficiency of the code, as detailed in Chapter IV. This module was essential to investigating the arc discharge and MHDHeat Shield concepts explored in Chapter VI.

- Electrical Conductivity Module: All the semi-empirical electrical conductivity models detailed in Chapter $\mathrm{V}$ are accessible to the MHD module, although they were shown to have deficiencies when used in certain flight regimes. The MHD module is also capable of accessing PRS surrogate models of solutions to Boltzmann's equation (up to $6^{\text {th }}$ order), which provides more accurate 
estimates of the electrical conductivity at slightly higher computational expense.

\section{- Procedure for Generating Accurate Electrical Conductivity Models:}

A general procedure is outlined for developing a suitable surrogate model from solutions to Boltzmann's equation using [121], which is a surrogate modeling tool suite developed as a Matlab library. This includes additional routines that allow the development of an electrical conductivity model for any species composition.

- PRS Models for Argon and Air: The generalized procedure for developing a surrogate model was used to develop accurate surrogate models for weaklyionized argon (3 species), and air (11 species), from solutions of Boltzmann's equation. Both models provide the accuracy and generality of the Boltzmann solver without significantly increased computational expense, and were vital to investigating the arc discharge and MHD-Heat Shield concepts explored in Chapter VI.

- Pre-LeMANS: This program partitions the grid based on the number of processors to be used in the simulation. The routine is vital when simulating flows using large grids because the partitioning subroutine in the flow solver has a grid size limit. This program was required to simulate the flow-field around the realistic blunt-nose elliptic cone geometry used in Chapters II, III, and V, because of the large three-dimensional grid required to obtain grid-independent results.

- Post-LeMANS: This program runs after the parallel flow solver has obtained a solution. Duplicate information contained in the various solution files is 
removed, and the remaining information is combined into a single solution file, which facilitates the use of existing visualization programs. This program was used to combine the solution files created for all the simulations presented in this thesis.

\subsection{Future Work}

While this thesis has shown that it is physically plausible to using electromagnetic flow control for flight control and/or thermal shielding in hypersonic vehicles, the viability of doing so strongly depends on the efficiency of the implementation of the technologies. As such, continued research in this field is warranted to further understand, model, and explore the electromagnetic effects in hypersonic flows. The following is a list of recommendations for future research.

1. Improve the MHD routine to allow two-dimensional and axisymmetric grids. While real hypersonic flows and their accompanying electromagnetic properties are three dimensional, there are instances when two-dimensional or axisymmetric approximations are sufficient. Reducing the spatial dimensions of the fluid and MHD solver would significantly reduce the computational effort required to produce a solution. Fortunately, the methodology outlined in Chapter IV is suitable for two-dimensional domains.

2. Incorporate the ion-slip phenomenon into the electrical conductivity tensor. Since the electrical conductivity is already expressed in tensor form, the addition of ion-slip effects to the code is trivial. Validation could be accomplished by performing scenarios outlined by Oliver and Mitchner for flow between two segmented electrodes [98], which were used in Chapter IV to validate the im- 
plementation of the Hall effect. Accounting for this effect is necessary when there is momentum uncoupling between the ions and neutral particles.

3. Modify the finite-volume method used in the MHD routine to allow for unstructured grids. This would allow the MHD routine to be consistent with the flow solver, which is capable of using unstructured grids. A fairly significant effort would be needed to develop and implement a suitable finite difference scheme that is consistent with the flow solver, but it may be possible to use some of the existing features in the flow solver to accommodate unstructured grids. Having this capability would decrease the time spent generating a uniform grid with sufficient resolution in regions of complex flow structure and electromagnetic phenomena (which may exist in different locations of the domain).

4. Modify the species mass diffusion flux, $J_{s}$, for ionized species to account for electromagnetic effects. This influence is currently neglected in the ionized species diffusion flux calculations and should be included for improved physical accuracy.

5. Incorporate automatic grid refinement in both the flow solver and the MHD routine. This feature would decrease time spent generating a grid, which usually requires at least one preliminary grid to determine the important flow features (i.e., the shock location, boundary layer thickness, etc.).

6. Add a turbulence model to the flow solver. Although the flows investigated in this dissertation have a Reynolds number which is small enough to justify the greatly simplifying assumption of laminar flow, it would be beneficial to incorporate a turbulence model. This feature may be necessary as the fluid-MHD code is used in flight regimes and configurations where the laminar assumption 
may not be valid (e.g., plasma-based flow control for RAM engines, plasmaguided artillery shells fired from rail guns).

7. Allow for additional surrogate models in the electrical conductivity module. Currently, the module provides the user with several semi-empirical options and a PRS surrogate model. While the PRS model developed in this dissertation provides sufficient accuracy and computational cost similar to the semi-empirical models, other surrogate approaches (e.g., Kriging, radial-based neural networks, support vector regression, etc.), may offer better performing options. A detailed study of other surrogate models available, and their applicability, should be conducted to determine it they are more suitable and whether the additional cost (both in computational time and memory), is worthwhile. 


\section{APPENDICES}




\section{APPENDIX A}

\section{Pre-LeMANS}

The CFD code, LeMANS, utilizes METIS [68] to partition the domain (mesh) amongst multiple processes. METIS is a serial routine, so each processor must load and process the entire mesh. A serial approach is also necessary when employing the line implicit iterative routine to advance the solution toward convergence, because the 'line finder' subroutine in LeMANS also requires the entire mesh to appropriately group cells, which improves the layout of the sparse linear system of equations, as described in [67]. Once finished, the root processor partitions its mesh into NP parts (where $N P$ is the total number of processors). This information is sent to the other processors, that remove their 'unwanted' parts of the original mesh, thereby significantly reducing the processor RAM requirement. Each processor also determines its partition's face connectivity information, which is essential for the MPI calls used within the parallel version of LeMANS. The entire process is illustrated in Fig. A.1. Although this approach works, it is problematic for large simulations, because each processor must be capable of storing the entire mesh, and the root processor needs significantly more RAM to partition its mesh.

Although the partitioning process itself is highly efficient, the overall methodology is computationally wasteful and memory intensive. In fact, the memory requirements 


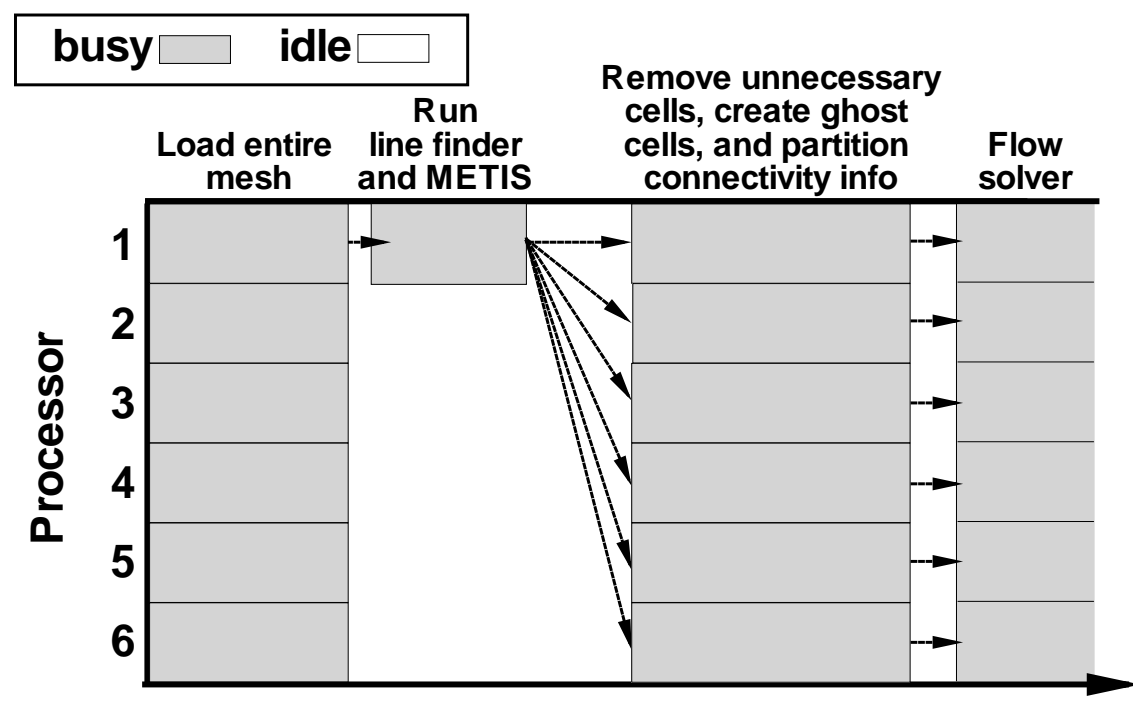

Total Time

Figure A.1: Diagram of the methodology for partitioning the mesh within LeMANS.

limit the domain size to less than 2.5 million cells when the processors have 2 GB of RAM. While this grid size is sufficiently large for many cases of interest, it is still beneficial to remove this limitation. In addition, the solver is typically employed to investigate a parametric study of several variables using the same mesh, therefore it is computationally more efficient to only partition the domain once.

Pre-LeMANS removes the maximum cell restriction by moving the initial loading and partitioning sequences out of LeMANS and executing them in a serial program which outputs each processor's necessary information as a separate 'partitioned mesh' file. In addition, the flow solver is modified to accommodate the 'partitioned' files, while ensuring the necessary connectivity information is not lost.

\section{A.1 Methodology}

The partitioning procedure outlined in LeMANS is computationally wasteful as a parallel routine, however, it is appropriate for a single processor procedure. Therefore, Pre-LeMANS is based on the method outlined in Fig. A.1, except it is written 
serially. However, since only one processor exists, parts of the routine are repeated for each mesh partition, as seen in Fig. A.2.

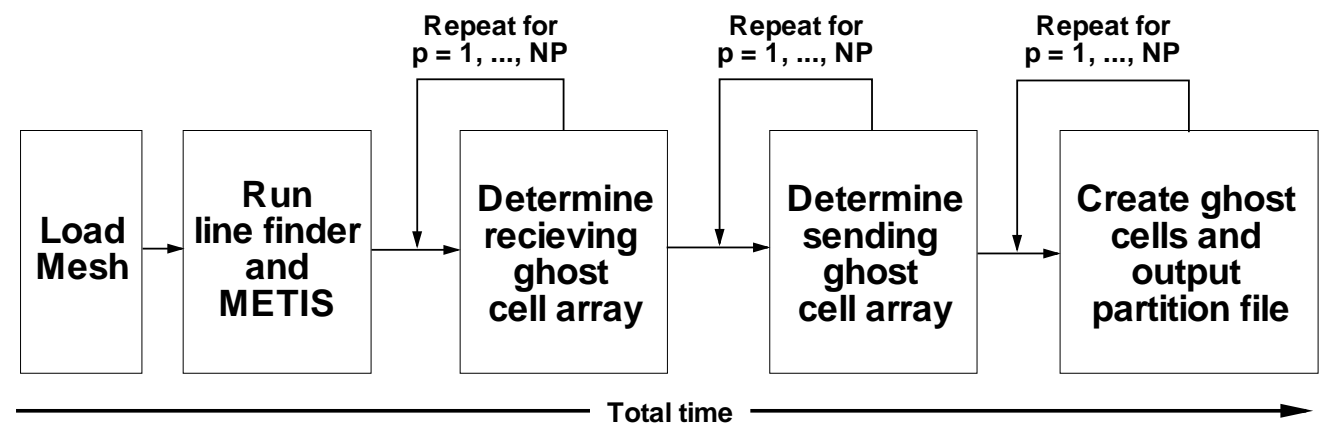

Figure A.2: Cartoon of the methodology for partitioning the mesh in the PreLeMANS code.

Although Pre-LeMANS still requires a single processor with enough memory to store the entire mesh and partition it, this procedure is now able to be run on a larger variety of systems, including large memory or shared memory systems with only one or a few processors. The solver can still be run on a parallel computing platform with limited memory per processor.

\section{A.2 Modified LeMANS}

An if/else statement is added to the main routine of the flow solver (lemans.c), and, depending on the input file, will either partition the domain using the original procedure, or load the partition files. If the partition files are not present in the directory, the flow solver displays a warning, then executes the Pre-LeMANS routine using the root processor. LeMANS can also be run in its original mode, where partitioning procedures exist as described in Fig. A.1.

If the partition files are present, they are loaded into their respective processors. Although Pre-LeMANS determines the appropriate breakdown of the domain and connectivity information, it does not provide all the cell specific information nec- 
essary for the solver to work correctly. This decision is made to minimize the size of the partition files. Therefore, once the partition files are loaded, each processor determines its cells' characteristic size and grid face metric information. These subroutines (cell size and face information), are already present in the original version of LeMANS and are easily computed based on the information provided by the 'partition' files.

Pre-LeMANS is a preconditioning routine which may be necessary when computing flow-fields that require large meshes (greater than 1 million cells), as the solver may not be able to partition the mesh using its internal procedure due to memory requirements. In addition, using this routine improves the parallel efficiency of the solver since all its processors can immediately start solving the conservation equations (rather than sitting idle while the root processor determines the partitions). Finally, employing Pre-LeMANS reduces the computational time when conducting a parametric study of parameters that require the same mesh and number of processors. 


\section{APPENDIX B}

\section{Post-LeMANS}

Parallelization of the solver, LeMANS, which is used throughout this thesis, allows previously computationally prohibitive scenarios to be investigated. The parallel version of LeMANS generates an output file for each processor used in the simulation. Each file contains that processor's cells, accompanying nodal values, and face connectivity information. Some of these cells are duplicated between various output files. These duplicate cells create 'interior walls' within the visualization software that make it challenging to visually analyze the flow-field, and are a nuisance when trying to create translucent three-dimensional flow-field contours for presentations and publications.

Post-LeMANS erases these 'interior walls' by removing the duplicate data within the output files, updating the cell connectivity information, and combining the resulting data into a single file. Of these tasks, the most important is the preservation of the cell face connectivity, since LeMANS is an unstructured Navier-Stokes solver. The routine is parallelized using MPI calls, not only to reduce the run time necessary to reorganize the data, but also to reduce the individual processor's memory requirements needed to store temporary data arrays. 


\section{B.1 Search Algorithms}

One of the most straight-forward approaches is to load each output file into a single matrix one row at a time (vertices $\times$ information). Each row contains all the information about one vertex (node) in the simulation. Before a new row is added, all the existing rows are searched to see if the new data was previously added by a different processor's output file. The comparison is accomplished by examining the $x, y$, and $z$ coordinate values of the node. If the node already exists in the matrix, the new node is skipped and the appropriate face connectivity information is updated. This method is illustrated in Fig. B.1.

\section{1) Compare new entry data to existing data in matrix.}

\section{2) If the new entry data match existing data, they are disgarded and their face connectivity file is updated accordingly.}

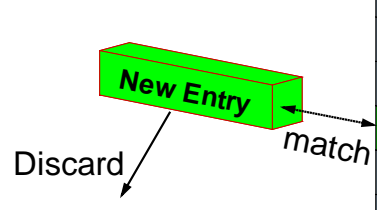

(b) Match does not exist.

(a) Scan for match.

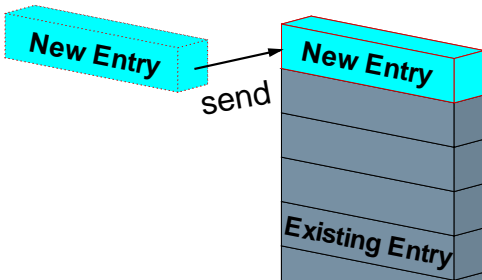

\section{3) If the new entry data do not match existing data, they are added to the existing data matrix and the process is repeated.}

(c) Match exists.

Figure B.1: Methodology of a systematic search in the Post-LeMANS code. 
This line-by-line or 'systematic search' is necessary because the previous entries are not ordered for this unstructured solver, so there is little benefit to be gained by sorting the existing matrix first. Likewise, putting the new entries in sorted order may not reduce the search time because each node is not equally spaced from its neighbors. In addition, sorting the entries greatly complicates the adjoined connectivity information as each connectivity profile would need to be updated for each new entry added, thus creating many additional computations.

The simplicity of the systematic search approach makes it easy to implement, but since the number of rows searched increases for every new entry, the approach becomes increasingly slow as the size and number of output files increases. This makes the 'systematic search' method ill-suited for merging multiple three-dimensional output files and is not used in the final version of the routine.

Realizing that the order in which the mesh is loaded into LeMANS is unimportant, and that the original output files are already self-contained (i.e., they have all the necessary information to work independently), a second approach is developed to improve the efficiency of the search algorithm for large data sets. In this approach, a three-dimensional cubic array, instead of a matrix, stores the data from the output files, where each slice of the cube corresponds to an output file as illustrated in Fig. B.2.

After uploading all the data from each output file into its respective slice of the cube, the data from the first slice are written to the new output file and, concurrently, its entries are compared to the data in each of remaining slices following the methodology outlined by the 'systematic search' in Fig. B.1. If a match exists in the subsequent slices, the entry is removed from the slice, but any connectivity information that was originally linked to the slice entry is updated to link to the entry 


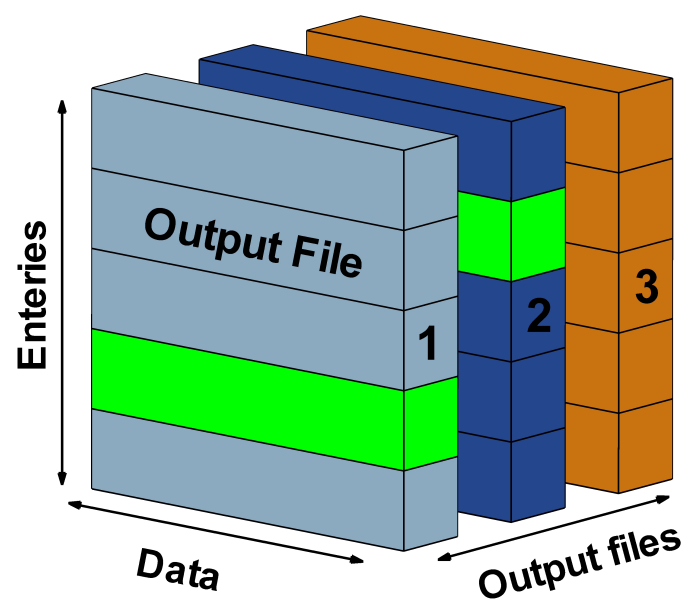

Figure B.2: Illustration of cube storage of the output files.

already located in the new, combined, output file. When each slice finishes writing to the combined output file, the total number of rows added to the new output file is also sent to the remaining output files to update their face connectivity information. The procedure is repeated for each remaining slice, as illustrated in Fig. B.3.

The 'cubic systematic search' method improves the efficiency of the search algorithm versus the original 'systematic search' described previously. For the systematic search, a total of: $\sum_{i=1}^{m k}(i-1)=1 / 2 m k(m k-1)$ searches are needed, where $m$ is the total number of files and $k$ is the total number of entries in a given file (assuming $k$ is the same for each of the $m$ files). By comparison, the 'cubic systematic search' only requires: $\sum_{i=1}^{m-1} k^{2}(m-i)=1 / 2 m k(m k-k)$ searches. While the cubic search does not provide a lot of improvement for small meshes, a typical million cell grid with twenty output files, requires approximately 25 billion fewer searches using the cubic systematic search routine. 


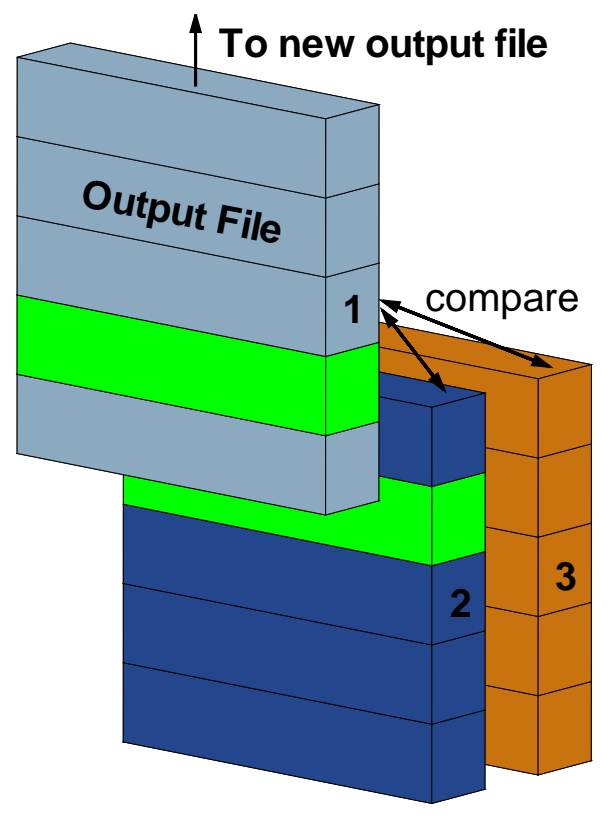

(a) Process $1^{\text {st }}$ output file

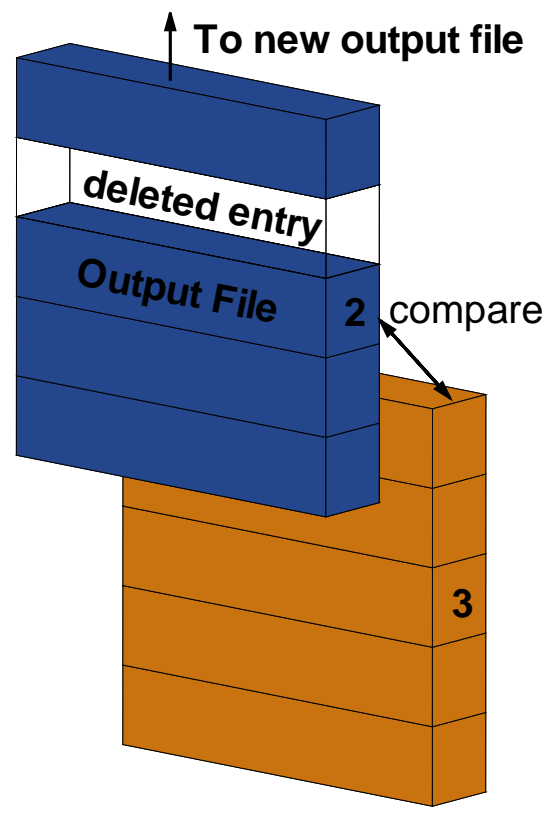

(b) Process $2^{\text {nd }}$ output file

Figure B.3: Cartoon explaining the cubic systematic search routine in the PostLeMANS code.

\section{B.2 Parallelization}

The main goal for parallelization of the routine is to maintain good efficiency while reducing program run time. Fortunately, the nature of the cubic systematic search makes it easy to extend the code into a parallel format. When used in parallel, each processor creates a cube to store its data. The size (depth) of the cube depends on how many output files exist and how many processors are being used. The files are divided sequentially amongst the processors, as seen in Fig. B.4 for 8 output files and 3 processors. A sequentially distribution is employed to ensure maximum load balancing as files are removed from the search.

As with the serial case, the first or root processor (corresponding to output file 1), writes file 1 to the new, combined, output file while searching its remaining slices (files 4 and 7), for duplicates. At the same time, the root processor sends a copy of the output file to each of the other processors, so they can search their slices for 


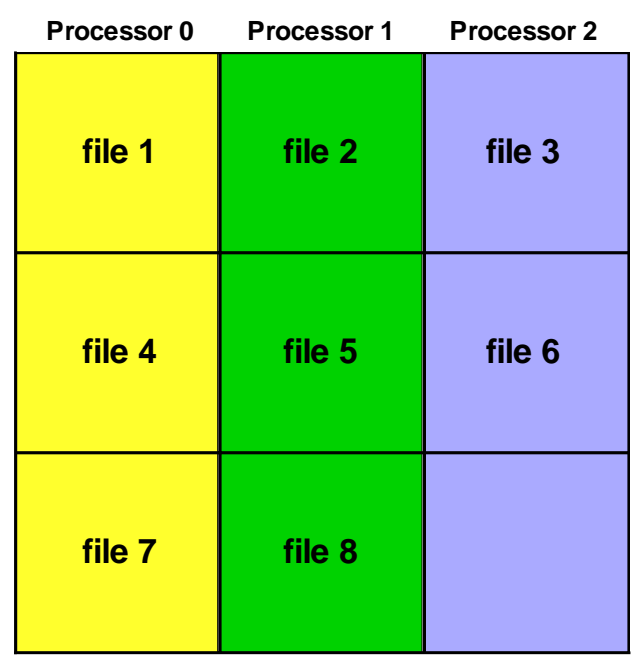

Figure B.4: Distribution of 8 output files to 3 processors for the Post-LeMANS code.

duplicates. Once finished, the next processor (corresponding to the output file 2), becomes the root processor and repeats the procedure until all the files have been written.

As the program proceeds through the slices in the matrices, the number of remaining entries that need to be searched decreases, which minimizes the need for dynamic load balancing since all processors will be busy until the last 'row' of output files needs to be processed (recall Fig. B.4). During this last row of searches, some processors sit idle, but their idle time is small compared to the overall computation time of the program, as illustrated in Fig. B.5. In addition, the increased communication cost to perform parallel line-by-line searches is greater than the computational cost of simply conducting the search locally. This is because the individual slices (output files) can not easily or efficiently be split due to their accompanying face connectivity information.

Although Post-LeMANS can run with any number of processors, $p$, the most processors it can actually use is equal to the number of output files, $m$, (i.e., $p \leq m$ ). 


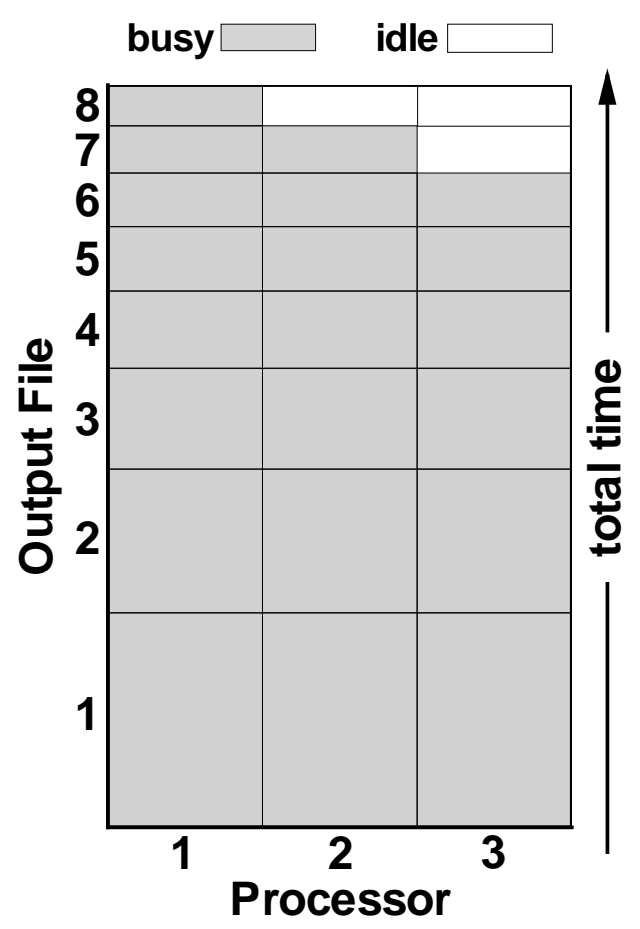

Figure B.5: Time and processor load for 8 output files and 3 processors for the PostLeMANS code.

MPI calls are used to transfer information between processors. Parallel efficiency is estimated by calculating the speedup, as seen in Eqn. B.1.

$$
\text { speed up }=\frac{\text { serial time }}{\text { parallel time }}
$$

where 'parallel time' is the wall time for the simulation (total CPU-seconds / number of processors). Speed up is measured by running the program to compile 8 output files, each containing about 20,000 cells. The program finishes quickly since the domain is relatively small (even for the serial case). In order to reduce scatter and improve confidence in the results, the program is run several times for each scenario of processors. Figure B.6 plots the 'speed up' versus the number of processors.

The figure shows that the efficiency of parallel Post-LeMANS is less than ideal. The 'speed up' plateaus as the number of processors approaches the number of files 


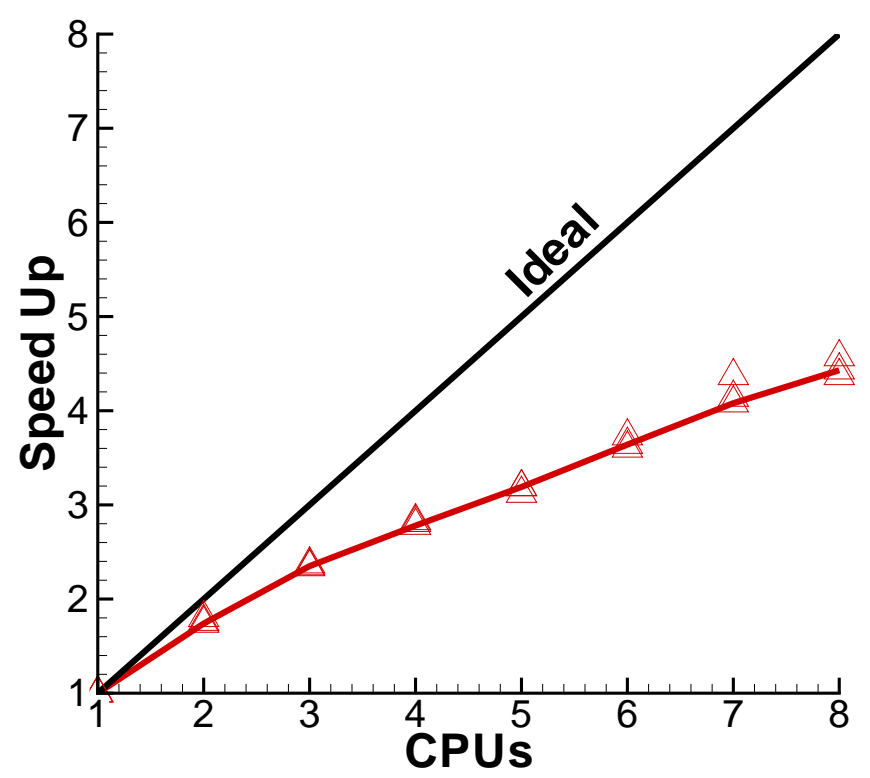

Figure B.6: Speed-up versus number of processors for the Post-LeMANS code with 8 output files.

available. This performance characteristic is not surprising given the methodology used to distribute work amongst the processors. As the number of processors increases, the number of processors that are idle during the 'last row' of searches also increases (recall Fig. B.5). In addition, the search time for each remaining output file decreases for each output file searched. If the size of the files grows (i.e., LeMANS uses a larger mesh on the same number of processors), then the parallel efficiency should improve because the run time for the initial output file searches would be much greater than the last few output file searches.

\section{B.3 Conclusions}

The main goal of the task described in this appendix was to develop a parallel code that could take the multiple files produced by the CFD code, LeMANS, and combine them into a single file while removing duplicate information. This was accomplished using MPI calls with special attention taken when implementing the code so the 
unstructured face connectivity information associated with the volumetric cells in the files is preserved appropriately. The resulting code is significantly faster than its original serial version (with systematic searches), and can use any number of processors, $p$, less than or equal to the number of output files, $m$, to be combined $(p \leq m)$ 


\section{APPENDIX C}

\section{Phenomenological Results}

The resultant pitching moments, $M_{p}=M_{p}$ (deposition) $-M_{p}$ (baseline), for the phenomenological blunt elliptic cone simulations with ellipsoidal energy deposition are listed in the following tables.

Table C.1: The pitching moment for the 'small' blunt elliptic cone simulations with freestream conditions listed in Table 2.2 and deposition shape parameters listed in Table 3.7. $\left(L=0.21 \mathrm{~m}, \mathrm{~T}_{w}=294 \mathrm{~K}\right)$

\begin{tabular}{cccc}
\hline \hline Shape & $\mathrm{x} / \mathbf{L}$ & $\mathbf{Q}[\mathbf{k W}]$ & $M_{p}[\mathrm{~N}-\mathrm{m}]$ \\
\hline $\begin{array}{c}\text { thermal equilibrium, 5 sp. } \\
\text { flap }\end{array}$ & & & \\
oblate spheroid & 0.10 & 0.5 & 0.023 \\
oblate spheroid & 0.10 & 1 & 0.039 \\
oblate spheroid & 0.10 & 2 & 0.061 \\
\hline \hline
\end{tabular}


Table C.2: The pitching moment for the 'large' blunt elliptic cone simulations with freestream conditions listed in Table 3.3 and deposition shape parameters listed in Table 3.4. $\left(L=3 \mathrm{~m}, \mathrm{~T}_{w}=300 \mathrm{~K}\right)$

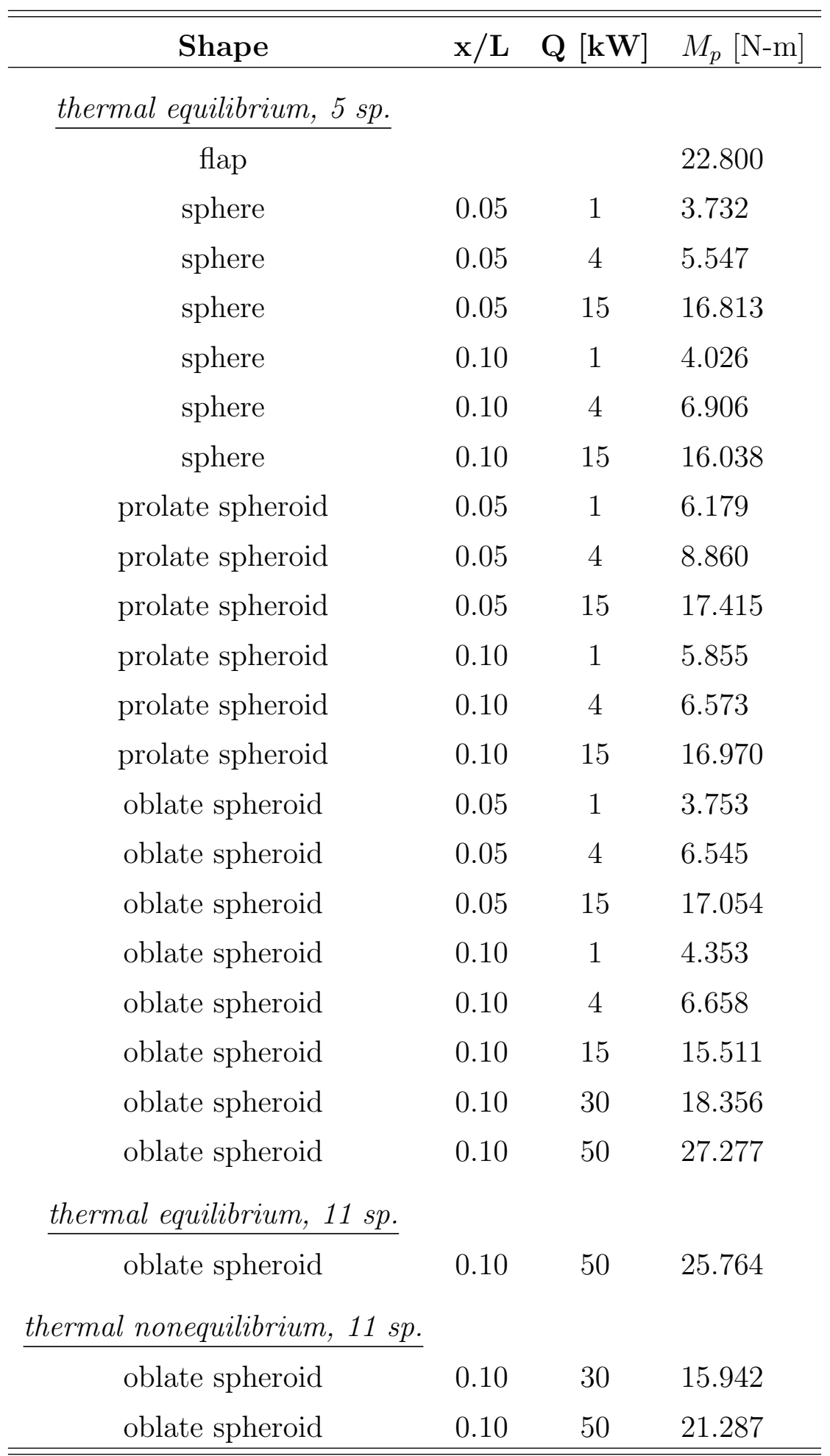


Table C.3: The pitching moment for the 'large' blunt elliptic cone simulations with freestream conditions listed in Table 3.3 and deposition shape parameters listed in Table 3.4. $\left(L=3 \mathrm{~m}, \mathrm{~T}_{w}=1000 \mathrm{~K}\right)$

\begin{tabular}{cccl}
\hline \hline Shape & $\mathbf{x} / \mathbf{L}$ & $\mathbf{Q}[\mathbf{k W}]$ & $M_{p}[\mathrm{~N}-\mathrm{m}]$ \\
\hline $\begin{array}{c}\text { thermal equilibrium, 5 sp. } \\
\text { flap }\end{array}$ & & & \\
oblate spheroid & 0.10 & 0.5 & 0.579 \\
oblate spheroid & 0.10 & 1 & 0.746 \\
oblate spheroid & 0.10 & 4 & 3.216 \\
oblate spheroid & 0.10 & 15 & 10.045 \\
\hline \hline
\end{tabular}

Table C.4: The pitching moment for the 'large' blunt elliptic cone simulations with freestream conditions listed in Table 3.3 and deposition shape parameters listed in Table 3.4. ( $L=3 \mathrm{~m}, \mathrm{~T}_{w}=$ fully radiative)

\begin{tabular}{cccl}
\hline \hline Shape & $\mathbf{x} / \mathbf{L}$ & $\mathbf{Q}[\mathbf{k W}]$ & $M_{p}[\mathrm{~N}-\mathrm{m}]$ \\
\hline $\begin{array}{c}\text { thermal equilibrium, 5 sp. } \\
\text { flap }\end{array}$ & & & \\
oblate spheroid & 0.10 & 4 & 3.023 \\
oblate spheroid & 0.10 & 15 & 10.711 \\
\hline \hline
\end{tabular}

Table C.5: The pitching moment for the 'medium' blunt elliptic cone simulations with freestream conditions listed in Table 3.3 and deposition shape parameters listed in Table 3.5. $\left(L=0.62 \mathrm{~m}, \mathrm{~T}_{w}=300 \mathrm{~K}\right)$

\begin{tabular}{cccc}
\hline \hline Shape & $\mathbf{x} / \mathbf{L}$ & $\mathbf{Q}[\mathrm{kW}]$ & $M_{p}[\mathrm{~N}-\mathrm{m}]$ \\
\hline $\begin{array}{c}\text { thermal equilibrium, 5 sp. } \\
\text { flap }\end{array}$ & & & \\
oblate spheroid & 0.10 & 4 & 0.360 \\
oblate spheroid & 0.10 & 10 & 0.781 \\
oblate spheroid & 0.10 & 15 & 1.083 \\
\hline \hline
\end{tabular}




\section{APPENDIX D}

\section{Air - Mole Fractions}

The following tables list the mole fractions for 11 species thermal equilibrium air. These values are determined using the computational equilibrium composition solver in [115]. Any mole fraction less than $10^{-14}$ is considered within the roundoff error of the simulation and is set to zero $\left(\chi_{s}<10^{-14} \Rightarrow \chi_{s}=0\right)$.

Table D.1: Temperature, total number density, and mole fractions for equilibrium air $\left(p=10^{-3} \mathrm{~atm}\right)$, computed using the computational code in [115].

\begin{tabular}{c|cc|ccc}
\hline \hline & & & \multicolumn{3}{|c}{ Mole Fractions $[\chi]$} \\
$\#$ & $\mathbf{T}[\mathbf{K}]$ & $\mathbf{N}\left[\mathbf{m}^{-3}\right]$ & $\mathrm{N}_{2}$ & $\mathrm{O}_{2}$ & $\mathrm{NO}$ \\
\hline 1 & 2000 & $3.67 \times 10^{21}$ & $7.94 \times 10^{-1}$ & $1.89 \times 10^{-1}$ & $7.54 \times 10^{-3}$ \\
2 & 3000 & $2.45 \times 10^{21}$ & $6.73 \times 10^{-1}$ & $7.62 \times 10^{-3}$ & $8.75 \times 10^{-3}$ \\
3 & 4000 & $1.83 \times 10^{21}$ & $6.32 \times 10^{-1}$ & $4.73 \times 10^{-5}$ & $1.64 \times 10^{-3}$ \\
4 & 5000 & $1.47 \times 10^{21}$ & $2.42 \times 10^{-1}$ & $1.24 \times 10^{-6}$ & $2.78 \times 10^{-4}$ \\
5 & 6000 & $1.22 \times 10^{21}$ & $1.11 \times 10^{-2}$ & $1.03 \times 10^{-7}$ & $2.42 \times 10^{-5}$ \\
6 & 7000 & $1.05 \times 10^{21}$ & $6.39 \times 10^{-4}$ & $2.19 \times 10^{-8}$ & $3.40 \times 10^{-6}$ \\
7 & 8000 & $9.17 \times 10^{20}$ & $5.87 \times 10^{-5}$ & $5.77 \times 10^{-9}$ & $6.32 \times 10^{-7}$ \\
8 & 9000 & $8.15 \times 10^{20}$ & $4.92 \times 10^{-6}$ & $1.25 \times 10^{-9}$ & $9.77 \times 10^{-8}$ \\
9 & 10000 & $7.33 \times 10^{20}$ & $2.00 \times 10^{-7}$ & $1.42 \times 10^{-10}$ & $7.41 \times 10^{-9}$ \\
10 & 11000 & $6.67 \times 10^{20}$ & $4.36 \times 10^{-9}$ & $8.14 \times 10^{-12}$ & $2.85 \times 10^{-10}$ \\
11 & 12000 & $6.11 \times 10^{20}$ & $1.06 \times 10^{-10}$ & $4.03 \times 10^{-13}$ & $1.06 \times 10^{-11}$ \\
\hline \hline
\end{tabular}




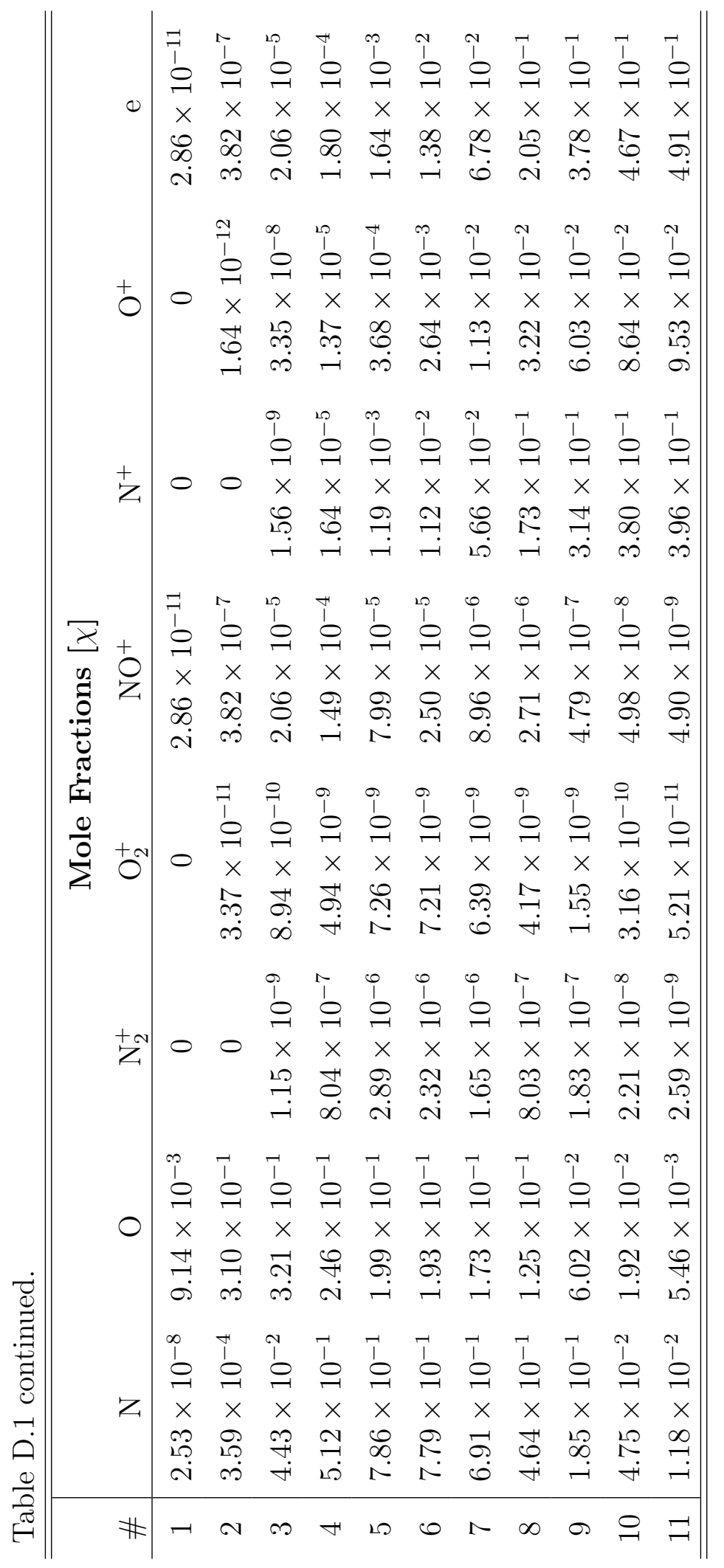


Table D.2: Temperature, total number density, and mole fractions for equilibrium air $(p=1 \mathrm{~atm})$, computed using the computational code in [115].

\begin{tabular}{c|cc|ccc}
\hline \hline & & & \multicolumn{3}{|c}{ Mole Fractions $[\chi]$} \\
$\#$ & $\mathbf{T}[\mathbf{K}]$ & $\mathbf{N}\left[\mathbf{m}^{-3}\right]$ & $\mathrm{N}_{2}$ & $\mathrm{O}_{2}$ & $\mathrm{NO}$ \\
\hline 1 & 2000 & $3.67 \times 10^{24}$ & $7.98 \times 10^{-1}$ & $1.94 \times 10^{-1}$ & $7.87 \times 10^{-3}$ \\
2 & 3000 & $2.45 \times 10^{24}$ & $7.64 \times 10^{-1}$ & $1.51 \times 10^{-1}$ & $4.15 \times 10^{-2}$ \\
3 & 4000 & $1.83 \times 10^{24}$ & $6.80 \times 10^{-1}$ & $2.82 \times 10^{-2}$ & $4.14 \times 10^{-2}$ \\
4 & 5000 & $1.47 \times 10^{24}$ & $6.46 \times 10^{-1}$ & $1.95 \times 10^{-3}$ & $1.80 \times 10^{-2}$ \\
5 & 6000 & $1.22 \times 10^{24}$ & $5.25 \times 10^{-1}$ & $2.26 \times 10^{-4}$ & $7.79 \times 10^{-3}$ \\
6 & 7000 & $1.05 \times 10^{24}$ & $2.56 \times 10^{-1}$ & $3.56 \times 10^{-5}$ & $2.75 \times 10^{-3}$ \\
7 & 8000 & $9.17 \times 10^{23}$ & $6.39 \times 10^{-2}$ & $8.738 \times 10^{-6}$ & $7.95 \times 10^{-4}$ \\
8 & 9000 & $8.15 \times 10^{23}$ & $1.36 \times 10^{-2}$ & $3.11 \times 10^{-6}$ & $2.56 \times 10^{-4}$ \\
9 & 10000 & $7.34 \times 10^{23}$ & $3.37 \times 10^{-3}$ & $1.43 \times 10^{-6}$ & $9.62 \times 10^{-5}$ \\
10 & 11000 & $6.66 \times 10^{23}$ & $9.57 \times 10^{-4}$ & $7.10 \times 10^{-7}$ & $3.95 \times 10^{-5}$ \\
11 & 12000 & $6.10 \times 10^{23}$ & $2.84 \times 10^{-4}$ & $3.56 \times 10^{-7}$ & $1.63 \times 10^{-5}$ \\
\hline \hline
\end{tabular}




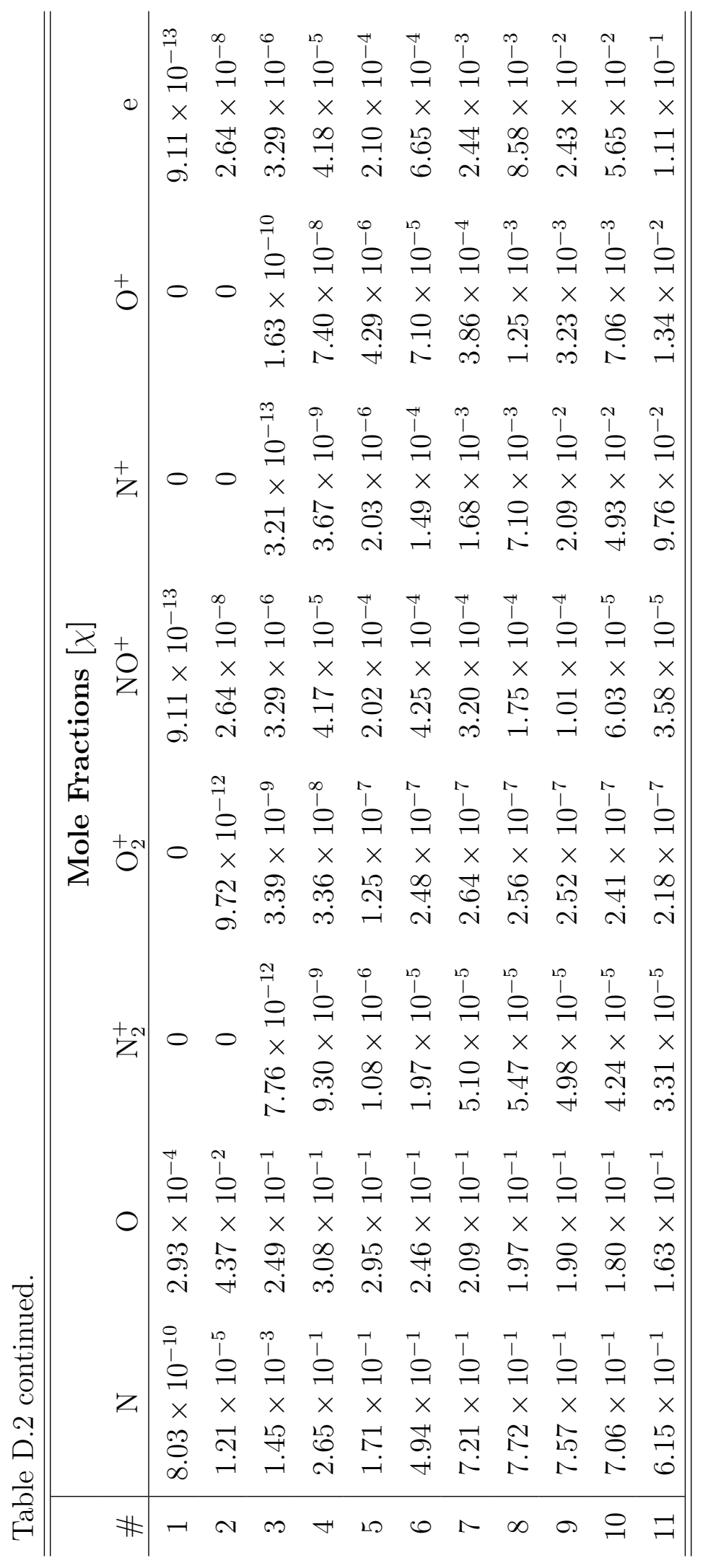




\section{APPENDIX E}

\section{Air - Mole Fraction Angles}

Equation E.1 lists the formulas relating each species mole fraction to their corresponding mole fraction angle for 11 species air, as described in Chapter 5.3.3.

$$
\begin{aligned}
& \beta=\cos ^{-1}\left(\sqrt{\chi_{\mathrm{O}^{+}}}\right) \\
& \psi=\cos ^{-1}\left(\sqrt{\frac{\chi_{\mathrm{N}}+}{\sin ^{2}(\beta)}}\right) \\
& \nu=\cos ^{-1}\left(\sqrt{\frac{\chi_{\mathrm{NO}^{+}}}{\sin ^{2}(\beta) \sin ^{2}(\psi)}}\right) \\
& \zeta=\cos ^{-1}\left(\sqrt{\frac{\chi_{\mathrm{O}_{2}^{+}}}{\sin ^{2}(\beta) \sin ^{2}(\psi) \sin ^{2}(\nu)}}\right) \\
& \mu=\cos ^{-1}\left(\sqrt{\frac{\chi_{\mathrm{N}_{2}^{+}}}{\sin ^{2}(\beta) \sin ^{2}(\psi) \sin ^{2}(\nu) \sin ^{2}(\zeta)}}\right) \\
& \xi=\cos ^{-1}\left(\sqrt{\frac{\chi_{\mathrm{O}}}{\sin ^{2}(\beta) \sin ^{2}(\psi) \sin ^{2}(\nu) \sin ^{2}(\zeta) \sin ^{2}(\mu)}}\right) \\
& \eta=\cos ^{-1}\left(\sqrt{\frac{\chi_{\mathrm{N}}}{\sin ^{2}(\beta) \sin ^{2}(\psi) \sin ^{2}(\nu) \sin ^{2}(\zeta) \sin ^{2}(\mu) \sin ^{2}(\xi)}}\right) \\
& \lambda=\cos ^{-1}\left(\sqrt{\frac{\chi_{\mathrm{NO}}}{\sin ^{2}(\beta) \sin ^{2}(\psi) \sin ^{2}(\nu) \sin ^{2}(\zeta) \sin ^{2}(\mu) \sin ^{2}(\xi) \sin ^{2}(\eta)}}\right) \\
& \theta=\tan ^{-1}\left(\sqrt{\frac{\chi_{\mathrm{N}_{2}}}{\chi_{\mathrm{O}_{2}}}}\right)
\end{aligned}
$$




\section{APPENDIX F}

\section{Polynomial Response Model}

The PRS models developed in Chapter V have a total number of coefficients $=\frac{(k+n) !}{(k ! n !)}$, where $k$ is the number dimensions and $n$ is the order or degree of the polynomial. The variables and constant coefficients developed using this procedure are explained and listed below for both three species argon and eleven species air.

\section{F.1 Variables}

For three species argon, there are three variables, $\mathrm{E} / N, \chi_{\mathrm{Ar}}$, and $\chi_{\mathrm{Ar}^{+}}$. Since the mole fractions already range from 0 to 1 , they are used directly in the model. However, the flow solver provides the reduced electric field in units of $\mathrm{V}-\mathrm{m}^{2}$. The typical strength of an electric field is between a few hundredths to a few hundred Townsend $\left(1 \mathrm{Td}=10^{-17} \mathrm{~V}-\mathrm{cm}^{2}\right)$. This means the reduced electric field parameter varies between $10^{-23} \mathrm{~V}-\mathrm{m}^{2}$ and $10^{-19} \mathrm{~V}-\mathrm{m}^{2}$. In order to improve the accuracy of the model, the reduced electric field variable is scaled from 0 to 1 , where 0 is functionally equivalent to $0.01 \mathrm{Td}$ and 1 is $100 \mathrm{Td}$. This is accomplished by first converting the reduced electric field from the flow solver into units of $\mathrm{V}-\mathrm{cm}^{2}$, and then applying a logarithm of base 10 . The resulting variable is scaled so: $-19 \rightarrow 0$ and $-15 \rightarrow 1$. Table F.1 lists some additional examples. This does not mean that the reduced 
electric field must fall between $0.01 \mathrm{Td}$ and $100 \mathrm{Td}$, rather that this is simply the range of the DOE used when developing the model. The same methodology also applies for the eleven species air models.

Table F.1: Examples of scaling the normalized electric field.

\begin{tabular}{c|cc|c}
\hline \hline Flow Solver $\left[\mathrm{V}-\mathrm{m}^{2}\right]$ & \multicolumn{2}{|c|}{ Intermediate Steps } & Model Variable \\
\hline $10^{-23}$ & $10^{-19} \mathrm{~V}-\mathrm{cm}^{2}$ & -19 & 0 \\
$5 \times 10^{-23}$ & $5 \times 10^{-19} \mathrm{~V}-\mathrm{cm}^{2}$ & -18.3 & 0.175 \\
$10^{-22}$ & $10^{-18} \mathrm{~V}-\mathrm{cm}^{2}$ & -18 & 0.25 \\
$5 \times 10^{-22}$ & $5 \times 10^{-18} \mathrm{~V}-\mathrm{cm}^{2}$ & -17.3 & 0.425 \\
$10^{-21}$ & $10^{-17} \mathrm{~V}-\mathrm{cm}^{2}$ & -17 & 0.5 \\
$5 \times 10^{-21}$ & $5 \times 10^{-17} \mathrm{~V}-\mathrm{cm}^{2}$ & -16.3 & 0.675 \\
$10^{-20}$ & $10^{-16} \mathrm{~V}-\mathrm{cm}^{2}$ & -16 & 0.75 \\
$5 \times 10^{-20}$ & $5 \times 10^{-16} \mathrm{~V}-\mathrm{cm}^{2}$ & -15.3 & 0.925 \\
$10^{-19}$ & $10^{-15} \mathrm{~V}-\mathrm{cm}^{2}$ & -15 & 1 \\
\hline \hline
\end{tabular}

\section{F.2 Constant Coefficients}

The following tables list the constant coefficients for the $3^{\text {rd }}$ Order PRS model of argon and the $2^{\text {nd }}$ and $3^{\text {rd }}$ Order models of air. 
Table F.2: Constant coefficients for three species argon ( $3^{\text {rd }}$ Order PRS).

$$
[\alpha<=0.1 \%]
$$

\begin{tabular}{|c|c|c|c|}
\hline \multicolumn{2}{|c|}{ Coefficient } & \multicolumn{2}{|r|}{ Variable } \\
\hline$c_{0}=$ & -1057.327 & $d_{0}=$ & \\
\hline$c_{1}=$ & -139.94 & $d_{1}=$ & $\mathrm{E} / N$ \\
\hline$c_{2}=$ & 1249.183 & $d_{2}=$ & $\chi_{\mathrm{Ar}}$ \\
\hline$c_{3}=$ & -2579.669 & $d_{3}=$ & $\chi_{\mathrm{Ar}^{+}}$ \\
\hline$c_{4}=$ & 40.664 & $d_{4}=$ & $(\mathrm{E} / N)(\mathrm{E} / N)$ \\
\hline$c_{5}=$ & 429.334 & $d_{5}=$ & $(\mathrm{E} / N)\left(\chi_{\mathrm{Ar}}\right)$ \\
\hline$c_{6}=$ & -702.205 & $d_{6}=$ & $(\mathrm{E} / N)\left(\chi_{\mathrm{Ar}^{+}}\right)$ \\
\hline$c_{7}=$ & 1135.904 & $d_{7}=$ & $\left(\chi_{\mathrm{Ar}}\right)\left(\chi_{\mathrm{Ar}}\right)$ \\
\hline$c_{8}=$ & -161.566 & $d_{8}=$ & $\left(\chi_{\mathrm{Ar}}\right)\left(\chi_{\mathrm{Ar}^{+}}\right)$ \\
\hline$c_{9}=$ & -2418.255 & $d_{9}=$ & $\left(\chi_{\mathrm{Ar}^{+}}\right)\left(\chi_{\mathrm{Ar}^{+}}\right)$ \\
\hline$c_{10}=$ & -5.944 & $d_{10}=$ & $(\mathrm{E} / N)(\mathrm{E} / N)(\mathrm{E} / N)$ \\
\hline$c_{11}=$ & -36.379 & $d_{11}=$ & $(\mathrm{E} / N)(\mathrm{E} / N)\left(\chi_{\mathrm{Ar}}\right)$ \\
\hline$c_{12}=$ & -32.209 & $d_{12}=$ & $(\mathrm{E} / N)(\mathrm{E} / N)\left(\chi_{\mathrm{Ar}^{+}}\right)$ \\
\hline$c_{13}=$ & -283.146 & $d_{13}=$ & $(\mathrm{E} / N)\left(\chi_{\mathrm{Ar}}\right)\left(\chi_{\mathrm{Ar}}\right)$ \\
\hline$c_{14}=$ & 560.087 & $d_{14}=$ & $(\mathrm{E} / N)\left(\chi_{\mathrm{Ar}}\right)\left(\chi_{\mathrm{Ar}^{+}}\right)$ \\
\hline$c_{15}=$ & -1262.358 & $d_{15}=$ & $(\mathrm{E} / N)\left(\chi_{\mathrm{Ar}^{+}}\right)\left(\chi_{\mathrm{Ar}^{+}}\right)$ \\
\hline$c_{16}=$ & -1344.271 & $d_{16}=$ & $\left(\chi_{\mathrm{Ar}}\right)\left(\chi_{\mathrm{Ar}}\right)\left(\chi_{\mathrm{Ar}}\right)$ \\
\hline$c_{17}=$ & 2253.127 & $d_{17}=$ & $\left(\chi_{\mathrm{Ar}}\right)\left(\chi_{\mathrm{Ar}}\right)\left(\chi_{\mathrm{Ar}^{+}}\right)$ \\
\hline$c_{18}=$ & -2414.827 & $d_{18}=$ & $\left(\chi_{\mathrm{Ar}}\right)\left(\chi_{\mathrm{Ar}^{+}}\right)\left(\chi_{\mathrm{Ar}^{+}}\right)$ \\
\hline$c_{19}=$ & -3.433 & $d_{19}=$ & $\left(\chi_{\mathrm{Ar}^{+}}\right)\left(\chi_{\mathrm{Ar}^{+}}\right)\left(\chi_{\mathrm{Ar}^{+}}\right)$ \\
\hline
\end{tabular}


Table F.3: Constant coefficients for eleven species air ( $2^{\text {nd }}$ Order PRS). $[\alpha<=0.1 \%]$

\begin{tabular}{lr|rr|rr|lr}
\hline \hline$c_{0}$ & 113.069 & $c_{20}$ & -405.335 & $c_{40}$ & -133.910 & $c_{60}$ & 74.992 \\
$c_{1}$ & -168.062 & $c_{21}$ & 197.847 & $c_{41}$ & -179.857 & $c_{61}$ & -26.832 \\
$c_{2}$ & -135.172 & $c_{22}$ & 132.532 & $c_{42}$ & 45.653 & $c_{62}$ & 8.159 \\
$c_{3}$ & -64.721 & $c_{23}$ & 8.562 & $c_{43}$ & -45.334 & $c_{63}$ & 0.261 \\
$c_{4}$ & -172.628 & $c_{24}$ & -54.425 & $c_{44}$ & 17.540 & $c_{64}$ & 0.050 \\
$c_{5}$ & -38.302 & $c_{25}$ & 53.062 & $c_{45}$ & -80.197 & $c_{65}$ & 0.051 \\
$c_{6}$ & -100.893 & $c_{26}$ & -80.634 & $c_{46}$ & -48.880 & $c_{66}$ & 0.052 \\
$c_{7}$ & 488.760 & $c_{27}$ & -18.199 & $c_{47}$ & -75.379 & $c_{67}$ & 0.031 \\
$c_{8}$ & 191.227 & $c_{28}$ & 19.699 & $c_{48}$ & -23.504 & $c_{68}$ & 0.125 \\
$c_{9}$ & 232.748 & $c_{29}$ & -84.724 & $c_{49}$ & 2.172 & $c_{69}$ & 0.019 \\
$c_{10}$ & -156.137 & $c_{30}$ & 45.794 & $c_{50}$ & -89.661 & $c_{70}$ & -0.011 \\
$c_{11}$ & -131.833 & $c_{31}$ & -43.196 & $c_{51}$ & -116.369 & $c_{71}$ & 0.014 \\
$c_{12}$ & 2.895 & $c_{32}$ & 18.886 & $c_{52}$ & 164.844 & $c_{72}$ & 0.174 \\
$c_{13}$ & 166.649 & $c_{33}$ & -62.788 & $c_{53}$ & 106.208 & $c_{73}$ & -0.007 \\
$c_{14}$ & 167.274 & $c_{34}$ & -17.761 & $c_{54}$ & 85.439 & $c_{74}$ & 0.011 \\
$c_{15}$ & 167.102 & $c_{35}$ & -153.057 & $c_{55}$ & 71.394 & $c_{75}$ & -0.076 \\
$c_{16}$ & 167.368 & $c_{36}$ & -90.268 & $c_{56}$ & 18.862 & $c_{76}$ & -0.046 \\
$c_{17}$ & 167.430 & $c_{37}$ & 340.879 & $c_{57}$ & -26.629 & $c_{77}$ & -0.066 \\
$c_{18}$ & -648.352 & $c_{38}$ & 184.811 & $c_{58}$ & 43.091 & & \\
$c_{19}$ & -280.588 & $c_{39}$ & 101.655 & $c_{59}$ & 33.615 & & \\
\hline \hline & & & & & & & \\
\hline
\end{tabular}


Table F.4: Constant coefficients for eleven species air ( $3^{\text {rd }}$ Order PRS). $[\alpha<=0.1 \%]$

\begin{tabular}{|c|c|c|c|c|c|c|c|c|c|}
\hline$c_{0}$ & 133.324 & $c_{30}$ & 90.954 & $c_{60}$ & -88.893 & $c_{90}$ & 369.494 & $c_{120}$ & -13.100 \\
\hline$c_{1}$ & -290.579 & $c_{31}$ & 94.215 & $c_{61}$ & -1.699 & $c_{91}$ & -44.710 & $c_{121}$ & 215.346 \\
\hline$c_{2}$ & -47.628 & $c_{32}$ & 6.911 & $c_{62}$ & -42.745 & $c_{92}$ & 66.667 & $c_{122}$ & 50.120 \\
\hline$c_{3}$ & 13.011 & $c_{33}$ & -14.617 & $c_{63}$ & -0.927 & $c_{93}$ & 31.361 & $c_{123}$ & -13.140 \\
\hline$c_{4}$ & -227.561 & $c_{34}$ & 198.368 & $c_{64}$ & -0.613 & $c_{94}$ & -77.489 & $c_{124}$ & -135.580 \\
\hline$c_{5}$ & -175.978 & $c_{35}$ & -336.836 & $c_{65}$ & 0.398 & $c_{95}$ & -17.767 & $c_{125}$ & 71.029 \\
\hline$c_{6}$ & -141.487 & $c_{36}$ & -351.557 & $c_{66}$ & 0.574 & $c_{96}$ & -191.789 & $c_{126}$ & -39.417 \\
\hline$c_{7}$ & 558.765 & $c_{37}$ & 368.341 & $c_{67}$ & 0.599 & $c_{97}$ & -99.938 & $c_{127}$ & 115.282 \\
\hline$c_{8}$ & 147.117 & $c_{38}$ & 146.907 & $c_{68}$ & 1.219 & $c_{98}$ & 164.242 & $c_{128}$ & 54.889 \\
\hline$c_{9}$ & 304.998 & $c_{39}$ & 297.559 & $c_{69}$ & 0.278 & $c_{99}$ & 324.399 & $c_{129}$ & -1.056 \\
\hline$c_{10}$ & -169.924 & $c_{40}$ & -158.86 & $c_{70}$ & 0.012 & $c_{100}$ & 235.022 & $c_{130}$ & -0.539 \\
\hline$c_{11}$ & -129.201 & $c_{41}$ & 13.508 & $c_{71}$ & -0.578 & $c_{101}$ & 347.386 & $c_{131}$ & -0.017 \\
\hline$c_{12}$ & 95.863 & $c_{42}$ & 136.578 & $c_{72}$ & -1.031 & $c_{102}$ & 312.398 & $c_{132}$ & 0.095 \\
\hline$c_{13}$ & 244.729 & $c_{43}$ & -258.320 & $c_{73}$ & 0.050 & $c_{103}$ & -697.177 & $c_{133}$ & 0.155 \\
\hline$c_{14}$ & -35.972 & $c_{44}$ & 129.338 & $c_{74}$ & 0.044 & $c_{104}$ & -434.793 & $c_{134}$ & 0.233 \\
\hline$c_{15}$ & 378.033 & $c_{45}$ & -245.368 & $c_{75}$ & -0.695 & $c_{105}$ & -463.284 & $c_{135}$ & 0.077 \\
\hline$c_{16}$ & 267.944 & $c_{46}$ & -187.212 & $c_{76}$ & -0.343 & $c_{106}$ & 96.555 & $c_{136}$ & 0.140 \\
\hline$c_{17}$ & 303.934 & $c_{47}$ & 83.029 & $c_{77}$ & 0.550 & $c_{107}$ & -126.172 & $c_{137}$ & -0.138 \\
\hline$c_{18}$ & -1008.692 & $c_{48}$ & 75.279 & $c_{78}$ & 0.507 & $c_{108}$ & -89.334 & $c_{138}$ & -0.866 \\
\hline$c_{19}$ & -327.567 & $c_{49}$ & -30.944 & $c_{79}$ & -93.431 & $c_{109}$ & -66.322 & $c_{139}$ & 0.064 \\
\hline$c_{20}$ & -671.314 & $c_{50}$ & 178.022 & $c_{80}$ & -91.823 & $c_{110}$ & -102.635 & $c_{140}$ & 0.038 \\
\hline$c_{21}$ & 275.655 & $c_{51}$ & 190.119 & $c_{81}$ & -92.588 & $c_{111}$ & 187.180 & $c_{141}$ & -0.093 \\
\hline$c_{22}$ & 282.290 & $c_{52}$ & 102.843 & $c_{82}$ & -94.274 & $c_{112}$ & 134.595 & $c_{142}$ & -0.142 \\
\hline$c_{23}$ & -155.204 & $c_{53}$ & 142.895 & $c_{83}$ & -94.312 & $c_{113}$ & 36.981 & $c_{143}$ & 0.425 \\
\hline$c_{24}$ & -150.085 & $c_{54}$ & -77.391 & $c_{84}$ & 509.26 & $c_{114}$ & -51.653 & $c_{144}$ & 55.878 \\
\hline$c_{25}$ & -128.509 & $c_{55}$ & -178.456 & $c_{85}$ & 37.074 & $c_{115}$ & 138.872 & $c_{145}$ & -53.777 \\
\hline$c_{26}$ & 137.274 & $c_{56}$ & -76.205 & $c_{86}$ & 362.347 & $c_{116}$ & 22.808 & $c_{146}$ & 163.214 \\
\hline$c_{27}$ & -151.073 & $c_{57}$ & 5.125 & $c_{87}$ & -183.192 & $c_{117}$ & 9.705 & $c_{147}$ & -152.738 \\
\hline$c_{28}$ & 190.860 & $c_{58}$ & 142.057 & $c_{88}$ & -163.425 & $c_{118}$ & -284.265 & $c_{148}$ & 101.899 \\
\hline$c_{29}$ & 16.627 & $c_{59}$ & 27.584 & $c_{89}$ & 44.565 & $c_{119}$ & -80.404 & $c_{149}$ & -417.194 \\
\hline
\end{tabular}




\begin{tabular}{|c|c|c|c|c|c|c|c|c|c|}
\hline$c_{150}$ & -43.465 & 180 & 175.725 & $c_{210}$ & -150.659 & $c_{240}$ & 0.062 & $c_{270}$ & -0.070 \\
\hline$c_{151}$ & 193.367 & $c_{181}$ & -498.404 & $c_{211}$ & 2.533 & $c_{241}$ & -0.093 & $c_{271}$ & -0.020 \\
\hline$c_{152}$ & 82.364 & $c_{182}$ & 84.022 & $c_{212}$ & 435.691 & $c_{242}$ & -0.078 & $c_{272}$ & 0.252 \\
\hline$c_{153}$ & -84.976 & $c_{183}$ & -425.708 & $c_{213}$ & 333.719 & $c_{243}$ & 0.132 & $c_{273}$ & -0.104 \\
\hline$c_{154}$ & -257.753 & $c_{184}$ & -0.675 & $c_{214}$ & -184.642 & $c_{244}$ & -56.685 & $c_{274}$ & 0.052 \\
\hline$c_{155}$ & -281.982 & $c_{185}$ & 0.019 & $c_{215}$ & 514.040 & $c_{245}$ & 312.096 & $c_{275}$ & -0.029 \\
\hline$c_{156}$ & -113.287 & $c_{186}$ & 0.133 & $c_{216}$ & 52.475 & $c_{246}$ & -109.993 & $c_{276}$ & -0.168 \\
\hline$c_{157}$ & 119.780 & $c_{187}$ & 0.312 & $c_{217}$ & 216.490 & $c_{247}$ & 191.511 & $c_{277}$ & -0.165 \\
\hline$c_{158}$ & 633.591 & $c_{188}$ & 0.212 & $c_{218}$ & 185.787 & $c_{248}$ & 260.413 & $c_{278}$ & -0.102 \\
\hline$c_{159}$ & -260.375 & $c_{189}$ & 0.321 & $c_{219}$ & -378.221 & $c_{249}$ & -380.758 & $c_{279}$ & 0.224 \\
\hline$c_{160}$ & 120.097 & $c_{190}$ & -0.011 & $c_{220}$ & -201.505 & $c_{250}$ & 115.103 & $c_{280}$ & -150.364 \\
\hline$c_{161}$ & -27.060 & $c_{191}$ & -0.050 & $c_{221}$ & -226.978 & $c_{251}$ & & $c_{281}$ & -319.163 \\
\hline$c_{162}$ & & $c_{19}$ & & $c_{222}$ & & & 21 & $c_{2}$ & 597 \\
\hline$c_{163}$ & 53.514 & $c_{193}$ & -0.209 & $c_{223}$ & 171.618 & $c^{253}$ & 137.822 & $c_{283}$ & 91.540 \\
\hline$c_{164}$ & 265.129 & $c_{194}$ & -0.005 & $c_{224}$ & -562.41 & $c_{254}$ & -249.106 & $c_{284}$ & 161.182 \\
\hline$c_{165}$ & 96.850 & $c_{195}$ & 0.016 & $c_{225}$ & -417.695 & $c_{255}$ & -409.748 & $c_{285}$ & 249.369 \\
\hline$c_{166}$ & -272.878 & $c_{196}$ & -0.111 & $c_{226}$ & -13.343 & $c_{256}$ & -231.720 & $c_{286}$ & 69.291 \\
\hline$c_{167}$ & -536.231 & $c_{197}$ & -0.048 & $c_{227}$ & 30.156 & $c_{257}$ & 43.527 & $c_{287}$ & -180.451 \\
\hline$c_{168}$ & 147.951 & $c_{198}$ & 08 & $c_{228}$ & 216 & $c_{258}$ & -632 & $c_{288}$ & .497 \\
\hline 109 & 136.437 & $c_{1}$ & -146.127 & $c_{2}$ & 0.116 & $\sigma_{2}$ & -69.844 & $c_{289}$ & 158.175 \\
\hline 100 & & $c_{200}$ & -426.684 & $c_{230}$ & -0.181 & $c_{260}$ & & $c_{290}$ & 66.826 \\
\hline$c_{171}$ & -358.524 & $c_{201}$ & 58.482 & $c_{231}$ & 0.019 & $c_{261}$ & 62.913 & $c_{291}$ & -62.336 \\
\hline$c_{172}$ & -332.070 & $c_{202}$ & 38.222 & $c_{232}$ & 0.165 & $c_{262}$ & 213.881 & $c_{292}$ & 261.307 \\
\hline$c_{173}$ & -245.980 & $c_{203}$ & 108.612 & $c_{233}$ & 0.110 & $c_{263}$ & -35.094 & $c_{293}$ & -0.008 \\
\hline$c_{174}$ & 680.852 & $c_{204}$ & 767.575 & $c_{234}$ & 0.193 & $c_{264}$ & -99.362 & $c_{294}$ & -0.085 \\
\hline$c_{175}$ & 128.018 & $c_{205}$ & 58.390 & $c_{235}$ & 0.070 & $c_{265}$ & -0.061 & $c_{295}$ & 0.063 \\
\hline$c_{176}$ & -181.645 & $c_{206}$ & 249.677 & $c_{236}$ & -0.027 & $c_{266}$ & -0.307 & $c_{296}$ & 0.005 \\
\hline$c_{177}$ & 447.508 & $c_{207}$ & -465.176 & $c_{237}$ & -0.124 & $c_{267}$ & 0.122 & $c_{297}$ & 0.073 \\
\hline$c_{178}$ & 33.448 & $c_{208}$ & -335.907 & $c_{238}$ & 0.029 & $c_{268}$ & -0.007 & $c_{298}$ & 0.297 \\
\hline$c_{179}$ & 493.320 & $c_{209}$ & 292.245 & $c_{239}$ & 0.020 & $c_{269}$ & 0.046 & $c_{299}$ & 0.234 \\
\hline
\end{tabular}




\begin{tabular}{rr|rr|lr|lr|rr}
\multicolumn{2}{l}{ (continued) } \\
\hline \hline$c_{300}$ & -0.134 & $c_{314}$ & -0.298 & $c_{328}$ & 0.189 & $c_{342}$ & 0 & $c_{356}$ & 0 \\
$c_{301}$ & -0.016 & $c_{315}$ & -0.058 & $c_{329}$ & -0.002 & $c_{343}$ & 0 & $c_{357}$ & 0 \\
$c_{302}$ & -0.630 & $c_{316}$ & 0.060 & $c_{330}$ & 0 & $c_{344}$ & 0.001 & $c_{358}$ & 0 \\
$c_{303}$ & 0.016 & $c_{317}$ & 0.099 & $c_{331}$ & 0 & $c_{345}$ & 0 & $c_{359}$ & 0 \\
$c_{304}$ & 0.124 & $c_{318}$ & 0.157 & $c_{332}$ & 0 & $c_{346}$ & 0 & $c_{360}$ & -0.001 \\
$c_{305}$ & -0.277 & $c_{319}$ & 0.476 & $c_{333}$ & 0 & $c_{347}$ & 0 & $c_{361}$ & 0 \\
$c_{306}$ & -0.004 & $c_{320}$ & 0.005 & $c_{334}$ & 0 & $c_{348}$ & 0 & $c_{362}$ & 0 \\
$c_{307}$ & -0.003 & $c_{321}$ & -0.029 & $c_{335}$ & 0 & $c_{349}$ & 0 & $c_{363}$ & 0 \\
$c_{308}$ & -11.475 & $c_{322}$ & -0.176 & $c_{336}$ & 0 & $c_{350}$ & 0 & & \\
$c_{309}$ & -115.090 & $c_{323}$ & -0.272 & $c_{337}$ & 0 & $c_{351}$ & 0 & & \\
$c_{310}$ & 48.248 & $c_{324}$ & 0.047 & $c_{338}$ & 0 & $c_{352}$ & 0 & & \\
$c_{311}$ & 142.297 & $c_{325}$ & 0.010 & $c_{339}$ & 0 & $c_{353}$ & 0 & & \\
$c_{312}$ & -18.405 & $c_{326}$ & -0.048 & $c_{340}$ & 0 & $c_{354}$ & -0.001 & & \\
$c_{313}$ & 4.677 & $c_{327}$ & -0.11 & $c_{341}$ & 0 & $c_{355}$ & 0 & & \\
\hline \hline
\end{tabular}




\section{BIBLIOGRAPHY}




\section{BIBLIOGRAPHY}

[1] Allen, E. H., "The Case for Near Space," Aerospace America, February 2006, pp. 31.

[2] McClinton, C. R., "X-43 Scramjet Power Breaks the Hypersonic Barrier Dryden Lectureship in Research for 2006," AIAA 2006-0001, January 2006, presented at the 44th AIAA Aerospace Sciences Meeting and Exhibit, Reno, NV.

[3] Borrell, B., "Electromagnetic Railgun Blasts Off," Technology Review, February 2008, [Online article], [accessed 15 May 2009].

[4] Prandtl, L. and Tietjens, O. G., Fundamentals of Hydro- and Aeromechanics, McGraw-Hill, 1934, translated by L. Rosenhead.

[5] Maxwell, J. C., "On Physical Lines of Force," Philosophical Magazine Series 4 (1851-1875), Vol. 21, No. 139, March 1861, pp. 161-175.

[6] Alfvén, H., "Existence of Electromagnetic-Hydrodynamic Waves," Nature, Vol. 150, October 1942, pp. 405-406.

[7] Kantrowitz, A. R., "A Survey of Physical Phenomena Occurring in Flight at Extreme Speeds," Proceedings of the Conference on High-Speed Aeronautics, edited by A. Ferri, N. J. Hoff, and P. A. Libby (Polytechnic Institute of Brooklyn, New York, 1955), pp. 335-339.

[8] Resler, E. L. and Sears, W. R., "The Prospects for Magneto-Aerodynamics," Journal of Aeronatuical Sciences, Vol. 25, No. 4, April 1958, pp. 235-245, 258.

[9] Resler, E. L. and Sears, W. R., "The Prospects for Magneto-Aerodynamics: Correction and Addition," Journal of Aero/Space Sciences, Vol. 26, No. 5, May 1959, pp. 319.

[10] Bush, W. B., "Magnetohydrodynamic - Hypersonic Flow Past a Blunt Body," Journal of Aero/Space Sciences, Vol. 25, No. 11, November 1958, pp. 685-690.

[11] Bush, W. B., "The Stagnation-Point Boundary Layer in the Presence of an Applied Magnetic Field," Journal of Aero/Space Sciences, Vol. 28, No. 8, August 1961, pp. 610-611. 
[12] Poggie, J. and Gaitonde, D. V., "Magnetic control of flow past a blunt body: Numerical Validation and Exploration," Physics of Fluids, Vol. 14, No. 5, May 2002, pp. 1720-1731.

[13] Coakley, J. F. and Porter, R. W., "Time-Dependent Numerical Analysis of MHD Blunt Body Problem," AIAA Journal, Vol. 9, No. 8, August 1971, pp. $1624-1626$.

[14] Ziemer, R. W., "Experimental Investigations in Magneto-Aerodynamics," Journal of American Rocket Society, Vol. 29, No. 9, September 1959, pp. 642.

[15] Wilkinson, B., "Magnetohydrodynamic Effects on Stagnation-Point Heat Transfer from Partially Ionized Nonequilibrium Gases in Supersonic Flow," Proceedings from the 3rd Engineering Aspects of Magnetohydrodynamics Symposium, edited by N. W. Mather and G. W. Sutton (Gordon and Breach, New York, 1964), pp. 413-438.

[16] Kranc, S., Yuen, M. C., and Cambel, A. B., "Experimental Investigation of Magnetoaerodynamic Flow around Blunt Bodies," Tech. Rep. NASA CR-1392, National Aeronautics and Space Administration, Washington, D.C., August 1969 .

[17] Nowak, R. J., Kran, S., Porter, R. W., Yuen, M. C., and Cambel, A. B., "Magnetogasdynamic Re-Entry Phenomena," Journal of Spacecraft and Rockets, Vol. 4, No. 11, November 1967, pp. 1538-1542.

[18] Nowak, R. J. and Yuen, M. C., "Heat Transfer to a Hemispherical Body in Supersonic Argon Plasma," AIAA Journal, Vol. 11, No. 11, November 1973, pp. 1463-1464.

[19] Porter, R. W. and Cambel, A. B., "Hall Effect in Flight Magnetogasdynamics," AIAA Journal, Vol. 5, No. 12, December 1967, pp. 2208-2213.

[20] Romig, M. F., "The Influence of Electric and Magnetic Fields on Heat Transfer to Electrically Conducting Fluids," Advances in Heat Transfer, Vol. 1, edited by T. F. Irvine and J. P. Hartnett (Academic Press, New York, 1964), pp. $267-354$.

[21] Padilla, J. F., Assessement of Gas-Surface Interaction Models for Computation of Rarefied Hypersonic Flows, 2008, PhD thesis, Department of Aerospace Engineering, University of Michigan.

[22] Fomin, V. M., Tretyakov, P. K., and Taran, J.-P., "Flow Control using Various Plasma and Aerodynamic Approaches," Aerospace Science and Technology, Vol. 8, No. 5, July 2004, pp. 411-421.

[23] Shang, J. S., Surzhikov, S. T., Kimmel, R., Gaitonde, D., Menart, J., and Hayes, J., "Mechanisms of Plasma Actuators for Hypersonic Flow Control," Progress in Aerospace Sciences, Vol. 41, No. 8, November 2005, pp. 642-668. 
[24] Bityurin, V., Bocharov, A., and Lineberry, J., "MHD Flow Control in Hypersonic Flight," AIAA 2005-3225, May 2005, presented at the 13th Inernational Space Planes and Hypersonic Systems Technologies Conference, Capua, Italy.

[25] Palmer, G., "Magnetic Field Effects on the Computed Flow over a Mars Return Aerobrake," Journal of Thermophysics and Heat Transfer, Vol. 7, No. 2, AprilJune 1993, pp. 294-301.

[26] Lineberry, J. T., Bityurin, V. A., Bocharov, A. N., Baranov, D. S., Vatazhin, A. B., Kopchenov, V. I., Gouskov, O., Alferov, V., and Boushmin, A. S., "Cylinder with Current in Hypersonic Flow," Proceedings from the 3rd Workshop on Magneto-Plasma Aerodynamics in Aerospace Applications, Moscow, Russia, April 2001, pp. 15-25.

[27] Takizawa, Y., Sato, S., Abe, T., and Konigorski, D., "Electro-Magnetic Effect on Shock Layer Structure in Reentry-Related High-Enthalpy Flow," AIAA 2004-2162, June 2004, presented at the 35th AIAA Plasmadynamics and Lasers Conference, Portland, OR.

[28] Kimmel, R., Hayes, J., Menart, J., and Shang, J., "Supersonic Plasma Flow Control Experiments," Tech. Rep. ARFL-VA-WP-TR-2006-3006, U.S. Air Force Research Laboratory, Wright-Patterson AFB, OH, December 2005, cleared for public release, AFRL/WS 06-0097.

[29] Matsuda, A., Kawamura, M., Takizawa, Y., Otsu, H., Konigorski, D., Sato, S., and Abe, T., "Experimental Investigation of the Hall Effect for the Interaction between the Weakly-Ionized Plasma Flow and Magnetic Body," AIAA 200\%1437, January 2007, presented at the 45th Aerospace Sciences Meeting and Exhibit, Reno, NV.

[30] Gülhan, A., Esser, B., Koch, U., Siebe, F., Riehmer, J., Giordano, D., and Konigorski, D., "Experimental Verification of Heat-Flux Mitigation by Electromagnetic Fields in Partially-Ionized-Argon Flows," Journal of Spacecraft and Rockets, Vol. 46, No. 2, March-April 2009, pp. 274-283.

[31] Mason, W. H. and Lee, J., "Aerodynamically Blunt and Sharp Bodies," Journal of Spacecraft and Rockets, Vol. 31, No. 3, May 1994, pp. 378-382.

[32] Santos, W. F. N. and Lewis, M. J., "Aerothermodynamic Performance Analysis of Hypersonic Flow on Power Law Leading Edges," Journal of Spacecraft and Rockets, Vol. 42, No. 4, July-August 2005, pp. 588-597.

[33] Shang, J. S., Hayes, J., and Menart, J., "Hypersonic Flow over a Blunt Body with Plasma Injection," Journal of Spacecraft and Rockets, Vol. 39, No. 3, May-June 2002, pp. 367-375.

[34] Kremeyer, K., Sebastian, K., and Shu, C.-W., "Computational Study of Shock Mitigation and Drag Reduction by Pulsed Energy Lines," AIAA Journal, Vol. 44, No. 8, August 2006, pp. 1720-1731. 
[35] Yan, H. and Gaitonde, D., "Control of Edney IV Interaction by Energy Pulse," AIAA 2006-562, January 2006, presented at the 44th AIAA Aerospace Sciences Meeting and Exhibit, Reno, NV.

[36] Macheret, S. O., Shneider, M. N., and Miles, R. B., "Magnetohydrodynamic Control of Hypersonic Flows and Scramjet Inlets Using Electron Beam Ionization," AIAA Journal, Vol. 40, No. 1, January 2002, pp. 74-81.

[37] Shang, J. S., Huang, H. Y., Surzhikov, S. T., and Gaitonde, D. V., "Hypersonic Flow Control Utilizing Electronmaggetic-Aerodynamic Interaction," AIAA 2008-2606, April 2008, presented at the 15th AIAA International Space Planes and Hypersonic Systems and Technologies Conference, Dayton, OH.

[38] Girgis, I. G., Shneider, M. N., Macheret, S. O., Brown, G. L., and Miles, R. B., "Creation of Steering Moments in Supersonic Flow by Off-Axis Plasma Heat Addition," AIAA 2002-129, January 2002, presented at the 40th AIAA Aerospace Sciences Meeting and Exhibit, Reno, NV.

[39] Gnemmi, P., Charon, R., Dupéroux, J.-P., and George, A., "Feasibility Study for Steering a Supersonic Projectile by a Plasma Actuator," AIAA Journal, Vol. 46, No. 6, June 2008, pp. 1308-1317.

[40] Menart, J., Stanfield, S., Shang, J., Kimmel, R., and Hayes, J., "Study of Plasma Electrode Arrangements for Optimum Lift in a Mach 5 Flow," AIAA 2006-1172, January 2006, presented at the 44th AIAA Aerospace Sciences Meeting and Exhibit, Reno, NV.

[41] Updike, G. A., Shang, J. S., and Gaitonde, D. V., "Hypersonic Separated Flow Control Using Magneto-Aerodynamic Interaction," AIAA 2005-164, January 2005, presented at the 43rd AIAA Aerospace Sciences Meeting and Exhibit, Reno, NV.

[42] Kimmel, R. L., Hayes, J. R., Crafton., J. W., Fonov, S. D., Menart, J., and Shang, J., "Surface Discharge for High-Speed Boundary Layer Control," AIAA 2006-710, January 2006, presented at the 44th AIAA Aerospace Sciences Meeting and Exhibit, Reno, NV.

[43] Bityurin, V. A., Vatazhin, A. B., and Gus'kov, O. V., "Hypersonic Flow Past the Spherical Nose of a Body in the Presence of a Magnetic Field," Fluid Dynamics, Vol. 39, No. 4, July 2004, pp. 657-666.

[44] Miles, R. B., Macheret, S. O., Shneider, M. N., Steeves, C., Murray, R. C., Smith, T., and Zaidi, S. H., "Plasma-Enhanced Hypersonic Performance Enabled by MHD Power Extraction," AIAA 2005-561, January 2005, presented at the 43rd AIAA Aerospace Sciences Meeting and Exhibit, Reno, NV.

[45] Katsurayama, H., Kawamura, M., Matsuda, A., and T., A., "Kinetic and Continuum Simulations of Electromagnetic Control of a Simulated Reentry 
Flow," Journal of Spacecraft and Rockets, Vol. 45, No. 2, March-April 2008, pp. 248-254.

[46] Kim, M., Keidar, M., and Boyd, I. D., "Analysis of an Electromagnetic Mitigation Scheme for Reentry Telemetry Through Plasma," Journal of Spacecraft and Rockets, Vol. 45, No. 6, November-December 2008, pp. 1223-1229.

[47] Macheret, S. O., Shneider, M. N., and Candler, G. V., "Modeling of MHD Power Generation on Board Reentry Vehicles," AIAA 2004-1024, January 2004, presented at the 42nd AIAA Aerospace Sciences Meeting and Exhibit, Reno, NV.

[48] Wan, T., Suzuki, R., Candler, G., Macheret, S., and Schneider, M., "Three Dimensional Simulation of Electric Field and MHD Power Generation During Re-Entry," AIAA 2005-5045, June 2005, presented at the 36th AIAA Plasmadynamics and Lasers Conference, Toronto, Canada.

[49] Fujino, T., Yoshino, T., and Ishikawa, M., "Prediction of Generator Performance and Aerodynamic Heating of Reentry Vehicle Equipped with On-board Surface Hall Type MHD Generator," AIAA 2008-4225, June 2008, presented at the 39th Plasmadynamics and Lasers Conference, Seattle, WA.

[50] Kim, M., Electromagnetic Manipulation of the Plasma Layer for Re-Entry Blackout Mitigation, 2009, PhD thesis, Department of Aerospace Engineering, University of Michigan.

[51] Barmin, A. A., Kulikovskiy, A. G., and Pogorelov, N. V., "Shock-Capturing Approach and Nonevolutionary Solutions in Magnetohydrodynamics," Journal of Computational Physics, Vol. 126, No. 1, June 1996, pp. 77-90.

[52] MacCormack, R. W., "Numerical Simulation of Aerodynamic Flow Including Induced Magnetic and Electric Fields," AIAA 2008-4010, June 2008, presented at the 39th Plasmadynamics and Lasers Conference, Seattle, WA.

[53] D'Ambrosio, D. and Giordano, D., "Two-Dimensional Numerical Methods in Electromagnetic Hypersonics Including Fully Coupled Maxwell Equations," AIAA 2008-4013, June 2008, presented at the 39th Plasmadynamics and Lasers Conference, Seattle, WA.

[54] Tannehill, J. C., Anderson, D. A., and Pletcher, R. H., Computational Fluid Mechanics and Heat Transfer, 2nd ed., 1997, Hemisphere Publishing Corporation.

[55] Moore, G. E., "Progress in Digital Integrated Electronics," Vol. 21, December 1975, pp. 11-13, presented at the 21st International Electron Devices Meeting, Washington, DC. 
[56] Scalabrin, L. C. and Boyd, I. D., "Development of an Unstructured NavierStokes Solver For Hypersonic Nonequilibrium Aerothermodunamics," AIAA 2005-5203, June 2005, presented at the 36th AIAA Thermophysics Conference, Toronto, Canada.

[57] Scalabrin, L. C. and Boyd, I. D., "Numerical Simulation of Weakly Ionized Hypersonic Flow for Reentry Configurations," AIAA 2006-3773, June 2006, presented at the 9th AIAA/ASME Joint Thermophysics and Heat Transfer Conference, San Francisco, CA.

[58] Scalabrin, L. C. and Boyd, I. D., "Numerical Simulations of the FIRE-II Convective and Radiative Heating Rates," AIAA 2007-4044, June 2007, presented at the 39th AIAA Thermophysics Conference, Miami, FL.

[59] MacCormack, R. W. and Candler, G. V., "The solution of the NavierStokes Equations Using Gauss-Seidel Line Relaxation." Computers and Fluids, Vol. 17, No. 1, January 1989, pp. 135-150.

[60] Holman, T. D. and Boyd, I. D., "Numerical Investigation of the Effects of Continuum Breakdown on Hypersonic Vehicle Surface Properties," AIAA 20083928, June 2008, presented at the 40th AIAA Thermophysics Conference, Seattle, WA.

[61] Holman, T. D. and Boyd, I. D., "Effects of Reacting Flow on Continuum Breakdown in Hypersonic Aerothermodynamics," AIAA 2009-4307, June 2009, presented at the 41st AIAA Thermophysics Conference, San Antonio, TX.

[62] Alkandry, H., Boyd, I. D., Reed, E. M., and McDaniel, J. C., "Numerical Study of Hypersonic Wind Tunnel Experiments for Mars Entry Aeroshells," AIAA 2009-3918, June 2009, presented at the 41st AIAA Thermophysics Conference, San Antonio, TX.

[63] Porwitzky, A. J., An End-To-End Model of an Electrothermal Chemical Gun, 2008, PhD thesis, Department of Aerospace Engineering, University of Michigan.

[64] Wright, M. J., A Family of Data-Parallel Relaxation Methods for the NavierStokes Equations, 1997, PhD thesis, Department of Aerospace Engineering and Mechanics, University of Minnesota.

[65] White, F. M., Viscous Fluid Flow, 3rd ed., 2006, McGraw-Hill.

[66] Wilke, C. R., "A Viscosity Equation for Gas Mixtures," Journal of Chemical Physics, Vol. 18, No. 4, April 1950, pp. 517-519.

[67] Scalabrin, L. C., Numerical Simulation of Weakly Ionized Hypersonic Flow Over Reentry Capsules, 2007, PhD thesis, Department of Aerospace Engineering, University of Michigan. 
[68] Karypis, G. and Kumar, V., "METIS: A Software Package for Partitioning Unstructured Graphs, Partitioning Meshes, and Computing Fill-Reducing Orderings of Sparse Matrices," University of Minnesota, MN, 1998.

[69] Park, C., Nonequilibrium Hypersonic Aerothermodynamics, John Wiley \& Sons, Inc., 1990.

[70] Kimmel, R., Klein, M., and Schwoerke, S., "Three-Dimensional Hypersonic Laminar Boundary-Layer Computations for Transition Experiment Design," AIAA Journal, Vol. 34, No. 4, July-August 1997, pp. 409-415.

[71] Kimmel, R. L., Poggie, J., and Schwoerke, S. N., "Laminar-Turbulent Transition in a Mach 8 Elliptic Cone Flow," AIAA Journal, Vol. 37, No. 9, September 1999, pp. 1080-1087.

[72] Candler, G. V., Barnhardt, M. D., Drayna, T. W., Nompelis, I., Peterson, D. M., and Subbareddy, P., "Unstructured Grid Approaches for Accurate Aeroheating Simulations," AIAA 2007-3959, June 2007, presented at the 18th AIAA Computational Fluid Dynamics Conference, Miami, FL.

[73] Gosse, R. and Kimmel, R., "CFD Study of Three-Dimensional Hypersonic Laminar Boundary Layer Transition on a Mach 8 Elliptic Cone," AIAA 20094053, June 2009, presented at the 39th AIAA Fluid Dynamics Conference, San Antonio, TX.

[74] Nowlan, D., Burke, A., and Bird, K., "Pressure and Heat Transfer Distribution on ASD Elliptic Cone (W3) and ASD Sortie (W4) in the CAL 48-Inch Hypersonic Shock Tunnel," Tech. Rep. AM-1800-Y-2, Cornell Aeronautical Laboratory, Inc., Buffalo, NY, December 1963, cleared for public release, ASC 942649.

[75] Atkinson, M., Poggie, J., and Camberos, J. A., "Numerical Investigation of a Blunt-Nosed Elliptical Cone at Hight Angle of Attack in Nonequilibrium Flow," AIAA 2009-4052, June 2009, presented at the 39th AIAA Fluid Dynamics Conference, San Antonio, TX.

[76] Nompelis, I., Computational Study of Hypersonic Double-Cone Experiments for Code Validation, 2004, PhD thesis, Department of Aerospace Engineering and Mechanics, University of Minnesota.

[77] Holden, M. S., Wadhams, T. P., Candler, G. V., and Harvey, J. K., "Experimental Studies of Laminar Separated Flows Induced by Shock Wave/Boundary Layer and Shock/Shock Interaction in Hypersonic Flows for CFD Validation," AIAA 2000-930, January 2000, presented at the 38th AIAA Aerospace Sciences Meeting and Exhibit, Reno, NV.

[78] Tecplot 360 User's Manual, 2008, Tecplot, Inc., 3535 Factoria Blvd S.E. Suite 550, Bellevue, WA 98006. 
[79] Poggie, J., "Plasma-Based Hypersonic Flow Control," AIAA 2006-3567, June 2006, presented at the 37th AIAA Plasmadynamics and Lasers Conference, San Francisco, CA.

[80] Gambit 2.2 User's Guide, 2004, ANSYS, Inc., Southpointe, 275 Technology Drive, Canonsburg, PA 15317.

[81] Young, V., Soaring Over the Crowd, 2009, United States Air Force F-22 Raptor [Online image] Available at http://www.af.mil/shared/media/photodb/photos/090426-F-9497Y-902.jpg [accessed 09 September 2009].

[82] Walker, S. H. and Rodgers, F., "Falcon Hypersonic Technology Overview," AIAA 2005-3253, May 2005, presented at the 13th AIAA/CIRA International Space Planes and Hypersonics Systems and Technologies Conference, Capua, Italy.

[83] Kimmel, R. L., Hayes, J. R., Menart, J. A., and Shang, J., "Effect of Surface Plasma Discharges on Boundary Layers at Mach 5," AIAA 2004-509, January 2004, preseneted at the 42nd AIAA Aerospace Sciences Meeting and Exhibit, Reno, NV.

[84] Velocci, A. L. J., "News Breaks: A General Atomics-led Team," Aviation Week and Space Technology, March 2007, pp. 36.

[85] "U.S. Standard Atmosphere, 1962," Tech. rep., Superintendent of Documents, U.S. Government Printing Office, Washington, D.C., 1962.

[86] Bisek, N. J., Boyd, I. D., and Poggie, J., "Numerical Study of Plasma-Assisted Aerodynamic Control for Hypersonic Vehicles," Journal of Spacecraft and Rockets, Vol. 46, No. 3, May-June 2009, pp. 568-576.

[87] Griffiths, D. J., Introductions to Electrodynamics, 3rd ed., Prentice Hall, 1999.

[88] Choi, J. H., Han, M. H., and Baik, H. K., "Experimental Analysis of Reduced Electric Field During Time-Variable Pulsed Dielectric Barrier Discharge," Japanese Journal of Applied Physics, Vol. 47, No. 2, February 2008, pp. 10711075 .

[89] Datskos, P. G., Christophorou, L. G., and Carter, J. G., "Temperature dependence of Electron Attachment and Detachment in $\mathrm{SF}_{6}$ amnd $\mathrm{c}_{-} \mathrm{C}_{4} \mathrm{~F}_{6}$," The Journal of Chemical Physics, Vol. 99, No. 11, December 1993, pp. 8607-8616.

[90] Belevtsev, A. A., Firsov, K. N., Yu Kazantsev, S., and Kononov, I. G., "On the Temperature Dependence of the Critical Reduced Electric Field in $\mathrm{SF}_{6}$ and Mixtures of $\mathrm{SF}_{6}$ with $\mathrm{C}_{2} \mathrm{H}_{6}$," Journal of Physics D: Applied Physics, Vol. 40, No. 8, April 2007, pp. 1368-1375.

[91] Shercliff, J., A Textbook of Magnetohydrodynamics, 1965, Pergamon Press. 
[92] Gaitonde, D. V. and Poggie, J., "Elements of a Numerical Procedure for 3-D MGD Flow Control Analysis," AIAA 2002-198, January 2002, presented at the 40th AIAA Aerospace Sciences Metting and Exhibit, Reno, NV.

[93] Sutton, G. W. and Sherman, A., Engineering Magnetohydrodynamics, McGraw-Hill, 1965.

[94] Stewart, J., Calculus Early Transcendentals, 4th ed., Brooks/Cole Publishing Company, 1999.

[95] Hoffman, K. A. and Chiang, S. T., Computational Fluid Dynamics - Vol. 1, 4th ed., 2000, Engineering Education System.

[96] Mason, W. H. and Lee, J., "Aerodynamically Blunt and Sharp Bodies," AIAA 1992-2727, June 1992, presented at the 10th AIAA Applied Aerodynamics Conference, Palo Alto, CA.

[97] Gaitonde, D. V., "A High-Order Implicit Procedure for the 3-D Electric Field in Complex Magnetogasdynamic Simulations," Computers and Fluids, Vol. 33, No. 3, March 2004, pp. 345-374.

[98] Oliver, D. A. and Mitchner, M., "Nonuniform Electrical Conduction in MHD Channel," AIAA Journal, Vol. 5, No. 8, August 1967, pp. 1424-1432.

[99] Lieberman, M. A. and Lichtenberg, A. J., Principles of Plasma Discharges and Materials Processing, 2nd ed., John Wiley \& Sons, Inc., 2005.

[100] Weng, Y. and Kushner, M. J., "Method for Including Electron-Electron Collisions in Monte Carlo Simulations of Electron Swarms in Partially Ionized Gases," Physical Review A, Vol. 42, No. 10, November 1990, pp. 6192-6200.

[101] Rockwood, S. D., "Elastic and Inelastic Cross Sections for Electron-Hg Scattering from Hg Transport Data," Physical Review A, Vol. 8, No. 5, November 1973, pp. 2348-2358.

[102] Rockwood, S. D., "Effect of Electron-Electron and Electron-Ion Collisions in $\mathrm{Hg}, \mathrm{CO}_{2} / \mathrm{N}_{2} / \mathrm{He}$, and $\mathrm{CO} / \mathrm{N}_{2}$ discharges," Journal of Applied Physics, Vol. 45, No. 12, December 1974, pp. 5229-5234.

[103] Kushner, M. J., December 2008, private communication.

[104] Dorai, R. and Kushner, M. J., "A Model for Plasma Modification of Polypropylene using Atmospheric Pressure Discharges," Journal of Physics D: Applied Physics, Vol. 36, No. 6, March 2003, pp. 666-685.

[105] Stafford, D. S. and Kushner, M. J., " $\mathrm{O}_{2}\left({ }^{1} \Delta\right)$ Production in $\mathrm{He} / \mathrm{O}_{2}$ mixtures in Flowing Low Pressure Plasmas," Journal of Applied Physics, Vol. 96, No. 5, September 2004, pp. 245-2465. 
[106] Cambel, A. B., Plasma Physics and Magnetofluid-Mechanics, McGraw-Hill, 1963.

[107] Holt, E. H. and Haskell, R. E., Foundations of Plasma Dynamics, The Macmillian Company, 1965.

[108] Bird, G. A., Molecular Gas Dynamics and the Direct Simulation of Gas Flows, Clarendon Press, Oxford, 1994.

[109] Bird, G. A., "Monte-Carlo Simulation in an Engineering Context," Rarefied Gas Dynamics, 1981, edited by S. S. Fisher, Vol. 74 of Progress of Astronautics and Aeronautics, (AIAA, New York, 1981), pp. 239-255.

[110] Poggie, J. and Gaitonde, D. V., "Computational Studies of Magnetic Control in Hypersonic Flow," AIAA 2001-196, January 2001, presented at the 39th Aerospace Sciences Meeting and Exhibit, Reno, NV.

[111] Otsu, H., Abe, T., and Konigorski, D., "Influence of the Hall Effect on the Electrodynamic Heat Shield System for Reentry Vehicles," AIAA 2005-5049, June 2005, presented at the 36th AIAA Plasmadynamics and Lasers Conference, Toronto, Canada.

[112] Otsu, H., Matsuda, A., Abe, T., and Konigorski, D., "Numerical Validation of the Magnetic Flow Control for Reentry Vehicles," AIAA 2006-3236, June 2006, presented at the 37th AIAA Plasmadynamics and Lasers Conference, San Francisco, CA.

[113] Asinovsky, E. I., Kirillin, A. V., Pakhomov, E. P., and Shabashov, V. I., "Experimental Investigation of Transport Properties of Low-Temperature Plasma by Means of Electric Arc," Proceedings of the IEEE, Vol. 59, No. 4, April 1971, pp. 592-601.

[114] Raizer, Y. P., Gas Discharge Physics, Springer-Verlag, 1991.

[115] Godin, D. and Trépanier, J. Y., "A Robust and Efficient Method for the Computation of Equilbrium Composition in Gaseous Mixtures," Plasma Chemistry and Plasma Processing, Vol. 24, No. 3, September 2004, pp. 447-473.

[116] Lamb, L. and Lin, S.-C., "Electrical Conductivity of Thermally Ionized Air Produced in a Shock Tube," Journal of Applied Physics, Vol. 28, No. 7, July 1957, pp. 754-759.

[117] Audet, C., Dennis Jr., J. E., Moore, D. W., Booker, A., and Frank, P. D., "A Surrogate-Model-Based Method For Constrained Optimization," AIAA2000-4891, September 2000, presented at the $8^{\text {th }}$ AIAA/USAF/NASA/ISSMO Symposium on Multidisciplinary Analysis and Optimization, Long Beach, CA. 
[118] Meirlaen, J., Huyghebaert, B., Sforzi, F., Benedetti, L., and Vanrolleghem, P., "Fast, Simultaneous Simulation of the Integrated Urban Wastewater System Using Mechanistic Surrogate Models," Water Science and Technology, Vol. 43, No. 7, 2001, pp. 301-309.

[119] Ong, Y. S., Nair, P. B., and Keae, A. J., "Evolutionary Optimization of Computationally Expensive Problems via Surrogate Modeling," AIAA Journal, Vol. 41, No. 4, April 2003, pp. 687-696.

[120] Cho, Y., Jayaraman, B., Viana, F. A. C., H. R., and Shyy, W., "Surrogate Modeling for Characterizing the Performance of Dielectric Barrier Discharge Plasma Actuator," AIAA 2008-1381, January 2008, presented at the 46th AIAA Aerospace Sciences Meeting and Exhibit, Reno, NV.

[121] Viana, F. A. C. and Goel, T., "SURROGATES Toolbox User's Guide," University of Florida, FL, 2008.

[122] Simpson, T. W., Peplinski, J., Koch, P. N., and Allen, J. K., "On the Use of Statistics in Design and the Implications for Deterministic Computer Experiments," Proceeding from the 1997 ASME Design Engineering Technical Conferences, Bellevue, WA, Vol. 2, September 1997, pp. 1535-1545.

[123] Viana, F. A. C., Haftka, R. T., and Steffen Jr., V., "Multiple Surrogates: How Cross-validation Errors can Help Us to Obtain the Best Predictor," Structural and Multidisciplinary Optimization, Vol. 39, No. 4, October 2009, pp. 439-457.

[124] Mack, Y., CFD-Based Surrogate Modeling of Liquid Rocket Engine Components Via Design Space Refinement and Sensitivity Assessment, 2007, PhD thesis, Mechanical and Aerospace Engineering Department, University of Florida.

[125] Fishman, F., Lothrop, J. W., Patrick, R. M., and Petschek, H. E., "Supersonic Two-Dimensional Magnetohydrodynamic Flow," Standford University Press, Stanford, CA, 1959, pp. 90-119.

[126] Lin, S.-C., Resler, E. L., and Kantrowitz, A., "Electrical Conductivity of Highly Ionized Argon Produced by Shock Waves," Journal of Applied Physics, Vol. 26, No. 1, January 1955, pp. 95-109.

[127] Viana, F. A. C. and Haftka, R. T., "Using Multiple Surrogates For Minimization Of The RMS Error In Meta-Modeling," IDETC/CIE 2008-49240, August 2008, Proceeding of the ASME 2008 International Design Engineering Technical Conferences and Computers and Information in Engineering Conference IDETC/CIE, New York, NY.

[128] Bermejo, S. and Cabestany, J., "Oriented Principal Component Analysis for Large Margin Classifiers," Neural Networks, Vol. 14, No. 10, December 2001, pp. 1447-1461. 
[129] Makridakis, S., "Accuracy Measures: Theoretical and Practical Concerns," International Journal of Forecasting, Vol. 9, No. 4, December 1993, pp. 527529.

[130] Viana, F. A. C., Picheny, V., and T., H. R., "Conservative Prediction Via Safety Margin: Design Through Crossvalidation and Benefits of Multiple Surrogates," IDETC/CIE 2009-87053, August 2009, Proceeding of the ASME 2008 International Design Engineering Technical Conferences and Computers and Information in Engineering Conference - IDETC/CIE, New York, NY.

[131] Bisek, N. J., Boyd, I. D., and Poggie, J., "Numerical Study of Plasma-Assisted Aerodynamic Control for Hypersonic Vehicles," AIAA 2008-4226, June 2008, presented at the 39th AIAA Plasmadynamics and Lasers Conference, Seattle, WA.

[132] Bisek, N. J., Boyd, I. D., and Poggie, J., "Numerical Study of Energy Deposition Requirements for Aerodynamic Control of Hypersonic Vehicles," AIAA 2008-1109, January 2008, presented at the 46th AIAA Aerospace Sciences Meeting and Exhibit, Reno, NV.

[133] Qi, L., "Extrema of a Real Polynomial," Journal of Global Optimization, Vol. 30, No. 4, December 2004, pp. 405-433.

[134] Shang, J. S. and Surzhikov, S. T., "Magneto-Fluid-Dynamics Interaction for Hypersonic Flow Control," AIAA 2004-508, January 2004, presented at the 42nd Aerospace Sciences Meeting and Exhibit, Reno, NV.

[135] Poggie, J., "Numerical Simulation of Direct Current Glow Discharges for High-Speed Flow Control," Journal of Propulsion and Power, Vol. 24, No. 5, September-October 2004, pp. 916-922.

[136] Schwartzentruber, T. E., Scalabrin, L. C., and Boyd, I. D., "Hybrid ParticleCoontinuum Simulations of Non-Equilibrium Hypersonic Blunt Body Flow Fields," AIAA 2006-3602, June 2006, presented at the 9th AIAA/ASME Joint Thermophysics and Heat Transfer Conference, San Francisco, CA.

[137] Arave, R. J. and Huseley, O. A., "Aerothermodynamic Properties of High Temperature Argon," Tech. rep., Boeing, Seattle, WA, February 1962, D211238.

[138] Messerle, H. K., Magnetohydrodynamic Electrical Power Generation, 1995, John Wiley \& Sons, Inc.

[139] Bailey, A. B. and Sims, W. H., "Shock Detachment Distance for Blunt Bodies in Argon at Low Reynolds Number," AIAA Journal, Vol. 1, No. 12, December 1963, pp. 2867-2868.

[140] Poggie, J., October 2009, private communication. 
[141] Sofge, E., "World's Most Powerful Rail Gun Delivered to Navy," Popular Mechanics, November 2007, [Online article], [accessed 15 May 2009]. 